\title{
Question Sets and Answers
}

\section{Acute Care Surgery}

\section{Christian de Virgilio and Areg Grigorian}

\section{Questions}

1. A 55-year-old man presents with a mass in the left groin that is intermittently painful. The mass protrudes upon straining and reduces when he is in the supine position. With the patient standing, there is an obvious mass in his left scrotum that protrudes from the internal ring and becomes more prominent when the patient coughs. Elective surgery is recommended. At surgery, the posterior wall of the hernia sac feels very thickened and is consistent with a possible sliding hernia. Which of the following is true regarding this type of hernia?

(A) Every attempt should be made to excise the entire sac

(B) It poses a higher risk of colon injury during repair

(C) It is more common on the right side

(D) It is most often associated with direct inguinal hernias

(E) The hernia sac should be divided at the internal ring

2. A 66-year-old woman presents to her family doctor complaining of a pain in her left groin that has appeared intermittently over the past several months. On physical exam, a soft mass is palpated in her left groin, below the inguinal ligament, and near her femoral pulse. On palpation, the mass is soft and slightly tender and disappears with gentle compression. Which of the following is true regarding these types of hernias?

(A) They are the most common hernia type in women

(B) The risk of strangulation is relatively low

(C) The hernia sac travels lateral to the femoral vein

(D) If discovered incidentally and the patient is asymptomatic, repair is not indicated

(E) It is associated with multigravida
3. A 30-year-old woman is recovering from an open cholecystectomy in the hospital. On the second postoperative day, she begins to complain of cramping abdominal pain without vomiting. She has no past medical or surgical history, and her postoperative course has been unremarkable. She is receiving oral hydrocodone for pain and is on a clear liquid diet. She has a temperature of $99.5^{\circ} \mathrm{F}$, blood pressure is $128 / 84 \mathrm{mmHg}$, and pulse is $82 / \mathrm{min}$. Her physical exam is significant for absent bowel sounds, a mildly distended abdomen with mild diffuse tenderness without rebound or guarding. Which of the following would most benefit her abdominal findings?

(A) Encouraging ambulation

(B) Placement of a nasogastric tube

(C) Neostigmine

(D) Conversion of hydrocodone to a nonsteroidal antiinflammatory drug

(E) Return to the operating room for exploration

4. A Richter's hernia:

(A) Most often contains colon or bladder in the posterior aspect of the sac

(B) Has a low risk of incarceration

(C) Most commonly presents as a small bowel obstruction

(D) Can mislead the clinician as strangulated bowel can easily be missed

(E) Should be manually reduced in the emergency department provided there is no evidence of bowel obstruction

5. A 55-year-old schizophrenic homeless man arrives to the ED with abdominal pain and vomiting. He reports that the abdominal pain started yesterday and has been worsening. He is afebrile, blood pressure is $122 / 86 \mathrm{mmHg}$, and heart rate is $116 / \mathrm{min}$. In the ED he vomits green emesis without blood. His last bowel movement was $48 \mathrm{~h}$ 
ago. Physical examination reveals a large scar in his right upper quadrant. On abdominal examination, the abdomen is distended, with hyperactive bowel sounds, and is tympanic to percussion, with mild diffuse tenderness, and no rebound or guarding. WBC is $9 \times 10^{3} / \mu \mathrm{L}$ (normal $\left.4.1-10.9 \times 10^{3} / \mu \mathrm{L}\right)$. Abdominal series shows dilated loops of bowel with multiple air fluids levels. After fluid resuscitation, what is the most appropriate next step in management?
(A) Nasogastric tube suction
(B) Laparoscopy
(C) Exploratory midline laparotomy
(D) Intravenous erythromycin
(E) CT scan of the abdomen

6. A worried mother presents to you with concerns that her 6-month-old boy has a large protrusion at his belly button that is worse when he cries but reduces when he is sleeping. On exam you palpate a $1 \mathrm{~cm}$ fascial defect at his umbilicus. Which of the following is true about this condition?
(A) Elective repair is recommended
(B) The condition is associated with cardiac anomalies
(C) The size of the defect predicts that it will not likely close on its own
(D) The risk of incarceration is significant
(E) Repair should be delayed until the child is 4 years old

7. One week after open repair of a large right scrotal hernia, a 45-year-old male returns complaining of severe pain in his right testicle. On physical exam, the testicle appears to be slightly swollen and very tender to palpation. Doppler study demonstrates no flow within the right testicle with normal flow in the left. Which of the following is true about this condition?

(A) It is most commonly due to thrombosis of the pampiniform plexus

(B) Urgent exploration of the right testicle is recommended
(C) It is most likely due to transection of the testicular artery

(D) It most likely represents testicular torsion

(E) The testicle will likely remain permanently enlarged

8. Following open inguinal hernia repair, a 50-year-old male complains of numbness and burning pain on the scrotum. This most likely represents injury to:

(A) The genital branch of the genitofemoral nerve

(B) The femoral branch of the genitofemoral nerve

(C) The ilioinguinal nerve

(D) The lateral femoral cutaneous nerve

(E) The iliohypogastric nerve

9. A 65-year-old male presents to the ED with nausea, vomiting, and severe abdominal pain. Past history is significant for prior sigmoid colectomy for diverticulitis 10 years ago. On physical exam, his temperature is $100.9^{\circ} \mathrm{F}$, blood pressure is $110 / 80 \mathrm{mmHg}$, and heart rate is $110 / \mathrm{min}$. His abdomen has a well-healed midline scar and is distended. Bowel sounds are hyperactive with occasional rushes and tinkles. He has marked right upper quadrant tenderness to palpation with guarding. The rest of the abdominal exam is unremarkable. Abdominal series demonstrates one loop of markedly distended small bowel in the right upper quadrant with an air fluid level. No gas is seen in the colon or rectum. Laboratory values demonstrate a WBC count of $18 \times 10^{3} / \mu \mathrm{L}$ (normal $4.1-10.9 \times 10^{3} / \mu \mathrm{L}$ ) with $15 \%$ bands and a serum lactate of $5 \mathrm{mmol} / \mathrm{L}$ (normal $0.5-1.6 \mathrm{mmol} / \mathrm{L}$ ), BUN $30 \mathrm{mg} / \mathrm{dL}(7-21 \mathrm{mg} / \mathrm{dL})$, and creatinine $1.2 \mathrm{mg} / \mathrm{dL}$ (0.5-1.4 mg/dL). Amylase, lipase, and liver chemistries are normal. NG tube and IV fluids are given. What is the next step in the management?

(A) Exploratory laparotomy

(B) Admit for close observation

(C) Upper GI with small bowel follow through with barium

(D) Upper GI with small bowel follow through with Gastrografin

(E) Right upper quadrant ultrasound 


\section{Answers}

\section{Answer B}

Sliding inguinal hernias have a much higher risk of colonic injury during repair than other hernias. This is because the posterior wall of the hernia sac is formed by a retroperitoneal organ (colon or bladder). A clue to the presence of a sliding hernia is the finding of a thickened posterior wall of the hernia sac at surgery, in association with a large indirect hernia (D) that has descended into the scrotum (direct hernias rarely descend into the scrotum). Attempting to completely excise the hernia sac (A) (which is otherwise normally done), or to divide the sac completely at the internal ring (E) (which is again normally recommended), would result in dividing the bowel or bladder. Sliding hernias are more common on the left side (C) (the sigmoid colon is less fixed and more likely to slide down than the right colon). A sliding hernia is an indirect inguinal hernia (D).

\section{Answer $\mathrm{E}$}

Multigravida causes stretching of the abdominal musculature and increases the risk of femoral hernia. Femoral hernias occur in the femoral canal, inferior to the inguinal ligament traversing the empty space medial $(C)$ to the femoral vein (recall the mnemonic "NAVEL" \{from lateral to medial: femoral nerve, artery, vein, empty space, lymphatic \}). The most common type of hernia in women, and in men, is an indirect inguinal hernia (A). Although femoral hernias appear infrequently (10\% of all hernias), they occur more commonly in females and have the highest risk of strangulation (B). Because of the high risk of strangulation, surgical repair of a femoral hernia is indicated (D) once diagnosed, regardless of whether the patient is having symptoms.

\section{Answer D}

Always consider a nonmechanical postoperative ileus in patients that have had a recent surgery. This occurs in up to $50 \%$ of patients that have undergone abdominal surgery. Although the exact cause has not been elucidated, it most likely involves impaired peristalsis of intestinal contents. Inflammatory mediators (e.g., recent surgery) and opioid analgesics are thought to contribute to the development of postoperative ileus. Initial management should begin with changing pain medication to a non-opiate analgesic. Encouraging ambulation (A) should also be done for all postoperative patients, but is not as imperative as discontinuing opiates. If the patient's postoperative ileus continues with worsening symptoms (e.g., emesis), bowel decompression including a NGT (B) can be considered. Returning to the OR for exploration $(\mathrm{E})$ is inappropriate for postoperative ileus. Neostigmine is used in patients with pseudo-obstruction (Ogilvie's syndrome).

\section{Answer D}

With a Richter's hernia, only one wall of the bowel protrudes into the hernia sac (A). That segment of bowel is prone to incarceration and strangulation but does so without associated symptoms, signs, or radiologic evidence of SBO (C). Therefore, it may easily mislead clinicians into thinking that the hernia is not incarcerated (B). Manual reduction of hernias (including Richter's) should not be attempted if strangulation is suspected as dead bowel will be reduced into the peritoneum. Strangulation should be suspected in the presence of fever, leukocytosis, acidosis, severe pain, or marked erythema overlying the skin of the hernia. It is often difficult to palpate a Richter's hernia, and it should be reduced in the operating room $(\mathrm{E})$.

\section{Answer A}

This patient has evidence (on history, physical, and radiologic imaging) of a small bowel obstruction (SBO) that is most likely secondary to adhesions from prior surgery (scar in RUQ). SBO from adhesions can present many years after surgery. The initial management of SBO includes placing the patient NPO, aggressive intravenous fluid resuscitation (the patient is tachycardic and likely very dehydrated), and NG tube placement. Aside from the salutatory effect of $\mathrm{NG}$ decompression on the distended bowel, patients with SBO are at risk of aspiration. Once the patient has been adequately resuscitated, CT scan $(\mathrm{E})$ with oral contrast is recommended as it is useful in confirming the diagnosis of SBO, determining if the SBO is partial or complete, and ruling out other diagnosis. Most patients with SBO due to adhesions improve with these maneuvers, and do not require surgery. Operative management $(\mathrm{C})$ with laparotomy and lysis of adhesions should be considered in the following conditions: if the patient demonstrates evidence of clinical deterioration as manifest by increasing pain, tenderness, fever, leukocytosis, or acidosis. Operative management can be achieved either via open laparotomy or laparoscopy (B). Evidence of a complete SBO is a relative indication for surgery, but recent studies suggest that some of these patients resolve with nonoperative management as well. Intravenous erythromycin acts as a prokinetic agent and has some utility for gastroparesis, but not for a SBO (D).

\section{Answer E}

This patient has an umbilical hernia, which is a common finding in newborns. It is recommended that repair be delayed (A) until after the child is 4 years old, unless the defect is larger than $2 \mathrm{~cm}$, the defect is growing, or there is evidence of strangulation. Umbilical hernias are not associated with the VACTERL (vertebral, anal, cardiac, tracheoesophageal fistula, renal, limb) complex of anomalies (B). Defects smaller than $2 \mathrm{~cm}$ will likely close spontaneously (C). It is very rare for umbilical hernias in children to incarcerate (D). 


\section{Answer A}

This patient likely has ischemic orchitis secondary to damage to or thrombosis of the pampiniform plexus. This is most likely to occur in patients with large or densely adhesed hernia sacs. The condition is usually self-limited (E), so urgent exploration (B) is not indicated. Ischemic orchitis is more commonly caused by injury to the pampiniform plexus than to the testicular artery (C). Testicular torsion (D) is less likely than a vascular injury in this case, although both would present with acute testicular pain and decreased or absent Doppler signal.

\section{Answer A}

The genital branch of the genitofemoral nerve provides sensation to the scrotum and the cremaster reflex. The femoral branch of the genitofemoral nerve (B) provides sensation to the proximal medial thigh. The ilioinguinal nerve (C) provides sensation to the lower abdomen and medial thigh. The lateral femoral cutaneous nerve (D) provides sensation to the lateral thigh as low as the knee. The iliohypogastric nerve (E) supplies the gluteal region.

\section{Answer A}

This patient has a SBO with evidence of ischemic or gangrenous bowel most likely secondary to adhesions from past surgery (e.g., sigmoidectomy). Necrotic bowel generally does not occur in association with a SBO unless there is a closed-loop obstruction. A closed-loop obstruction is a particularly dangerous form of bowel obstruction in which a segment of intestine is obstructed both proximally and distally. Gas and fluid accumulate within this segment of bowel, and cannot escape. This progresses rapidly to strangulation with risk of ischemia, gangrene, and subsequent perforation. Clues to ischemic bowel include the presence of acidosis, fever, leukocytosis, and severe localized pain (unusual for SBO). As such the patient will need exploratory laparotomy, and any bowel that is obviously nonviable needs to be resected. Most patients with SBO (without necrotic bowel) due to adhesions improve with conservative management, and do not require surgery. Observation is not appropriate for this patient (B). Upper GI studies (C-D) would not be indicated since this patient has strong evidence of necrotic bowel and requires urgent surgical intervention. RUQ ultrasound (E) is appropriate in the workup for cholelithiasis. 


\section{Breast Cancer}

\section{Areg Grigorian and Christian de Virgilio}

\section{Questions}

1. A 30-year-old female presents with bloody discharge from her left breast that she has noticed intermittently for the past month. She denies any palpable breast mass, weight loss, fevers, or night sweats. She has no medical history or family history of breast cancer. The skin around the breast and areola are normal with no rashes or lesions. No breast mass is palpable, and there is no axillary lymphadenopathy. Ultrasound did not reveal any masses. What is the most likely diagnosis?
(A) Fibrocystic changes
(B) Intraductal papilloma
(C) Ductal carcinoma in situ (DCIS)
(D) Paget's disease of the breast
(E) Infiltrating ductal carcinoma

2. A 61-year-old female presents with swelling and redness of her entire left breast that has persisted for 4 weeks. On physical exam her temperature is $98.7^{\circ} \mathrm{F}$, pulse is $82 / \mathrm{min}$, blood pressure is $136 / 78 \mathrm{mmHg}$, and respirations are 16/min. Her left breast appears larger than her right one. The entire breast is warm, and the skin is edematous. No breast masses are palpable. There is no nipple discharge or rashes. There are several palpable enlarged lymph nodes in her left axilla. Ultrasound and mammography show thickening of the skin but otherwise no masses. Which of the following is the best option for further management?
(A) Punch biopsy of skin
(B) Oral antibiotics
(C) Intravenous antibiotics
(D) Nonsteroidal anti-inflammatory drugs
(E) Incision and drainage

3. A 36-year-old woman is evaluated for a lump in her right breast that she noticed 5 months ago. She denies any nipple discharge, nipple retraction, or skin changes. She has no family history of breast cancer. On physical exam, the breasts appear normal. Palpation reveals a $1 \mathrm{~cm}$ dominant lump in the left upper quadrant that does not appear to be fixed to the surrounding structures. The patient has no other dominant masses in either breast. There is no axillary lymphadenopathy. Mammogram is negative. What is the next step in the management?

(A) Ultrasound-guided core needle biopsy

(B) Fine-needle aspiration

(C) MRI
(D) Follow-up clinical breast exam in 3 months

(E) Genetic testing

4. A 31-year-old breastfeeding female comes to the doctor for localized swelling, redness, and pain of the left breast. She also reports muscle aches and fatigue. On physical exam her temperature is $101.1^{\circ} \mathrm{F}$, pulse is $82 /$ min, blood pressure is $126 / 68 \mathrm{mmHg}$, and respirations are $16 / \mathrm{min}$. Physical exam reveals a localized area of erythema and warmth in the left breast with no palpable masses. There is no axillary lymphadenopathy. What is the most likely next course of action?
(A) Biopsy
(B) Antibiotic treatment and continue breast feeding
(C) Antibiotic treatment and encourage bottle-feeding only
(D) Diagnostic mammography
(E) Incision and drainage

5. A 17-year-old female presents with breast pain that she noticed for several months. She states that she feels multiple breast masses in both breasts. She denies any weight loss, fevers, or night sweats. She has no medical history or family history of breast cancer. Physical examination reveals that her heart has a regular rate and rhythm. The skin around the breast and areola are normal with no rashes or lesions. No solitary breast masses are palpable, but both breasts are lumpy and painful to palpation, most notably in the upper outer quadrants. There is no axillary lymphadenopathy. What is the most appropriate next step in management?
(A) Diagnostic mammography
(B) Excisional biopsy
(C) Ultrasound-guided core needle biopsy
(D) Reassurance and reexamine in 1 month
(E) Fine-needle aspiration (FNA)

6. A 71-year-old woman is evaluated for a lump in her right breast that she noticed 3 weeks ago. She denies any nipple discharge, nipple retraction, or skin changes. She has a sister who was diagnosed with breast cancer at the age of 57. She had menarche at the age of 9 and menopause at the age of 56. She had two children, one at the age of 39 and the other at the age of 41 . On physical exam, the breasts are normal on inspection. Palpation reveals a $1.5 \mathrm{~cm}$ dominant lump that does not appear to be fixed to the surrounding structures in the left upper, outer quadrant. The patient has no other dominant masses in either breast. There is no axillary lymphadenopathy. What is the biggest risk factor in this patient predisposing her to breast cancer?

(A) Early menarche

(B) Family history of breast cancer 

(C) Older age
(D) Age at first pregnancy
(E) Late menopause

7. A 50-year-old woman comes to clinic to discuss treatment for a new diagnosis of breast cancer. Her annual screening mammogram revealed a $1.3 \mathrm{~cm}$ mass in the right breast. The patient does not have any other breast masses, skin changes, nipple discharge, or axillary adenopathy. Mammography revealed no other suspicious calcifications within the breast. Biopsy of the mass was performed and revealed infiltrating ductal carcinoma. Estrogen receptor, progesterone receptor, and Her2/neu receptor testing were negative. Which of the following is the best option for the management of this patient's breast cancer?

(A) Lumpectomy and breast irradiation

(B) Lumpectomy and hormone therapy

(C) Lumpectomy and chemotherapy

(D) Lumpectomy, sentinel node biopsy, and breast irradiation

(E) Lumpectomy, sentinel node biopsy, breast irradiation, and chemotherapy

8. A 65-year-old woman returns to clinic for a 3-month follow-up. Three months ago she developed a pruritic, erythematous, ulcerated rash surrounding the areola of her right breast. She tried hydrocortisone $1 \%$ on the lesion at the recommendation of her primary care physician, but the lesion persisted. She has no history of skin diseases in the family. She takes warfarin for atrial fibrillation. She started a new medication, hydrochlorothiazide, for hypertension about 3 months ago. Otherwise, she is healthy. What is the best next step in the management of this patient?

(A) Punch biopsy of the skin lesion

(B) Change hydrocortisone $1 \%$ to triamcinolone to treat eczema
(C) Treatment with antibiotics

(D) Oral steroid course to treat psoriasis

(E) Increase the dose of hydrocortisone

9. A 57-year-old woman comes to clinic to discuss surgical treatment for a new diagnosis of breast cancer. Her annual screening mammogram revealed a $1.7 \mathrm{~cm}$ mass in the right breast. Biopsy of the mass was performed and revealed infiltrating ductal carcinoma. Estrogen receptor and progesterone receptor testing were negative, while HER-2 receptor testing was positive. In addition to lumpectomy and breast irradiation, the treating doctor decides to add hormonal therapy with trastuzumab to the treating regimen. What study must be done prior to starting trastuzumab?
(A) TSH and free T4
(B) Liver function tests
(C) Echocardiogram
(D) Creatinine clearance
(E) CXR

10. A 45-year-old female undergoes screening mammography which demonstrates an area of suspicious microscopic calcification in her right upper outer breast. Stereotactic-guided biopsy confirms ductal carcinoma in situ (DCIS). Which of the following is true about this condition?

(A) It should be excised to a negative margin

(B) It is considered a marker for malignancy in either breast

(C) The cribriform type has a worse prognosis than the comedo type

(D) It does not occur in men

(E) Radiation therapy is an acceptable alternative to surgical excision 


\section{Answers}

\section{Answer B}

Although bloody nipple discharge should raise concern for cancer, intraductal papilloma is the most common cause of bloody nipple discharge. This is a benign breast tumor arising from the proliferation of mammary duct epithelium that classically occurs in females 20-40 years of age. Treatment includes excision, which is diagnostic as well as curative. Fibrocystic changes (A) are a common cause of breast pain in young females. Patients report painful breast tissue before menses with improvement during menstruation. Physical exam reveals fibrotic tissue and cystic, lumpy tissue. It may be associated with bilateral serous discharge. DCIS (C) and infiltrating ductal carcinoma (E) are more common in older women. DCIS most often presents as suspicious calcifications on mammography, and not with bloody nipple discharge. Although breast cancer can present with bloody nipple discharge, it is less common than intraductal papilloma, especially in a young woman. Paget's disease of the breast (D) causes an eczematous lesion on the breast that is associated with an underlying breast carcinoma. Given that this patient's skin exam is normal, this diagnosis is unlikely.

\section{Answer A}

The patient most likely has inflammatory breast carcinoma, an especially aggressive type of breast cancer. Inflammatory breast cancer can be easily confused with mastitis, as there is usually no palpable breast mass and ultrasound and mammography similarly are often negative. As such, it is imperative to perform a biopsy of the skin, which may show cancer cells invading the subdermal lymphatics. Additional workup should include a breast MRI (which is more likely to show the breast cancer in this setting than ultrasound and mammogram), as well as consideration for needle biopsy of the lymph nodes. Antibiotics (B-C) or NSAIDs (D) would be inappropriate. Incision and drainage (E) would be appropriate if there was an indication on physical examination or evidence of a breast abscess on ultrasound. Inflammatory breast carcinoma typically presents as swelling of the breast and with edematous skin due to obstruction of subdermal lymphatics by tumor (termed peau d'orange, meaning orange peel in French). At presentation, positive lymph node involvement is frequent, and approximately one-third of patients have distant metastases. Inflammatory breast carcinoma can present during pregnancy and should be suspected if suspected mastitis does not respond to appropriate antibiotic treatment.

\section{Answer A}

A diagnostic mammogram should be ordered in a woman over the age of 30 who presents with a new breast mass. Mammography helps to look for suspicious calcifications in other areas of the affected breast, characterize the mass, as well as evaluate the contralateral breast. It is important to note that the mammogram may be normal despite the presence of a palpable breast cancer. For this reason, a tissue biopsy is recommended for palpable breast masses regardless of the mammogram results. Tissue sampling is best performed via ultrasound-guided core needle biopsy. Ultrasound also provides more information about the mass (cystic vs. solid). Fine-needle aspiration (B) is rarely used as it relies on cytology rather than histology. MRI (C) is not routinely needed. Follow-up examination in 3 months (D) without a biopsy would be inappropriate. Genetic testing (E) would be indicated if this patient had a strong family history of breast or ovarian cancer, but would not be done until tissue diagnosis of breast cancer is confirmed.

\section{Answer B}

The patient most likely has lactation mastitis. Lactation mastitis is a localized, painful inflammation of the breast accompanied by fever and malaise occurring in breastfeeding women. The diagnosis of mastitis is made clinically based on an erythematous, tender, swollen area of one breast associated with fever in a nursing mother. Other symptoms may include muscle pain (myalgias) and malaise. Transmission occurs via introduction of bacteria in small breaks in the skin caused by the trauma of breastfeeding. Most cases of lactation mastitis are a result of an infection by Staphylococcus aureus. Treatment consists of antibiotics to cover skin flora, symptomatic relief with analgesics including anti-inflammatory agents such as ibuprofen, and cold compresses to reduce local pain and swelling. Patients should be encouraged to continue breastfeeding (C) as this helps relieve any ductal obstruction that might be contributing to the infection. Biopsy (A) would be appropriate if the patient has suspected inflammatory breast carcinoma. Although very rare, inflammatory breast carcinoma can occur during pregnancy. If mastitis fails to resolve after antibiotics, then consideration should be given to performing a biopsy of the skin. Diagnostic mammography (D) would not be indicated at this time. Incision and drainage (E) is appropriate if there was evidence of a localized abscess with fluctuance. Ultrasound can help differentiate mastitis from a breast abscess.

\section{Answer D}

The history and physical exam is most consistent with a diagnosis of fibrocystic changes of the breast, which is considered a normal variant of the breast in adolescents and young adults. Patients will present with painful breast tissue before menses that improves during menstruation. On examination, fibrotic tissue may be palpated and is generally found in the upper outer quadrants of the breast. This patient should be counseled and instructed to look for these changes with a follow-up appointment in a month. Persistent cystic breast lesions can be evaluated and treated with fine-needle 
aspiration (E), although this is not be needed in children and adolescents. Cystic lesions that resolve with aspiration should be reevaluated with ultrasonography 3 months after aspiration (C). Excisional biopsy (B) may be warranted for cystic lesions that do not resolve with aspiration or for suspicious solid lesions. Diagnostic mammography (A) is not indicated for adolescents and should be reserved for females $>30$ years old who present with a breast mass.

\section{Answer $\mathrm{C}$}

The most important risk factors for breast cancer are female gender, increasing age, and a family history of premenopausal breast cancer. A new breast mass in a woman over the age of 50 should be considered cancer until proven otherwise, as it carries the highest relative risk of being cancer. A family history of breast cancer (B) can also significantly increase the risk of breast cancer, particularly if diagnosed in a premenopausal woman. The majority of inherited breast cancers are associated with BRCA1 or BRCA2 gene mutations. Other important risk factors associated with a slightly higher risk of developing breast cancer include early menarche (A), nulliparity or older age at first full-term pregnancy (D), and/or late menopause (E).

\section{Answer $\mathrm{E}$}

This patient is diagnosed with infiltrating ductal carcinoma. Treatment for stage I and II breast cancers includes the option of breast conserving therapy (BCT), which consists of excision of the primary tumor (lumpectomy), sentinel lymph node biopsy (SLNB), followed by radiation therapy to the remaining breast. Studies have shown that breast conserving therapy leads to survival rates that are equivalent to that of mastectomy (though a higher local recurrence rate), while providing a more aesthetically pleasing surgical result. Triple negative breast cancers (ER, PR and Her2/neu receptor) are thought to have a worse prognosis as it is insensitive to some of the best therapies (tamoxifen and aromatase inhibitors for hormone positive, and trastuzumab for Her2/neu positive). As such, chemotherapy is recommended postoperatively.

\section{Answer A}

The presentation is concerning for Paget's disease of the breast. This presents as an eczematous, scaling, and ulcerating lesion around the areola. Paget's disease of the breast is a type of DCIS that extends into the ducts to involve the skin of the nipple. Patients are initially misdiagnosed with a skin condition, including eczema and psoriasis, and receive a variety of ointments that do not resolve the lesion. Paget's disease of the breast is almost always associated with an underlying carcinoma and must be diagnosed via biopsy of the lesion. Trying different regimens of steroids and antibiotics is inappropriate given the high likelihood that she has cancer $(\mathrm{B}-\mathrm{E})$.

\section{Answer C}

Trastuzumab is a monoclonal antibody that blocks the HER-2 receptors. The medication is used in the treatment of HER-2-positive breast cancers to help reduce recurrence and improves survival. Since there is a high risk of cardiomyopathy in patients receiving trastuzumab, it is recommended that all patients receive an echocardiogram prior to initiating therapy with trastuzumab. An alternative is to obtain a MUGA scan (multigated acquisition scan), which is a nuclear study that evaluates ventricular function. Trastuzumab-related cardiotoxicity is most often manifested by an asymptomatic decrease in ejection fraction. The optimal surveillance for trastuzumab-related cardiotoxicity is not well defined. The remaining answer choices are not needed prior to starting trastuzumab (A-B, D-E).

\section{Answer A}

DCIS is characterized by malignant epithelial cells within the mammary ductal system, without invasion into the surrounding stroma. Comedo-type DCIS is typically high grade and associated with a worse prognosis (C). DCIS lesions have a high risk of subsequent invasive carcinoma at the site of the DCIS. As such if left unresected, it will often progress to invasive ductal cancer. Thus the mainstay of DCIS treatment is lumpectomy (excision of entire lesion with negative margins). Lobular carcinoma in situ is considered a marker for malignancy in either breast (B). Breast cancer in males is rare ( $1 \%$ of all breast cancers) with most cases identified as invasive ductal carcinoma. DCIS can occur in men but is even more rare, as DCIS most often presents as abnormal calcifications on mammogram (D). Radiation therapy can be used in combination with surgical excision, but cannot replace it $(\mathrm{E})$. 


\section{Cardiothoracic}

\section{Areg Grigorian, Paul N. Frank, and Christian de Virgilio}

\section{Questions}

1. A 65-year-old male presents with a painful nodule in his wrist that is determined to be a ganglion cyst. Despite attempts at aspiration, it recurs. He is unable to work as a computer programmer, is on disability, and is feeling depressed. He is scheduled for wrist surgery. He reports having been discharged 1 week ago for an episode of chest pain. Troponins were elevated at that time, but there was no elevation of his ST segment. Which of the following is the best recommendation?

(A) Proceed with surgery with intraoperative transesophageal echocardiography

(B) Proceed with surgery but perform under local anesthesia with sedation

(C) Proceed with surgery only if echocardiogram shows normal ejection fraction

(D) Proceed with surgery after aggressive beta blockade to get heart rate into low $60 \mathrm{~s}$

(E) Postpone surgery for at least 4 weeks

2. A 65-year-old male is about to undergo an elective inguinal hernia repair. Which of the following findings on history or physical would portend the highest operative risk?

(A) Systolic, crescendo-decrescendo murmur at the sternal border of the right second intercostal space radiating into neck

(B) A history of myocardial infarction 10 years ago

(C) Insulin-dependent diabetes mellitus with an elevated $\mathrm{HgbA} 1 \mathrm{C}$

(D) Renal insufficiency not yet on dialysis

(E) Smoking

3. A 65-year-old male undergoes a videoscopic right upper lobectomy for squamous cell lung cancer. On postoperative day one, he suddenly develops chest pain and diaphoresis. Blood pressure is $120 / 60 \mathrm{mmHg}$, and heart rate is $80 / \mathrm{min}$. Serial highly sensitive troponin I assays demonstrate levels of $0.4,0.3$, and $0.01 \mathrm{ng} / \mathrm{dl}$. ECG demonstrates nonspecific $\mathrm{T}$ wave changes with no ST segment elevation. Following the administration of oxygen, morphine, aspirin, and a beta-blocker, his symptoms resolve. What is the next step in the management?

(A) Intravenous thrombolytic therapy

(B) Percutaneous coronary intervention without stenting

(C) Percutaneous coronary intervention with stenting

(D) Coronary artery bypass graft (CABG)

(E) Continue medical management and reevaluate as outpatient in 4-6 weeks
4. A 17-year-old African American male presents for a preparticipation physical before track season. A harsh systolic murmur is heard at the second right intercostal space. He denies ever experiencing chest pain, dizziness, or difficulty breathing. Which of the following would be expected on further workup?

(A) $\mathrm{T}$ wave inversion on ECG

(B) Laterally displaced PMI on palpation

(C) Weak femoral pulses compared to brachial pulses

(D) Increased intensity of the murmur with Valsalva maneuver

(E) Increased intensity of the murmur with squatting

5. A 65-year-old woman arrives to the ED complaining of chest pain. Her past medical history includes hypertension, atherosclerosis, and coronary artery disease. She underwent a coronary artery bypass graft (CABG) 3 weeks ago for three-vessel disease. She reports that her chest pain worsens with inspiration and lessens when leaning forward. A friction rub is heard on auscultation. ECG shows global ST elevation. What is the most likely diagnosis?
(A) Myocarditis
(B) Myocardial infarction
(C) Cardiac tamponade
(D) Acute pericarditis
(E) Pulmonary embolism

6. An obese 52-year-old man with a 50-pack-year smoking history and hypertension controlled with chlorthalidone presents to a remote hospital without interventional capabilities with 30 min of crushing chest pain radiating to his left arm and jaw. Troponin and CK-MB levels are elevated, and ECG shows ST segment elevations in leads V1 through V4. He is treated with thrombolytic therapy, and his symptoms resolve. The next morning, the patient is found dead in his bed. Which of the following is the most likely cause of death?
(A) Ventricular free wall rupture
(B) Embolic stroke
(C) Ventricular arrhythmia
(D) Post-MI pericarditis
(E) Overwhelming infection

7. A 65-year-old female has breast cancer and a remote history of congestive heart failure. Her physician is planning to administer a chemotherapeutic agent that has potential for cardiac toxicity. Which of the following is the most accurate test to measure ejection fraction?
(A) Multi Gated Acquisition Scan (MUGA) scan
(B) Echocardiography
(C) Electrocardiogram
(D) Coronary angiography
(E) Exercise stress test 
8. A 76-year-old man is driven to the ED by his wife and is complaining of severe chest pain that started $30 \mathrm{~min}$ ago. $\mathrm{He}$ denies abdominal or extremity pain. Pulses in arms and legs are 2+. His kidney function is normal. CT scan shows an aortic dissection. Which of the following findings on CT scan would most strongly indicate the need for urgent surgery?
(A) Dissection of entire descending thoracic aorta
(B) Involvement of common iliac arteries
(C) Involvement of renal arteries
(D) Extension into mesenteric vessels
(E) Involvement of origin of innominate artery

9. A 65-year-old female is diagnosed with aortic dissection beginning $2 \mathrm{~cm}$ distal to the left subclavian artery and extending distally. Her blood pressure is $180 / 70 \mathrm{mmHg}$, and her heart rate is $88 / \mathrm{min}$. Peripheral pulses are all $2+$, and her abdomen is soft and non-tender. What is the next best step in treatment?
(A) Surgical repair
(B) Aggressive IV fluids
(C) Labetalol drip
(D) Endovascular repair
(E) Nicardipine drip

10. A 65-year-old man is rushed to the ED by ambulance after he suddenly lost strength and sensation in his left leg and arm. He was hospitalized 2 months ago with a NSTEMI. He is compliant with all of his medications and had been recovering well until the present episode. ECG shows normal sinus rhythm without evidence of ischemia. Chest X-ray is unremarkable. Carotid ultrasounds show $<30 \%$ stenosis bilaterally. What is the most likely etiology of the patient's present symptoms?
(A) Ventricular thromboembolism
(B) Septic embolism to the brain
(C) Type A dissection involving the right carotid artery
(D) Thromboembolism from the left atrial appendage
(E) Paradoxical venous thromboembolism

11. A 66-year-old man is recovering in the ICU after receiving a CABG for coronary artery disease. On the fourth postoperative day, he complains of chest pain. He is sweating, anxious, short of breath, and nauseated. ECG shows evidence of right-sided MI. His blood pressure is $98 / 65 \mathrm{mmHg}$. What is the next best step in management?
(A) Administer $1 \mathrm{~L}$ of normal saline
(B) Nitroglycerin
(C) Nitroprusside
(D) Nifedipine
(E) Lisinopril

12. A 63-year-old woman with diabetes is recovering in the ICU after receiving a CABG for coronary artery disease. On the sixth postoperative day, she starts complaining of chest pain. Her temperature is $101.4^{\circ} \mathrm{F}$, blood pressure is $108 / 72 \mathrm{mmHg}$, and pulse is $125 / \mathrm{min}$. On physical exam, there is drainage from her sternal wound, and there is a crunching sound heard with a stethoscope over the precordium during systole. The sternum feels somewhat unstable to palpation. Her laboratory examination is significant for an elevated white blood count $\left(16.7 \times 10^{3} / \mu \mathrm{L}\right)$. What is the most likely diagnosis?
(A) Acute pericarditis
(B) Postoperative MI
(C) Empyema
(D) Acute mediastinitis
(E) Pneumonia

13. A 75-year-old male with severe aortic stenosis has a routine check-up at his primary care doctor. Which of the following symptoms portends the worst prognosis?
(A) Exertional chest pain
(B) Swollen legs
(C) Fainting spells
(D) Mid-systolic murmur heard loudest at the upper right sternal border
(E) Small head nodding movements at each heartbeat

14. Which of the following is the most important risk factor for aortic dissection?

(A) History of coronary artery bypass grafting $(\mathrm{CABG})$

(B) Giant cell arteritis

(C) Pregnancy

(D) Hypertension

(E) Bicuspid aortic valve

15. A patient is diagnosed with type A aortic dissection, and there is concern for cardiac tamponade. Which of the following findings would be the MOST consistent with cardiac tamponade?
(A) Pulsus bisferiens
(B) Watson's water hammer pulse
(C) Peaked T waves
(D) Equalization of central pressures
(E) Pulsus alternans

16. A 67-year-old male is diagnosed with a type B aortic dissection. At the time of initial presentation on the previous day, his blood pressure was $178 / 110 \mathrm{mmHg}$. He was treated with intravenous beta-blocker, and his blood pressure was reduced to $112 / 60 \mathrm{mmHg}$ and has remained in that range. However, one day later, he suddenly develops severe abdominal pain. His blood 
pressure is measured to be $110 / 56 \mathrm{mmHg}$. Which of the following is the most likely explanation?
(A) C. difficile infection
(B) Occlusion of the superior mesenteric artery
(C) Pancreatitis
(D) Aortoenteric fistula
(E) Diverticulitis

17. A 40-year-old male presents with acute chest pain and nausea. Serum troponin levels are elevated, and the ECG demonstrates ST segment elevation. Which of the following would be the strongest contraindication to intravenous thrombolytic therapy?
(A) Right knee arthroscopic surgery 1 month ago
(B) Recently completed antibiotic course for $H$. pylori infection
(C) Wide mediastinum on CXR
(D) History of alcohol abuse
(E) Endovascular aortic aneurysm repair 1 month ago 


\section{Answers}

\section{Answer $E$}

Proceeding with elective surgery 1 week after an acute $\mathrm{MI}$ is inappropriate (A-D). Patients with a recent $\mathrm{MI}$ are at significantly increased cardiac risk during noncardiac surgery, particularly within the first month after MI. Since the proposed operation is elective, options A-D would place the patient under unnecessary risk. Although performing the operation under local anesthesia with sedation (B) seems appealing, there is still considerable stress and cardiac risk with such an approach. The best recommendation for this patient is to postpone surgery for at least 4 weeks. At that point, consideration should still be given to cardiac stress testing prior to surgery or even further surgical delay, as the cardiac risk persists for at least 6 months after an MI.

\section{Answer A}

Major predictors of adverse postoperative cardiac events must be identified prior to elective noncardiac surgery. These include recent (within 1 month) MI, unstable or severe angina, decompensated CHF, and significant arrhythmias. Such cardiac conditions require postponing surgery and performing further cardiac workup. A systolic, crescendodecrescendo murmur at the sternal border of the right second intercostal space radiating into the neck is highly suggestive of aortic stenosis and would require an echocardiogram to rule out severe aortic stenosis. Aortic stenosis impairs coronary perfusion, which can become further exacerbated during induction of anesthesia. From all the choices listed, it portends the highest operative risk. Lee's revised cardiac risk index identifies intermediate risk factors; these include known coronary artery disease (B) history of CHF, history of stroke or TIA, insulin-dependent diabetes (C), creatinine $>2.0 \mathrm{mg} / \mathrm{dl}$ (possibly D), and high-risk surgery (i.e., aortic). Adding a point for each factor and a assigning a score (from 0 to 6 ) are highly effective in stratifying cardiac risk. Interestingly, smoking (E) has not been shown to be an independent risk factor for adverse perioperative cardiac events in most studies.

\section{Answer $\mathrm{E}$}

The patient has suffered a postoperative NSTEMI. Most NSTEMI (as opposed to a STEMI) in the postoperative setting are managed without percutaneous coronary intervention (PCI) with a combination of oxygen, morphine for pain relief, aspirin, and a beta-blocker. Optimally, an additional antiplatelet agent (such as clopidogrel) and intravenous heparin are also given, but this depends on how recent the operation was and the potential for postoperative bleeding. Consideration should be given to stress testing at 4-6 weeks after surgery, and depending on the results, PCI is then considered. Urgent PCI $(B, C)$ is indicated in the setting of a STEMI, and in certain high-risk NSTEMIs (continued rise in troponins, ongoing chest pain), but will require clopidogrel (again may not be desirable so soon after surgery) if a stent is placed. The patient described has a down trend of troponins and relief of symptoms, further supporting medical management. Emergent CABG (D) would be considered if PCI fails or is not technically feasible with severe threevessel disease. Emergent operations for acute MI continue to have a high mortality despite many technological advances in myocardial protection. Thrombolytic therapy (A) is an alternative when PCI is not available but would be contraindicated within 2-3 weeks of major surgery.

\section{Answer D}

The patient likely has hypertrophic obstructive cardiomyopathy, an asymmetric thickening of the ventricular septum that creates a narrowing of the left ventricular outflow tract. Vigorous exercise places him at increased risk of sudden cardiac death. T wave inversion (A) would be found in ischemic heart disease, very unlikely in an otherwise healthy 17-yearold. Laterally displaced PMI (B) would be found in patients with congestive heart failure, also very unlikely in this patient. Weak femoral pulses compared to brachial pulses (C) is a finding in coarctation of the aorta, and would not create the characteristic murmur. Murmurs due to aortic regurgitation, mitral regurgitation, and ventricular septal defect (VSD) increase in intensity with squatting (E).

\section{Answer D}

Acute pericarditis is inflammation in the pericardial sac accompanied by pericardial effusion. It can occur following post-MI (termed Dressler's syndrome), chest radiation, or recent heart surgery. Patients present with pleuritic chest pain that lessens when leaning forward, friction rub heard on auscultation, global ST elevation, and PR depression. Patients with myocarditis (A) usually present with signs and symptoms of acute decompensating heart failure (e.g., tachycardia, gallop, mitral regurgitation, and edema). Chest pain accompanied with MI (C) would not be expected to lessen with leaning forward. Furthermore, global ST elevation would not be expected. Cardiac tamponade (C) can occur once the effusion reaches a critical mass in which cardiac output is compromised. Pulmonary embolism (E) can present with pleuritic chest pain, but it will not be influenced by positioning and is more likely to have ECG findings suggestive of right heart failure.

\section{Answer C}

It is important to know the timing of causes of death after MI. In the first $48 \mathrm{~h}$ after MI, death is likely due to ventricular arrhythmia. If arrhythmia occurs after $48 \mathrm{~h}$, an implantable defibrillator should be placed. Ruptures of the myocardium, either as a ventricular septal rupture or free wall rupture (A), usually do not occur until 4-5 days after MI, 
at which point the dead myocardium has been weakened by the body's inflammatory response. Post-MI pericarditis, also known as Dressler's syndrome, (D) usually occurs weeks or months after MI or cardiac surgery. An embolic stroke (B) would present with sudden onset of numbness on one side of the body, cranial nerve deficits, and/or aphasia. It is unlikely to cause death so quickly. There is no reason to believe the patient has sustained an overwhelming infection $(\mathrm{E})$.

\section{Answer A}

The MUGA scan is the most accurate test in measuring ejection fraction. It is a noninvasive nuclear test that uses a radioactive isotope called technetium to evaluate the function of the ventricles. Though not as accurate, an echocardiogram (B) is used more commonly because it is cheaper and more readily available and can look for valve function as well as focal areas of wall motion abnormality. Electrocardiogram (C) and exercise stress test are unable to measure a patient's ejection fraction. Coronary angiography (D) is considered the gold standard in identifying coronary artery disease and can estimate ejection fraction, but is not as accurate.

\section{Answer E}

It is important to rapidly identify Stanford type A dissections, as they require urgent surgical intervention due to the fact that they can lead to cardiac tamponade, acute aortic valve insufficiency, acute MI, and stroke. A Stanford A dissection involves the ascending aorta and/or the aortic arch. Thus an aortic dissection involving the innominate artery is a Stanford type A. Stanford type B aortic dissection is more common. A Stanford type B dissection begins in the descending aorta, distal to the takeoff of the left subclavian artery (A-D). Stanford Type B dissections are much less likely to cause acute complications since the ascending aorta/aortic arch are not involved. A type B dissection may involve the mesenteric, renal, or iliac arteries, but not occlude them, as blood may continue to flow normally (either though the true or the false lumen). Most can be managed medically with blood pressure control (beta-blockers). Surgical intervention is needed if the involvement of these vessels leads to malperfusion (such as leg ischemia, bowel ischemia, or renal failure).

\section{Answer C}

Based on the description of the site of the dissection, this is a type B aortic dissection. These are usually managed medically (A) unless the patient has evidence of malperfusion. Since her peripheral pulses are all $2+$ and her abdomen is soft and non-tender, there is no evidence of malperfusion. The goal is to maintain a relatively low blood pressure in order to minimize stress on the aorta. Aggressive IV fluids (B) will not reduce blood pressure and may actually raise it.
Nicardipine (E) will lower blood pressure, but intravenous beta-blocker is the treatment of choice because it also reduces the rate of pressure increase with each beat of the heart, which lowers the stress on the aortic wall. Endovascular therapy (D) is not routinely needed for most type B dissections.

\section{Answer A}

Patients with a recent history of myocardial infarction are at risk of thrombus formation on the scarred endocardium, which can then embolize to the brain and cause a stroke. Patients with a recent history of MI and evidence of thrombus on echocardiography should be treated with warfarin to maintain an INR of 2-3 and followed up within 3 months. Thromboembolism from the left atrial appendage (D) is a concern in patients with atrial fibrillation. Paradoxical venous thromboembolism $(\mathrm{E})$ is a concern in patients with an atrial septal defect or patent foramen ovale, wherein a deep venous thrombus can travel through the defect into the left heart and ultimately to the brain. Septic embolism (B) is a concern in IV drug abusers and can lead to cerebral abscess. Type A dissection (C) would usually present with severe chest pain radiating to the back.

\section{Answer A}

This patient has a postoperative right-sided MI, resulting in compromised cardiac output secondary to decreased preload. One of the steps in management of right-sided MI is to administer fluids to help increase filling of the heart. Avoid nitrates $(\mathrm{B}, \mathrm{C})$ in these patients as it may further reduce preload. Acutely, patients with MI need oxygen, aspirin, analgesics, and beta-blockers. Dihydropyridine calcium channel blockers, such as nifedipine (D), are contraindicated in MI because of the associated peripheral vasodilation that may lead to reactive tachycardia and subsequently result in even more stress on the heart. ACE inhibitors (E) should be considered for long-term treatment after the acute episode has resolved.

\section{Answer D}

This patient's presentation is most concerning for acute mediastinitis. This is a life-threatening infection of the mediastinum with a very high mortality rate that is most commonly associated with cardiac surgery. The incidence rate is 1-2\% following $\mathrm{CABG}$. The source of infection may be a sternal wound infection, combined with instability of the sternum that permits bacteria to enter the mediastinum. Hamman's sign is a crunching sound heard with a stethoscope over the precordium during systole and is suggestive of acute mediastinitis. Patients will frequently present with chest pain, increased drainage from sternal wound, fevers, and leukocytosis. Chest radiograph findings include pneumomediastinum and/or air-fluid levels within the mediastinum. A CT scan can also support the diagnosis by demonstrating dehiscence of the 
sternum and stranding, fluid and air pockets within the anterior mediastinum. Management includes surgical debridement, drainage, antibiotics, and rewiring the sternum. Acute pericarditis (A) will present with pleuritic chest pain that lessens when leaning forward, friction rub heard on auscultation, and characteristic ECG findings (global ST elevation). Pneumonia (E) would present with shortness of breath, productive cough, and abnormal lung sounds. Postoperative MI (B) would not be expected to present with evidence of systemic inflammation. Empyema (C) is defined as pus in the pleural space, and would not explain the physical exam findings of sternal instability and Hamman's sign. CT scan would demonstrate a loculated fluid collection within the right or left pleural cavity.

\section{Answer B}

The classic signs of severe aortic stenosis are angina (A), syncope (C), and congestive heart failure (which may manifest as swollen legs). Of the three, congestive heart failure portends the worst prognosis, with median survival as low as 2 years. A loud mid-systolic murmur (D) indicates hemodynamically significant obstruction but is a better prognostic sign than an absent murmur, which indicates low blood flow across the valve. Small head nodding movements with each heartbeat (E) are known as de Musset's sign and is found in aortic regurgitation.

\section{Answer D}

All of the above are risk factors for aortic dissection (A-C, E). However, the most significant risk factor for aortic dissection is systemic hypertension.

\section{Answer D}

In cardiac tamponade, fluid (blood or effusion) in the pericardial space externally compresses the heart, which limits diastolic filling and reduces stroke volume. Since pericardial fluid is free flowing, the pressure is distributed equally along the pericardium. As this continues the rising pressure in the pericardium is transmitted to all four cardiac chambers resulting in equalization of central pressures. Pulsus bisferiens (A), also known as a biphasic pulse, refers to two strong systolic pulses with a mid-systolic dip, in other words, two pulses during systole. It can be seen in aortic regurgitation with or without aortic stenosis and hypertrophic cardiomyopathy. Watson's water hammer pulse (B) is a pulse with a rapid upstroke and descent seen in patients with aortic regurgitation. Peaked T waves (C) is most often associated with hyperkalemia. It is unlikely to be seen in patients with cardiac tamponade since their ECG findings are characteristically low voltage. Pulsus alternans (E) is a physical exam finding wherein the amplitude of a peripheral pulse changes from beat to beat associated with changing systolic blood pressure. It is most commonly caused by left ventricular failure.

\section{Answer B}

Sudden onset of severe abdominal pain in association with an aortic dissection should always raise suspicion for malperfusion of the bowel which can lead to bowel gangrene and death. This most likely would occur if the dissection extends into, and suddenly occludes, the superior mesenteric artery, which supplies blood to the bowel from the ligament of Treitz to the mid-transverse colon. It is also important to recognize that bowel ischemia early on causes excruciating pain in the absence of peritonitis ("pain out of proportion to physical exam"). He has not been on broad-spectrum antibiotics, and has no reason to have $C$. difficile infection (A), which most often presents with vague abdominal pain and diarrhea. Pancreatitis (C) presents with epigastric pain radiating to the back, nausea, vomiting, anorexia, fever, and tachycardia and is most commonly associated with cholelithiasis and alcohol abuse. Aortoenteric fistula (D) is a possible long-term sequela in patients who have had an intra-aortic synthetic graft placed. Diverticulitis (E) is a common cause of left lower quadrant abdominal pain in elderly patients, and does not typically cause such sudden severe pain.

\section{Answer C}

Wide mediastinum on chest X-ray is concerning for aortic dissection. Patients with type A aortic dissection can present with coronary artery malperfusion and thus have a similar presentation as an acute MI. Suspected aortic dissection is considered an absolute contraindication to thrombolysis in patients with myocardial infarction. The remaining choices (A-B, D-E) are all relative contraindications for intravenous thrombolytics. 


\section{Endocrine}

Areg Grigorian, Masha J. Livhitz, Christopher M. Reid, Michael W. Yeh, and Christian de Virgilio

\section{Questions}

1. A 27-year-old woman has 3 months of intermittent spells of severe headache, heart palpitations, and sweating. A pregnancy test at her primary care doctor's office is positive. Further workup reveals that her plasma metanephrine level is $220 \mathrm{pg} / \mathrm{ml}$ (normal $12-60 \mathrm{pg} / \mathrm{ml}$ ). What is the next step in establishing the diagnosis?
(A) $\mathrm{CT}$ abdomen
(B) Repeat plasma metanephrine level after the patient has delivered
(C) MRI abdomen
(D) I $^{131}$-MIBG scan
(E) Reassure patient that symptoms are related to pregnancy

2. Preoperative medical optimization for a patient with a pheochromocytoma routinely includes:
(A) Octreotide drip for $24 \mathrm{~h}$ before surgery
(B) Control of hypertension with beta-blockade as first- line agent
(C) Control of hypertension with alpha-blockade as first-line agent
(D) Metyrosine
(E) Diuretics for blood pressure management

3. A 55-year-old otherwise healthy patient undergoes a non-contrast CT abdomen to evaluate for possible kidney stones and is incidentally noted to have a $8 \mathrm{~cm}$ mass in the left adrenal gland. The mass has irregular borders and high attenuation, suggesting a lipid-poor lesion, and appears to be adherent to the kidney. How should this patient be managed?
(A) Observation with repeat CT scan in 3 months
(B) Open adrenalectomy
(C) Laparoscopic adrenalectomy
(D) Radiation therapy
(E) Percutaneous biopsy

4. A 50-year-old female has been recently diagnosed with primary hyperparathyroidism. She comes in to her doctor complaining of increased bone pain in her legs. She is found to have elevated serum calcium, alkaline phosphate, and PTH. Her doctor decides to order plain films of her lower extremities. The radiographs show very thin bones with a stress fracture and bowing of both femur bones. She also has characteristic cysts with a motheaten appearance. What is the most likely diagnosis?
(A) Osteoporosis
(B) Osteopetrosis
(C) Osteomalacia
(D) Osteitis fibrosa cystica
(E) Paget's disease of the bone

5. A 60-year-old man is found to have a $3 \mathrm{~cm}$ right adrenal mass on CT scan which was obtained a month earlier following a MVC. He is asymptomatic, and does not report a history of hypertension or diabetes. What is the most appropriate next step in management?
(A) Repeat CT scan in 6 months
(B) Percutaneous needle biopsy
(C) Biochemical workup for hormone excess
(D) Laparoscopic adrenalectomy
(E) No further follow-up is necessary

6. An elderly nursing home patient has been bedridden for several months due to a series of debilitating strokes. Past medical history is significant for hypertension, controlled with a diuretic, and Paget's disease. Recently, the patient has been complaining of vague abdominal pain, constipation, and depressed mood. On physical examination, the patient is alert and oriented. Abdominal examination is unremarkable. Which of the following electrolyte abnormalities would most likely explanation her symptoms?
(A) Hyponatremia
(B) Hypernatremia
(C) Hyperphosphatemia
(D) Hypocalcemia
(E) Hypercalcemia

7. Which of the following is most consistent with an aldosterone-secreting adrenal adenoma?

(A) Hyperglycemia, hirsutism, and abdominal striae

(B) Hypertension and hyperkalemia

(C) Hypertension and hypokalemia

(D) Elevated plasma metanephrine and hypertension

(E) Increased vanillylmandelic acid excretion and hypertension

8. A 35-year-old patient presents for a follow-up visit for an elevated serum calcium level of $12.8 \mathrm{mg} / \mathrm{dL}$ and an elevated PTH. He is a thin man without a significant past medical history. He reports that for the past 2 weeks he has been experiencing loose stools, polydipsia, and polyuria. On physical exam he was found to have large erythematous erosions with blisters over the lower abdomen. Which tumor would best explain the patient's symptoms and rash?
(A) Insulinoma
(B) Prolactinoma
(C) VIPoma
(D) Glucagonoma
(E) Adrenal adenoma 
9. A 32-year-old female patient arrives for follow-up for new-onset hypertension. She was started on hydrochlorothiazide 6 months ago. During her visit, she was found to have a blood pressure of $152 / 98 \mathrm{mmHg}$. She also complains of recent episodes where she experiences sudden palpitations, chest pain, diaphoresis, headache, and anxiety. Her laboratory exam demonstrates a calcium of $13.2 \mathrm{mg} / \mathrm{dl}$ (normal $8.5-10.2 \mathrm{mg} / \mathrm{dl}$ ), PTH of $102 \mathrm{pg} / \mathrm{ml}(10-55 \mathrm{pg} / \mathrm{ml})$, and an elevated plasma metanephrine. Which of the following would be an important additional component in the workup?
(A) Fasting blood glucose
(B) Prolactin level
(C) MRI of the sella turcica
(D) Serum calcitonin
(E) Serum gastrin level

10. A 45-year-old man has had hazy vision for the past month, particularly when he is driving at night. He also endorses small rubberlike nodules on the skin of his trunk, back, arms, and legs that are not painful and do not itch. After seeing his ophthalmologist, he is diagnosed with bilateral cataracts and is scheduled to receive elective cataract surgery. During induction of anesthesia, following intubation, the patient's pressure increases from $110 / 70$ to $200 / 90 \mathrm{mmHg}$. $\mathrm{PaCO}_{2}$ is nor$\mathrm{mal}$ as is his $\mathrm{pH}$. His temperature is $101.5^{\circ} \mathrm{F}$. An esmolol drip is immediately instituted, after which BP increases to 220/90 $\mathrm{mmHg}$ and an ECG shows T wave inversion. What is the most likely underlying etiology?
(A) Intra-abdominal tumor
(B) Malignant hyperthermia
(C) Thyrotoxicosis
(D) Inadequate anesthetic agent
(E) Undiagnosed pituitary tumor

11. A 12-year-old boy presents to the doctor for a lump in his neck. He is healthy with no previous medical problems. On physical examination, he has a well-defined anterior neck mass, located in the midline and above the cricoid cartilage. The mother states that she has noted the lesion since he was about 2 years old. It does not bother him. On physical examination, the mass elevates with swallowing and is non-tender. He has no cervical adenopathy and no other complaints. The neck mass is described as a hypoechoic mass on ultrasonography. A subsequent thyroid scintogram is performed and confirms the thyroid gland is in its correct anatomic position. Which of the following would be recommended next for this mass?
(A) FNA biopsy
(B) Proceed to surgical excision
(C) Reassurance and observation
(D) $\mathrm{TSH}$ and free $\mathrm{T} 4$
(E) CT scan

12. In addition to elevated plasma free metanephrine, a change in what other serum marker can help support the diagnosis of pheochromocytoma?
(A) Plasma chromogranin A
(B) Plasma superoxide dismutase
(C) Malondialdehyde
(D) CA 19-9
(E) 5-Hydroxyindoleacetic acid (HIAA)

13. A 42-year-old man with a family history of endocrine tumors is diagnosed with MEN-2A after presenting with uncontrolled hypertension and subsequent genetic workup. He was found to have a right adrenal pheochromocytoma and asymptomatic hyperparathyroidism. What is the recommended surgical management for this patient?

(A) Parathyroid surgery first, followed by adrenalectomy

(B) Adrenalectomy first, followed by parathyroid surgery

(C) Medical conditioning for 2 weeks prior to adrenalectomy, followed by parathyroid surgery

(D) Medical conditioning for 2 weeks prior to simultaneous parathyroid surgery and adrenalectomy

(E) Medical conditioning for 2 weeks followed by adrenalectomy only

14. A 39-year-old man is recovering from bilateral adrenalectomy for a pheochromocytoma. On his second postoperative day, he begins to complain of nausea, vomiting, weakness, blurry vision, and mild abdominal pain. His temperature is $102.9{ }^{\circ} \mathrm{F}$, and blood pressure is 90/68 $\mathrm{mmHg}$. His ECG shows sinus tachycardia. His laboratory examination from that morning showed:

Sodium: $134 \mathrm{mEq} / \mathrm{L}(137-145 \mathrm{mEq} / \mathrm{L})$

Potassium: $5.8 \mathrm{mEq} / \mathrm{L}(3.6-5.0 \mathrm{mEq} / \mathrm{L})$

Calcium: $7.4 \mathrm{mg} / \mathrm{dL}(8.9-10.4 \mathrm{mg} / \mathrm{dL})$

BUN: $12 \mathrm{mg} / \mathrm{dL}(7-21 \mathrm{mg} / \mathrm{dL})$

Creatinine: $1.2 \mathrm{mg} / \mathrm{dL}(0.5-1.4 \mathrm{mg} / \mathrm{dL})$

Glucose: $70 \mathrm{mg} / \mathrm{dL}(65-110 \mathrm{mg} / \mathrm{dL})$

Albumin: $2.4 \mathrm{~g} / \mathrm{dL}(3.5-4.8 \mathrm{~g} / \mathrm{dL})$

WBC $10.5 \times 103 / \mu \mathrm{L}(4.1-10.9 \times 103 / \mu \mathrm{L})$

Which of the following can best explain this patient's current presentation? 

(A) Volume depletion
(B) Sepsis
(C) Hypocalcemia
(D) Low cortisol
(E) Loss of catecholamine production

15. A 56-year-old woman is recovering after undergoing total thyroidectomy for papillary carcinoma. Her temperature is $99.8^{\circ} \mathrm{F}$, blood pressure is $120 / 80 \mathrm{mmHg}$, and pulse is $90 / \mathrm{min}$. During her postoperative examination by the intern, the patient complains of numbness and tingling around her mouth and in her hands and feet. What could have been done postoperatively to anticipate and potentially remedy these symptoms?
(A) Check magnesium
(B) Check parathyroid hormone
(C) Check potassium
(D) Check TSH and free T4
(E) Carotid ultrasound

16. A 42-year-old man presents with new-onset hypertension and elevated hemoglobin $(19 \mathrm{mg} / \mathrm{dL})$ and hematocrit $(58 \%)$ levels on subsequent laboratory examination. A CT scan demonstrates bilateral adrenal masses suspicious for pheochromocytoma. His elevated hemoglobin and hematocrit are believed to be secondary to a paraneoplastic syndrome. What other tumor is classically associated with this same paraneoplastic syndrome?
(A) Glioblastoma multiforme
(B) Hemangioblastoma
(C) Colorectal cancer
(D) Wilms' tumor
(E) Osteosarcoma

17. Which of the following is true regarding paragangliomas (extra-adrenal pheochromocytomas)?

(A) The most common location is within the kidney

(B) There is a decreased association with familial syndromes (e.g., MEN-2, Von Hippel-Lindau) compared to pheochromocytomas

(C) They are less likely to be malignant compared to pheochromocytomas

(D) Functional imaging (MIBG) is particularly useful to diagnose metastatic disease, particularly when $\mathrm{CT} / \mathrm{MRI}$ are negative

(E) They are different on a cellular level from intraadrenal pheochromocytomas

18. A malignant pheochromocytoma is diagnosed by:

(A) Pathologic identification of high mitotic rate, cellular atypia, and capsular invasion

(B) Positive MIBG scan
(C) Presence of metastasis at sites normally devoid of chromaffin tissue

(D) Biomolecular markers

(E) The presence of intractable hypertension

19. A 45-year-old female presents with a $2 \mathrm{~cm}$ painless mass in her right anterior neck that has been present for 3 months and slowly enlarging. On physical exam, the mass feels firm and moves up and down with swallowing. She denies weight loss, weight gain, heat intolerance, or anxiety. A serum TSH level is normal. The most important step in the workup is:
(A) CT scan of the neck
(B) MRI of the neck
(C) Fine-needle aspiration (FNA)
(D) Open biopsy
(E) Nuclear scan

20. Three hours after total thyroidectomy for thyroid cancer, the patient complains of difficulty breathing. On physical examination, the patient has stridor and appears to be in moderate respiratory distress. Examination of the wound demonstrates tense swelling. The next step in the management is:
(A) Immediately reopen wound at the bedside
(B) Intubation
(C) Emergent return to the operating room for wound exploration
(D) Check oxygen saturation
(E) Send arterial blood gas

21. During the course of a total thyroidectomy in a 40-yearold female, the surgeon divides the superior thyroid artery and vein in one large ligature. After dividing the vascular pedicle, the surgeon notices that it appears that a nerve was transected. The surgeon postoperatively should warn the patient that she will most likely have:
(A) Permanent hoarseness
(B) A droop in the corner of her mouth
(C) Difficulty swallowing
(D) Trouble hitting high notes when singing
(E) A need for a permanent tracheostomy

22. A 45-year-old female presents to her physician complaining of abdominal pain. She has a history of recurrent kidney stones and was recently discharged from the hospital after undergoing ureteroscopic laser lithotripsy. Her laboratory examination is significant for calcium of $13.6 \mathrm{mg} / \mathrm{dL}$ (normal $8.5-10.2 \mathrm{mg} / \mathrm{dL}$ ) and PTH of $112 \mathrm{pg} / \mathrm{mL}(10-55 \mathrm{pg} / \mathrm{mL})$. She is scheduled for operative management of her underlying condition. At surgery, all four parathyroid glands are identified. Only one appears to be abnormally enlarged and is 
removed. Confirmation of curative resection is best achieved via:
(A) Intraoperative ultrasound
(B) Intraoperative frozen section
(C) Intraoperative PTH levels
(D) Immediate postoperative serum calcium level
(E) Postoperative sestamibi scan

23. A 35-year-old female presents with bone pain, abdominal pain, and depressed mood. Her laboratory examination is significant for calcium of $11.3 \mathrm{mg} / \mathrm{dL}$ (normal $8.5-10.2 \mathrm{mg}$ / $\mathrm{dL})$ and PTH of $109 \mathrm{pg} / \mathrm{ml}(10-55 \mathrm{pg} / \mathrm{mL})$. Localization of the enlarged gland or glands is best achieved by:
(A) Preoperative MRI
(B) Preoperative ultrasound
(C) Preoperative sestamibi scan

(D) Preoperative FNA

(E) Intraoperative exploration of all four glands

24. A 38-year-old female arrives for her yearly physical. She has no complaints but was incidentally found to have laboratory markers suggestive of primary hyperparathyroidism. Subsequent workup reveals involvement of all four parathyroid glands. She remains asymptomatic. What is the best recommendation for management of this patient?
(A) Observation
(B) Surgical removal of all four glands
(C) Surgical removal of 3.5 glands
(D) Biochemical monitoring of serum calcium and serum creatinine annually
(E) Cinacalcet 


\section{Answers}

\section{Answer $C$}

This patient presents with the rare but classic presentation of pheochromocytoma during pregnancy. The preferred imaging modality in pregnancy is an MRI, due to the risks of exposing the fetus to radiation with other types of imaging (A, D). In men and non-pregnant women, CT with contrast can also be considered a first line imaging study. Pheochromocytoma is usually hyperintense on T2-weighted images due to its high water content. Failing to work up and treat a potential pheochromocytoma in pregnancy exposes the fetus and mother to a very high risk of morality during the pregnancy and delivery $(\mathrm{B}, \mathrm{E})$.

\section{Answer C}

Patients with pheochromocytoma are volume depleted due to intense alpha-mediated vasoconstriction. Hypertension is controlled with alpha-blockade (e.g., phenoxybenzamine) for 10-14 days before surgery. This allows for volume expansion, and the patient is encouraged to liberally intake salt and fluids. The dose is titrated until hypertensive episodes are controlled, often resulting in mild orthostatic hypotension. Beta-blockers (B) can be used to decrease reflex tachycardia once appropriate alpha-blockade has been established. Initiating beta-blocker therapy prematurely can precipitate a hypertensive crisis due to unopposed alpha-adrenergic vasoconstriction. Octreotide (A) is a somatostatin analogue that may have minimal efficacy in the palliation of symptoms from malignant pheochromocytoma, but it has no role in preparing a patient for surgery. Metyrosine (D) inhibits catecholamine production and is a secondary agent for pheochromocytoma, though now rarely used. Diuresis (E) would be contraindicated as these patients are volume depleted.

\section{Answer B}

This patient was incidentally found to have an adrenal mass. Guidelines for surgical resection include tumors $>6 \mathrm{~cm}$, features on CT suspicious for malignancy (high attenuation, irregular borders, inhomogeneous), and those that are hormonally active. Most adrenal carcinomas are hormonally active. Thus the patient described has several indications for adrenalectomy. Open adrenalectomy is preferred when malignancy is suspected, as this allows for a wider resection with en bloc resection if adjacent structures are involved and eliminates the possibility of seeding the port sites that may occur with laparoscopic adrenalectomy (C). Laparoscopic adrenalectomy is preferred for benign lesions. Radiation therapy (D) is not the mainstay of treatment for adrenal cortical carcinoma. Percutaneous biopsy (E) is not recommended as there are no histologic features that diagnose adrenal cortical carcinoma and a biopsy may risk seeding the biopsy tract.

\section{Answer D}

Osteitis fibrosa cystica is a skeletal disorder that results from a surplus of parathyroid hormone. Patients experience increased bone pain, bone fractures, and skeletal deformities with bowing of the bones. Radiographs show thin bones, fractures, and cysts with a moth-eaten appearance. Osteoporosis (A) usually occurs in elderly patients and is characterized by decreased bone density with normal mineralization. It does not have any associated cyst-like features. Similarly, osteopetrosis (B) would not have any cysts seen on plain films. Paget's disease (E) results from overactive osteoclasts and osteoblasts leading to excessive bone turnover and is characterized by tibial bowing, kyphosis, increased cranial diameter, and deafness. Patients with Paget's disease and osteoporosis have normal serum calcium, while patients with osteomalacia (C) would be expected to have decreased serum calcium.

\section{Answer C}

The first step in the evaluation of an incidentally discovered adrenal mass is to perform a biochemical workup to determine if the tumor is functional or nonfunctional $(\mathrm{E})$. In practice, it is common to order a single battery of tests: serum aldosterone, plasma renin activity, and a 24-h urine collection to simultaneously measure catecholamines, metanephrines, and cortisol. Given that this patient is normotensive, the suspicion for pheochromocytoma and hyperaldosteronism is low. In addition, adrenal masses $<6 \mathrm{~cm}$ are unlikely to be malignant. If the mass is found to be a hormonally active adrenal adenoma, then laparoscopic adrenalectomy (D) would be recommended. If biochemical testing reveals a nonfunctioning mass, this small lesion may be observed with interval CT scanning (A). Percutaneous needle biopsy (B) cannot readily distinguish between benign and malignant primary adrenal tumors.

\section{Answer E}

Hypercalcemia can cause abdominal pain, constipation, mental status changes, and depressed mood (stones, bones, moans and groans). Prolonged immobilization is a known cause of hypercalcemia and is seen in adolescents and in other patients with increased bone turnover such as Paget's disease. Certain diuretics (thiazide) also cause hypercalcemia by increasing renal calcium resorption.

\section{Answer $\mathrm{C}$}

Patients with hyperaldosteronism have hypertension and hypokalemia - not hyperkalemia (B). Aldosterone acts on the kidney to increase sodium reabsorption, and potassium is excreted to balance the positively charged sodium ions. Hyperglycemia, hirsutism, and abdominal striae (A) are more consistent with Cushing's syndrome. Elevated plasma metanephrine, hypertension, and increased vanillylmandelic 
acid excretion (D, E) are all consistent with pheochromocytoma.

\section{Answer D}

Elevated serum calcium combined with elevated PTH is consistent with primary hyperparathyroidism. Rarely, it can be associated with MEN-1 which includes parathyroid, pituitary, and pancreatic pathology (3Ps). Pancreatic tumors include gastrinoma, insulinoma, VIPoma, and glucagonoma. Glucagonoma should be suspected in a patient with newonset diabetes mellitus (even if thin), diarrhea, and the classic rash: annular, erythematous erosions with blisters over the lower abdomen (necrolytic migratory erythema). The patient's symptoms of polyuria and polydipsia are highly suggestive of diabetes mellitus. Insulinoma (A) is characterized by hypoglycemia, headache, visual changes, confusion, weakness, and diaphoresis. Prolactinomas (B) are excess prolactin-producing anterior pituitary tumors that may result in amenorrhea, galactorrhea, decreased libido, and gynecomastia. A VIPoma (C) (also called WDHA syndrome: watery diarrhea hypokalemia achlorhydria) presents with profuse diarrhea, but will not have any skin manifestations of the disease. An adrenal adenoma (E) is oftentimes benign, nonfunctional, and incidentally found on imaging (incidentalomas).

\section{Answer D}

Severe hypertension in a young patient should raise suspicion for surgically correctable causes such as aldosteronoma, Cushing's disease, coarctation of the aorta, fibromuscular dysplasia of the renal arteries, and pheochromocytoma. Her symptoms, combined with an elevated plasma metanephrine level, make pheochromocytoma the most likely cause. The addition of labs consistent with primary hyperparathyroidism (elevated calcium and PTH) suggests she has MEN-2A which is characterized by primary hyperparathyroidism, pheochromocytoma, and medullary thyroid cancer. Calcitonin is a reliable tumor marker for medullary thyroid cancer and should always be ordered to rule out this very aggressive cancer in this patient population. Fasting blood glucose (A) (insulinoma), prolactin levels (prolactinoma) (B), MRI of the sella turcica (C) (pituitary adenoma), and serum gastrin level (E) (gastrinoma) are all associated with MEN-1.

\section{Answer A}

A sudden rise in blood pressure after anesthetic induction raises concern for an undiagnosed pheochromocytoma, malignant hyperthermia, and thyrotoxicosis (thyroid storm). For each of these situations, cessation of anesthesia is recommended. There are several clues that point to pheochromocytoma as the cause. The administration of beta-blockers without alpha-blockade first leads to worsening hypertension due to unopposed alpha-mediated vasoconstriction as in the case above. Pheochromocytoma is associated with neurofibromatosis-1 which may present with skin neurofibromas (rubberlike discolored skin lesions) and cataracts. Malignant hyperthermia (B) presents with muscle rigidity (most often the masseter), a rapid increase in core body temperature, a rise in end tidal $\mathrm{CO}_{2}$, arrhythmia, and a mixed metabolic and respiratory acidosis at anesthetic induction. Treatment is immediate cessation of surgery and dantrolene. Thyrotoxicosis (C) presents in a similar fashion to malignant hyperthermia (fever, hypertension, tachycardia); however, it is not associated with muscle rigidity or rising end tidal $\mathrm{CO}_{2}$. The associated hypertension and tachycardia respond to the administration of beta-blockade. It is due to a hypermetabolic state caused by excess thyroid hormone. Inadequate anesthetic agents (D) may lead to hypertension and tachycardia, but would not lead to high fevers. An undiagnosed pituitary tumor resulting in excess ACTH production can cause hypertension, but this will be accompanied with symptoms consistent with Cushing's disease (e.g., truncal obesity, abdominal striae, muscle wasting, hirsutism).

\section{Answer B}

This patient has a thyroglossal duct cyst, which is the most common midline congenital malformation of the neck. Though present at birth, these do not often appear until age 2 as baby fat recedes. During embryological development, the thyroid originates at the base of the tongue and travels down the thyroglossal duct to the anterior neck, where it normally involutes. However, if a persistent duct remains, it may undergo cystic dilation later in life and present as a welldefined anterior neck mass, located midline and above the cricoid cartilage. Unlike a brachial cleft cyst, this elevates with tongue protrusion or swallowing. Ectopic thyroid gland may be associated with thyroglossal duct cysts so it's necessary to confirm the thyroid gland is in its correct anatomic location prior to surgical intervention. The definitive management involves thyroglossal duct cyst excision or the Sistrunk procedure. Reassurance and observation (C) are inappropriate as thyroglossal duct cysts have a high rate of recurrent infections and a small risk of progressing to malignancy. FNA biopsy (A) is appropriate for a thyroid nodule, but not for suspected thyroglossal duct cyst. He does not have symptoms suggestive of hyper- or hypothyroidism so a thyroid panel would not be indicated (D). CT scan (E) is unnecessary for the diagnosis, and additionally should not be performed in such a young patient secondary to significant radiation exposure.

\section{Answer $A$}

Plasma free metanephrine is highly sensitive for pheochromocytoma but is more prone to false-positive results. Plasma chromogranin A is released from neuroendocrine cells and is elevated in the majority of patients with pheochromocytoma. It is nonspecific (i.e., it is elevated in other neuroendocrine 
tumors) but can help confirm the diagnosis. Superoxide dismutase and malondialdehyde $(\mathrm{B}, \mathrm{C})$ are both markers for oxidative stress, and neither has been shown to be associated with pheochromocytoma. CA 19-9 (D) may be elevated in some patients with pancreatic cancer. Increased level of 5-hydroxyindoleacetic acid (HIAA) (E) would be expected in a patient with carcinoid syndrome.

\section{Answer $C$}

Patients with MEN-2A can develop pheochromocytoma, hyperparathyroidism, and medullary thyroid cancer. The definitive management for pheochromocytoma consists of medical conditioning with alpha-blockade and sometimes beta-blockade for at least 2 weeks, followed by an adrenalectomy (B). This should be performed first (A, D-E) because a pheochromocytoma can increase the risk of complications during the surgical management of other endocrine tumors. Although he is asymptomatic with respect to his hyperparathyroidism, parathyroid surgery is generally recommended for most patients with inherited forms, as it tends to be more aggressive and presents at a much younger age. Age less than 50 is an indication for parathyroid surgery for sporadic forms as well, as the patient is more likely to suffer one of the sequelae of hyperparathyroidism.

\section{Answer D}

If a patient that has undergone bilateral adrenalectomy presents postoperatively with severe hypotension and hypoglycemia, suspect Addisonian crisis (acute adrenal insufficiency) and check a cortisol level. This is considered to be a lifethreatening condition caused by insufficient levels of cortisol, which is responsible for maintaining blood pressure and glucose homeostasis. Patients will present with nausea, vomiting, weakness, blurry vision, and mild abdominal pain. Laboratory exam would be expected to show hypoglycemia, hyperkalemia, and mild hyponatremia. Plasma ACTH levels will be low, and a Cortrosyn (synthetic ACTH) stimulation test will demonstrate a low cortisol response. This patient should receive immediate fluid resuscitation (normal saline) and intravenous corticosteroids. Acute adrenal insufficiency does not respond to vasopressors. Additionally, it can mimic sepsis. However, he does not meet SIRS criteria. Similarly, sepsis (B) is unlikely to present with this patient's lab abnormalities. Patients that have had major surgery should always be monitored for signs of internal hemorrhaging. Although his serum calcium is shown to be low (C), this should be corrected for hypoalbuminemia. His corrected serum calcium is $8.7 \mathrm{mg} / \mathrm{dL}$, is within the normal range, and would not explain the hypotension (B). Although he may be volume depleted (A), this would not cause hypoglycemia or hyperkalemia. Loss of catecholamine production (E) may accompany Addisonian crisis and is also seen after removing a pheochromocytoma. It is associated with hypotension and hypoglycemia; however, it will not cause hyperkalemia and hyponatremia.

\section{Answer B}

The patient most likely has hypocalcemia. Temporary hypoparathyroidism occurs in up to $30 \%$ of patients after total thyroidectomy and generally lasts a few weeks. It is thought to be related to temporary ischemia to the adjacent parathyroid glands. Patients will complain of numbness and tingling in their hands and feet, as well as around the mouth. These patients should be managed with prompt oral calcium supplementation. Oral calcitriol may be added to increase calcium absorption from the gut. Some centers routinely check the postoperative PTH level for the purposes of anticipating hypocalcemia. Left untreated, hypocalcemic symptoms may progress to muscle twitching (including Chvostek's sign) and ultimately tetany, which is an emergency. IV calcium (gluconate or chloride) may be given in these circumstances, but its use can generally be avoided when patients are carefully monitored postoperatively. Symptoms of hypomagnesemia (A) are indistinguishable from hypocalcemia; however, low magnesium levels are not associated with thyroidectomy. Disturbances in potassium (C) and thyroid hormone (D) would not cause the symptoms described. Carotid ultrasound (E) would be indicated if the patient developed symptoms of a stroke or transient ischemic attack (one-sided arm and leg weakness/numbness).

\section{Answer B}

This patient most likely has an ectopic production of erythropoietin leading to high levels of hemoglobin and hematocrit. This paraneoplastic syndrome, termed polycythemia vera, is classically associated with pheochromocytoma, renal cell carcinoma, hepatocellular carcinoma, and hemangioblastoma (A, D-E).

\section{Answer D}

Paragangliomas arise from extra-adrenal chromaffin tissue, with the most common location being in the abdomen (organ of Zuckerkandl). They are essentially identical on a cellular level to intra-adrenal pheochromocytomas. However, they are more likely to have a hereditary basis (30-50\% of cases) and to be malignant $(15-35 \%)$. The diagnosis is made by biochemical analysis followed by imaging localization. It is particularly important to consider a whole body functional scan due to the higher propensity for multifocal and metastatic disease.

\section{Answer C}

There is currently no way to establish the diagnosis of malignancy in pheochromocytoma based on histopathologic evaluation (A). However, there are tumor characteristics that are associated with higher risk (e.g., larger size, extra-adrenal 
location, certain genetic mutations, and a high tumor proliferative index). Malignancy is determined by the development of metastatic disease, defined by a recurrence in an area that normally does not have any chromaffin tissue (lymph nodes or a distant site such as the liver or lungs). MIBG scanning (B) can be useful to identify metastatic disease, but positivity of the primary tumor on MIBG does not determine whether it is malignant. Biomolecular markers (D) can differentiate a functional tumor from nonfunctional, but is unable to rule out malignancy. Similarly, intractable hypertension $(\mathrm{E})$ is not a characteristic of malignancy.

\section{Answer C}

The most important step in the diagnostic workup of a thyroid nodule is to obtain a tissue sample. This is best obtained via fine-needle aspiration and is best done under ultrasound guidance. Thyroid nodules greater than $1 \mathrm{~cm}$ in size, nodules with ultrasound characteristics suggestive of malignancy (internal microcalcifications, e.g.), or those with a history of growth should undergo ultrasound guided FNA. CT (A) or MRI (B) would be appropriate for patients found to have clinical or sonographic evidence of locally advanced thyroid cancer that may extend into the aerodigestive tract or substernal region. Open biopsy (D), done by removing an entire thyroid lobe, should be done next if FNA results are suspicious for a follicular neoplasm. Nuclear scanning $(E)$ has a very limited role in the preoperative setting. It is more beneficial in the postoperative setting to look for recurrent or metastatic malignancy.

\section{Answer A}

Don't forget the ABCs. This patient has a compromised airway and is in moderate respiratory distress. Normally, the first step to ensure an airway is via endotracheal intubation (B). However, a neck hematoma is in a closed space that leads to compression of the airway that may render safe intubation difficult or impossible. As such, the first step is to immediately open the neck wound at the bedside to decompress the hematoma. This will typically relieve the airway obstruction. The patient can then be transported emergently to the operating room for intubation, wound exploration, adequate hemostasis, and subsequent wound closure (C). Although thyroidectomy is considered a safe procedure, one well-known complication is airway obstruction following bleeding and hematoma formation which occurs within the first $24 \mathrm{~h}$ after thyroidectomy. Checking oxygen saturation (D) or waiting for labs (E) is never appropriate for a patient with a compromised airway.

\section{Answer D}

The superior laryngeal nerve lies adjacent to the superior thyroid artery and is thus at high risk of being injured during mobilization of the thyroid, particularly the superior pole.
The external branch of the superior laryngeal nerve permits singing in a high pitch. This nerve may be injured in up to $25 \%$ of cases but is usually asymptomatic unless the patient is a singer or voice professional. Damage to the recurrent laryngeal nerve on one side results in a paralyzed vocal cord in a median or paramedian position. This manifests as hoarseness (A) and sometimes aspiration. The rate of permanent unilateral recurrent laryngeal nerve injury during thyroidectomy should be less than $2 \%$ in expert hands. If both recurrent laryngeal nerves were injured during a total thyroidectomy, then both vocal cords could be paralyzed, and this may lead to a compromised airway which may necessitate a permanent tracheostomy $(\mathrm{E})$. A droop in the corner of the mouth results from injury to the marginal mandibular branch of the facial nerve. Swallowing is controlled by multiple nerves (C) including the glossopharyngeal, vagus, and/ or hypoglossal nerves.

\section{Answer C}

The surgical treatment of hyperparathyroidism depends on whether the pathology is a single adenoma (most common, remove single gland), more than one adenoma (remove abnormal ones), or four gland hyperplasia (remove 3.5 glands). Distinguishing these entities is not always obvious. Because of the short half-life of PTH (about $4 \mathrm{~min}$ ), intraoperative rapid PTH testing aids in determining the completeness of parathyroid resection. The most commonly used protocol involves drawing PTH levels at the time of gland excision and again 10 min post-excision. A fall of $>50 \%$ in the PTH level is associated with a $98 \%$ long-term cure rate. Given the small size of the parathyroid glands, it is generally not recommended to biopsy all of them for frozen section (B), as such a biopsy may render all the glands ischemic. Transient hypocalcemia is expected following parathyroidectomy so postoperative serum calcium level (D) is not indicative of cure. Oral calcium supplementation can help alleviate minor symptoms. Intraoperative ultrasound (A) is sometimes used when the abnormally enlarged gland cannot be found. Sestamibi (E) may be used if recurrent or persistent hyperparathyroidism develops, but is not routinely used for confirmation of successful surgery.

\section{Answer C}

Sestamibi scanning involves using a radioisotope, technetium-99 m, which is taken up by cells with high mitochondrial activity. It is more accurate for single adenomas than for four gland hyperplasia. Sestamibi scanning and to a lesser extent ultrasound (B) are the most frequently used imaging tests to localize the involved gland(s) in primary hyperparathyroidism. Localizing studies are generally not indicated in secondary or tertiary hyperparathyroidism, since multiplegland hyperplasia is the expected underlying pathology. Preoperative FNA (D) is not helpful in the workup of primary 
hyperparathyroidism. In about $85 \%$ of patients, imaging will localize the abnormal parathyroid gland, and a great majority will have a single parathyroid adenoma. If localizing scans are negative, yet the diagnosis of primary hyperparathyroidism is clearly established, surgery is still performed at which time intraoperative exploration of all four glands $(E)$ is performed.

\section{Answer C}

With the increasing use of routine laboratory testing, most patients with primary hyperparathyroidism are currently discovered incidentally in asymptomatic patients. Although the patients may be asymptomatic, long-standing hyperparathyroidism can lead to kidney injury and osteoporosis. Evidence of such should be sought out via bone mineral density testing as well as calculation of creatinine clearance. For patients with asymptomatic hyperparathyroidism diagnosed through laboratory screening, a 2008 consensus statement recommended the following indications for surgery:

1. Serum calcium $1.0 \mathrm{mg} / \mathrm{dL}$ greater than the upper limit of normal

2. Creatinine clearance reduced to $<60 \mathrm{~mL} / \mathrm{min}$

3. Bone mineral density with T-score less than -2.5 at any site
4. Age $<50$

5. Patients that do not desire or cannot undergo routine surveillance

The patient described meets the age criterion for surgical intervention. The surgical treatment of primary hyperparathyroidism due to four gland hyperplasia is to remove 3.5 glands. An acceptable alternative is to remove all four glands and to reimplant half of a gland within the muscles of the forearm. That way if the patient develops recurrent hyperparathyroidism, additional parathyroid tissue can be removed from the forearm under local anesthesia as opposed to reoperative neck surgery with the attendant risk of cranial nerve injury. Removal of all four glands (B) is not recommended as it will render the patient permanently hypocalcemic with a lifelong need for calcium supplementation. Observation (A) would not be appropriate for patients meeting criteria for surgery. Patients not selected for surgical therapy require biochemical monitoring of serum calcium and serum creatinine annually (D). Bone mineral density should be measured every 1-2 years. Cinacalcet (E), a calcimimetic, is mainly used to treat secondary hyperparathyroidism (seen in patients with renal failure). It may be considered to reduce the serum calcium in patients who are not candidates for surgery. 


\section{Head and Neck}

\section{Areg Grigorian and Christian de Virgilio}

\section{Questions}

1. A 65-year-old male is diagnosed with squamous cell laryngeal cancer. Examination of the neck reveals no adenopathy. At the time of biopsy, the cancer is determined to be small, and the vocal cord is still moving. It is determined that the laryngeal cancer is likely an early stage. Which of the following would be recommended next?
(A) Chest X-ray
(B) MRI
(C) PET scan
(D) Bronchoscopy
(E) No additional imaging needed

2. A 9-year-old male with a past medical history of Acute myeloid leukemia (AML) status post with bone marrow transplant presents with right ear pain and a headache. His vaccination history is not available. His mother reports that the pain started 3 days ago and is accompanied by pruritus and a sensation of fullness in the ear. His headache began suddenly and has gotten worse over the past $2 \mathrm{~h}$. Otoscopic examination reveals a green/gray discharge, an erythematous ear canal, and a normal-appearing tympanic membrane. What is the most likely organism responsible for this patient's presentation?
(A) Streptococcus pneumoniae
(B) Haemophilus influenza
(C) Moraxella catarrhalis
(D) Mycoplasma
(E) Aspergillus niger

3. A 60-year-old homeless man arrives to the ED complaining of fevers, neck pain, extreme thirst, and difficulty breathing. He has several rotted teeth extracted recently. On physical examination, his temperature is $102.7^{\circ} \mathrm{F}$, heart rate is $120 / \mathrm{min}$, respiratory rate is $24 / \mathrm{min}$, and blood pressure is $120 / 70 \mathrm{mmHg}$. He has a strong smell of alcohol on his breath. He appears agitated, and his breathing is labored. His voice sounds brassy. He is leaning forward and spitting up his saliva. On physical exam his neck is markedly swollen on the left side just below his mandible. The overlying skin is red. No fluctuance is palpated. He is unable to open his mouth. What is the best next step in management?

(A) Immediate incision and drainage at bedside

(B) Laryngoscopy

(C) Broad-spectrum antibiotics
(D) Vigorous fluid hydration

(E) Establish airway

4. A 50-year-old male smoker presents with a $2 \mathrm{~cm}$ lymph node in his left mid neck that he states has been present for 8 weeks. He denies any symptoms. Flexible nasopharyngoscopy in the office is negative. CT of the neck and chest is negative. FNA of the node confirms metastatic squamous cell carcinoma. What is the next best step in the management?

(A) Excision of lymph node

(B) Modified radical neck dissection

(C) Laryngoscopy

(D) Radiation and chemotherapy

(E) Laryngoscopy, esophagoscopy, bronchoscopy (or panendoscopy) with random biopsies

5. A 6-year-old boy presents to the ED with fevers, hearing loss, and ear pain. He finished a 10-day course of amoxicillin 1 day ago to treat an episode of acute otitis media. His ear pain initially resolved after starting antibiotics but came back 2 days ago and is now localized behind the ear. His temperature is $101.3{ }^{\circ} \mathrm{F}$, blood pressure is $110 / 82 \mathrm{mmHg}$, and pulse is $105 / \mathrm{min}$. On physical examination, his ear is superiorly displaced. What is the best next step in management?

(A) Observation with follow-up in 2 weeks

(B) Oral corticosteroids

(C) CT scan

(D) Augmentin (amoxicillin with clavulanic acid)

(E) Operating room

6. A 30-year-old Asian woman arrives for her yearly physical. During examination of her mouth, you notice a bony, immobile mass in the midline of her hard palate. She has no complaints and reports that the mass has been there for the past year. What is the best next step in management?
(A) Operative management
(B) Medical management
(C) Biopsy
(D) Observation
(E) Epstein-Barr virus-related serologic antibody test

7. A 6-year-old girl arrives to the ED by paramedics after a follow-up visit to her doctor for acute otitis media. She was witnessed having two generalized tonic-clonic seizures over $15 \mathrm{~min}$ without recovering consciousness between seizures. Her mother reports that she has had recurrent fevers, headaches, and weakness of her right arm over the past 2 weeks. She has no seizure history. She went to Mexico over the weekend to visit her family. CT scan of the head shows a single rim-enhancing lesion with a thickened capsule and diminished hypodense 
central cavity. After stabilization, what is the most appropriate next step in management?
(A) Pyrimethamine and sulfadiazine
(B) Albendazole with steroids
(C) Surgical drainage
(D) Phenytoin and valproate for at least 2 years
(E) Chemotherapy and radiation

8. A 45-year-old female presents with persistent hoarseness. Laryngoscopy reveals multiple cauliflower-like growths around her vocal cords bilaterally. Biopsy reveals an exophytic growth of keratinized squamous epithelium without malignancy. Which of the following is the most appropriate initial management?
(A) Testing for HPV and laser fulguration
(B) Testing for HIV and laser fulguration
(C) Testing for EBV and radiation therapy
(D) Laryngectomy
(E) Antiviral medication

9. A 70-year-old woman presents with complaints of mouth pain while chewing and night sweats. She has had many past episodes of sialolithiasis in her parotid gland, but they often resolve spontaneously after a few days of sucking on lemon drops. Physical examination reveals a swollen right parotid gland which is later confirmed to be a pleomorphic adenoma. She undergoes a parotidectomy. Shortly after the procedure, she complains of numbness over her right earlobe. Which nerve was most likely injured?
(A) Branch of the facial nerve
(B) Branch of the cervical plexus
(C) Branch of the trigeminal nerve
(D) Spinal accessory nerve
(E) Branch of the vagus nerve

10. A 28-year-old man presents for a routine annual physical exam. He has no significant past medical history. His temperature and vitals are stable, and his laboratory examination is benign. He smokes one pack per day. On physical examination, he has a freely moving $2 \mathrm{~cm}$ cervical lymph node. What is the best next step in management?
(A) FNA
(B) CT scan of the head and neck with contrast
(C) CT scan of the head and neck without contrast
(D) Observation and follow-up in 3 weeks
(E) Panendoscopy

11. A 4-year-old boy is brought to the ED by his parents for difficulty breathing. His mother reports that he developed nasal congestion and malaise 2 days ago, but over the past $12 \mathrm{~h}$, he has had continuous low-pitched coughs. His temperature is $101{ }^{\circ} \mathrm{F}$. On physical exam, he has pharyngeal erythema, cervical lymphadenopathy, and inspiratory stridor. Neck radiograph shows subglottic narrowing of the airway. He appears to be in respiratory distress and subsequently requires rapid sequence intubation. What is the most likely diagnosis?
(A) Respiratory distress syndrome
(B) Epiglottitis
(C) Laryngotracheobronchitis
(D) Bronchiolitis
(E) Laryngomalacia

12. Foreign body aspiration is a common occurrence in children. It can be potentially life threatening as it may obstruct the airway, preventing adequate oxygenation and ventilation. Where is the most likely location of obstruction in a patient younger than one?
(A) Right mainstem bronchus
(B) Left mainstem bronchus
(C) Upper trachea
(D) Carina (cartilaginous ridge in the lower trachea)
(E) Larynx

13. A 45-year-old male presents with a mass in his face, just below his ear. He denies any symptoms. On physical exam, the mass appears to be within the parotid gland. The mass feels firm, non-tender, and somewhat mobile. The facial nerve is intact. There is no additional mass inside the mouth. The mass most likely represents a:
(A) Pleomorphic adenomas (mixed tumor)
(B) Papillary cystadenoma (Warthin's tumor)
(C) Mucoepidermoid carcinoma
(D) Adenoid cystic carcinoma
(E) Oncocytoma

14. A 40-year-old female presents with soreness and chronic inflammation of her tongue and difficulty swallowing, stating that she feels like she is choking, particularly when eating solid foods. Laboratory examination is significant for a hemoglobin of $10.5 \mathrm{~g} / \mathrm{dL}$ (normal $12-15.2 \mathrm{~g} / \mathrm{dL})$, hematocrit of 31 (37-46\%), and MCV of $75 \mathrm{fL}$ (80-100 fL). Which of the following is the best initial test to work up this condition?
(A) Direct laryngoscopy
(B) Indirect laryngoscopy
(C) Bronchoscopy
(D) Esophagoscopy
(E) Esophagram

15. A 56-year-old male presents with chronic laryngitis that has persisted for 6 weeks. Prior to that, he acquired a cold, after which he first noticed a voice change. He also states that he is a teacher and thinks he may be wearing out his voice. He was evaluated by his primary 
care doctor and was told it was likely due to a viral illness. Past medical history is otherwise negative. He quit smoking about 5 years ago. What is the next step in the management?
(A) Five-day course of oral corticosteroids
(B) Oral antibiotics
(C) Offer reassurance and reassess in 4-6 weeks
(D) Indirect laryngoscopy in the office
(E) Direct laryngoscopy in the office

16. A 60-year-old male with poorly controlled diabetes presents to the ED with a 2-day history of fevers, nasal stuffiness, facial pain, and right retro-orbital headache.
On physical exam his temperature is $102.7^{\circ} \mathrm{F}$. The right side of his face is erythematous. There is a black eschar on his nose, as well as black discharge from his right nares. The skin on the right side of his face is numb to pin prick. Treatment consists of:

(A) Broad-spectrum antibiotics and urgent wide surgical debridement

(B) Liposomal amphotericin B and urgent wide surgical debridement

(C) Broad-spectrum antibiotics alone

(D) Liposomal amphotericin B alone

(E) Hyperbaric oxygen 


\section{Answers}

\section{Answer A}

A chest X-ray is routinely performed to rule out a concurrent primary lung cancer or pulmonary metastases. This is important as a majority of laryngeal and lung cancers are attributed to smoking. In addition, the most common location for distant metastasis of head and neck squamous cell carcinoma is the lungs. Additional imaging such as MRI (B), PET (C), or bronchoscopy (D) is not considered necessary for early stage laryngeal cancer.

\section{Answer $E$}

This patient most likely has malignant otitis externa secondary to otomycosis. Aspergillus niger is the most common cause of otomycosis and can present very similarly to otitis externa. However, patients with otomycosis will complain of an intense fullness in the ear and pruritus, and physical exam will be significant for a gray exudate from the affected ear. Unlike otitis media, patients with otomycosis will have a normal-appearing tympanic membrane as this typically affects the external ear canal. The two high-risk populations for malignant otitis externa secondary to otomycosis include patients with acute myeloid leukemia and/or diabetic ketoacidosis. Depending on the extent of local spread, patients can present with a myriad of symptoms including blindness, headache, seizure, and coma. CT scan of the head will help evaluate the extent of damage and infiltration and help guide surgical management (e.g., debridement, washout). Answer choices A-C are all common causes of otitis media with Streptococcus pneumoniae being the most common organism. Mycoplasma (D) has been associated with bullous myringitis, which is characterized by vesicular inflammation of the tympanic membrane and is seen most commonly with untreated otitis media. Patients will present with very tender ear canals, and otoscopy shows large red vesicles on the tympanic membrane.

\section{Answer $\mathrm{E}$}

Ludwig's angina is characterized by a progressive cellulitis in the floor of the mouth and often involves the submandibular space (which is divided by the mylohyoid muscle). It can present with fevers, neck pain, neck swelling, dental pain, dysphagia, and drooling. This can be life threatening as it can lead to airway obstruction. The majority of cases follow dental procedures which allow bacteria from a tooth infection to migrate into the submandibular space. Patients with labored breathing and marked swelling require an immediate airway. This may be achieved via endotracheal intubation or alternatively via a surgical airway (cricothyroidotomy or tracheostomy). The neck infection will then need immediate surgical drainage, (A) but this is best accomplished in the operating room. Broad-spectrum antibiotics (C) and IV fluids (D) are also necessary, but should not be prioritized over the airway.
Laryngoscopy (B) is not recommended as it will only delay establishment of the airway and any potential trauma/gagging may further compromise the airway.

\section{Answer $\mathrm{E}$}

A solitary enlarged lymph node that persists beyond 3 weeks particularly in a middle-aged male smoker should be considered a metastatic lymph node until proven otherwise. Oftentimes, the patient will have symptoms (such as hoarseness, persistent sore throat, ulcerative lesions) that will guide the workup. But if no symptoms are present, a flexible nasopharyngoscopy is used initially to evaluate the nasal cavities, nasopharynx, oropharynx, hypopharynx, and glottis to look for a site of primary tumor. FNA is subsequently performed for to confirm that the solitary neck mass is a metastatic lymph node. Once FNA confirms this, CT scan of the neck may identify the primary. If the primary is still not evident, the next step is to try to identify the location of the primary tumor using a panendoscopy (also termed triple endoscopy) with random biopsies. This involves a complete endoscopic evaluation of the upper aerodigestive track, including laryngoscopy (C), esophagoscopy, and bronchoscopy under general anesthesia in the operating room. A neck dissection (B) would not be considered until after panendoscopy. Radiation and chemotherapy (D) may be used as adjuncts depending on the stage and grade of the primary tumor.

\section{Answer C}

Mastoiditis usually occurs days to weeks after an episode of acute otitis media. Patients present with fevers and complaints of a red, swollen, and tender area behind the ear (mastoid process). Physical exam may reveal a displaced ear on the affected side. The diagnosis can be confirmed with a CT scan of the mastoid process and is recommended for patients suspected of having mastoiditis. Patients with CT-confirmed acute surgical mastoiditis are candidates for mastoidectomy with insertion of a tympanostomy tube (E). Observation (A) is not an appropriate management for patients with mastoiditis. Oral corticosteroids (B) are not considered part of the management of acute mastoiditis. Augmentin (D) would be an appropriate choice for patients with acute otitis media suspected of having a resistant strain.

\section{Answer D}

This patient most likely has torus palatinus, a bony benign mass located on the hard palate of the mouth. The cause is unknown. It occurs more frequently in women and those of Asian descent. There is no associated malignant transformation. Biopsy $(\mathrm{C})$ is not warranted and patients only need reassurance. Operative management (A) with surgical removal would be indicated only for symptomatic patients (e.g., interference with denture placements, pain, trouble swallowing). There is no medical management (B) available 
for torus palatinus. Nasopharyngeal carcinoma, a rare tumor arising from the epithelium of the nasopharynx, occurs more frequently in patients of Asian descent and those infected with EBV (E). However, a bony outgrowth of the hard palate would not be expected in these patients.

\section{Answer C}

Although it occurs infrequently, brain abscesses are a complication of acute otitis media. It shows many of same manifestations as a brain tumor (space occupying) but with a much shorter timetable (week or two). Patients typically have a fever, acute onset of headache, focal neurologic findings (e.g., weakness in the right arm), seizure, and an obvious source, such as otitis media or mastoiditis. A MRI or CT of the head will find ring enhancing lesions. Treatment is open drainage (by a neurosurgeon). All these patients should also be started on empiric antibiotics. Although controversial, some clinicians also administer corticosteroids as it may have some benefit in decreasing the growth of the abscess and preventing cerebral edema. Ring-enhancing lesions and seizures can also be found in patients with CNS lymphoma, toxoplasmosis, or neurocysticercosis. CNS lymphoma primarily occurs in patients with AIDS. Pyrimethamine and sulfadiazine (A) would be the appropriate choice to treat patients with toxoplasmosis. Seizures secondary to neurocysticercosis (Taenia solium) can occur in patients with a recent travel history to Mexico, but this patient's temporal relation of her acute condition and a recent episode of otitis media make this less likely. Patients with neurocysticercosis should be started on antiparasitics, such as albendazole, and corticosteroids (B). Antiepileptics (D) can be used to manage her acute condition, but it is unlikely that she also has a concurrent seizure disorder obviating the need for long-term antiepileptic therapy. Chemotherapy and radiation (E) would be considered in patients with brain malignancies.

\section{Answer A}

Laryngeal papillomas or recurrent respiratory papillomatosis is a condition caused by human papilloma virus (HPV) types 6 and 11. Infection with the virus can lead to benign papillary tumors of the larynx (cauliflower-like growths) and presents primarily with hoarseness. It rarely gives rise to laryngeal carcinoma. Laser fulguration can be performed to destroy the papillary growths. Laryngectomy (D) would not be appropriate. HIV (B) can present with various AIDSdefining malignancies including Kaposi's sarcoma, nonHodgkin's lymphoma, and cervical cancer. EBV (C) has been associated with nasopharyngeal carcinoma and Burkitt's lymphoma. Antiviral agents (e.g., cidofovir) are used as an adjunct to laser ablation to prevent recurrence, but they are not the primary treatment modality.

\section{Answer B}

Sialolithiasis (salivary ductal stones) can increase the risk of developing a tumor of the gland. Lemon drops will stimulate saliva production and help facilitate passage of the stone. Pleomorphic adenoma is benign and considered the most common neoplasm of the parotid gland. The greater auricular nerve is a branch of the cervical plexus (C2-C3) and provides cutaneous sensation to the lower portion of the ear, including the earlobe. The facial nerve $(\mathrm{A})$, which traverses through the two lobes of the parotid gland, can also be injured and will present with facial droop. Injury to the trigeminal nerve (C) can cause widespread numbness in the face. However, this type of injury occurs rarely because of the deep location, immediate branching, and redundancy of these nerves. An injured spinal accessory nerve (D) will present with partial paralysis of the trapezius and sternocleidomastoid muscles. The auricular branch of the vagus nerve (E) provides cutaneous sensation to the ear canal, not the earlobe.

\section{Answer D}

The most appropriate recommendation for a young patient presenting with a newly discovered, isolated, and enlarged cervical node is observation with follow-up and reexamination in 3 weeks. If the node disappears, it most likely was inflammatory in nature. However, if this patient presented with any red-flag symptoms (e.g., dysphagia, odynophagia, dysphonia, hoarseness, and weight loss), additional workup would be required to rule out malignancy. CT scan $(B, C)$ with contrast is the initial preferred imaging modality for a solitary neck mass that is concerning for malignancy (following a careful head and neck examination). FNA (A) is indicated for neck masses that are persistent, enlarging, or suspicious for malignancy. Panendoscopy (E) is performed in the operating room in the setting of a metastatic neck lymph node (when the primary is occult).

\section{Answer $C$}

Croup, also known as laryngotracheobronchitis, is caused by the parainfluenza virus and primarily affects young children. The cough associated with this condition is described as a low-pitched seal-like bark. The diagnosis can be confirmed by looking for the classic "steeple sign" on posteroanterior $\mathrm{X}$-ray of the neck, which is indicative of subglottic narrowing. Patients are at risk for airway obstruction and will require intubation if they appear to be in respiratory distress. Management includes steroids and aerosolized racemic epinephrine. Respiratory distress syndrome of the newborn (A) is caused by surfactant deficiency. It occurs within 2 days of birth and presents with cyanosis, nasal flaring, crackles, and expiratory grunting. Epiglottitis $(\mathrm{C})$ is a rapidly progressive 
infection of the epiglottis, most commonly due to Haemophilus influenzae type $B$. Patients with epiglottitis may require intubation or even tracheostomy due to airway compromise from the swollen epiglottis. Bronchiolitis (D) is characterized by a viral infection of the bronchioles and occurs most commonly in patients $<2$ years old. Laryngomalacia (E) is a congenital abnormality of the laryngeal cartilage and can result in collapse of the supraglottic structures in newborns, leading to airway obstruction. Infants with laryngomalacia should be fed upright and remain in this position for at least $30 \mathrm{~min}$ after each feed.

\section{Answer E}

The larynx is the most common site for foreign body aspiration in children younger than 1 , while the trachea $(C, D)$ and right mainstem bronchus (A) are the most common sites in older children. The left mainstem bronchus (B) is a less frequent site for foreign body aspiration owing to its acute angle as it enters the lung versus an obtuse angle in the right. Patients with foreign body aspiration may have wheezing, but using a bronchodilator increases the risk of further pushing the foreign body down the airway. Order a chest X-ray if there is a suspicion for a foreign body obstruction. Bronchoscopy is recommended for definitive diagnosis. Extracting the foreign body requires a rigid bronchoscopy.

\section{Answer A}

Most salivary gland tumors are in the parotid gland, and the majority are benign $(80 \%)$. The most common type of parotid gland tumor is a pleomorphic adenoma. Although benign, it does have a known risk of malignant transformation that becomes as high as 10-25\% when present beyond 15 years. Warthin's tumor (B) is the second most common benign salivary tumor and is strongly related to smoking. Mucoepidermoid carcinoma $(\mathrm{C})$ is the most common malignant salivary gland tumor. Facial nerve involvement is more suggestive of malignant transformation. The second most common malignancy is adenoid cystic carcinoma (D). Oncocytoma $(\mathrm{E})$ is a rare $(1-2 \%)$ salivary gland tumor and most often involves the parotid gland.

\section{Answer $E$}

The triad of dysphagia, esophageal webs (e.g., feeling of choking with solid foods), and iron-deficiency anemia is highly suggestive of Plummer-Vinson syndrome. The pathophysiology still remains unclear but is most likely multifactorial. Barium esophagram is one of the most sensitive methods and diagnostic tests of choice to confirm the presence of esophageal webs, which appears as a thin projection off the postcricoid, anterior esophageal wall. If esophagram is equivocal, esophagoscopy (D) can be used next. Laryngoscopy (A-B) or bronchoscopy (C) is not typically required in the workup for Plummer-Vinson syndrome. However, if there is any concern for head and neck cancer (e.g., neck mass in patient with smoking history), a panendoscopy can be considered in the workup.

\section{Answer D}

In an older (>50) male patient with a history of smoking, presenting with persistent laryngitis and recent difficulty in projecting his voice, laryngeal cancer must be ruled out. The initial test is to evaluate the larynx and vocal cords with indirect laryngoscopy in the office (with administration of local anesthetic spray to the back of the throat). It is termed indirect, as it has a mirror that permits indirect visualization of the vocal cords. Structural abnormalities, such as masses, ulcers, or mucosal irregularities, may be noted, as well as motion of the vocal cords. Direct laryngoscopy (E) is done in the OR under general anesthesia. It involves insertion of a rigid metal tube directly into the larynx and allows for biopsies to be taken. Given the high likelihood of cancer, antibiotics (B) or reassurance (C) would be inappropriate.

\section{Answer B}

This patient's presentation is concerning for mucormycosis, most commonly caused by Rhizopus or Mucor fungi. Patients with poorly controlled diabetes and/or neutropenia are the most common groups affected with mucormycosis. They most often present with local invasion of the fungi into the facial sinuses and eventually the brain (e.g., sudden onset or worsening of headache), as in this patient. Black eschar on the nose and discharge from the nares is characteristic of mucormycosis. Management consists of immediate antifungal therapy with liposomal amphotericin B and surgical debridement. Antifungal therapy alone would be inappropriate, as well as antibiotics (A,C-D). Hyperbaric oxygen (E) is currently being investigated as an adjunctive therapy for select patients with mucormycosis. Mortality for mucormycosis ranges from $50 \%$ to $75 \%$. 


\section{Hepatopancreaticobiliary}

\section{Areg Grigorian, Paul N. Frank, Danielle M. Hari, and} Christian de Virgilio

\section{Questions}

1. A 55-year-old male arrives to the ED with $40 \%$ total body surface area second and third degree burns over his arms and legs after hot tar spilled on him at a jobsite where he was working as a roofer. He is in critical condition and intubated in the ICU. On the fifth hospital day, he spikes a temperature of $102{ }^{\circ} \mathrm{F}$, blood pressure is $110 / 80 \mathrm{mmHg}$, and pulse is $92 / \mathrm{min}$. On physical exam he has tenderness on palpation of the RUQ and epigastrium, absent bowel sounds, and multiple healing burn wounds that appear to be clean. Laboratory exam demonstrates a WBC of $16 \times 10^{3} / \mu \mathrm{L}$ (normal $4.1-10.9 \times 10^{3} / \mu \mathrm{L}$ ) with $12 \%$ bands, amylase of $180 \mu / \mathrm{L}$ (normal $30-110 \mu / \mathrm{L}$ ), lipase of $55 \mu / \mathrm{L}(7-60 \mu / \mathrm{L})$, alkaline phosphatase of $70 \mu / \mathrm{L}(33-131 \mu / \mathrm{L})$, and total bilirubin of $1.2(0.1-$ $1.2 \mathrm{mg} / \mathrm{dl}$ ). An abdominal X-ray series reveals distended loops of small bowel and large bowel without air fluid levels and no free air under the diaphragm. An abdominal ultrasound demonstrates a distended gallbladder with pericholecystic fluid and no stones. What is the most likely underlying etiology?
(A) Acute pancreatitis
(B) Cholecystitis
(C) Cholangiohepatitis
(D) Perforated duodenal ulcer
(E) Acute cholangitis

2. A 55-year-old woman is evaluated in the emergency department for a 2-day history of severe epigastric abdominal pain, nausea, and vomiting. In reviewing her past medical history, she states she was evaluated 6 months ago for mild, but similar intermittent abdominal pain and was lost to follow-up. She does not take any medications. She has 1-2 drinks of alcohol on social occasions. On physical examination, temperature is $99.2^{\circ} \mathrm{F}$, blood pressure is $132 / 82 \mathrm{mmHg}$, pulse is $101 / \mathrm{min}$, and respirations are 20/min. There is epigastric tenderness and RUQ tenderness. Labs are drawn and shown below. What is the most likely diagnosis?

AST: $523 \mu / \mathrm{L}$ (normal $5-35 \mu / \mathrm{L}$ )

ALT: $622 \mu / \mathrm{L}(7-56 \mu / \mathrm{L})$

TBilli: $2.0 \mathrm{mg} / \mathrm{dL}(0.1-1.2 \mathrm{mg} / \mathrm{dL})$

Alkaline phosphatase: $450 \mu / \mathrm{L}(33-131 \mu / \mathrm{L})$

Amylase: $1300 \mu / \mathrm{L}(30-110 \mu / \mathrm{L})$

Lipase: $1000 \mu / \mathrm{L}(7-60 \mu / \mathrm{L})$

(A) Acute pancreatitis secondary to alcohol

(B) Acute pancreatitis secondary to gallstones
(C) Acute pancreatitis secondary to hypertriglyceridemia

(D) Acute pancreatitis secondary to hypercalcemia

(E) Chronic pancreatitis

3. A 60-year-old man presents with yellowing of his skin. He reports that he has unintentionally lost $10 \mathrm{lb}$ over the last 5 months. He denies abdominal pain or fevers. He has also developed pruritus, dark urine, and clay-colored stools. He has smoked two packs per day for the past 40 years. On physical exam, his temperature is $98.6^{\circ} \mathrm{F}$, blood pressure is $110 / 86 \mathrm{mmHg}$, and pulse is $94 / \mathrm{min}$. On physical examination, he appears jaundiced and has scleral icterus. He has fullness, suggestive of a mass in his RUQ that is not tender to palpation. What is the best term to describe this constellation of findings?
(A) Cullen's sign
(B) Charcot's triad
(C) Reynold's pentad
(D) Courvoisier's sign
(E) Murphy's sign

4. A 46-year-old man is admitted to the hospital for severe epigastric pain of 12-h duration, nausea, two episodes of vomiting, and anorexia. His past medical history is significant for alcoholism and several admissions for alcohol withdrawal. On physical exam temperature is $99.6{ }^{\circ} \mathrm{F}$, blood pressure is $137 / 84 \mathrm{mmHg}$, pulse is $99 /$ min, and respirations are $16 / \mathrm{min}$. There is moderate tenderness in the epigastrium to palpation, but the abdomen is soft and no masses are felt. There is no scleral icterus and no jaundice of the skin. Laboratory examination is shown below. What is the next step in management?

AST: $123 \mu / \mathrm{L}$ (normal $5-35 \mu / \mathrm{L}$ )

ALT: $99 \mu / \mathrm{L}(7-56 \mu / \mathrm{L})$

TBilli: $0.7 \mathrm{mg} / \mathrm{dL}(0.1-1.2 \mathrm{mg} / \mathrm{dL})$

Lipase: $709 \mu / \mathrm{L}(7-60 \mu / \mathrm{L})$

Alkaline phosphatase: $709 \mu / \mathrm{L}(33-131 \mu / \mathrm{L})$

WBC: $11 \times 10^{3} / \mu \mathrm{L}$ (normal $4.1-10.9 \times 10^{3} / \mu \mathrm{L}$ )

Hgb: $12.9 \mathrm{mg} / \mathrm{dL}(13.2-16.2 \mathrm{mg} / \mathrm{dL})$

(A) Start intravenous antibiotics

(B) CT scan

(C) ERCP with sphincterotomy

(D) NPO, IV hydration, and analgesics

(E) Chlordiazepoxide (Librium) for alcohol withdrawal

5. A 45-year-old healthy woman arrives for follow-up after her primary care physician discovered gallstones incidentally while performing imaging studies for an unrelated event. She has no complaints and has a healthy diet but is worried about the stones. An abdominal ultrasound is repeated and demonstrates several stones in her gallbladder without any wall thickening. What is the recommended management for this patient? 

(A) Prophylactic cholecystectomy
(B) Ursodeoxycholic acid
(C) Endoscopic retrograde cholangiography (ERCP)
(D) Observation
(E) Extracorporeal shock wave lithotripsy

6. A 30-year-old man is admitted to the hospital for severe acute pancreatitis due to alcohol abuse. His hospital course is complicated by transient renal insufficiency. On hospital day 20, the patient complains of increasing epigastric abdominal pain, nausea, and vomiting. On physical examination, he has a fever of $102^{\circ} \mathrm{F}$ and a heart rate of $110 / \mathrm{min}$. Abdominal examination reveals marked epigastric tenderness. His lungs are clear bilaterally. WBC count is $14.5 \times 10_{3} / \mu \mathrm{L}$ (normal $4.1-10.9 \times 10_{3} / \mu \mathrm{L}$ ) with $10 \%$ bands. Blood cultures are sent, and fluid bolus is given. What is the next step in management?

(A) Start intravenous antibiotics

(B) CT scan of the abdomen with contrast

(C) ERCP with sphincterotomy

(D) Exploratory laparotomy for pancreatic debridement

(E) Laparoscopy

7. A 65-year-old man presents to the ED with RUQ pain. $\mathrm{He}$ is diagnosed with acute cholecystitis and undergoes a cholecystectomy the following day. He is discharged shortly after his procedure. Five days later, he arrives back to the ED with abdominal pain and low-grade fevers. On physical examination, his blood pressure is $120 / 70 \mathrm{mmHg}$, heart rate is $90 / \mathrm{min}$, and temperature is $99.0^{\circ} \mathrm{F}$. He has diffuse mild abdominal tenderness to palpation. His laboratory examination is significant for white blood count of $16.9 \times 10^{3} / \mu \mathrm{L}$ (normal 4.1 $\left.10.9 \times 10^{3} / \mu \mathrm{L}\right)$. What is the next best step?
A) Endoscopic ultrasound
(B) Exploratory laparotomy
(C) CT scan of abdomen
(D) ERCP with stenting
(E) HIDA scan

8. A 63-year-old man is admitted to the hospital for alcohol pancreatitis. At $48 \mathrm{~h}$ after admission, he manifested four of Ranson's criteria. On hospital day 6 he is reevaluated on rounds for increasing epigastric abdominal pain. He denies any vomiting. On physical exam temperature is $100.3^{\circ} \mathrm{F}$, blood pressure is $134 / 74 \mathrm{mmHg}$, pulse is $89 / \mathrm{min}$, and respirations are $16 / \mathrm{min}$. The belly is distended but soft, and there is still significant epigastric tenderness. CT scan is obtained and shows diffuse edema surrounding the pancreas with a pancreatic phlegmon, but no evidence of necrosis. What is most appropriate approach to his nutritional management?
(A) Continue NPO and intravenous normal saline

(B) Clear liquid diet

(C) Enteral nutrition via feeding tube

(D) Parenteral nutrition via central line

(E) Parenteral nutrition via peripheral line

9. A 52-year-old insulin-dependent diabetic man is evaluated for vague epigastric pain, is diagnosed with GERD, and is treated with proton pump inhibitors with resolution of symptoms. In the course of the workup, however, an abdominal ultrasound was performed. No gallstones were seen, but an incidental $12 \mathrm{~mm}$ polyp was found within the gallbladder. What is the next best step in management?
(A) Laparoscopic cholecystectomy
(B) Open cholecystectomy
(C) Percutaneous gallbladder drainage
(D) Endoscopic ultrasound
(E) Repeat ultrasound in 6 months

10. A 41-year-old man with alcoholism is admitted to the ICU with a diagnosis of acute pancreatitis. He has three Ranson's criteria on admission and two more at $48 \mathrm{~h}$. He requires aggressive fluid resuscitation to maintain his blood pressure in the first $24 \mathrm{~h}$, but over the next 3 days, his blood pressure stabilizes. On the third day of admission, he develops tachypnea, tachycardia, and hypoxia with oxygen saturation to $89 \%$. Central venous pressure is $8 \mathrm{mmHg}$. The patient is placed on nasal cannula, but the oxygen saturation remains the same. His temperature is $98.9^{\circ} \mathrm{F}$, pulse is $104 / \mathrm{min}$, and blood pressure is $129 / 73 \mathrm{mmHg}$. A chest X-ray is obtained and shows bilateral infiltrates. Labs are drawn and shown below. What is the most likely diagnosis?

AST: $75 \mu / \mathrm{L}$ (normal $5-35 \mu / \mathrm{L}$ )

ALT: $92 \mu / \mathrm{L}(7-56 \mu / \mathrm{L})$

WBC: $11 \times 10^{3} / \mu \mathrm{L}$ (normal $4.1-10.9 \times 10^{3} / \mu \mathrm{L}$ )

Arterial blood gas: $\mathrm{pH} 7.44, \mathrm{PaO}_{2} 66 \mathrm{mmHg}, \mathrm{PaCO}_{2}$ $36 \mathrm{mmHg}$

(A) Adult respiratory distress syndrome (ARDS)

(B) Pulmonary embolism

(C) Hospital-acquired pneumonia

(D) Fluid overload (pulmonary edema)

(E) Atelectasis

11. Which laboratory finding is consistent with obstructive jaundice?

(A) Increased urine urobilinogen

(B) Increased urine conjugated bilirubin

(C) Increased stool stercobilin

(D) Indirect > direct hyperbilirubinemia

(E) Elevation of transaminases out of proportion to alkaline phosphatase 
12. Which of the following is a risk factor for pancreatic cancer?
(A) Alcohol
(B) Smoking
(C) Prostate cancer in the family
(D) Malabsorption
(E) Pancreatic enzyme supplementation

13. A 60-year-old woman arrives to the emergency department with bloody emesis. She has a past medical history significant for hypertension and an episode of severe pancreatitis due to alcohol abuse 1 year ago and has since developed chronic pancreatitis. Her temperature is $98.6^{\circ} \mathrm{F}$, blood pressure $110 / 88 \mathrm{mmHg}$, and pulse of $88 /$ min. Esophagogastroduodenoscopy shows bleeding coming from isolated gastric varices. Which of the following is most likely to successfully treat the bleeding?
(A) Liver transplantation
(B) Endoscopic banding of the varices
(C) Endoscopic sclerotherapy
(D) TIPS (transjugular portosystemic shunt)
(E) Splenectomy

14. A 56-year-old male undergoes a Whipple procedure for pancreatic adenocarcinoma. Two days later, there is about $30 \mathrm{~cm}^{3}$ of white/opal opaque drainage emanating from the patient's drain. What is the most appropriate next step?
(A) Obtain abdominal CT scan
(B) Obtain abdominal ultrasound
(C) Send fluid for amylase level
(D) Start octreotide
(E) Initiate total parenteral nutrition (TPN)

15. Which of the following is an appropriate use of CA 19-9?

(A) Screening normal, healthy patients for pancreatic cancer

(B) Screening at-risk patients for pancreatic cancer

(C) Confirming diagnosis of pancreatic cancer in patients with periampullary mass on $\mathrm{CT}$

(D) Monitor for progression of disease following resection and/or adjuvant therapy

(E) None of the above

16. A 45-year-old presents with a 1 day history of RUQ pain and tenderness, nausea, and vomiting. Physical examination is significant for marked RUQ tenderness and guarding. Laboratory values are significant for a WBC of $12 \times 10^{3} / \mu \mathrm{L}$ (normal $4.1-10.9 \times 10^{3} / \mu \mathrm{L}$ ) with $10 \%$ bands, total bilirubin of $1.2 \mathrm{mg} / \mathrm{dL}(0.1-1.2 \mathrm{mg} / \mathrm{dL})$, AST of $110 \mu / \mathrm{L}$ (normal 5-35 $\mu / \mathrm{L}$ ), ALT of $120 \mu / \mathrm{L}(7-56 \mu / \mathrm{L})$, and alkaline phosphatase of $90 \mu / \mathrm{L}(33-131 \mu / \mathrm{L})$. RUQ ultrasound reveals several gallstones, a thickened gallbladder wall, and a normal common bile duct. Optimal management consists of:

(A) Schedule for elective outpatient laparoscopic cholecystectomy

(B) Admit, IV antibiotics, laparoscopic cholecystectomy within $48 \mathrm{~h}$ of admission

(C) Admit, IV antibiotics for 4-5 days followed by laparoscopic cholecystectomy

(D) Admit, IV antibiotics until WBC normalizes, followed by outpatient laparoscopic cholecystectomy

(E) Admit, IV antibiotics, ERCP, followed by laparoscopic cholecystectomy

17. A 40-year-old female presents with moderate epigastric abdominal pain. She has a history of intermittent RUQ pain after eating fatty foods. On physical examination she is afebrile with a heart rate of $100 / \mathrm{min}$ and blood pressure of 110/70 mmHg. She has moderate epigastric tenderness to palpation. Laboratory values are significant for a WBC of $11 \times 10^{3} / \mu \mathrm{L}$ (normal $4.1-10.9 \times 10^{3} / \mu \mathrm{L}$ ) with $3 \%$ bands, total bilirubin of $1.2 \mathrm{mg} / \mathrm{dL}(0.1-$ $1.2 \mathrm{mg} / \mathrm{dL})$, AST of $250 \mu / \mathrm{L}(5-35 \mu / \mathrm{L})$, ALT of $300 \mu / \mathrm{L}$ $(7-56 \mu / \mathrm{L})$, alkaline phosphatase of $150 \mu / \mathrm{L}(33-$ $131 \mu / \mathrm{L})$, amylase of $1,300 \mu / \mathrm{L}(30-110 \mu / \mathrm{L})$, and lipase of $1,100 \mu / \mathrm{L}(7-60 \mu / \mathrm{L})$. RUQ ultrasound shows numerous small gallstones, normal gallbladder wall, and a normal common bile duct diameter of $0.4 \mathrm{~mm}$. On the second hospital day, her pain has resolved; she is afebrile and has a normal heart rate, and her WBC count has normalized. The amylase has decreased to $350 \mu / \mathrm{L}$. Optimal management consists of:

(A) Proceed with laparoscopic cholecystectomy (LC) with intraoperative cholangiogram (IOC)

(B) Wait 4-5 more days until amylase has completely normalized, and then proceed to $\mathrm{LC}$ with IOC

(C) ERCP followed by LC during same hospitalization

(D) Schedule for elective outpatient LC with IOC

(E) ERCP only

18. A 50-year-old diabetic male presents with severe RUQ pain and fevers. On physical examination, his temperature is $103.5^{\circ} \mathrm{F}$, BP is $100 / 60 \mathrm{mmHg}$, and heart rate is $120 / \mathrm{min}$. He has severe tenderness to palpation in the RUQ. WBC is $20 \times 10^{3} / \mu \mathrm{L}$ (normal $4.1-10.9 \times 10^{3} / \mu \mathrm{L}$ ) with $10 \%$ bands, total bilirubin is $1.0 \mathrm{mg} / \mathrm{dL}(0.1-$ $1.2 \mathrm{mg} / \mathrm{dL})$, amylase is $90 \mu / \mathrm{L}(30-110 \mu / \mathrm{L})$, alkaline phosphatase is $90 \mu / \mathrm{L}(33-131 \mu / \mathrm{L})$, AST is $110 \mu / \mathrm{L}$ (normal $5-35 \mu / \mathrm{L}$ ), and ALT is $140 \mu / \mathrm{L}(7-56 \mu / \mathrm{L})$. RUQ ultrasound shows gallstones, a normal common 
bile duct diameter, and a few gas bubbles within the wall of the gallbladder. IV fluids and antibiotics are administered. The next step in the management consists of:
(A) Immediate cholecystectomy
(B) Admit to ICU for $24-48 \mathrm{~h}$ of IV antibiotics and careful monitoring
(C) Cholecystostomy
(D) CT scan of abdomen
(E) ERCP

19. A 58-year-old female underwent a CT scan of her abdomen and pelvis following a motor vehicle collision
1 month ago. She has no intra-abdominal injuries and was discharged from the ED. However, her gallbladder was incidentally noted to be heavily calcified. She is otherwise in good health and denies any abdominal pain. Her past history is significant for mild hypertension. Which of the following is the best recommendation?
(A) Reassure patient that no follow-up is needed
(B) Repeat CT scan in 1 year
(C) Laparoscopic cholecystectomy
(D) Check serum calcium and PTH levels
(E) Obtain ERCP with brushings 


\section{Answers}

\section{Answer B}

Acalculous cholecystitis is a condition seen in patients that are critically ill such as those with multiorgan trauma, burns, or recent major surgery. The exact mechanism is unclear, but it is thought to be secondary to a combination of biliary stasis (from being NPO) and gallbladder ischemia as a result of hypovolemic and/or septic shock. The diagnosis can be difficult for several reasons. Patients are critically ill so a history may be unobtainable and physical exam may be unreliable. The imaging test of choice is ultrasound (US). Findings suggestive of acalculous cholecystitis include gallbladder wall thickening and pericholecystic fluid; however, such findings are not consistent. If US is not definitive, HIDA scan is the next test and is considered positive if the gallbladder is not visualized. However, false positives are seen in patients who have been NPO for a prolonged period (which many of these critically ill patients have). Gallstones are not implicated in this condition, and will not be seen on ultrasonography. Treatment of acalculous cholecystitis includes broad-spectrum antibiotics followed by urgent percutaneous cholecystostomy (if the patient is critically ill) or cholecystectomy (laparoscopic vs. open cholecystectomy) if the patient is stable enough to undergo general anesthesia. Acute pancreatitis (A) is in the differential; however, the patient's lipase is normal (more specific than amylase), and the amylase is only mildly elevated (acute pancreatitis requires an elevation 3x above normal). Mild hyperamylasemia can be seen with many intra-abdominal conditions including cholecystitis or bowel ischemia. Burn victims are at risk for stress-related mucosal damage (Curling ulcer) secondary to an inability to maintain the integrity of the gastrointestinal mucosal barrier. This may subsequently lead to perforated viscus (D) which will present with an acute abdomen and a plain film demonstrating free air under the diaphragm. Cholangiohepatitis (C) is associated with biliary parasites such as Clonorchis sinensis and is characterized by brown pigment stones that result from biliary sludge and dead bacterial cell bodies. Acute cholangitis (E) would present with evidence of cholestasis (jaundice and/or elevated liver enzymes) and biliary obstruction (dilated bile ducts on ultrasound).

\section{Answer B}

The most likely diagnosis is acute pancreatitis secondary to gallstones. More than half of all cases of pancreatitis are associated with either gallstones or alcohol. Patients with gallstone pancreatitis have extremely high serum amylase (sometimes in the thousands) and ALT (greater than $3 x$ the upper limit of normal) as compared to other etiologies. A biliary etiology of pancreatitis is further supported by the elevated bilirubin and alkaline phosphatase which suggest at least a temporary obstruction of the common bile duct by a gallstone. Most gallstones only transiently obstruct the common bile duct and pass on their own. Pancreatitis due to alcohol is seen in patients with long-standing heavy alcohol abuse (which is not suggested by the history in this patient), and not following a onetime binge. Chronic pancreatitis is rare with gallstones. It is most often seen in association with long-standing alcohol abuse. Patients present with chronic epigastric pain, steatorrhea, and/or diabetes. Amylase and lipase levels are often not elevated.

\section{Answer D}

The patient is presenting with painless jaundice, which should be considered as due to malignancy until proven otherwise. Courvoisier's sign is a term used to describe a palpable non-tender gallbladder that markedly distends as a result of a gradual common bile duct obstruction, most often by a pancreatic adenocarcinoma at the head of the pancreas (distal common bile duct and ampullary cancer are also in the differential). Gallstones typically cause sudden obstruction of the biliary tree and often harbor bacteria. They are almost always associated with pain and often trigger an inflammatory response. Charcot's triad (B) (RUQ pain, fever, and jaundice) and Reynold's pentad (C) (the triad plus hypotension and altered mental status) are associated with acute cholangitis most often secondary to a gallstone obstructing the distal common bile duct. Murphy's sign (E) (RUQ tenderness on palpation that stops inspiration) is associated with acute cholecystitis due to a gallstone obstructing the cystic duct. Cullen's sign (A) is a blue-red discoloration at the umbilicus, and the appearance is a result of digested blood products in the retroperitoneum, forming methemalbumin, that then travel towards the anterior abdominal wall. It is associated with retroperitoneal bleeding, as seen with hemorrhagic pancreatitis.

\section{Answer D}

The presentation is most consistent with pancreatitis secondary to alcohol. Lipase is more specific for pancreatitis than amylase. The vast majority of acute pancreatitis cases are due to peripancreatic inflammation, not infection. Antibiotics (A) are therefore not beneficial. CT scan (B) is not necessary to establish the diagnosis and should be reserved for situations where the diagnosis is in question or if the patient clinically deteriorates during the subsequent hospitalization. The most appropriate management is to make the patient NPO, aggressively hydrate, and administer analgesics. Routine use of an NG tube is unnecessary. ERCP (C) is utilized in gallstone pancreatitis, specifically if the patient demonstrates evidence of concomitant cholangitis or obstructive jaundice. Librium (E) may be utilized for alcohol withdrawal, but should not be prioritized ahead of aggressive hydration. The majority of patients resolve the episode of pancreatitis within 3-5 days using conservative management. 


\section{Answer D}

Gallstones are commonly discovered incidentally in asymptomatic patients after imaging studies that are performed for unrelated reasons. Only 2-3\% of asymptomatic patients develop biliary colic per year, and only a small fraction of those patients progress to complications from gallstones (such as acute cholecystitis, pancreatitis, acute cholangitis). For this reason, the vast majority of asymptomatic gallstones should not receive prophylactic cholecystectomy (A). Prophylactic cholecystectomy might be considered in patients who are planning extended travel to areas without healthcare access (Antarctica in the winter). Because patients with diabetes are at greater risk of developing complications from gallstones, some authors have recommended prophylactic cholecystectomy in diabetics. However, even in the diabetic population, it is reasonable to wait to see if symptoms develop. Ursodeoxycholic acid (B) can dissolve gallstones. However, it is completely successful in only about $1 / 3$ of cases, only for cholesterol stones, is associated with side effects (diarrhea), and is costly. In addition, the stones may recur once the medication is stopped. ERCP (C) is an invasive procedure utilized for choledocholithiasis and acute cholangitis. Extracorporeal shock wave lithotripsy (E) is effective in breaking stones into small particles, but does not prevent stone recurrence.

\section{Answer A}

The patient is manifesting evidence of systemic inflammatory response syndrome (SIRS); the presentation is most concerning for a pancreatic abscess. When SIRS is diagnosed, the first steps include fluid administration, blood cultures, and prompt institution of intravenous antibiotics (within $1 \mathrm{~h}$ ), preferably imipenem. This should be followed by a CT scan (B) with contrast looking for necrotic tissue (non-enhancing areas) and a possible pancreatic abscess. In the past, such a finding on CT would warrant immediate exploration for pancreatic debridement (D). However the current approach is termed a "step-up" approach, which consists of a series of increasingly more invasive interventions. This begins with a percutaneous attempt at drainage of any infected pancreatic collections. If that fails, the next step is laparoscopic drainage (E). Finally open surgical pancreatic debridement is recommended. $\mathrm{ERCP}(\mathrm{C})$ would be indicated for suspected acute cholangitis, usually in association with gallstones.

\section{Answer $\mathrm{C}$}

Persistent abdominal pain, fevers, and nausea beyond a few days following laparoscopic cholecystectomy should raise suspicion of a bile duct injury or a bile leak from the cystic duct stump (due to the surgical clip inadvertently coming off). Imaging by CT scan should be obtained to look for a fluid collection. Abdominal ultrasound is also an acceptable imaging modality, but CT provides more information about the amount of fluid and its location. Additionally, findings on CT will guide subsequent management. Endoscopic ultrasound (A) is primarily used in the setting of pancreatic or bile duct cancer to help determine resectability and look for adjacent enlarged lymph nodes. If the patient has evidence of infection, and a large fluid collection is found, a percutaneous drain should be placed. Bilious output suggests that bile has leaked (from the stump of the ligated cystic duct or worse from an injury to the common hepatic/bile duct). A HIDA scan (E) should be obtained next. If the common bile duct or common hepatic duct were inadvertently transected, the HIDA will show extravasation of tracer in the RUQ without tracer filling the small bowel. Such a finding would mandate exploratory laparotomy (B), and a loop of small bowel would need to be anastomosed to the proximal bile duct (hepaticojejunostomy). If on the other hand, the HIDA scan shows extravasation of tracer in the RUQ but tracer is seen in the small bowel, this confirms that the integrity of the main bile ducts. The most common cause for this latter finding is a cystic duct stump leak (as in the present case). Management is to perform ERCP with stenting (D) of the ampulla. This lowers the pressure in the biliary tree, creating a path of least resistance for the bile, thus permitting the cystic duct stump to seal.

\section{Answer C}

Patients with mild pancreatitis can often be managed being NPO along with intravenous hydration alone since recovery occurs rapidly, within 5-7 days, at which time oral intake can resume. However, patients with moderate-severe pancreatitis are unlikely to resume oral intake within 5-7 days, prompting the need for nutritional support. The most appropriate management is enteral nutrition. Enteral nutrition is provided through a nasojejunal tube, ideally placed past the ligament of Treitz as to not stimulate and irritate the pancreas. Enteral nutrition is preferred for those with a prolonged course of pancreatitis because it helps maintain the intestinal barrier and prevents bacterial translocation from the gut. In addition, enteral nutrition avoids the complications associated with parenteral nutrition including those secondary to venous access and blood stream infections. A 2010 meta-analysis of eight trials demonstrated that enteral nutrition significantly reduced mortality, multiorgan failure, infections, and the need for surgery as compared with those who received parenteral nutrition. Parental nutrition should only be initiated in patients who do not tolerate enteral feeding. In moderate-severe pancreatitis, oral feeding is not tolerated due to pain, nausea, or vomiting related to inflammation and edema causing gastric outlet obstruction, and should not be used. 


\section{Answer A}

Gallbladder polyps are usually incidental findings, most are asymptomatic, and the vast majority are benign. Risk of malignancy is related to polyp size. Polyps $<10 \mathrm{~mm}$ can be observed, whereas laparoscopic cholecystectomy is recommended for those $\geq 10 \mathrm{~mm}$. Open cholecystectomy (B) is not necessary for a polyp. Percutaneous gallbladder drainage (C) is indicated for the management of acalculous cholecystitis. Endoscopic ultrasound (D) would not provide any additional information. A follow-up ultrasound (E) would be appropriate for small polyps.

\section{Answer A}

The patient has severe pancreatitis as evidenced by having five Ranson's criteria. Mortality is markedly increased with three or more such criteria. Mortality in the first week is due to multisystem organ failure (pulmonary, renal, cardiac). ARDS occurs in a variety of settings, including severe pancreatitis. The classic presentation is severe hypoxia, with an associated respiratory alkalosis and a CXR that shows bilateral infiltrates that is often symmetrical. Therapy includes starting mechanical ventilation with positive end-expiratory pressure (PEEP). The overall mortality is very high $(>50 \%)$. Although a pulmonary embolism (B) may also present with hypoxia and a respiratory alkalosis, it is unlikely to present with bilateral infiltrates (the CXR is usually negative). Hospital-acquired pneumonia (C) would present with a productive cough, dyspnea, chills, pleuritic chest pain, decreased breath sounds, wheezing, and a CXR showing lobar consolidation. A patient with pulmonary edema (D) would have a CXR showing cephalization of vessels and Kerley B lines (i.e., prominent horizontal interstitial markings in lower lung fields), as well as an elevated CVP (>18 mmHg). Atelectasis (E) would appear as a white out of a lobe.

\section{Answer B}

Obstructive jaundice is an elevation of serum conjugated bilirubin due to inability to excrete it into the intestines via the biliary system. Thus there would be decreased available bilirubin in the intestine for intestinal bacteria to convert to urobilinogen and subsequently stercobilin. Although indirect and direct bilirubin are both elevated in obstructive jaundice, the direct component should be higher. Unconjugated bilirubin is bound to protein, and not filtered by the kidney. In patients with jaundice, conjugated bilirubin is elevated in the urine. The elevation in urine conjugated bilirubin gives it the brown ("tea-colored") discoloration. Elevation of transaminases out of proportion to alkaline phosphatase (E) would suggest hepatocellular injury.

\section{Answer B}

Smoking is a risk factor for pancreatic cancer. Pancreatic cancer is also more common in men, advanced age, in the obese, and in African Americans. Chronic pancreatitis is the strongest risk factor pancreatic cancer, although alcohol consumption (A) per se is not. A family history of prostate cancer (C) is not a risk factor for pancreatic cancer. Although malabsorption (D) and pancreatic enzyme supplementation (E) is frequently associated with patients that have chronic pancreatitis, they are not themselves directly linked to an increased risk for pancreatic cancer.

\section{Answer E}

UGI bleeding from varices most often are the result of alcohol-related liver cirrhosis with subsequent portal hypertension. This leads primarily to esophageal varices and less commonly to concomitant gastric varices. Isolated gastric varices are uncommon. They often arise in association with splenic vein thrombosis (SVT), which forces all the venous drainage of the spleen to travel through the short gastric veins resulting in large gastric varices that are at risk for rupture and bleeding. The most common cause of SVT is pancreatitis (acute and chronic). The most common cause of chronic pancreatitis is alcohol abuse. Peripancreatic inflammation can lead to occlusion of the splenic vein, which is posterior to the pancreas. SVT does not lead to esophageal varices because the collaterals do not involve the esophageal vasculature. Diagnosis of SVT can be made by duplex ultrasound of the splenic vein. It can also be detected on a venous phase CT scan. Splenectomy effectively eliminates the enlarged short gastric veins and thus cures the gastric varices. Gastric varices are particularly dangerous as they tend to cause massive bleeding. In addition, they do not respond well to standard treatment for esophageal varices such as banding (B) or sclerotherapy (C). Both liver transplantation (A) and TIPS (D) would reduce portal hypertension and thus help remedy esophageal varices but would be ineffective for isolated gastric varices in the setting of SVT.

\section{Answer $C$}

The first step in working up a pancreatic leak is to test the drained fluid for amylase. In addition, serum amylase level should be checked as well. If fluid amylase level is high and output levels are high $\left(>50 \mathrm{~cm}^{3} /\right.$ day), patient can be made NPO to decrease secretion of pancreatic fluid that accompanies oral intake. Imaging (A-B) can be done at a later time to evaluate adequacy of drainage. Octreotide (D) can be given to decrease pancreatic secretions, but it is not used routinely, and there is no evidence-based data that demonstrate costeffective efficacy of octreotide use in this setting. TPN (E) is not routinely needed in this setting.

\section{Answer D}

At this time CA 19-9 is not recommended for screening pancreatic cancer, and is also not a diagnostic test. However, 
many surgeons use CA 19-9 to monitor for recurrence following surgery.

\section{Answer B}

The patient presented has RUQ pain and tenderness, nausea, and an ultrasound demonstrating gallstones with a thickened gallbladder wall suggestive of acute cholecystitis. Mild elevations in ALT and AST can be expected with acute cholecystitis, as well as leukocytosis with a left shift. The patient should be admitted to the hospital, made NPO, given IV fluids, and IV antibiotics with gram negative, enterococcus, and anaerobic coverage. She should undergo laparoscopic cholecystectomy, ideally within $48 \mathrm{~h}(\mathrm{C}-\mathrm{E})$. Multiple studies have shown that delaying surgery is of no benefit and, in fact, makes the operation technically more challenging due to more scarring. Elective outpatient laparoscopic cholecystectomy is appropriate for patients with biliary colic (A).

\section{Answer A}

This patient has acute pancreatitis, most likely secondary to gallstones (most common cause). She has had prior episodes of pain after eating fatty food, which is characteristic of symptomatic gallstones. In addition, the lipase and amylase are elevated ( $3 \times$ greater than the upper limit of normal). Cholecystectomy is considered the standard of care in patients with gallstone pancreatitis because there is a high risk of recurrent pancreatitis. Although most centers have traditionally waited until all laboratory values have normalized for patients with mild disease (may take 5-7 days) (B), recent randomized studies have demonstrated that cholecystectomy can be safely performed within $48 \mathrm{~h}$ of admission (in patients with mild pancreatitis) regardless of whether laboratory values have normalized (D). Thus this patient should proceed with LC with IOC since she demonstrates no evidence of severe pancreatitis. On the other hand, with severe pancreatitis, such as necrotizing pancreatitis, delaying gallbladder removal until complete resolution of the pancreatitis is recommended. The gallstones that cause pancreatitis are usually small, and as such, in the majority of cases, the stone remains impacted very briefly, only transiently obstructing the ampulla of Vater, and soon after passes into the duodenum. As such persistent of a CBD stone is uncommon, and therefore ERCP is not usually needed $(\mathrm{C}, \mathrm{E})$.

\section{Answer A}

A diabetic patient presenting with symptoms suggestive of acute cholecystitis with high fevers, high WBC with left shift, and RUQ ultrasound demonstrating gas bubbles within the wall of the gallbladder should be suspected of having emphysematous cholecystitis, which is generally considered a surgical emergency. It is generally caused by gas-forming organisms, such as Clostridia and E. coli. Compared to acute cholecystitis, emphysematous cholecystitis is associated with a much higher mortality due to severe sepsis as the gallbladder becomes gangrenous. Broad-spectrum antibiotics (include high-dose penicillin or clindamycin to cover Clostridia) and fluid replacement are started immediately to stabilize the patient (B), but because of the risk of gangrene, these patients should undergo an immediate cholecystectomy. The diagnosis can be supported with CT scan of the abdomen (D) which will also demonstrate gas within the gallbladder wall. $\operatorname{ERCP}(\mathrm{E})$ is not indicated. Cholecystostomy (C) would not be an appropriate intervention for emphysematous cholecystitis as the necrotizing tissue infection would not be removed.

\section{Answer C}

A heavy calcified gallbladder is termed a porcelain gallbladder and is most commonly found incidentally on imaging for unrelated reasons. Patients are often asymptomatic. However, it is important to recognize that a porcelain gallbladder is associated with an increased risk of gallbladder adenocarcinoma. As such, the recommendation is that patients should undergo surgical management with laparoscopic cholecystectomy. Reassurance (A) or "watchful waiting" with annual CT scan (B) is not appropriate, even for asymptomatic patients, because of the risk for malignancy. There is no reason to suspect hyperparathyroidism, and so a serum calcium and PTH level would not be appropriate (D). ERCP with brushings is useful for suspected bile duct cancer, but not for suspected gallbladder cancer (E). 


\section{Lower Gastrointestinal}

\section{Areg Grigorian and Christian de Virgilio}

\section{Questions}

1. A 55-year-old male has been receiving serial ultrasound examinations to follow his abdominal aortic aneurysm (AAA). Over the past year, the aneurysm has rapidly enlarged to $5.8 \mathrm{~cm}$, and he undergoes endovascular abdominal aortic aneurysm repair (EVAR). The operation itself is uneventful. However, on postoperative day 1 , the patient develops a low-grade fever, left lower quadrant pain, and diarrhea that appears to be blood tinged. On physical examination, he has mild to moderate tenderness in the left lower quadrant without rebound or guarding. What is the next step in the workup?
(A) CT scan of the abdomen and pelvis with oral and IV contrast
(B) Exploratory laparotomy
(C) Formal mesenteric arteriography
(D) Flexible sigmoidoscopy
(E) Abdominal ultrasound

2. A 30-year-old man with colon cancer secondary to familial adenomatous polyposis (FAP) arrives for follow-up after receiving a total proctocolectomy with end ileostomy. He was found to have colon cancer after presenting at the age of 27 with unexplained rectal bleeding, diarrhea, and abdominal pain. Subsequent colonoscopy found multiple adenomatous polyps in his colon. He has a 5-year-old son, who is screened and is positive for the APC gene. What is the recommended screening for his son?
(A) Colonoscopy starting at age 20
(B) Flexible sigmoidoscopy starting at age 10
(C) Colonoscopy starting at age 50
(D) Annual fecal occult blood test
(E) Annual barium enema

3. A 66-year-old male presents with a large volume of maroon-colored stools combined with red blood. In the ED his blood pressure is $100 / 60 \mathrm{mmHg}$, and heart rate is $120 /$ min. Physical examination is unremarkable. Two large bore IVs are inserted, and 2 liters of normal saline are given, after which the patient's vital signs normalize. Laboratory tests are sent, including a type and cross. What is the next step in the management?
(A) Administer two units of $\mathrm{O}$ negative blood
(B) Place NG tube for aspiration
(C) Colonoscopy
(D) Exploratory laparotomy
(E) Place central line

4. A 27-year-old man arrives to the emergency department complaining of bloody diarrhea and rectal urgency. He reports a normal appetite, and has not lost any significant weight. After initial workup yields no findings, he is referred to a gastroenterologist for a colonoscopy. $\mathrm{He}$ is found to have pseudopolyps in his colon, and subsequent biopsy results confirm ulcerative colitis. $\mathrm{He}$ is started on corticosteroids and sulfasalazine, which is able to control his symptoms. Which of the following is true regarding colon cancer and screening in patients with ulcerative colitis?

(A) Screening for colon cancer is not necessary

(B) Screening colonoscopy with random biopsies 8 years after disease onset

(C) Screening colonoscopy with biopsy only if a suspicious polyp is seen

(D) Screening colonoscopy annually once diagnosis is established

(E) The risk of colon cancer is much less than with Crohn's disease

5. A 50-year-old man comes to the doctor complaining of painful defecation. On occasion, blood is found on the toilet paper after wiping. He has been experiencing this problem for months but felt too embarrassed to seek medical attention. He has a history of constipation and has tried multiple stool softeners but to no avail. Rectal examination shows enlarged anal papillae with an edematous and hypertrophic skin tag in the posterior anal midline, most suggestive of a chronic fissure. $\mathrm{He}$ is referred to a surgeon and scheduled to undergo a lateral internal sphincterotomy. What is the most common complication of this procedure?
(A) Bleeding
(B) Anorectal sepsis
(C) Fecal incontinence
(D) Fistula formation
(E) Nonhealing of fissure

6. An 88-year-old woman with multi-infarct dementia undergoes a coronary artery bypass with the left internal mammary artery used as a conduit for three-vessel disease. Her operation is a success, and she is transferred to the ICU to recover. She is receiving opioids for pain relief. Over the next few days, she develops a markedly distended abdomen with no bowel sounds, pain, or rigidity. She has also not had a bowel movement for the past $48 \mathrm{~h}$. Rectal examination does not demonstrate any retained stool. KUB demonstrates markedly distended colon with gas in the rectum, without air fluid levels. What part of the large bowel is the most likely to perforate?

(A) Cecum

(B) Transverse colon 

(C) Sigmoid colon
(D) Rectum
(E) There is very little risk of perforation

7. A 75-year-old man arrives to the ED with abdominal pain. He has never experienced this before but reports having left-sided abdominal pain over the last 2 days that is relieved temporarily after defecation. He has a temperature of $101.2{ }^{\circ} \mathrm{F}$ with a blood pressure of $142 / 88 \mathrm{mmHg}$ and pulse of $88 / \mathrm{min}$. His laboratory examination is significant for a WBC of $14 \times 10^{3} / \mu \mathrm{L}$ (normal $4.1-10.9 \times 10^{3} / \mu \mathrm{L}$ ) with $10 \%$ bands. CT imaging reveals focal sigmoidal wall thickening with significant paracolic inflammation. He was treated as an in-patient for 3 days and discharged home without complications. Which of the following is the most appropriate follow-up option?
(A) High-fiber diet alone
(B) CT scan 2 weeks after resolution
(C) Elective sigmoid colectomy
(D) Barium enema
(E) Colonoscopy

8. A 28-year-old woman presents to the ED because of abdominal pain that started $2 \mathrm{~h}$ ago. She has nausea and vomited twice in the last hour. She reports that the pain is predominantly in the right lower quadrant. On physical exam, her blood pressure is $120 / 60 \mathrm{mmHg}$, heart rate is $100 / \mathrm{min}$, and she is afebrile. She is very tender in the RLQ on palpation at McBurney's point and has pain on passive extension of the right hip. Her WBC is $14 \times 10^{3 /}$ $\mu \mathrm{L}$ (normal $4.1-10.9 \times 10^{3} / \mu \mathrm{L}$ ), hemoglobin is $12 \mathrm{~g} / \mathrm{dL}$ $(12-15.2 \mathrm{~g} / \mathrm{dL})$, hematocrit is $36 \%(37-46 \%)$, and platelet count is $250,000(140-450,000)$. Her urinalysis shows $1+$ white blood cells. Which of the following is the best next step in management?
(A) Admit for observation
(B) CT scan of the abdomen
(C) Laparoscopic appendectomy
(D) B-hCG
(E) Broad-spectrum antibiotics

9. A 38-year-old presents with symptoms and signs of acute appendicitis and undergoes laparoscopic appendectomy. At surgery the terminal ileum and cecum appear to be red and inflamed. The appendix is removed uneventfully. Final pathology of the appendix demonstrates no evidence of acute appendicitis. Two weeks later, he presents back to the ED with feces draining from his right lower quadrant wound. Which of the following is the most likely explanation for why the drainage may not spontaneously stop?

(A) A distal colonic obstruction

(B) Chronic inflammation
(C) An occult intra-abdominal abscess

(D) A retained sponge in the abdomen

(E) A missed malignancy

10. Which of the following is most likely to require urgent colectomy?
(A) Cecal volvulus
(B) Sigmoid volvulus
(C) Acute diverticulitis
(D) Cecal adenocarcinoma
(E) Pseudomembranous colitis

11. A 55-year-old man starts a new security job that requires a physical exam by a doctor. He has no past medical history, and everyone in his family is healthy. He has a well-balanced diet and exercises every day. He is evaluated during a routine examination which includes a discussion of health maintenance issues. He is surprised to hear that it is recommended for a man of his age to get a colonoscopy. He asks if there are any other options available. Which of the following is an appropriate recommendation with respect to this health maintenance screening strategy?

(A) Fecal occult blood test (FOBT) every 5 years

(B) Annual rectal examination

(C) Flexible sigmoidoscopy every 5 years +FOBT every 3 years

(D) Annual CT colonography

(E) Annual CEA levels

12. A 90-year-old man with Alzheimer's disease arrives from a nursing home with abdominal distention and pain for the past $12 \mathrm{~h}$. He takes hydrochlorothiazide, donepezil, aspirin, and docusate. His physical exam reveals a temperature of $102^{\circ} \mathrm{F}$, blood pressure $90 / 70 \mathrm{mmHg}$, and pulse $112 / \mathrm{min}$. His abdomen is rigid and diffusely tender with rebound and guarding. Laboratory examination is significant for $\mathrm{WBC}$ of $15 \times 10^{3} / \mu \mathrm{L}$ (normal $4.1-$ $\left.10.9 \times 10^{3} / \mu \mathrm{L}\right)$, BUN of $25 \mathrm{mg} / \mathrm{dL}(7-21 \mathrm{mg} / \mathrm{dL})$, and creatinine of $1.8 \mathrm{mg} / \mathrm{dL}(0.5-1.4 \mathrm{mg} / \mathrm{dL})$. X-ray shows a markedly distended colon with a bent-inner tube sign. Following IV fluids and antibiotics, what is the best next step in management?

(A) Decompression with colonoscopy

(B) CT scan of the abdomen and pelvis

(C) Exploratory laparotomy

(D) Placement of a rectal tube

(E) Admit to hospital for close observation

13. A 44-year-old man presents with right lower quadrant pain without rebound, nausea, and vomiting. CT scan shows bowel wall thickening near the ileocecal valve. He is scheduled for an appendectomy. Final pathology confirms acute appendicitis. In addition, an incidental $2.5 \mathrm{~cm}$ 
carcinoid tumor is found at the tip of the appendix. What is the most appropriate next step in management?
(A) Observation
(B) Colonoscopy within the next 6 months
(C) UGI with small bowel follow through
(D) Chest X-ray
(E) Right hemicolectomy

14. A 64-year-old male returns for follow-up six months after successful sigmoid colectomy for colon cancer. Which of the following can lead to a falsely elevated serum CEA level?
(A) Chronic wound infection
(B) Smoking
(C) Hyperglycemia
(D) Age
(E) Postoperative chemotherapy

15. A 4-year-old boy presents to the emergency department with right lower quadrant pain, nausea, and anorexia. On physical examination, his bowel sounds are absent, and he has marked tenderness in the right lower quadrant with guarding. The remainder of the abdominal exam is negative. Laboratory values reveal a leukocytosis with a left shift. What is the most likely initiating event for his acute condition?
(A) Lymphoid hyperplasia
(B) Fecalith
(C) Parasitic infection
(D) Enlarged mesenteric lymph nodes
(E) Foreign body ingestion

16. A 50-year-old woman undergoes screening colonoscopy. During the procedure, she is found to have a dark discoloration of the entire colon with lymph follicles shining through as pale patches. What is the most likely underlying etiology?
(A) Drinking lots of prune juice
(B) Colon cancer
(C) Laxative abuse
(D) Normal anatomic variation
(E) Inflammatory bowel disease

17. A 88-year-old man with Parkinson's disease and COPD is brought to the ED from a skilled nursing facility because his nurse noticed bright red blood in his adult diapers. His medications include hydrochlorothiazide, metformin, levodopa, salmeterol, and docusate. On admission, he is afebrile with normal blood pressure and pulse. Nasogastric tube (NGT) aspiration returns yellow bile. Colonoscopy shows bright red blood within the colon, multiple diverticula, but due to the large amount of stool and blood clots, no active bleeding sources are able to be seen. He continues to have blood per rectum over the next hour. Blood pressure is $120 / 70 \mathrm{mmHg}$ and heart rate is $90 / \mathrm{min}$. Which of the following is the most appropriate next step in management?
(A) Exploratory laparotomy
(B) Transfuse two units of packed red blood cells
(C) 99mTc red blood cell (RBC) scintigraphy
(D) Esophagogastroduodenoscopy (EGD)
(E) Omeprazole and antibiotics

18. A 62-year-old female arrives to the ER with acute abdominal pain. She has a past medical history significant for diverticulitis. She is diagnosed with uncomplicated diverticulitis and managed with bowel rest and antibiotics. A year later, she has another episode of diverticulitis that was again successfully managed on an outpatient basis. Which of the following is this patient at higher risk for developing?
(A) Crohn's disease
(B) Ulcerative colitis
(C) Colon cancer
(D) Anal cancer
(E) Stricture formation

19. Endocarditis secondary to which of the following organisms is associated with colon cancer?
(A) Streptococcus bovis
(B) Clostridia septicum
(C) Steptococcus bovis and Clostridia septicum
(D) Diphyllobothrium latum
(E) Cryptococcus neoformans 


\section{Answers}

\section{Answer D}

Ischemic colitis is one of the complications that can occur after AAA repair. The etiology is thought to be due to the fact that the inferior mesenteric artery (IMA) is ligated (with open repair) or occluded (with endovascular repair). In the majority of patients, there is no consequence from ligating the IMA. Depending on collateral blood supply, the left colon may develop mucosal or full-thickness ischemia. Patients present with varying degrees of left lower quadrant pain, fever, diarrhea, or frankly bloody stool. Ischemic colitis is confirmed by flexible sigmoidoscopy that will demonstrate inflamed, friable mucosa, or full-thickness necrosis (bear in mind that a scope within the lumen cannot necessarily determine full-thickness necrosis). Treatment of ischemic colitis begins with placing the patient NPO and administering IV fluids and broad-spectrum antibiotics. If there is evidence of sepsis and/or peritonitis, the patient will require exploratory laparotomy (B), colonic resection, and a proximal colostomy. Since the cause of the ischemic colitis (ligation of the IMA) is known, and cannot be reversed, CT (A), formal mesenteric arteriography $(\mathrm{C})$, and ultrasound $(\mathrm{E})$ are not helpful.

\section{Answer B}

Familial adenomatous polyposis (FAP) is an autosomal dominant condition in which patients develop hundreds to thousands of polyps in the colon, which if left untreated, has a $100 \%$ chance of developing into cancer by age 50 . The mutation is in the adenomatous polyposis coli (APC) gene on chromosome 5 . A child who has a parent with the mutation has a $50 \%$ chance of inheriting the syndrome. Thus APC gene testing is recommended. If the child tests positive, screening with flexible sigmoidoscopy should begin at age 10 (as polyps can develop even in the teen years). Once a polyp is seen, surgery is recommended to remove the colon. Since the polyps begin on the left side of the colon, a full colonoscopy $(\mathrm{A}, \mathrm{C})$ is not required for screening. Annual fecal occult blood test (D) or barium enema (E) are not adequate screening tools in FAP.

\section{Answer B}

Prior to initiating a workup for GI bleeding, it is important to determine if the source is the upper or lower GI tract. If the patient is vomiting blood or has coffee ground emesis, then the source is clearly an UGI one. Since a massive UGI bleed can cause bleeding per rectum, it is imperative that an UGI source is ruled out first. This is achieved by placing an NG tube and aspirating for blood or coffee grounds. If it returns blood, an upper endoscopy should be performed first. If the aspirate returns bile, then an UGI bleed is ruled out. Since the patient responded to a fluid challenge, a blood transfusion (A) is not necessary. Colonoscopy (C) is not yet indicated nor is surgery (D). A central line (E) should be considered if ongoing massive fluids/blood are needed.

\section{Answer B}

Patients with IBD are at increased risk of colon cancer. The risk is much greater for ulcerative colitis (UC) than with Crohn's and is related to the duration of illness and the extent of disease. For UC, the risk is low in the first 10 years of the disease $(2-3 \%)$ but grows to $1-2 \%$ per year afterwards. UC patients should begin getting screened 8 years after disease onset and continue getting screened every 1-2 years after. Random biopsies are necessary in patients with UC undergoing screening with colonoscopy because in these patients, cancers do not follow the typical progression from polyp to cancer. A proctocolectomy removes the entire rectum and colon, which prevents patients with UC from developing cancer and no further surveillance is required.

\section{Answer C}

Anal fissure is the most common cause of minor painful rectal bleeding (hemorrhoids usually cause minor painless bleeding). To get an anal fissure, one needs two things: anal trauma (from hard stools) and a hypertonic/hypertrophied internal sphincter. The hard stool tears the anoderm, most often in the posterior midline (most vulnerable to damage due to a relatively diminished blood supply). Patients typically present with painful defecation and blood found on tissue after wiping. Chronic fissures become deeper and will have enlarged anal papillae with hypertrophic and edematous skin tags (sentinel pile). Medical management includes sitz baths (relaxes sphincter), fiber, and stool softeners. If this treatment fails, nitroglycerine ointment (limited by side effect of headaches) and botulinum toxin (injected into the internal sphincter) are the next steps in management. Patients that fail medical therapy can be considered for surgical treatment with lateral internal sphincterotomy, which is able to cure $95 \%$ of cases. The risk of incontinence ranges from $5 \%$ to $15 \%$. The other complications listed are uncommon (A-B, D-E).

\section{Answer A}

Ogilvie's syndrome is a pseudo-obstruction of the colon that is associated with bedridden, neurologically impaired or older patients, opiate use, recent surgery, trauma, or infection. The mechanism for this colon dysfunction is unknown. X-ray films will predominantly show a markedly colon without evidence of a bowel obstruction. Management consists of removing any drugs that may interfere with gut motility (e.g., opiates) and replacement of electrolytes (especially potassium). A colon larger than $10 \mathrm{~cm}$ is at risk for perforation and requires decompression with colonoscopy and neostigmine. Due to the law of Laplace (tension=pressure $\mathrm{x}$ radius), the cecum, being the part of the colon with the largest diameter, is the most common site for perforation (B-E). 


\section{Answer E}

Diverticula occur as a result of herniation of mucosa through the colonic wall at sites where arteries enter the muscular layer. The incidence of diverticula increases with age, and it is believed to be present in $35 \%$ of the population. When diverticula get infected (diverticulitis), often on the left side, patients can present with fever, leukocytosis, and LLQ pain that may be temporarily relieved following defecation. This patient has a moderate case of diverticulitis given the CT findings of significant inflammation in the sigmoid colon, fever, and leukocytosis. Treatment initially consists of bowel rest and IV antibiotics. Patients with acute diverticulitis should receive a follow-up colonoscopy to rule out malignancy (as the CT cannot readily distinguish diverticulitis from colon cancer). Colonoscopy should not be performed during the same hospitalization as the insufflation of air may lead to free perforation. Barium enema (with or without sigmoidoscopy) (D) is not a recommended screening tool for colon cancer. In addition to a colonoscopy, a fiber-rich diet (A) should be encouraged for all patients with diverticulitis to reduce the incidence of diverticula. Follow-up CT scan (B) is unlikely to provide any additional information other than confirming diverticula. Surgery $(\mathrm{C})$ is recommended for cases with significant complications (e.g., complete obstruction, free perforation with diffuse peritonitis) and those that have failed medical management.

\section{Answer D}

The differential diagnosis of RLQ pain in women is more extensive than for men and should include ruptured ectopic pregnancy, ruptured cyst, ovarian torsion, and pelvic inflammatory disease. A B-hCG test should always be ordered in a woman of child-bearing age with abdominal pain to rule out pregnancy. If positive, a ruptured ectopic pregnancy should be high on the differential. Although the patient presented has McBurney's point tenderness and an elevated WBC, laparoscopic appendectomy $(\mathrm{C})$ would not be recommended as of yet until the B-hCG is obtained. In addition, in women, ultrasonography is highly useful to rule out the aforementioned differential. CT scan (B) is not necessary to confirm the diagnosis of acute appendicitis. Rather it is used if the diagnosis is in question. Admission for observation (A) would not be appropriate. Do not assume that pyuria rules out appendicitis (an inflamed appendix can cause sterile pyuria).

\section{Answer B}

A rare complication after appendectomy is a cecal fistula. The findings on laparoscopy (inflamed terminal ileum and cecum) combined with a normal appendix indicate that the patient's actual diagnosis is likely Crohn's disease which can mimic appendicitis (in this setting, it is termed regional enteritis). If regional enteritis is found instead of appendicitis at the time of laparoscopy, the appendix is removed even if it looks normal, provided the cecum is not involved in the inflammation. This prevents confusion in case the patient presents again with RLQ pain in the future. However, if the cecum is also inflamed in the setting of regional enteritis, the appendix should not be removed, as there is a risk that the stump of the appendix will blow out and form a fistula (as in the present case). Conditions that prevent fistulas from spontaneously closing can be remembered by the acronym "HIS FRIEND": high fistula output ( $>500 \mathrm{~cm}^{3} /$ day), inflammatory bowel disease, short fistula $(<2.5 \mathrm{~cm})$, foreign body, radiation, infection, epithelialization, neoplasm, and distal obstruction. Although all the choices above can all contribute to non-closure of a fistula, chronic inflammation (from inflammatory bowel disease) is the most likely one.

\section{Answer A}

Cecal volvulus is due to a failure of fixation of the right colon (due to a congenital malrotation). Once diagnosed, treatment is to perform an urgent right colectomy, as it does not respond well to nonoperative management (it cannot effectively be detorsed with colonoscopy). Provided there is no evidence of ischemic bowel, sigmoid volvulus (B) is most often managed with initial endoscopic decompression, followed by a semielective sigmoid colectomy after performing a bowel prep. Most patients with diverticulitis (C) can be managed via a combination of antibiotics, bowel rest, and subsequent dietary modification (high fiber). Due to the large diameter of the cecum and the liquid nature of stool at that location, cecal adenocarcinoma (D) rarely presents with a large bowel obstruction (which would be the main indication for urgent surgery with colon cancer). Rather, it presents most commonly with iron deficiency anemia. Pseudomembranous colitis (E), due to Clostridia difficile, is successfully managed in most patients with oral metronidazole (and stopping the offending antibiotics). Other antibiotic choices include vancomycin and fidaxomicin. In rare cases, the colitis can progress to life-threatening sepsis (termed toxic colitis) that requires emergency total colectomy.

\section{Answer C}

A colonoscopy is considered the gold standard because it can visualize the entire colon, identify small polyps/adenomas, and take biopsies. However, not all patients are comfortable with this test and could benefit from learning about other options. Flexible sigmoidoscopy every 5 years with FOBT every 3 years is an alternative screening modality recommended by the American College of Radiology Joint Task Force. An annual FOBT (not every 5 years) is another appropriate alternative (A), but abnormal results should always be followed up with a colonoscopy. Annual rectal examination (B) is not considered adequate screening because of poor sensitivity $(9 \%)$. CT colonography done every 5 years 
(not annually) (D) is as likely as colonoscopy to detect lesions $10 \mathrm{~mm}$ or larger but may be less sensitive for smaller adenomas. Serum CEA level (E) is used as a marker for colon cancer recurrence following surgery; however, CEA level lacks both sensitivity and specificity (it is elevated with other cancers and with benign conditions), such that it should never be used for colon cancer screening.

\section{Answer $C$}

Sigmoid volvulus occurs in debilitated and/or psychiatric patients. The twist in the colon leads to a closed loop obstruction which, if left untreated, leads to colonic ischemia, sepsis, and eventual colonic gangrene and perforation. Patients present with abdominal distention, pain, constipation, and vomiting. X-ray films may show an air filled closed loop of massively distended colon, referred to as a "bent-inner tube" or "coffee-bean" sign. Most patients can be managed by decompression with colonoscopy. However, this patient demonstrates evidence that the colon is already ischemic/ gangrenous (fever, tachycardia, hypotension, peritonitis, leukocytosis). Thus colonic decompression (A) is contraindicated. Following fluid resuscitation and antibiotics, the patient requires urgent exploratory laparotomy with likely sigmoid colon resection and proximal colostomy. Similarly, with peritonitis and the classic plain X-ray finding, CT scan is unnecessary (B) and in fact may cause renal injury given his pre-renal azotemia. Placing a rectal tube (D) and admitting to the hospital (E) without surgery would not be appropriate.

\section{Answer $E$}

It is imperative that following appendectomy, the appendix is inspected and histologically examined by a pathologist as an appendiceal tumor is sometimes discovered incidentally. The most common appendiceal tumor is mucinous adenocarcinoma (it was previously thought to be carcinoid) with the majority of these tumors located at the tip of the appendix. If the tumor is $\leq 1 \mathrm{~cm}$, an appendectomy is considered appropriate definitive management. For tumors larger than $1 \mathrm{~cm}$ and located at the base of the appendix or tumors $\geq 2 \mathrm{~cm}$ and located at the tip of the appendix, a right hemicolectomy is indicated. The other choices would not be appropriate (A-D).

\section{Answer B}

Carcinoembryonic antigen (CEA) is a glycoprotein, which is present in normal mucosal cells, but increased amounts are associated with adenocarcinoma, especially colorectal cancer. The major role of CEA in colon cancer patients is to allow clinicians to monitor for recurrence after intended curative treatment. Typically, patients achieve normal levels about 4-6 weeks after surgical intervention. Smoking up to $4 \mathrm{~h}$ prior to checking serum CEA can falsely elevate levels. CEA levels are also elevated with other cancers (e.g., medullary carcinoma of the thyroid, pancreatic, gastric cancer) as well as benign conditions (IBD, pancreatitis, cirrhosis, and smoking), but not with the other choices provided $(\mathrm{A}, \mathrm{C}, \mathrm{D}, \mathrm{E})$. It is recommended to counsel all patients to quit smoking as it can affect postoperative monitoring and improves overall health.

\section{Answer A}

Acute appendicitis is triggered by an obstruction of the appendiceal lumen. In adults the obstruction is most commonly caused by a fecalith (B). However, in children the obstruction is most often due to lymphoid hyperplasia within the appendix that may be triggered by an antecedent viral infection. Thus patients may have a history of a recent viral infection prior to the acute appendicitis. Parasites (C), such as pinworm, and foreign body ingestion (E) can rarely obstruct the appendiceal lumen and cause appendicitis. Acute mesenteric adenitis (D) can mimic acute appendicitis. However, this is less common, and the vast majority present with mild diffuse abdominal pain as opposed to localized pain and tenderness at McBurney's point.

\section{Answer $C$}

This patient most likely has melanosis coli, also known as pseudomelanosis coli, secondary to laxative abuse. This benign condition is often discovered incidentally during colonoscopy and is considered a disorder of pigmentation of the colonic wall. The dark discoloration is a result of lipofuscin in macrophages, and not melanin. Patients can be asymptomatic or sometimes present with watery or nocturnal diarrhea. The other options (A, B, D) are not consistent with the colonoscopic findings. Inflammatory bowel disease (IBD) (E) may show cobblestone mucosa, strictures, and/or pseudopolyps and a colon devoid of haustra.

\section{Answer C}

This patient is suffering from a lower GI bleed (LGIB). The risk of LGIB increases with age, a trend that is driven by the age-associated increase in the incidence of diverticular hemorrhage, which is the most common cause of LGIB. Colonoscopy is indicated, but in the acute bleed setting may not demonstrate the source of bleeding. Since the bleeding has not stopped and has not been localized, additional studies are indicated. Options include radiolabeled RBC scintigraphy or angiography. Scintigraphy has the advantage of being noninvasive, is more sensitive (it detects less brisk bleeding), and can be easily repeated. It is utilized when the patient is actively bleeding (provided they are stable) when colonoscopy cannot identify the source. Exploratory laparotomy $(\mathrm{A})$ would be indicated if the patient is hemodynamically unstable from a massive LGIB as there is no time to identify the exact source. Transfusion (B) is not indicated give that the patient responded to IV fluids. EGD would be the first-line study for an upper GI bleed (D). Omeprazole 
and antibiotics (E) would be indicated in a patient suspected of having ulcers secondary to $H$. pylori.

\section{Answer $\mathrm{E}$}

One of the complications that may develop from recurrent bouts of diverticulitis includes stricture formation. In response to repeated inflammatory insults to the colon, a portion may become scarred and subsequently narrowed. These patients can present with a decreased caliber of stool, ileus, or bowel obstruction. Barium enema or colonoscopy is often used to diagnose patients. Diverticulitis does not increase the risk of developing IBD, colon cancer, or anal cancer (A-D).

\section{Answer C}

Streptococcus bovis and Clostridia septicum are both associated with colon cancer. The clinical context $S$. bovis comes up in most often is in that of patients suffering from endocarditis. C. septicum can spread hematogenously to muscle tissue and is also associated with the concomitant presence of hematologic malignancy. All patients found to be infected with these organisms should be scheduled for a colonoscopy to rule out colorectal malignancy. Diphyllobothrium latum (fish tapeworm) (D) and Cryptococcus neoformans (E) have not been linked to an increased risk of developing colon cancer. 


\section{Neurosurgery}

\section{Areg Grigorian and Christian de Virgilio}

\section{Questions}

1. A 32-year-old man presents with progressive frontal headaches. His symptoms started 2 months ago and often wake him up from his sleep. His vital signs are stable, and neurologic examination reveals no focal deficits. MRI brain imaging reveals a mass lesion, and subsequent biopsy is consistent with a type IV astrocytoma. Which of the following is true regarding this patient's illness?

(A) It is considered the most common primary malignant brain tumor

(B) Prognosis is good since the tumor is slow growing

(C) The biopsy should demonstrate psammoma bodies

(D) This tumor does not cross the corpus callosum

(E) Interferon beta and glatiramer acetate are used in the management of this tumor

2. Which of the following is true regarding diffuse axonal injury?

(A) It is often associated with a lucid interval

(B) Blurring of gray-white junctions is commonly found on imaging

(C) It occurs following a tensile force

(D) Persistent vegetative state is rare

(E) Patients often have hyperdense fluid in the ventricles, sulci, and cisterns

3. A 55-year-old female restrained driver arrives to the ED following a MVC. She sustained hyperextension of the cervical spine and hit her chest on the steering wheel. Neurologic examination shows spastic paraplegia and loss of pain sensation in both upper extremities. She is able to move both her legs and can differentiate vibratory sensation and light touch in both feet. What is the most likely etiology for her acute condition?
(A) Central cord syndrome
(B) Anterior spinal artery syndrome
(C) Brown-Sequard syndrome
(D) Cauda equina syndrome
(E) Subacute combined degeneration of the spinal cord

4. Which of the following findings would be expected in a patient presenting with a transtentorial (uncal) herniation?
(A) Loss of gag reflex
(B) Diplopia on attempted lateral gaze
(C) Medial rectus palsy on attempted lateral gaze
(D) Ptosis and a "down and out" eye
(E) Paralysis of the sternocleidomastoid muscle

5. Which of the following is the most common brain cancer?
(A) Lung metastasis
(B) Glioblastoma multiforme
(C) Colon metastasis
(D) Breast metastasis
(E) Astrocytoma

6. A 64-year-old homeless man is brought to the ED by paramedics after being found confused on the street. He has bruises on his head and arms with a few that appear to be new. He has alcohol on his breath. He is awake in the ED but unable to follow commands or answer questions. CT scan of the head without contrast demonstrates a crescent-shaped lesion. Which of the following is most associated with this patient's condition?
(A) Family member with polycystic kidney disease
(B) Congenital malformation
(C) Torn bridging veins
(D) Injured middle meningeal artery
(E) Temporal bone fracture

7. In a patient with an isolated head injury and concerns for increased intracranial pressure, which of the following would have the most potential to benefit the patient?
(A) Hypertonic (3\%) saline solution
(B) Ventilation with permissive hypercapnia
(C) Trendelenburg position of bed
(D) Intermittent D50 (dextrose) boluses
(E) Nitroprusside drip

8. A 20-year-old female arrives to the ED after slipping and hitting her head on ice and briefly losing consciousness. In the ED, she vomits twice. She denies amnesia. Her temperature is $98.9^{\circ} \mathrm{F}$, blood pressure is $110 / 80 \mathrm{mmHg}$, and pulse is $80 / \mathrm{min}$. Her GCS is 15 . Her physical exam is normal with no papilledema. What is the most appropriate next step in management?

(A) Admit for observation and order CT scan of the head only if she develops a neurologic deficit

(B) Admit for observation and start corticosteroids

(C) Order CT scan of the head without contrast now

(D) Discharge home now

(E) Discharge home with a tapered dose of corticosteroids

9. A 48-year-old woman arrives for her 6-month follow-up after undergoing breast-conserving therapy to treat stage IIA breast cancer. Her only postoperative complication has been red patches of skin on her chest following radiation therapy. She does not complain of any pain or pruritus but does report having intense headaches in the mornings when she wakes up. Her temperature is $99.1^{\circ} \mathrm{F}$, 
blood pressure is $110 / 80 \mathrm{mmHg}$, and pulse is $90 / \mathrm{min}$. Her physical examination is normal. She is worried that the red patches on her chest are suggestive of something more serious. Which of the following studies would be the most appropriate next step in management?
(A) PET
(B) Cerebral angiogram
(C) Skin biopsy
(D) Carotid duplex
(E) CT scan of the head

10. A 29-year-old woman arrives to the ED following a jet ski accident. She has multiple bruises on her head, torso, and legs. She did not lose consciousness. Her temperature is $99.8^{\circ} \mathrm{F}$, blood pressure is $108 / 78 \mathrm{mmHg}$, and pulse is $102 / \mathrm{min}$. She only opens her eyes when you speak loudly to her. She is confused and screams in pain and withdraws when you palpate her left lower leg. Her imaging is pending. What is her calculated Glasgow Coma Scale?
(A) 6
(B) 8
(C) 10
(D) 12
(E) 14

11. A 22-year-old patient arrives to the ED after falling off his bicycle and hitting his head. In the ED, he is clearly intoxicated and his speech is slurred. He denies any neck pain but is not cooperative during the exam. He is placed in a cervical spine collar. What is the optimal method for clearance of the cervical spine in this patient?
(A) CT scan of the neck only
(B) MRI of the neck only
(C) MRI of the neck followed by a CT only if the MRI is negative
(D) CT scan of the neck followed by MRI only if the $\mathrm{CT}$ is negative
(E) The C-spine can be cleared using physical examination

12. A 50-year-old woman arrives to the ED after she was witnessed having two generalized tonic-clonic seizures over 15 min without recovering consciousness between episodes. Her seizures are resolved by the time she gets to the hospital. She had a toe removed for a skin tumor 10 years ago but does not remember any details. Review of systems reveals a loss of nearly $15 \mathrm{lb}$ over the last 2 months. She has no other complaints. CT scan of the head reveals a solitary mass in the gray-white junction of the left temporal lobe with some surrounding edema. After further imaging and work-up is done, no other lesions are identified. Which of the following most likely represents the mass?
(A) Type IV astrocytoma
(B) Metastasis
(C) Oligodendroglioma
(D) Meningioma
(E) Ependymoma

13. A 29-year-old male with no past medical history presents to the ED with the worst headache of his life. He was watching television when the headache started and is unlike any headache he has experienced before. It is located near the back of his head. He reports that his father had a kidney transplant at a young age. He is managed appropriately for his acute condition. His recovery is complicated by progressive lethargy, agitation, and eventual coma. Which of the following would most likely explain this complication?
(A) Nephrogenic diabetes insipidus
(B) Central (neurogenic) diabetes insipidus
(C) Poor oral intake
(D) Cerebral salt-wasting syndrome
(E) Thyroid dysfunction

14. A 31-year-old male with HIV presents with headaches and night sweats. He is poorly compliant with his medications. His headaches are severe in the morning but subside throughout the day. He has no other complaints. His last CD-4 count was $95 / \mathrm{mm}^{3}$. His temperature is $98.9^{\circ} \mathrm{F}$, blood pressure is $110 / 76 \mathrm{mmHg}$, and pulse is $88 / \mathrm{min}$. His physical exam is normal. CT scan of the head shows a ringenhancing lesion near his right primary motor cortex. What is the best next step in management?
(A) Radiation
(B) Chemotherapy and radiation
(C) Pyrimethamine and sulfadiazine
(D) Stereotactic brain biopsy
(E) Restart highly active antiretroviral therapy (HAART)

15. A 61-year-old man with a past medical history of opioid dependence and diabetes presents with focal back pain and right leg weakness. He does not recall any recent trauma. He reports having "back surgery" nearly 20 years ago but does not remember why. He also reports helping his friend move furniture last week. His temperature is $101.4^{\circ} \mathrm{F}$, blood pressure is $104 / 76 \mathrm{mmHg}$, and pulse is $102 / \mathrm{min}$. Physical examination is significant for focal pain on palpation of his lower lumbar spine. Laboratory examination is significant for elevated ESR. Imaging is obtained. What is the best management approach for this condition?

(A) Nonsteroidal anti-inflammatory drugs (NSAID)

(B) High-dose intravenous corticosteroids

(C) Long-term antibiotics alone 
(D) Physical therapy and education

(E) Long-term antibiotics with surgical drainage

16. A 40-year-old female presents to the doctor with progressive weakness in both her arms for the past year. She is healthy and does not take any medications but reports being hospitalized 5 years ago for 2 days following a MVC in which she sustained severe and sudden whiplash injury. On physical examination, she has atrophy of the intrinsic muscles of both hands and an inability to differentiate between hot and cold in bilateral upper extremities. All other sensations are intact. What is the most likely underlying etiology?
(A) Spinal spondylosis
(B) Brachial plexus injury
(C) Syringomyelia
(D) Autoimmune condition
(E) Herniated disk 


\section{Answers}

\section{Answer $A$}

Glioblastoma multiforme is a type IV astrocytoma and considered to be the most common primary malignant brain tumor in the United States. This tumor has a grave prognosis with life expectancy estimated to be $<1$ year from the time of diagnosis (B). On gross appearance, it can cross the corpus callosum (butterfly glioma) (D). On histologic appearance, it will stain with GFAP. Psammoma bodies are spindle cells concentrically arranged in a whorled pattern and are characteristic of a meningioma (C). Interferon beta and glatiramer acetate have been shown to decrease the frequency of future attacks in patients with multiple sclerosis (E).

\section{Answer B}

Patients with diffuse axonal injury following rapid deceleration trauma typically suffer instantaneous loss of consciousness followed by a persistent vegetative state (D). Prognosis is very poor. It occurs as a result of shearing forces $(C)$. A CT scan of the head will show numerous minute punctate hemorrhages with blurring of gray-white junctions. A lucid interval (A) is often associated with an epidural hematoma. Hyperdense fluid in the ventricles, sulci, and cisterns (E) would be suggestive of a subarachnoid hemorrhage.

\section{Answer A}

This patient has central cord syndrome which classically occurs following hyperextension of the cervical spine but may also result from hyperflexion. The lateral corticospinal fasciculus is home to the motor tracts of the lower extremities and is often spared in central cord syndrome. The medial aspect hosts the motor tracts for the upper extremities and is disproportionately affected, often with profound hand weakness. Most patients regain functional recovery in a predictable fashion starting first with the lower extremities (if affected), then bladder/bowel function, and finally the upper extremities. Anterior spinal artery syndrome (B) results from a compressed anterior spinal artery. This presents with bilateral loss of motor, pain, and temperature below the lesion with partial sensory sparing (e.g., proprioception, vibration) since the posterior dorsal columns remain viable. BrownSequard syndrome presents with ipsilateral motor weakness with associated upper motor neuron signs (spasticity, hyperreflexia, clonus, and positive Babinski sign) and touch/ proprioception loss below the level of the injury, also contralateral loss of pain and temperature sensation beginning one or two dermatome levels below the level of the injury. Cauda equina syndrome (D) may occur in a traumatic setting following compression of the lumbar nerve roots by a spinal fracture. Patients will present with perineal sensory deficit (saddle anesthesia), bowel/bladder incontinence, and pain/ weakness in the lower extremities. Subacute combined degeneration of the spinal cord (E) is the term used to describe the neurologic deficits (e.g., peripheral sensory deficits, loss of deep tendon reflexes) seen in patients with B12 deficiency.

\section{Answer D}

The uncus is a part of the medial temporal lobe and can herniate through the tentorium and compress the midbrain and brainstem during significant brain swelling. This will present with features that can be explained by local compression of nearby structures by the herniated uncus (e.g., mydriasis or "blown pupil," ipsilateral ptosis, and "down and out" eye). Loss of gag reflex (A) would be expected in patients with an injured glossopharyngeal nerve (CN IX). This can occur in a patient with a posterior fossa tumor. Diplopia on attempted lateral gaze $(B)$ is expected in patients with injury to the abducens nerve (CN VI), as in those patients with cavernous sinus malignancy. Medial rectus palsy on attempted lateral gaze (C) is commonly seen in patients with multiple sclerosis that have medial longitudinal fasciculus (MLF) syndrome. Paralysis of the sternocleidomastoid muscle would be expected in patients with a spinal accessory nerve injury.

\section{Answer A}

Malignant brain tumors are more commonly metastatic than primary (B, E). An estimated 24-45\% of all patients with cancer have metastatic lesions to the CNS. Nearly half of all brain metastasis are attributed to lung cancer. The second most common cancer to metastasize to the brain is breast (15\%) (D), followed by genitourinary (11\%), melanoma (9\%), and head and neck cancers (6\%). Prostate and colon cancers $(\mathrm{C})$ do not commonly metastasize to the brain.

\section{Answer C}

This patient's history and CT findings are most consistent with a subdural hematoma which is caused by torn bridging veins. Patients will experience progressive neurologic deficits which if left untreated can be fatal. CT scan of the head will demonstrate a crescent-shaped lesion that may extend across the entire hemisphere. This typically occurs in older or alcoholic patients with recent head trauma, often due to a fall. Elderly patients are at increased risk due to age-related cerebral atrophy which can exert tension on the bridging veins. A family member with polycystic kidney disease (A) is often seen in patients with subarachnoid hemorrhage due to the disease's association with berry aneurysms in the Circle of Willis that are prone to rupture. Cerebral arteriovenous malformations (B) can spontaneously bleed and result in a sudden intense headache and/or loss of consciousness. A temporal bone fracture (E) and subsequent injury to the middle meningeal artery (D) can result in an epidural hematoma. This classically presents with a loss of consciousness in patients with head trauma, followed by a lucid interval, 
and then a loss of consciousness again. The hematoma is lenticular (lens shaped) or convex in shape.

\section{Answer A}

Patients with increased intracranial pressure (ICP) will benefit with therapy aimed at decreasing ICP. Hypertonic saline solution will decrease cerebral edema by acting as an osmotic force, drawing fluid out of the tissues and into the blood and thus decrease ICP. Fluids that contain hypotonic saline (such as dextrose $5 \%$ water) would have a deleterious effect (D). Mild hyperventilation to create hypocapnia is also beneficial as it induces mild vasoconstriction $\left(\mathrm{CO}_{2}\right.$ is the most potent cerebral vasodilator). Excessive hyperventilation can be deleterious as it will decrease overall perfusion. Ventilation with permissive hypercapnia (B) is a technique used in ARDS that helps prevent barotrauma. However, hypercapnia (high $\mathrm{CO}_{2}$ ) would lead to cerebral vasodilation and worsen ICP. Patients with head injury should be placed in reverse Trendelenburg (head up) position. Trendelenburg position (C) will also increase ICP by using gravity to increase cerebral blood flow. Hyperglycemia (injured brain metabolizes glucose, leading to an increase in cerebral lactate with subsequent acidosis) and nitroprusside drip (vasodilation) (E) have also been associated with increased ICP.

\section{Answer C}

Numerous guidelines exist for when to perform a non-contrast CT in the setting of head trauma. The Canadian guidelines state that a head CT is indicated following minor head trauma and more than one episode of emesis, GCS $<15$ at $2 \mathrm{~h}$ after injury, evidence of basilar skull fracture (Battle's sign, raccoon eyes), focal neurologic deficit, age $>65$, dangerous mechanism of injury (e.g., ejected out of car), and suspected open or depressed skull fracture. Although she has a normal GCS, since she has vomited twice, a CT scan is indicated. Discharging the patient (D) would be inappropriate because she meets the criteria for getting a head CT. A patient with a minor, isolated head injury and a negative CT could be safely discharged (D) with instructions given to the patient and/or family/caregiver to look for signs of increased intracranial pressure (e.g., lethargy, vomiting, intense headaches, deficits in short-term memory, seizures, or focal neurologic deficits). Patients with these signs should return to the hospital immediately. Administration of corticosteroids $(\mathrm{B}, \mathrm{E})$ is not indicated in patients with head injury.

\section{Answer $\mathrm{E}$}

Any postoperative breast cancer patient with a severe headache should be evaluated for metastasis to the brain with a CT scan of the head (A). Postoperative radiation therapy is offered in conjunction with breast-conserving therapy (e.g., lumpectomy) to appropriate patients. Complications of radiation therapy include pneumonitis, ulceration, sarcoma, con- tralateral breast cancer, and red patches of skin or "radiation burns." Skin biopsy (C) is not needed to confirm this. These can be severe and very painful or, in this patient's case, can only be a cosmetic burden. Options A and B are not typically needed to evaluate for brain metastasis. Carotid disease does not typically present with headaches so duplex scan (D) would not be indicated.

\section{Answer D}

Once the ABCs have been addressed in a patient presenting with traumatic brain injury, one can assess a patient's level of consciousness by using the Glasgow Coma Scale (GCS) as part of the primary neurologic survey. GCS is composed of three components: eye opening, verbal response, and motor response. The sum total of the three components is the GCS score, which ranges from 3 to 15 . This patient has an eye score of 3 , verbal score of 4 , and a motor score of 5 . Thus, her GCS is 12 and classified as a moderate head injury.

\section{Answer D}

Missing cervical spine injuries can have catastrophic consequences for patients. The risk of neurologic sequelae is 10 times higher in patients with missed cervical injuries during initial screening versus those with injuries identified early on. CT imaging alone (A) may be sufficient to detect all clinically meaningful injuries in patients with normal mentation. However, obtunded or unexaminable patients may have higher rates of occult ligamentous injuries with this modality alone. Obtunded patients with a negative CT scan of the neck should be further evaluated with an MRI of the neck to optimally clear them of any cervical spine injury. CT scan of the neck should always be done first $(B, C)$ in all patients being cleared from cervical spine injury, regardless of mentation. The C-spine can sometimes be cleared using physical examination (without imaging) (E). However, this is only true for patients that are awake, alert, cooperative, and without any distracting injuries.

\section{Answer B}

A solitary brain mass in the gray-white junction with surrounding edema is most consistent with a metastatic tumor. Although the most common source would be lung cancer, the history of a toe amputation highly suggests a subungual melanoma. This may occur even in patients with a remote history of melanoma. Type IV astrocytoma (glioblastoma multiforme) (A) is considered to be the most common primary malignant brain tumor in the United States. It can cross the corpus callosum, giving it a "butterfly" appearance. Oligodendroglioma $(\mathrm{C})$ is a rare, slow-growing tumor often found in the frontal lobes. Meningioma (D) is the second most common primary tumor in adults. It arises from arachnoid cells and is characterized by psammoma bodies on histologic examination. Ependymoma (E) primarily affects 
children and commonly occurs in the fourth ventricle leading to hydrocephalus. Patients have a poor prognosis.

\section{Answer D}

Patients with subarachnoid hemorrhage can have multiple complications after their initial presentation including rebleeding, hyperglycemia, acute hypoxia, and electrolyte abnormalities. Patients are at risk for symptomatic hyponatremia secondary to cerebral salt-wasting syndrome. This is thought to occur because of the inappropriate secretion of vasopressin resulting in water retention. In addition, these patients have increased levels of atrial natriuretic peptide and brain natriuretic peptide which contribute to salt wasting. Patients with hyponatremia may present with seizures, lethargy, agitation, confusion, and nausea, and if untreated, patients can eventually fall into a coma. Nephrogenic diabetes insipidus (A) is a renal dysfunction in which the collecting ducts are unresponsive to ADH, while central diabetes insipidus (B) is associated with decreased levels of ADH released from the posterior pituitary. Both of these conditions are associated with hypernatremia. Thyroid dysfunction (E) can rarely lead to loss of sodium. However, there is no evidence to believe the presented patient has any thyroid problems.

\section{Answer C}

$\mathrm{HIV}$ + patients that present with CD-4 counts between 50 and 100 per $\mathrm{mm}^{3}$, and ring-enhancing lesions found on CT scan of the head should be suspected of having either toxoplasmosis or CNS lymphoma. These are clinically indistinguishable and occur in similar frequencies. Patients should always be started first on an empiric therapeutic trial of pyrimethamine and sulfadiazine to treat a presumed toxoplasmosis infection. If this does not resolve the patient's symptoms and/or findings (e.g., ring-enhancing lesions on CT), the next best step is to perform a stereotactic brain biopsy (D) to confirm CNS lymphoma. Additionally, HIV+ patients with CNS lymphoma almost always exhibit evidence of Epstein-Barr virus (EBV) in their cerebrospinal fluid (CSF). Although no consensus exists on treatment for HIV-related CNS lymphoma, few studies have looked at treatment with radiation therapy (A) or chemotherapy (B) and have reported only modest gains. Restarting HAART in a patient suspected of having toxoplasmosis or CNS lymphoma increases the risk of developing immune reconstitution inflammatory syndrome (IRIS) (E).

\section{Answer E}

This patient is most likely presenting with an epidural abscess. Patients with previous spinal surgeries (even if remote) or a history of IV drug abuse are at higher risk. The clinical triad of epidural abscesses includes focal back pain (or headache, if abscess is located intracranial), abnormal inflammatory parameters (fever, leukocytosis, elevated ESR), and neurologic deficits (e.g., right leg weakness). MRI is the imaging modality of choice to confirm the diagnosis. Patients should be started on long-term intravenous antibiotics and undergo surgical drainage of the abscess. NSAIDs, physical therapy, and education (A, D) would be appropriate recommendations for patients with lumbar strain. These patients would not be expected to have fevers or focal neurologic deficits. Current guidelines do not recommend corticosteroids (B) after acute spinal cord injury.

\section{Answer C}

This patient's arm weakness and inability to differentiate between hot and cold in the bilateral upper extremities are most likely due to syringomyelia, which is the presence of a cystic structure in the central cervical spinal cord. This most commonly occurs from congenital conditions (e.g., Arnold Chiari malformations) but may also occur months or years after a cervical spinal injury, such as whiplash (e.g., sudden distortion of the neck associated with extension). Traumainduced syringomyelia typically involves the cervical spinal cord and results from impaired CSF drainage in the central canal of the spinal cord which may lead to a fluid-filled cavity that compresses the surrounding spinal cord. Patients will present with weakness and decreased pain and temperature sensation in bilateral upper extremities with no other sensory deficits. Spinal spondylosis occurs as a result of degenerative joint disease (e.g., osteoarthritis) and presents with radiculopathy secondary to affected nerve roots. Trauma-induced brachial plexus injuries (B) are more likely to occur immediately following the traumatic incident and will not present with an inability to differentiate between hot and cold in the upper extremities. An autoimmune condition (D), such as multiple sclerosis, will more than likely have two lesions and present in a relapsing-remitting course. A herniated disk (E) is likely but will present with radiculopathy in the affected nerve roots. Decreased sensation to hot and cold would not be expected. 


\section{Orthopedic}

\section{Areg Grigorian and Christian de Virgilio}

\section{Questions}

1. A 35-year-old female telemarketer presents with complaints of pain in her right wrist for the past 6 months. She also reports having numbness in her right hand that occurs with wrist flexion or extension. For the past month, she has been receiving corticosteroid injections after her doctor diagnosed her with carpal tunnel syndrome. However, her pain has dramatically increased over the past week and has started to affect her job performance. Her mother has rheumatoid arthritis. Her hand appears to be swollen and is tender to palpation. She is scheduled for a synovial fluid aspiration. Which of the following findings in the aspirate would indicate surgical intervention?
(A) Glucose of $90 \mathrm{mg} / \mathrm{dL}$
(B) $1.8 \times 10^{3} / \mu \mathrm{L}$ white blood cells $/ \mathrm{mcl}$
(C) High abundance of rheumatoid factor
(D) Negatively birefringent crystals
(E) Thick yellow-green fluid

2. An 11-year-old boy presents to his pediatrician because his mother is concerned that he has been limping for the past 3 days. He states that he is limping because of a dull, aching pain in the right groin and thigh that extends down to his right knee. He has no history of preceding trauma. The pain is increased by physical activity. On physical exam, there is a shift of the torso over the affected hip with standing. Which of the following explains this physical exam finding?
(A) Weakness in hip abduction
(B) Weakness in hip flexion
(C) Weakness in hip extension
(D) Weakness in knee extension
(E) Weakness in knee flexion

3. A 24-year-old woman presents to the ED with shoulder pain after a sports injury. She is found to have an anterior dislocation of the humeral head from the glenoid fossa. Which of the following is most likely to be injured in association with this type of dislocation?
(A) Musculocutaneous nerve
(B) Radial nerve
(C) Axillary nerve
(D) Brachial arterial
(E) Medial clavicle

4. A young man is doing some woodworking in his backyard when the saw blade falls off the harness and on to his hand, severing his right thumb. After raising the right arm and applying direct pressure to control bleeding, which of the following is the best recommendation to keep his digit viable for potential replantation?

(A) Immediately place the digit directly in a small bag filled with cold water

(B) Immediately place the digit directly on top of ice

(C) Remove any dirt from the digit first, and then place directly on top of ice

(D) Remove any dirt from the digit first with a clean damp cloth, and then place digit directly in cup of cold water

(E) Remove any dirt from the digit first, wrap in a clean damp cloth, put in a plastic bag, and then place bag directly in cup of cold water

5. A 20-year-old college football player sustained a knee injury following a tackle. In the ED, his knee is very swollen, and a proper examination is not possible due to pain. Plain X-rays of the knee are negative. He is discharged home with a knee brace and crutches. During follow-up 2 weeks later, the pain and swelling are significantly improved. Which of the following findings would be most consistent with a tear to the posterior cruciate ligament?

(A) The lower leg sags on passive flexion of the knee at $90^{\circ}$ while the patient is supine

(B) The lower leg moves forward relative to the knee with forward traction

(C) The foot moves laterally when the knee is pushed medially

(D) The foot moves medially when the knee is pushed laterally

(E) A clicking sensation is appreciated when the knee is flexed and the leg is rotated externally

6. A 16-year-old boy arrives for his 1 month follow-up after straining his back and fracturing his tibia during a snowboarding accident. He has a long leg cast and uses crutches to get around. He reports that he lost the crutches given to him upon discharge but has been using an old pair that belonged to his older brother. His only complaint is a weak left hand that sometimes "falls" when he stretches out his arm. On physical exam, he has sensory deficits on the lateral dorsal side of his left hand. His leg is not bothering him and appears to be healing well. What is the most likely explanation for his upper extremity abnormalities?

(A) Missed midshaft humerus fracture

(B) Radial nerve injury

(C) Long thoracic nerve injury

(D) Ulnar nerve injury

(E) Musculocutaneous nerve injury

7. A 45-year-old alcoholic male presents with fevers and right-hand pain. He cannot recall what happened but 
thinks he may have punched someone in the face in a bar fight two nights earlier. He has tried over-the-counter antiinflammatory agents, but they have not helped decrease the pain. His temperature is $100.8^{\circ} \mathrm{F}$, blood pressure is $132 / 88 \mathrm{mmHg}$, and pulse is $78 / \mathrm{min}$. On physical exam, he has a skin break over his second phalanx-metacarpal region. He is holding his second finger in slight flexion. He has a sausage-shaped swelling of the finger, as well as flexor tendon sheath tenderness that extends the entire length of the tendon. His pain increases with passive motion of the finger. What is the most likely diagnosis?
(A) Suppurative tenosynovitis due to Eikenella corrodens
(B) Gout
(C) Dupuytren's contracture
(D) Suppurative tenosynovitis due to Pasteurella multocida
(E) Felon

8. A 5-year-old boy is brought to the pediatrician because of left knee pain that is causing him to limp. His $\mathrm{PMH}$ is significant for severe asthma. He was recently hospitalized for a severe exacerbation requiring a prolonged steroid taper. On physical exam, his temperature is $98.7^{\circ} \mathrm{F}$, blood pressure is $88 / 56 \mathrm{~mm} \mathrm{Hg}$, pulse is $76 / \mathrm{min}$, and respirations are $16 / \mathrm{min}$. He has a normal knee exam. Labs are drawn and show WBC of $7.4 \times 10^{3} / \mu \mathrm{L}$ (normal $4.1-10.9 \times 10^{3} / \mu \mathrm{L}$ ) and $\mathrm{Hgb}$ of $12.7 \mathrm{~g} / \mathrm{dL}(12-15 \mathrm{~g} / \mathrm{dL})$. ESR and CRP are within normal limits. Which of the following is the most likely diagnosis?
(A) Septic arthritis
(B) Osteomyelitis
(C) Slipped capital femoral epiphysis
(D) Torn ACL
(E) Osteonecrosis of the hip

9. A college athlete presents with shoulder pain. He reports that he was injured during basketball practice 2 days ago when his teammate ran into his shoulder. On physical examination, he has an edematous left shoulder. The clinician passively abducts the patient's shoulders to $90^{\circ}$ and flexes to $30^{\circ}$ while asking the patient to point his thumbs down. What muscle is the clinician trying to evaluate?
(A) Supraspinatus
(B) Infraspinatus
(C) Teres minor
(D) Teres major
(E) Subscapularis

10. A 45-year-old Internet insurance salesman presents to his doctor with progressive pain in his left hand for 1 year. His pain is primarily limited to the left thumb and index finger. After finishing the physical examination, his doctor believes he has carpal tunnel syndrome (CTS). Which of the following did the doctor most likely find on physical examination to support this diagnosis?

(A) Percussing the wrist elicits pain in all five fingers and the palmar surface

(B) Pain felt along the left thumb, index, middle finger when placing the elbow on a table and flexing the wrist for $60 \mathrm{~s}$

(C) Pain felt along the left thumb, index, middle finger, and palmar surface when placing the elbow on a table and flexing the wrist for $60 \mathrm{~s}$

(D) Radial deviation of the wrist upon wrist flexion

(E) Posterior and lateral forearm sensory deficit

11. A 2-week-old female born at 39 weeks gestation to a 36-year-old G2P1 female via normal spontaneous vaginal delivery comes to her pediatrician for her 2-week well-baby exam. The birth was unremarkable, and the baby went home with her mother after 2 days. She received prenatal care starting at 10 weeks gestation and had a normal, healthy pregnancy with no issues. On physical exam, as the hip is gently adducted and posterior pressure is applied, there is a palpable clunk. Which of the following is the next step in management?

(A) Reexamine at 1-month exam

(B) CT scan of the hips

(C) X-ray of the hips

(D) Reassurance

(E) Ultrasound of the hips

12. A 45-year-old woman presents with pain in her right hand for 2 days. She reports falling down and attempting to break her fall with an outstretched hand. Physical examination is significant only for pain in the anatomic snuffbox of her right hand. She has no motor weakness or sensory deficits. What is the most appropriate next step in management?

(A) Radiograph of hand and, if normal, recommend supportive care with follow-up

(B) Radiograph of hand and, if normal, recommend thumb spica cast with follow-up

(C) Thumb spica cast without radiograph

(D) Supportive care without radiograph

(E) CT scan of hand

13. An 88-year-old woman is brought in by ambulance after falling in her house. She complains of right hip pain. On physical exam, her temperature is $99.1^{\circ} \mathrm{F}$, blood pressure is $110 / 75 \mathrm{mmHg}$, pulse is $110 / \mathrm{min}$, and respirations are $20 / \mathrm{min}$. The leg appears externally rotated and shortened. X-ray shows femoral neck fracture. Which of the following is a complication of femoral neck fracture? 

(A) Avascular necrosis
(B) Lumbar radiculopathy
(C) Long-term loss of hip abduction
(D) Long-term loss of hip adduction
(E) Osteosarcoma

14. A 28-year-old man presents to the ED with an inability to move his right arm. After his initial work-up and imaging is completed, he is found to have a posterior dislocation of the right shoulder. Which of the following are most commonly associated with this type of injury?
(A) Fall on outstretched hand
(B) Weight lifting
(C) While walking dog, it suddenly tugs on leash
(D) Electrocution
(E) Surfing

15. An obese 11-year-old presents to his pediatrician because his mother is concerned that he has been limping for the past day. He states that he is limping because of dull pain in his right knee. He has no history of preceding trauma. The pain is increased by physical activity. On physical exam, there is tenderness to palpation at the anterior hip along with limitation in internal rotation of the hip. Knee exam is normal. Plain films show subluxation of the right femoral head. Which of the following is the most appropriate definitive treatment?
(A) Supportive therapy with rest and ibuprofen
(B) Aspiration of the synovial fluid along with appro- priate antibiotic therapy
(C) Weight loss program
(D) Operative stabilization
(E) Pavlik harness

16. An 18-year-old soccer player presents to her doctor 2 days after getting kicked in the knee during her championship game. She reports hearing a snap immediately after the injury, but her knee looked normal. The next day, she had a swollen kneecap with progressive pain. On physical exam, she has medial joint line tenderness and effusion. Her doctor notices an audible snap occurring when extending the knee from a fully flexed position while applying tibial torsion. What is the most likely diagnosis?
(A) Collateral ligament tear
(B) Anterior cruciate ligament tear
(C) Stress fracture
(D) Tendon displacement
(E) Meniscus tear

17. A 32-year-old housekeeper comes to the physician for hip pain localized to the lateral aspect of the hip. The hip pain is interrupting her sleep. She denies any muscle weakness or numbness and tingling. The pain is not worse with physical activity. On physical exam, there is tenderness to palpation on the lateral aspect of her hip while in the lateral decubitus position. Which of the following is the most likely etiology of the hip pain?
(A) Hip osteoarthritis
(B) Meralgia paresthetica
(C) Trochanteric bursitis
(D) Osteonecrosis
(E) Hip fracture

18. A 17-year-old male comes to the emergency department for the third time over the last month due to pain in his right thigh above his knee that is particularly bothersome at night. He was previously diagnosed with growing pains. Now he has developed swelling above his right knee and states his pain is worse. On physical exam, there is a soft tissue mass that is tender to palpation. The skin overlying the mass is erythematous. An X-ray is obtained and shows a "sunburst" pattern in the distal femur. What is the most likely diagnosis?
(A) Ewing's sarcoma
(B) Osteosarcoma
(C) Osteomyelitis
(D) Osteochondroma
(E) Osteoid osteoma

19. Football players are subjected to multiple sports injuries including valgus stressing of the knee, particularly when they get tackled in the lower extremities subjecting the knee joint to increased abduction. Determining injury to the soft tissue structures of the knee may prove to be difficult for non-orthopedic or sports medicine physicians. After a physical exam, what is the most appropriate study to order to help work-up a patient with pain following valgus stressing of the knee?
(A) MRI
(B) $\mathrm{CT}$
(C) Radiograph
(D) Arthroscopy
(E) Nerve conductions studies

20. An ambitious young surgeon decided to travel to Somaliland with an international medical team to provide services for patients that have not had any previous medical attention. His work involved traveling long distances by foot and standing up for many hours while performing minor and major surgeries for an intense 3 -week period. After returning from the trip, he experiences fatigue and increasing pain upon palpation at the midpoint of his right leg that wakes him up at night. On physical exam, he has point tenderness in his proximal tibia with some surrounding edema of the skin. Plain X-ray of the tibia is negative. What is the most likely etiology for his pain? 

(A) Medial tibial stress syndrome (MTSS)
(B) Osgood-Schlatter
(C) Stress fracture
(D) Exertional compartment syndrome
(E) Osteomyelitis

21. A 25-year-old man arrives to the ED after a MVC. After primary survey and secondary survey are completed, he was found to have a right-sided pneumothorax, a fracture in the right clavicle, and a fracture of the left midshaft humerus. A bruit is heard over the right upper chest. What is the most appropriate next step after placing a chest tube and subsequent chest X-ray to confirm placement of the tube?

(A) CT angiogram of the chest and arm

(B) Non-contrast CT of the shoulder and clavicle

(C) MRI study
(D) Nerve conduction test to assess for radial nerve injury

(E) Echocardiogram

22. A 28-year-old pregnant woman suffering from preeclampsia is being monitored in the hospital the night before her elective induction when she experiences a generalized seizure. After the successful delivery of a healthy baby boy the next morning, her doctor notices that she is unable to move her right arm. An upper extremity anteroposterior radiograph is taken but does not reveal any abnormalities. What is the most appropriate next step in management?

(A) Repeat radiographic studies of the upper extremity

(B) MRI of the upper extremity

(C) CT scan of the upper extremity

(D) Operating room

(E) Reassurance and observation 


\section{Answers}

\section{Answer $E$}

This patient was recently diagnosed with carpal tunnel syndrome but presents with an acute episode of septic arthritis which can be managed with surgical washout. Any patient with an inflammatory condition that has a sudden and dramatic increase in pain should always be suspected of this and appropriately worked up with synovial fluid analysis. Surgical washout would be indicated in this patient if she had thick purulent (e.g., yellow-green) material. Furthermore, she was getting local injections of corticosteroid which is a relative indication for surgical washout given the potential immunocompromised state. Glucose $<25 \mathrm{mg} / \mathrm{dL}$ and $\mathrm{WBC}>$ $2 \times 10_{3} / \mu \mathrm{L}$ (often greater than $50 \times 10_{3} / \mu \mathrm{L}$ ), $>2,000$ (often $>50,000)$ would be suggestive of septic arthritis (A, B). Rheumatoid factor $(C)$ found in synovial fluid would be supportive of a diagnosis of rheumatoid arthritis, which is treated systemically. Finally, negative birefringent crystals (D) are the hallmark of gouty arthritis, which is not treated surgically.

\section{Answer A}

The patient is most likely exhibiting slipped capital femoral epiphysis (SCFE), which affects obese adolescent males. The physical exam finding being described is the Trendelenburg sign, which is a shift of the torso over the affected hip due to gluteus muscle weakness. In addition, physical exam may reveal that the patient's gait is altered with the patient taking a short step on the affected side to minimize weight bearing due to pain. The anterior hip may be tender to palpation. Despite a complaint of pain in the knee area, the knee examination is normal $(\mathrm{D}, \mathrm{E})$.

\section{Answer C}

Anterior dislocations of the humeral head increase the patient's risk for concurrent axillary nerve injury. All these patients should be evaluated for axillary nerve damage. This can cause paralysis of the deltoid and teres minor muscles as well as a loss of sensation over the upper lateral arm. Musculocutaneous nerve injuries (A) are not common in patients that have sports injuries. These patients will present with paralysis or weakness in the biceps and brachialis muscles. Radial nerve injuries (B) are associated with fractures of the humeral midshaft. Brachial arterial injury (D) is a concern for patients with a supracondylar fracture. Medial clavicle injury (E) is difficult to attain and is not expected to occur concurrently in patients that have an anterior dislocation of the humeral head.

\section{Answer $E$}

Severed body parts can sometimes be reattached if the proper steps to ensure tissue viability are taken. There are new and promising replantation techniques available. However, nerve regeneration continues to be a major limiting factor. Young patients with sharp amputations and no crush injury are the best candidates for replantation. The best recommendation to keep an amputated digit viable is to remove any dirt from the digit first, wrap it in a clean damp cloth, put it in a plastic bag, and then place the bag directly in a cup of cold water. This will ensure viability for up to $18 \mathrm{~h}$.

\section{Answer A}

The most consistent finding with a tear in the posterior cruciate ligament would be a lower leg that sags on passive flexion of the knee at $90^{\circ}$ while the patient is supine. An ACL tear would present with the lower leg moving forward relative to the knee with forward traction (B). A tear in the MCL would present with a foot that moves laterally when the knee is pushed medially $(\mathrm{C})$. A tear in the LCL would present with a foot moving medially when the knee is pushed laterally (D). A clicking sensation that is heard when the knee is flexed and the leg is rotated externally is known as McMurray's sign and is positive with a meniscal tear $(\mathrm{E})$.

\section{Answer B}

Radial nerve injuries present with wrist drop and sensory loss to the posterior arm and lateral dorsal aspect of the hand. This most commonly occurs in patients that have had fractures to the humeral midshaft and those that use improperly fitted crutches. This patient is using crutches that belonged to his older brother and is likely responsible for his upper extremity abnormalities. If a fracture in the midshaft of the humerus (A) did not present clinically during the time of his snowboarding accident, it is unlikely to cause significant problems a month later. Long thoracic nerve injuries $(C)$ can occur in women with breast cancer that receive axillary lymphadenectomy. This will present with scapular winging. Ulnar nerve injury (D) presents with sensory changes in the 4th and 5th digits. Musculocutaneous nerve injuries (E) will present with paralysis or weakness in the biceps and brachialis muscles.

\section{Answer $A$}

This patient has suppurative tenosynovitis with the characteristic four cardinal signs (Kanavel signs): flexor tendon sheath tenderness, fusiform swelling (sausage-shaped digits), pain with passive extension, and a semi-flexed posture of the involved digit. It is a closed space infection, and some may be associated with past penetrating injuries to the hand. The most likely organism is Eikenella corrodens which is often associated with human bites (e.g., punch in the mouth/face). Pasteurella multocida (D) can also cause suppurative tenosynovitis but is associated with cat scratches. Management of suppurative tenosynovitis involves mid-axial longitudinal incision and drainage. Gout (B) is a crystalinduced arthropathy that commonly first presents in the big toe (podagra). Dupuytren's contracture $(C)$ is associated 
with diabetes, smoking, and alcohol abuse. It presents with contractures in the fourth and fifth digits secondary to proliferation of the palmar fascia of the hand. Felon (E) is a term used to describe infection in the terminal joint space of the finger.

\section{Answer $E$}

This patient is most likely exhibiting avascular necrosis of the proximal femoral head (hip) (Legg-Calvé-Perthes disease). Avascular necrosis of the hip typically presents as hip pain, anterior thigh pain, or referred knee pain as well as a limp (which may be painless) in children between the ages of 4 and 10 years with a male-to-female ratio of $4: 1$. Often, children are unable to localize hip pain and may state they have knee pain, confusing the picture. Avascular necrosis is usually idiopathic but may occur secondary to an underlying condition such as glucocorticoid use. Clinical features include insidious onset of hip pain and limp. Physical exam shows limitation in internal rotation of the hip. Initial radiographs are often normal. Later in the course, radiographs show fragmentation of the femoral head. Septic arthritis presents with acute onset of refusal to bear weight, pain, swelling, warmth, with fever, and leukocytosis (A). Osteomyelitis is spread hematogenously in children and affects the metaphysis of long bones. It presents with localized pain and fever along with leukocytosis and elevated ESR and CRP (B). Slipped capitol femoral epiphysis (SCFE) affects obese adolescent males and presents with altered gait (C). Torn ACL affects adolescent girls more often and would present with a history of trauma, swelling at the knee joint, and increased anterior translation of the knee on physical exam (D).

\section{Answer A}

This maneuver is called the drop arm test and is used to evaluate for a tear in the supraspinatus muscle, a rotator cuff injury. This is performed by passively abducting the patient's shoulders to $90^{\circ}$ and flexing to $30^{\circ}$ while asking the patient to point his thumbs down. The test is positive if the patient is unable to keep arms elevated after the examiner releases. The supraspinatus is part of the rotator cuff, along with the infraspinatus (B), teres minor (C), and the subscapularis muscles. Teres major (D) is not part of the rotator cuff.

\section{Answer B}

Carpal tunnel syndrome is a clinical diagnosis and can be supported with a positive Tinel's and/or Phalen's sign. Tinel's sign is elicited by gently percussing over the median nerve at the carpal tunnel. A positive sign is present if the patient described an electrical shock sensation in the median nerve distribution (A). Phalen's test is performed by having the patient place their elbow on a table and flexing the wrist for $60 \mathrm{~s}$. The test is considered positive if the patient reports paresthesias in the median nerve distribution. The median nerve controls sensation to the thumb, index, middle, and half of the ring finger. Palmar sensation is not affected by carpal tunnel syndrome $(C)$ because the superficial palmar cutaneous branch of the median nerve passes superficially to the carpal tunnel. Radial deviation of the wrist upon wrist flexion (D) would be expected with ulnar nerve injuries. Posterior and lateral forearm sensory deficit (E) would be expected with musculocutaneous nerve injuries.

\section{Answer $E$}

This infant is exhibiting developmental dysplasia of the hip (DDH), which describes a spectrum of conditions that cause the abnormal development of the acetabulum and proximal femur in infants and children. In young infants, it is important to routinely evaluate for DDH using the Barlow and Ortolani maneuvers, which are physical examination techniques to detect hip instability that use adduction and posterior pressure to feel for dislocatability and abduction to feel for reducibility, respectively. If a sensation of a "clunk" is found on physical exam, further work-up is warranted including immediate referral to an orthopedic surgeon. Reassurance and reexamination would be inappropriate (A, D). Ultrasound is the primary imaging technique for assessing abnormalities of the hip until 4-6 months of age because plain X-rays have limited value in the first 6 months of life when the femoral head and acetabulum are not yet ossified (C). CT scan is not useful is the diagnosis of DDH (B). Treatment involves splinting, casting, and/or surgery.

\section{Answer B}

This patient has a classic history for a scaphoid fracture. This injury usually occurs in patients that fall on an outstretched hand with the wrist extended and presents with tenderness in the anatomic snuffbox. Plain films are typically unrevealing of scaphoid fractures if taken soon after injury. If clinical suspicion is high, all patients should be immobilized with a thumb spica cast and reimaged 7-10 days later. Supportive care with or without radiographs is not appropriate for classic cases of scaphoid fractures (A, D). Radiographs are recommended for all patients (C) to look for more serious injuries that may be associated with scaphoid fractures. If patients are found to have signs suggestive of wide displacement or ligament disruption, then more advanced imaging studies should be done (E).

\section{Answer A}

Femoral neck fractures are most commonly seen in elderly patients after a fall. Women sustain hip fractures more often due to their higher rates of osteoporosis. Femoral neck fractures have a relatively high rate of complications compared with extracapsular hip fractures because they are intracapsular. The risk for compromise in the blood supply to the 
femoral neck as a result of the fracture increases the risk of complications, in particular avascular necrosis (AVN). Long-term loss of hip abduction/adduction is prevented with physical rehabilitation (C-D). Osteosarcoma (E) or lumbar radiculopathy (B) are not complications of a femoral neck fracture.

\section{Answer D}

An anterior dislocation of the shoulder joint is the most common form of shoulder dislocation. Posterior dislocations are rare and occur most often in patients that have had generalized seizures or have been electrocuted. Patients with posterior shoulder dislocations will present with an adducted arm that is internally rotated, while anterior dislocations present with an externally rotated arm. Sport injuries (A) are more likely to cause anterior dislocations of the shoulder joint. Similarly, a sudden forceful subluxation of the shoulder that may occur from a dog tugging at the chain (C) can lead to an anterior shoulder dislocation. Weight lifters are at increased risk for thoracic outlet obstruction which presents with symptoms caused by obstruction of nerves and vessels passing from the thoracocervical region to the axilla (e.g., upper extremity paresthesia, weakness, and edema). Swimmers and surfers (E) are more likely to present with symptoms consistent with nerve impingement secondary to repetitive paddling.

\section{Answer D}

The patient is most likely exhibiting slipped capital femoral epiphysis (SCFE), which affects obese adolescent males. In SCFE, the femoral head separates from the neck and slips posteriorly, resulting in a limp and impaired internal rotation. Patients often present with knee pain, so a high index of suspicion is necessary to diagnose SCFE. The physical exam finding includes altered gait such that the patient takes a short step on the affected side to minimize weight bearing due to pain and tenderness to palpation at the anterior hip. The diagnosis of SCFE is made with X-rays, which show the classic "ice cream slipping off the cone" suggesting posterior displacement of the femoral head. Obesity seems to be the strongest risk factor for SCFE, likely due to excessive mechanical stress on the physis (growth plate). Weight loss (C) can be beneficial to the overall health of the child and will decrease the risk of SCFE in the contralateral hip, but is not considered a definitive management for SCFE. Treatment involves operative stabilization using pinning of the hip joint. Supportive therapy with rest and ibuprofen would be the appropriate treatment for transient synovitis (A). Aspiration of the synovial fluid along with appropriate antibiotic therapy would be the treatment for septic arthritis (B). Pavlik harness (E) is used in the management of developmental dysplasia of the hip (DDH) to keep the hip in a flexed and abducted position.

\section{Answer E}

This patient has a meniscal tear with a positive McMurray's sign. This maneuver helps identify a meniscal tear and is positive if there is a palpable or audible snap occurring when extending the knee from a fully flexed position while applying tibial torsion. Meniscal tears can also present concurrently with other injuries. A medially directed blow to the lateral knee (valgus stress) results in the "unhappy triad": medial meniscus tear, medial collateral ligament tear, and anterior cruciate ligament tear. Both ligamentous (A, B) and meniscal tears can produce popping sounds during the injury, but ligamentous tears have rapid swelling occurring immediately, while meniscal tears have delayed swelling occurring the next day. Stress fractures (C) do not typically occur in the patella following trauma. Instead, they occur more commonly in long bones (e.g., tibia) that are subjected to repeat stress. A patellar tendon displacement (D) will present with a patient unable to resume weight bearing, an indentation at the bottom of the kneecap, a proximally displaced patella, and hemarthrosis.

\section{Answer C}

The most common cause of unilateral hip pain in the adult is inflammation of the trochanteric bursa. It is caused by friction of the gluteus medius tendon and the tensor fascia lata over the outer femur as a result of gait impairment, trauma, or infection. Untreated, the bursal wall thickens, fibroses, and gradually loses its ability to lubricate the outer hip. Hip osteoarthritis (A) most commonly presents in patients over 40 years of age. The principal symptom associated with osteoarthritis is hip pain, which is localized in the groin and exacerbated by activity and relieved by rest. Meralgia paresthetica is compression of the lateral femoral cutaneous nerve. The nerve is susceptible to compression. The principle symptom is numbness and tingling and/or burning pain localized in the upper outer thigh (B). Osteonecrosis presents with groin pain (D). Hip fracture would present with severe pain, inability to bear weight, and intolerance to movement of the hip (E).

\section{Answer B}

Osteosarcomas are primary malignant tumors of bone. The primary presenting symptoms of osteosarcoma is localized pain that is typically present for an extended period of time and may be worse at night. On physical exam, there may be a soft tissue mass, which is frequently large and tender to palpation. Osteosarcomas have a predilection for the metaphysis of long bones and are most commonly found in the distal femur, proximal tibia, and proximal humerus. The first diagnostic test to work-up bone pain is an X-ray, which shows periosteal bone formation, lifting of the cortex, and the classic "sunburst pattern." Growing pains present with recurrent, self-limited extremity pain. In contrast to bone pain, which is 
unilateral, growing pains are bilateral. Ewing's sarcoma presents in a similar manner, but the radiographic appearance is described as "onion peel" appearance due to the periosteum being displaced by the underlying tumor causing the characteristic periosteal reaction that produces layers of reactive bone (A). Osteomyelitis presents with localized pain, fever, and leukocytosis of acute onset (C). Osteochondroma (exostosis) (D) is a benign, firm solitary bone tumor commonly occurring in teenage males. Osteoid osteoma (E) is a benign, painful growth of the diaphysis in long bones (e.g., femur, tibia). It also occurs in teenage males. The pain is characteristically worse at night but better with aspirin. On plain film, there is a central radiolucency surrounded by a sclerotic rim.

\section{Answer A}

A medially directed blow to the lateral knee (valgus stress) results in the "unhappy triad": medial meniscus tear, medial collateral ligament tear, and anterior cruciate ligament tear. The most appropriate test to order in soft tissue injuries of the knee is a MRI. This study is the most appropriate study to investigate soft tissue injuries of the knee. It is superior to CT (B) as it is better able to demonstrate ligamentous and meniscal lesions. However, its use should only be limited for patients in which the diagnosis is in question. MRI is no more accurate than the physical examination of an experienced clinician, and so referral to a sport medicine physician or orthopedic surgeon should be considered prior to ordering a MRI. Radiographic studies (C) are not typically helpful or performed for sports injuries, but they are useful in traumatic knee injuries. Arthroscopy can be used for diagnostic purposes if the MRI is equivocal or if it is abnormal and warrants subsequent surgical intervention. Although nerve injuries may accompany knee injuries, nerve conduction studies (E) are not typically ordered in work-up of soft tissue injuries of the knee.

\section{Answer C}

This surgeon most likely has a tibial stress fracture. This injury is common in patients that have a sudden change in physical activity and/or long periods of standing. His fatigue could be explained by jet lag and returning from an intense medical mission trip. Stress fractures present with pain upon palpation and some surrounding edema of the skin. After physical exam, the first step in evaluating a possible stress fracture is a plain film. It is unlikely to show up on plain films, and so clinical judgment should dictate weather to start appropriate therapy. MRI or bone scintigraphy can be considered next to further evaluate the injury. Most patients with stress fractures could be managed with supportive therapy: reduce activity to the level of pain-free functioning, over-thecounter analgesics, stretching exercises, and/or biomechanical stress-relieving measures. MTSS (A), also known as shin splints, is a common cause of exertional leg pain in athletes.
Patients with MTSS complain of vague, diffuse pain of the lower extremity, along the middle-distal tibia associated with exertion. Conversely, patients with exertional compartment syndrome $(\mathrm{C})$ will present with exercise induced pain in the lower legs that quickly disappears with rest. This occurs in college athletes and long distance runners. The exact cause is not well understood, but several leading theories exist. During exercise, blood flow to the muscle increases, and in patients with constricted compartments (e.g., hypertrophic leg muscles in college athletes), this increased blood flow may result in increased pressures that can cause pain. Osgood-Schlatter disease (B) occurs in adolescence during a time when there is increased strain on the tibial tubercle (from repetitive quadriceps contraction via the patellar tendon). Exertional pain at the knees that resolves with rest is the most common complaint. Osteomyelitis (E) is a possibility and can be ruled out with a serum ESR. However, a stress fracture is far more likely.

\section{Answer A}

Most clavicle fractures can be managed conservatively with a shoulder string or brace for 6-8 weeks. However, all patients with fractures to the clavicle should receive a careful neurovascular examination since these patients are at risk for brachial plexus and axillary/subclavian arterial injury. CT angiogram is not routinely necessary, but is indicated in the presence of a thrill or bruit around the clavicle, diminished or absent radial/brachial pulse, fracture of the first rib, large hematoma in the supraclavicular region, or mediastinum widening on plain films. A CT scan (B) of the shoulder can help estimate the intra-articular extension of the clavicular fracture in the acromioclavicular joint; however, contrast should be given to look for arterial injury. MRI (C) can help diagnose coexistent injuries of rotator cuff or intra-articular disk of the acromioclavicular joint. Although radial nerve injuries can commonly accompany midshaft humeral fractures, they are more likely to present clinically with wrist drop and sensory loss to the posterior arm and lateral dorsal aspect of the hand. Nerve conduction studies (D) are typically not needed, particularly when there are no physical exam findings suggestive of nerve damage. Blunt cardiac injury can occur in patients following MVC. This should be considered if the patient presents with chest pain and/or hemodynamic instability. Given his age, the bruit heard in his chest is unlikely to be related to underlying cardiovascular disease. For these reasons, an echocardiogram is not indicated $(\mathrm{E})$.

\section{Answer A}

Pregnant patients with preeclampsia or eclampsia that present with an inability to move the arm following a seizure are most likely suffering from a posterior shoulder dislocation. These are rare and occur most often in patients that have had 
generalized seizures or have been electrocuted. Pregnant patients suffering from eclampsia are also at risk because of its association with seizures. Regular anteroposterior radiographs will often miss the diagnosis, and so patients suspected of having a posterior dislocation should receive axillary and lateral view radiographs. The axillary view is essential for diagnosis as it can help estimate the size of the defect in the humeral head. If radiographs are equivocal, a CT scan (C) can be ordered next. MRI (B) is considered for older patients with shoulder dislocation as they are more likely to have concurrent rotator cuff injury. Reassurance is not appropriate for shoulder dislocation (E). Most patients are able to manage a shoulder dislocation with nonoperative therapy (D). 


\section{Pediatric Surgery}

\section{Paul N. Frank, Areg Grigorian, and Christian de Virgilio}

\section{Questions}

1. A 4-week-old boy presents with a 3-day history of forceful vomiting. The mother states that the vomitus only contains partially digested milk. She notes that the infant seems very hungry between feedings and drinks vigorously. Past history is significant for a skin infection for which the infant received oral erythromycin. On examination, the infant appears to be healthy appearing and in no acute distress. The physician feels there may be a small palpable mass in the right upper quadrant, but is not certain. Electrolytes are normal. What is the best way to establish the most likely diagnosis?
(A) Plain abdominal x-rays
(B) Laparoscopy
(C) CT of the abdomen
(D) Upper GI study with contrast
(E) Ultrasound

2. A 2-day-old male infant was diagnosed prenatally with Down syndrome. Delivery was uneventful, but pregnancy was complicated by polyhydramnios. The infant has had several episodes of bilious vomiting after breast feeding but is otherwise stable. Which of the following would be the most likely finding on further work-up?

(A) Dilated loops of small bowel with air-fluid levels on abdominal x-ray

(B) Inability to pass a nasogastric tube

(C) Abdominal distention with erythema of the overlying skin

(D) Two large air bubbles on abdominal x-ray

(E) Narrowing of the distal rectum on GI contrast study

3. A 12-day-old male born at 33 weeks becomes lethargic and hypothermic over the course of $24 \mathrm{~h}$. He is not tolerating his formula feeds, has two episodes of bilious emesis, and has three episodes of bloody diarrhea. Physical exam reveals abdominal distention, visible loops of bowel, abdominal wall erythema, and absent bowel sounds. What is the most likely diagnosis?
(A) Hirschsprung's disease
(B) Duodenal atresia
(C) Esophageal atresia with tracheoesophageal fistula
(D) Necrotizing enterocolitis
(E) Meconium ileus

4. A newborn female infant is born to a 19-year-old G1P0 mother who smokes. On physical exam, the small bowel is eviscerated through an abdominal wall defect to the right of the umbilicus. The small bowel appears matted and dilated. The infant appears to otherwise be healthy. Which of the following would have been expected in prenatal screening?
(A) Elevated b-hCG levels
(B) Decreased estradiol levels
(C) Elevated alpha-fetoprotein levels
(D) Oligohydramnios
(E) None of the above

5. A 4-month-old baby girl is seen at her pediatrician's office for her well-child check. The parents raise a concern that she has been vomiting approximately $1 / 3$ of her meals since 2 weeks of age. The emesis is the color of milk and is not bile-stained. There has been no change in the frequency or amount of emesis. She is exclusively breast-fed. On physical exam, mucous membranes are moist and the anterior fontanelle is open and flat. Her growth is at the 75th percentile for height and weight and has not changed significantly since birth. She is otherwise asymptomatic and without findings on physical examination. Which of the following is the most likely diagnosis?
(A) Tracheoesophageal fistula
(B) Duodenal atresia
(C) Pyloric stenosis
(D) Gastroesophageal reflux
(E) Malrotation

6. A 6-h-old male infant is noted to be dyspneic with an oxygen saturation of $86 \%$. Physical exam reveals subcostal retractions and moderate perioral cyanosis. Which finding on chest $\mathrm{x}$-ray would be most suggestive of a nonsurgical diagnosis?
(A) Loops of bowel in the left chest
(B) Tip of orogastric tube located above carina
(C) Diffuse pulmonary interstitial edema
(D) Boot-shaped heart with upturned apex
(E) Double-bubble sign just beneath the diaphragm

7. A newborn full-term infant is noted to have several episodes of bilious emesis. He is otherwise stable. Which of the following is the LEAST likely diagnosis?
(A) Pyloric stenosis
(B) Duodenal atresia
(C) Midgut volvulus
(D) Hirschsprung's disease
(E) Jejunal atresia

8. A 5-week-old boy presents with a 6-day history of vomiting. The parents report that his vomiting is more forceful than his usual spit ups and contain significantly more volume. The mother describes the vomit as partially digested milk. Between episodes of vomiting, the baby feeds vigorously. The parents report that he has made only one wet 
diaper today. On physical exam, the baby appears lethargic. Mucous membranes are dry and the anterior fontanelle is open and sunken. Capillary refill is $2 \mathrm{~s}$. The growth chart reveals a 0.5 -lb weight loss since his clinic visit 7 days ago. What is the next step in management?
(A) Surgical intervention
(B) CT of the abdomen
(C) Upper GI contrast study
(D) Fluid resuscitation
(E) Ultrasound

9. A 2-h-old male infant born at 39 weeks gestation is noted to be drooling. Prenatal ultrasound demonstrated polyhydramnios. Attempts at placement of an orogastric tube are unsuccessful as the tube only passes about $10 \mathrm{~cm}$ from the lips. What is the most important immediate concern for an infant with this condition?
(A) Prevention of aspiration
(B) Nutrition
(C) Urgent surgical exploration
(D) Establishing positive pressure ventilation
(E) Confirmatory contrast esophagram

10. Which of the following is the next best step in the management of a 1-week-old infant born at full term with bilious emesis?
(A) Abdominal ultrasound
(B) Broad-spectrum antibiotics and blood cultures
(C) Immediate operative repair
(D) Contrast enema
(E) IV fluids and nasogastric tube placement

11. A newborn male is found to have the majority of his small bowel eviscerated through an abdominal wall defect. The umbilicus appears to be intact. There is no membrane covering the bowel. The most important immediate risk to an infant with this condition is related to:
(A) Sepsis
(B) Respiratory compromise
(C) Cardiac anomalies
(D) Dehydration
(E) Urinary obstruction

12. A 6-week-old boy presents with a 6-day history of nonbilious, forceful vomiting. Between episodes of vomiting, the baby feeds vigorously. On physical exam, the baby has dry mucous membranes with a sunken anterior fontanelle. Capillary refill is $2 \mathrm{~s}$. He is otherwise asymptomatic without findings on physical exam. Ultrasound is obtained and is shown below. What electrolyte abnormality would you expect?

(A) Hypochloric metabolic alkalosis

(B) Hyperkalemic metabolic acidosis
(C) Hyponatremic metabolism acidosis

(D) Hyperkalemic metabolic alkalosis

(E) Hyponatremic metabolic alkalosis

13. A 2-h-old male born at 39 weeks gestation is noted to have diffi culty breathing. Which of the following would most strongly suggest a diagnosis of esophageal atresia with a tracheoesophageal fi stula?

(A) Worsening respiratory status with feeding

(B) Olive-shaped mass palpable in the epigastric region

(C) Improvement of respiratory status over the $24 \mathrm{~h}$ after birth

(D) Chest x-ray showing dilated loops of small bowel in the left hemithorax

(E) Scaphoid abdomen

14. A 2-h-old male born at 39 weeks gestation with difficulty breathing is diagnosed with esophageal atresia with a tracheoesophageal fistula and undergoes surgical repair, which was uncomplicated. Ten years later, the patient develops difficulty swallowing and often vomits undigested food shortly after eating. Which of the following is the most likely explanation for this?
(A) Scleroderma
(B) Gastroesophageal reflux
(C) Esophageal cancer
(D) Esophageal leak
(E) Esophageal stricture

15. A 2-week-old male infant born at 26 weeks gestation is in the neonatal ICU when he becomes hypotensive and begins passing bloody stools. Which of the following is the most likely finding?

(A) Palpable olive-shaped mass in the epigastric region

(B) Double-bubble sign on abdominal x-ray

(C) Loops of intestine in the left hemithorax on chest $\mathrm{x}$-ray

(D) Gas in the walls of the intestine

(E) Donut sign on abdominal ultrasound

16. Which of the following ventilator settings or respiratory measurements would be LEAST acceptable for a newborn with congenital diaphragmatic hernia $(\mathrm{CDH})$ ?

(A) $100 \% \mathrm{FiO}_{2}$

(B) PEEP of $20 \mathrm{~cm} \mathrm{H}_{2} \mathrm{O}$

(C) Inhaled nitric oxide (NO)

(D) $\mathrm{PaCO}_{2}$ of $55 \mathrm{mmHg}$

(E) $\mathrm{PaO}_{2}$ of $65 \mathrm{mmHg}$

17. A male infant is born via normal spontaneous vaginal delivery at 39 weeks gestation to a 32 -year-old G1P1. She had good prenatal care. The infant is heard grunting while trying to breathe, and he shows bilateral subcostal and intercostal retractions. The patient is observed 
closely for several hours following delivery, and his respiratory status improves over that time. Which of the following is the most likely explanation of this patient's course?
(A) Spontaneous reduction of congenital diaphragmatic hernia
(B) Closure of the ductus arteriosus
(C) Resorption of excess pulmonary fluid
(D) Paramyxovirus infection
(E) Closure of the foramen ovale

18. A 34-year-old pregnant female at 36 weeks gestation undergoes ultrasound at her obstetrician's office. The ultrasound reveals polyhydramnios. Which of the following is the LEAST likely diagnosis?
(A) Duodenal atresia
(B) Esophageal atresia
(C) Maternal diabetes
(D) Fetal posterior urethral valve
(E) Fetal anencephaly

19. A 2-week-old infant delivered at 35 weeks gestation is brought to the pediatrician by his mother who reports that he has had a harsh, barking cough and makes a highpitched whistling sound when he inhales. He has been feeding poorly but has not had a fever. The mother also says that the patient has bouts of blue discoloration around his lips, more frequently when he is lying on his back than on his stomach. Chest X-ray is normal. What is the most likely diagnosis?
(A) Foreign body aspiration
(B) Asthma
(C) Transient tachypnea of the newborn
(D) Tracheomalacia
(E) Congenital diaphragmatic hernia

20. A 1-week-old male presents with intolerance of breastfeedings as well as several episodes of bilious vomiting. On physical exam, the patient's blood pressure and temperature are normal. The infant appears to be in pain. However, the abdomen does not appear to be distended, and there is no tenderness to palpation. Plain abdominal $\mathrm{x}$-ray shows an absence of gas within the bowel, but is otherwise unremarkable. A complete blood count and electrolytes are normal. What is the next step in the management?
(A) Admit for observation
(B) Discharge patient and offer parents reassurance
(C) Exploratory laparotomy
(D) Upper GI study with oral contrast
(E) Abdominal ultrasound 


\section{Answers}

\section{Answer A}

Worsening respiratory status with feeding is suggestive of an anatomic or physiologic defect of the upper aerodigestive tract. The next step is to attempt to place an orogastric tube (OGT) and perform AP and lateral chest x-rays. Failure to pass an OGT with radiologic confirmation that the tube is in the upper esophagus is suggestive of esophageal atresia. An olive-shaped mass palpable in the epigastrium (B) suggests a diagnosis of pyloric stenosis. Dyspnea that resolves over the first $24 \mathrm{~h}$ of life (C), especially in a full-term neonate who is otherwise healthy, is likely transient tachypnea of the newborn, which is benign and self-limited. Loops of small bowel in the left hemithorax (D) suggest a congenital diaphragmatic hernia, as does a scaphoid abdomen (E) (it indicates that bowel contents are elsewhere such as in the chest).

\section{Answer D}

Given the history of polyhydramnios, bilious emesis, and Down syndrome, the most likely diagnosis is duodenal atresia. Because of the duodenal obstruction, there would be no gas in the small bowel. Air-fluid levels on abdominal x-ray (A) are characteristic of more distal intestinal obstruction. Inability to pass a nasogastric tube (B) is suggestive of choanal atresia. Abdominal distention with erythema of the overlying skin (C) is concerning for necrotizing enterocolitis. Narrowing of the distal rectum on GI contrast study (E) is found in Hirschsprung's disease.

\section{Answer D}

In a premature neonate with rather sudden systemic illness, feeding intolerance, and bloody stools, necrotizing enterocolitis would be the most likely diagnosis. Visible loops of distended bowel and abdominal wall erythema are additional classic findings. Initial treatment is to place the infant NPO, stomach decompression, and administration of IV antibiotics. Surgical management is indicated for suspected perforation, as evidenced by free intraperitoneal air or progressive clinical deterioration (rising WBC count, falling platelet count, worsening acidosis). Hirschsprung's disease (A) would present with failure to pass stool at birth and not a sudden decompensation. Duodenal atresia (B) presents with bilious vomiting, but due to the atresia, it presents at birth and with failure to pass meconium. Esophageal atresia with tracheoesophageal fistula (D) would present with respiratory distress during feeding. Meconium ileus (E) classically would present with failure to pass meconium at birth and raises concern for cystic fibrosis.

\section{Answer $\mathrm{C}$}

Eviscerated bowel without a membrane covering it, with the abdominal wall defect to the right of the umbilicus, is termed gastroschisis. It is more common in young mothers and in those who smoke during pregnancy. Maternal serum alphafetoprotein (AFP) tends to be elevated in cases of abdominal wall defects, including both gastroschisis and omphalocele (evisceration though the umbilicus and with a membrane covering bowel). Typically, maternal serum AFP is greater in gastroschisis than in omphalocele. Maternal serum AFP is checked as part of the triple screen or quad screen that is performed. Elevated maternal serum AFP is seen in other conditions such as multiple gestation, neural tube defects, abruptio placentae, or endodermal sinus tumor, making this a nonspecific marker for abdominal wall defects. Gastroschisis is associated with intestinal atresia. Since the fetus may be unable to swallow amniotic fluid, it is associated with polyhydramnios.

\section{Answer D}

Gastroesophageal reflux is a common complaint in infants less than 1 year of age. In the first year of age, the pylorus is not fully developed and therefore not fully functional, leading to occasional episodes of reflux (also called spitting up). Gastroesophageal reflux disease (GERD) is not diagnosed until the infant is failing to gain weight. Treatment of GERD includes thickening the feeds, keeping the infant upright after feeds, and feeding smaller amounts at shorter intervals. Tracheoesophageal fistula typically presents at birth with inability to control secretions. A rare " $\mathrm{H}$ " type (fistula without esophageal atresia) often presents in a delayed fashion but would present with recurrent respiratory infections from aspiration and not vomiting (A). Duodenal atresia would present as bilious vomiting in the newborn period and the classic "double-bubble" sign on abdominal x-ray (B). Pyloric stenosis presents with non-bilious, projectile vomiting in the first few weeks of life and typically not at 4 months of age (C). In addition, it would progressively worsen with time. Malrotation would present with abdominal distension and bilious vomiting $(\mathrm{E})$.

\section{Answer C}

Diffuse pulmonary interstitial and/or alveolar edema suggests transient tachypnea of the newborn, which is self-limited and resolves within 1-2 days. Loops of bowel in the left chest (A) suggest a congenital diaphragmatic hernia. An orogastric tube that does not pass beyond the proximal esophagus (B) suggests an esophageal atresia. Boot-shaped heart with upturned apex (D) is found in infants with tetralogy of Fallot, a constellation of congenital cardiac anomalies, that specifically includes ventricular septal defect, pulmonary stenosis, large overriding aorta, and right ventricular hypertrophy. The double-bubble sign $(\mathrm{E})$ is found in duodenal atresia.

\section{Answer A}

Bilious vomiting in the neonate should be considered a surgical emergency until proven otherwise. It implies obstruction 
that is distal to the ampulla of Vater and likely from a congenital anomaly (B-E). Non-bilious emesis (of milk) is more likely to be physiologic (immaturity of the lower esophageal sphincter). An exception would be non-bilious vomiting that progressively worsens and is projectile in nature, which would raise suspicion for pyloric stenosis. Since the obstruction is at the pylorus, it does not allow for gastric contents to mix with bile. Pyloric stenosis also presents later, typically in the 3rd week of life.

\section{Answer D}

The presentation is classic for pyloric stenosis. Although the management of pyloric stenosis is surgical, the first priority in these infants is rehydration. The infant is presenting with signs of moderate to severe dehydration including dry mucous membranes, a sunken fontanelle, delayed capillary refill, and decrease urinary output. The course warrants fluid resuscitation prior to any diagnostic work-up or consultations. After fluid resuscitation, the gold standard imaging modality is ultrasound to assess for pyloric stenosis (E). Only if ultrasound is negative or equivocal is an upper GI obtained (C). If imaging modalities demonstrate pyloric stenosis, surgical consultation is then obtained (A). CT of the abdomen is not warranted in the work-up of suspected pyloric stenosis (B).

\section{Answer A}

Based on the classic history of polyhydramnios and excessive drooling in a newborn, the patient likely has esophageal atresia. The most important concern is prevention of aspiration. Nutrition (B) can be established after early surgical repair or via a gastrostomy tube if surgery is not undertaken (as in cases where the infant is premature or has pneumonia from aspiration). Urgent surgical exploration (C) is not indicated, as surgery may be delayed in some patients. Positive pressure ventilation (D) may distend the stomach and cause aspiration. Therefore, it should be avoided when possible. Contrast esophagram (E) is only performed if chest $\mathrm{X}$-ray is nondiagnostic or if the location of the fistula cannot be identified. This study carries risk of aspiration pneumonitis from the contrast agent.

\section{Answer $E$}

The first steps in treating a patient with bilious emesis are fluid resuscitation and gastrointestinal decompression (via NG tube). Once the IV fluid resuscitation has begun, the patient may undergo an upper GI study to evaluate for evidence of midgut volvulus, which may present with distended proximal bowel and a paucity of gas in the distal bowel. Broad-spectrum antibiotics (B) are not indicated as there is no evidence of infection. Operative repair $(C)$ cannot occur until the patient has been stabilized and a diagnosis has been confirmed. Contrast enema (D) would be used to evaluate for
Hirschsprung's disease, which would present as failure to pass meconium and not necessarily bilious emesis.

\section{Answer D}

This patient has gastroschisis. Since the intestines are outside of the abdominal cavity, insensible fluid losses will be much greater than in an infant without gastroschisis. Therefore, covering of the exposed intestine with moist gauze and IV fluid resuscitation are critical first steps in management. Such patients are also at risk of hypothermia. While exposed intra-abdominal contents increases risk of infection and sepsis (A), this is not as immediate of a concern as is dehydration. After operative repair, patients may be paralyzed to allow the abdominal wall to relax and stretch to accommodate the intestines. If the abdominal cavity is not sufficiently large to accommodate the bowel, the bowel is covered with a silo temporarily. Attempting to forcefully reduce all the small bowels and close it under tension will result in abdominal compartment syndrome, bowel ischemia, and respiratory compromise (B). Cardiac anomalies (C) are more of a concern in patients with omphalocele than gastroschisis, but regardless this is not an immediate concern. Urinary obstruction (E) is not a typical feature of gastroschisis, though it could rarely occur if the bladder were also herniated through the abdominal wall.

\section{Answer $A$}

Laboratory evaluation in a patient with pyloric stenosis classically shows a hypochloric metabolic alkalosis. Chloride is typically lost in the gastric secretions $(\mathrm{HCl})$ via vomiting. Alkalosis is caused by both a loss of protons $(\mathrm{HCl})$ in the gastric fluid as well as secondary to a contraction alkalosis mediated by aldosterone secretion in the setting of hypovolemia. Hypokalemia is a late finding seen in infants who have been vomiting for prolonged period of time, also from contraction alkalosis. The infants also may have a paradoxical aciduria (acidic urine despite alkalosis). Initially, $\mathrm{Na}^{+}$in the renal tubule is reabsorbed in exchange for $\mathrm{K}^{+}$ions but as $\mathrm{K}^{+}$ levels decrease, $\mathrm{Na}^{+}$is instead exchanged for $\mathrm{H}^{+}$ions. The presentation of adrenal crisis in an infant may mimic that of pyloric stenosis. However, infants with adrenal crisis typically have hyperkalemic acidosis (D) rather than the hypokalemic alkalosis that is typical of pyloric stenosis (B).

\section{Answer $E$}

Ultrasound is the image modality of choice in diagnosing pyloric stenosis, as it does not require any radiation exposure. If ultrasound is negative or equivocal and pyloric stenosis is highly suspected, diagnosis may be attempted with a barium upper GI study with contrast. Upper GI was the test of choice prior to the advent of ultrasound. However, it must be done carefully as it risks causing aspiration given that the infant has a gastric outlet obstruction. Typical findings 
include elongated pyloric canal (string sign) and delayed gastric emptying. In addition, upper GI studies are further helpful in the setting of a negative ultrasound in order to assess for other items on the differential diagnosis, particularly gastroesophageal reflux. Reassurance would be inappropriate if pyloric stenosis is highly suspected (A). Operative intervention with pylorotomy would be the definitive treatment should the child be diagnosed with pyloric stenosis, but confirmatory testing should be undertaken first (B). CT of the abdomen is not an imaging modality of choice for diagnosing pyloric stenosis $(\mathrm{C})$.

\section{Answer $E$}

Esophageal anastomotic stricture is a very common longterm complication of esophageal atresia with or without tracheoesophageal fistula repair. These cases may be treated with esophagoscopy with balloon dilation. There is no evidence given to suggest the patient has scleroderma (A), which is particularly unusual to present at age 10 . The patient likely does have gastroesophageal reflux (B), as this is another typical side effect of surgical repair, but the symptoms described are more likely attributable to esophageal stricture. Esophageal leak (D) is more commonly a shortterm complication, and many leaks will heal spontaneously. Finally, the patient is at increased risk for esophageal cancer (C), but this would be unlikely to develop so early in life.

\section{Answer D}

This patient likely has necrotizing enterocolitis, which most commonly affects premature infants. It causes necrosis of segments of intestine. In the necrotic segments, gas may be found within the walls of the intestine, a finding known as pneumatosis intestinalis. A palpable olive-shaped mass in the epigastric region (A) suggests pyloric stenosis, which would present with non-bilious emesis, but not severe systemic illness. Double-bubble sign on abdominal x-ray (B) suggests duodenal atresia, which would also not present so acutely. Loops of intestine in the left hemithorax on chest $\mathrm{x}$-ray (C) suggest congenital diaphragmatic hernia, which may present with respiratory difficulty. Donut sign on abdominal ultrasound (E) arises from intussusception of intestine, wherein one segment telescopes into another. This may occur at the ileocecal junction or at the site of a diverticulum and is a surgical emergency. However, it would not necessarily present as acute systemic illness.

\section{Answer B}

Positive end expiratory pressure (PEEP) is designed to keep alveoli open throughout the respiratory cycle, even during expiration. This increases the surface area available for gas exchange. However, high levels of PEEP will increase the pressure within the alveoli to dangerously high levels and may cause barotrauma, analogous to inflating a balloon until it nearly bursts. Therefore, PEEP should be maintained at or below $5 \mathrm{~cm} \mathrm{H}_{2} \mathrm{O}$. $\mathrm{FiO}_{2}$ of $100 \%$ (A) will maximize oxygenation. Inhaled NO $(\mathrm{C})$ will cause pulmonary vasodilation, thereby reducing pulmonary hypertension. It has been shown that permissive hypercapnia, with $\mathrm{PaCO}_{2}<60 \mathrm{mmHg}$ (D), and permissive hypoxia, with $\mathrm{PaO}_{2}>60 \mathrm{mmHg}(\mathrm{E})$, allow for lower ventilation settings and less risk of barotrauma.

\section{Answer $C$}

The patient described above is an otherwise healthy, fullterm newborn whose mother had good prenatal care. Furthermore, his respiratory status improved in the hours after delivery. Therefore, the most likely diagnosis is transient tachypnea of the newborn, which resolves as excess pulmonary fluid is resorbed. Spontaneous reduction of congenital diaphragmatic hernia $(\mathrm{A})$ is very unlikely. Closure of the ductus arteriosus (B) or foramen ovale (E) will have no effect on respiratory status in healthy patients. In patients with some cyanotic heart lesions, such as transposition of the great arteries (wherein the right ventricle ejects into the aorta and the left ventricle ejects into the pulmonary artery), closure of the ductus arteriosus or foramen ovale will have an adverse effect on blood oxygenation. Paramyxovirus (D) can lead to croup, which can lead to respiratory difficulty, but this diagnosis is unlikely given the patient's presentation.

\section{Answer D}

Fetal posterior urethral valve prevents the fetus from passing urine via the urogenital tract, which reduces the amount of amniotic fluid present, and is referred to as oligohydramnios. Renal anomalies generally lead to oligohydramnios, whereas intestinal atresias are associated with excess amniotic fluid (polyhydramnios), as the fetus in unable to swallow amniotic fluid. The other items listed above are associated with excess of amniotic fluid.

\section{Answer D}

Softness of the tracheal cartilage is known as tracheomalacia. Because the cartilaginous support is soft and flexible, the airway can collapse. The condition is usually worse when the patient is supine because gravity pulls the anterior trachea downward toward the posterior trachea, thereby occluding the upper airway. Foreign body aspiration (A) (such as a toy or food particles) would present similarly, but this is unlikely in a 2-week-old. Asthma (B) is also a consideration, but tracheomalacia should be ruled out first. Transient tachypnea of the newborn (C) usually resolves within 1-2 days after delivery. Congenital diaphragmatic hernia (E) generally presents at birth. Delayed presentations rarely do occur, but the chest $\mathrm{x}$-ray would show loops of bowel, or part of the liver, in the thorax. 


\section{Answer D}

Bilious vomiting in an infant should always raise suspicion for midgut volvulus (a complication of malrotation) which left untreated can lead to intestinal gangrene. Yet, early on, ischemic bowel provides few clinical clues to the impending catastrophe, as the physical exam may be benign, with no fever, no abdominal tenderness (as only the visceral peritoneum is initially affected), and normal laboratory values (there are no values that are diagnostic of bowel ischemia). Similarly, plain abdominal x-ray may be normal. By the time the infant has evidence of systemic inflammation, peritonitis, appears toxic, or has marked leukocytosis, there is a high likelihood that there is gangrenous bowel. The gangrene may involve the entire small bowel. If such a patient survives, he/she may be subject to lifelong intravenous parenteral nutrition or may require small bowel transplantation. Thus further work-up is always required to rule out this potentially devastating problem (A-B). Given the paucity of findings, it would be premature to take this infant directly to the operating room (C). Ultrasound (D) is useful for pyloric stenosis (non-bilious vomiting) but not for midgut volvulus. Upper GI study with oral contrast is the best test as it will confirm failure of passage of the contrast, confirming a bowel obstruction, or show a malrotation. A normal study should demonstrate the normal $\mathrm{C}$ loop of the duodenum and show that the duodenal jejunal junction is to the left of the spine. 


\section{Skin}

\section{Areg Grigorian, Paul N. Frank, and Christian de Virgilio}

\section{Questions}

1. A 15-year-old girl develops short gut syndrome following resection of bowel secondary to leiomyosarcoma in the small intestinal wall. She is subsequently placed on long-term total parenteral nutrition (TPN) and is recovering well. A month later, she develops red and inflamed patches of dry and scaly skin around her mouth and eyes. Her hair also begins to thin, and she notices a bad taste when she gets her daily cherry-flavored chloraseptic spray to prevent dry throat. What is the most likely underlying etiology of her skin lesions and thinning hair?
(A) Zinc deficiency
(B) Copper deficiency
(C) Pemphigus vulgaris
(D) Chromium deficiency
(E) Psoriasis

2. A 30-year-old female of Scottish descent presents with a nodule on her face near the corner of her eye. The lesion measures $12 \mathrm{~mm}$ in diameter. The borders are irregular, and the center of the lesion is dark. Which of the following is the best recommendation?
(A) Shave biopsy
(B) Punch biopsy
(C) Excisional biopsy with $1 \mathrm{~mm}$ margin
(D) Excisional biopsy with $5 \mathrm{~mm}$ margin
(E) Reexamination in 2 months

3. A 64-year-old man who emigrated from Japan arrives to his doctor to discuss new skin lesions. His wife first noticed two discolored plaques on his back 2 weeks ago, but he now has multiple lesions all over his back, chest, and face. They are the size of a coin and appear to have a "stuck-on" appearance. He is afebrile, blood pressure is $136 / 86 \mathrm{mmHg}$, and he has a pulse of $90 / \mathrm{min}$. The skin lesions do not itch, and they are not tender. He has no other complaints, and a review of systems is negative. $\mathrm{He}$ is more concerned about his cosmetic appearance. What is the best next step in management?
(A) Reexamine in 2 weeks
(B) Skin biopsy
(C) Abdominal CT scan
(D) Mohs procedure
(E) Corticosteroids

4. Which of the following would be best suited for Mohs surgery?

(A) Superficial spreading melanoma in the arm

(B) Nodular melanoma on the back
(C) Basal cell carcinoma on the face

(D) Subungual melanoma

(E) Squamous cell carcinoma of the neck

5. A 25-year-old female lifeguard presents to her doctor to discuss a new $10 \mathrm{~mm}$ skin lesion that she found on her right forearm that has been growing over the last month. The lesion has a heterogeneous dark blue color, is symmetric, and has been growing vertically. What is the most likely diagnosis?
(A) Impetigo
(B) Melanoma
(C) Nevus
(D) Molluscum contagiosum
(E) Squamous cell carcinoma

6. A 65-year-old obese male with diabetes and a history of IV drug abuse presents with a painful swollen left leg. Exam reveals dark purple discoloration and several large bullae over the calf. Vitals are temperature of $101.1^{\circ} \mathrm{F}$, heart rate of $120 / \mathrm{min}$, and blood pressure of $92 / 68 \mathrm{mmHg}$. The CRP is $200 \mathrm{mg} / \mathrm{L}$ (normal $<10 \mathrm{mg} / \mathrm{L}$ ), and the WBC is $28.3 / \mathrm{mm}^{3}$ (normal $4-10 / \mathrm{mm}^{3}$ ). Creatinine is $2.0 \mathrm{mg} / \mathrm{dL}$ (normal $0.5-1.5 \mathrm{mg} / \mathrm{dL}$ ), and $\mathrm{Na}$ is $127 \mathrm{mEq} / \mathrm{L}$ (normal $135-145 \mathrm{mEq} / \mathrm{L}$ ). Distal pedal pulses are 1+. IV fluids and intravenous antibiotics are administered. Which of the following is the next best step?
(A) X-ray of the leg
(B) CT scan of the leg with IV contrast
(C) Venous Duplex scan of the left leg
(D) Measure compartment pressures
(E) Emergent wide surgical debridement

7. A 50-year-old field worker arrives to a free clinic to discuss a "sore" on his lower lip. He has had no trauma to the face. He reports that he first noticed the "sore" 6 months ago, and it has slowly gotten bigger. On physical exam, he has an ulcerated $1 \mathrm{~cm}$ nodule on his lower lip. There are no telangiectasias present. What is the most likely diagnosis?
(A) Basal cell carcinoma
(B) Squamous cell carcinoma
(C) Lichen planus
(D) Dermatitis herpetiformis
(E) Melanoma

8. Which of the following melanomas have the worst prognosis?
(A) Superficial spreading
(B) Nodular
(C) Lentigo maligna
(D) Acral lentiginous
(E) Subungual 
9. Which of the following melanomas do not follow the ABCDE mnemonic?
(A) Superficial spreading
(B) Nodular
(C) Amelanotic
(D) Acral lentiginous
(E) Amelanotic and nodular

10. One day following extensive debridement of the right leg for a necrotizing soft tissue infection (NSTI), a 40-year-old male remains in the ICU, intubated, and requiring $70 \% \mathrm{FIO}_{2}$. White blood cell count has risen from a preoperative level of $16 \times 10^{3} / \mu \mathrm{L}$ (normal 4.1 $10.9 \times 10^{3} / \mu \mathrm{L}$ ) to $34 \times 10^{3} / \mu \mathrm{L}$. Serum lactate has also risen. Which of the following is the best next step in treatment?
(A) Second-look operation
(B) Amputation of the right leg
(C) Broaden antibiotic coverage
(D) CT scan of the leg
(E) Start pressors

11. A 45-year-old female presents with a recent change in a preexisting mole on her anterior thigh. She states that the mole keeps bleeding, is darker, and has grown. The mole is $8 \mathrm{~mm}$ in diameter on physical exam. There are no palpable nodes in the groin. An excisional biopsy is performed with a $1 \mathrm{~mm}$ margin, and to a depth of the subcutaneous fat. Pathology reveals a melanoma that is $0.5 \mathrm{~mm}$ in thickness. The margins are negative. What is the next step in the management?
(A) No further treatment
(B) Re-excision with $1 \mathrm{~cm}$ margins
(C) Interferon alpha
(D) Granulocyte-macrophage colony-stimulating factor (GM-CSF)
(E) Dacarbazine

12. Which of the following is the most common precancerous skin lesion?
(A) Actinic keratosis
(B) Seborrheic dermatitis
(C) Seborrheic keratosis
(D) Compound nevi
(E) Keratoacanthoma

13. Where are melanomas in patients with dark skin most likely to occur?
(A) Back
(B) Arms
(C) Legs
(D) Palms, soles, and mucous membrane
(E) Face 


\section{Answers}

\section{Answer $A$}

Zinc deficiency can occur in surgical patients on long-term total parenteral nutrition or in patients diagnosed with a malabsorption syndrome. This can present with alopecia, red and inflamed patches of dry and scaly skin around the mouth and eyes, abnormal taste, and impaired wound healing. Zinc supplementation will remedy this condition. Copper (B) and chromium (D) deficiency are rare but can also affect this patient population. The most common manifestations of copper deficiency include hematologic abnormalities (anemia, leukopenia) and myeloneuropathy. Chromium deficiency presents with impaired glucose tolerance and peripheral neuropathy. Pemphigus vulgaris $(\mathrm{C})$ occurs as a result of autoimmune destruction of desmosomes between keratinocytes and is characterized by multiple skin and oral mucosa bullae. Psoriasis (E) is believed to have an autoimmune etiology and presents as salmon-colored plaques with a silvery scale that occur on extensor surfaces (e.g., patella).

\section{Answer B}

The lesion is concerning for melanoma and as such will require tissue confirmation to rule out cancer. Excisional biopsy (removing the entire lesion) (C), down to the subcutaneous fat, would be the preferred approach for a lesion on an extremity or torso. However, depending on the size of the lesion and its location (not desirable to make a cosmetically unappealing large incision if the lesion ends up being benign), an initial incisional biopsy (taking only a small sample) is preferred. Punch biopsy down through the dermis (to calculate Breslow thickness) is the preferred method in this setting. Shave biopsies (A) are not recommended if melanoma is suspected as the true Breslow thickness may not be measurable. During the initial biopsy, no attempts are made to achieve a wide margin. If the pathology comes back benign, no further treatment may be necessary. Excisional biopsy with a $5 \mathrm{~mm}$ initial margin (D) would not be indicated as the lesion may be benign. Reexamination (E) is not appropriate for a patient suspected of having melanoma.

\section{Answer C}

The skin lesions described are most likely to be seborrheic keratosis (SK). Isolated SKs occur commonly in the elderly. Sudden onset of multiple SKs (Leser-Trelat sign) suggests an underlying carcinoma of the gastrointestinal tract, most often gastric cancer. It is considered to be a result of a paraneoplastic syndrome associated with the cancer. The best next step in working up a suspected GI malignancy is an abdominal CT scan. Given the high likelihood of malignancy, it would be inappropriate to only reexamine the patient in 2 weeks (A). SKs have a characteristic appearance and typically do not need to be confirmed with a skin biopsy (B). Mohs (D) is a specialized tissue-sparing procedure for treating skin cancer.
It involves tangential excisions of the lesion until margins are negative. Mohs has the advantage in that definitive excision, and closure can be achieved on the same day. Corticosteroids (E) are not used in the management of SKs.

\section{Answer $C$}

Mohs is a specialized tissue-sparing technique of treating skin cancer in which the tumor is removed in a series of thin layers as opposed to one wide excision. The advantage is that it prevents excising excessive normal tissue and allows for immediate confirmation of negative surgical margins intraoperatively. It is best suited for basal cell and squamous cell CA in cosmetically sensitive areas such as the face. Mohs is not generally recommended for melanoma. This is because it is difficult to distinguish the normal skin from melanoma on frozen section (immunohistochemical stains are sometimes needed). Because of this, Mohs is considered by most surgeons to be an unreliable method of resection for melanoma (A, B, D). The treatment of choice for subungual melanoma is digital amputation.

\section{Answer B}

Nodular variant melanomas grow vertically, not horizontally. They are usually a uniformly dark blue or black "berry-like" lesion that is mostly symmetric, elevated, and one color. Impetigo (A) is a superficial bacterial infection oftentimes due to Staphylococcus aureus. It presents first as a flat macule and then a raised pustule that erodes and oozes a dry, honey-crusted serum. A nevus (C), or a mole, is described as a small $(<6 \mathrm{~mm})$ macule with sharp, symmetric borders, and an evenly distributed color. Molluscum contagiosum (D) is caused by the poxvirus and occurs most commonly in children and immunocompromised adults. It is characterized by small, firm, pink, and umbilicated papules. Squamous cell carcinoma (E) is a malignant proliferation of epithelial cells that presents as an ulcerated, nodular mass in sun-exposed areas.

\section{Answer E}

There is a very high likelihood that this patient has an NSTI. After IV fluids, blood cultures, and immediate antibiotics, the next best step is to perform an emergent wide surgical debridement. If the diagnosis of NSTI is uncertain, yet the suspicion is high, surgical exploration is still indicated, as this is the gold standard of both diagnosis and treatment. The incision must be taken down to the fascia and muscle, so both can be inspected. When the diagnosis is in question, plain X-rays (A) are useful as they may demonstrate gas in the soft tissue. CT scan (B) may also be beneficial for the same reason. Duplex scan of leg veins $(C)$ is used to rule out deep venous thrombosis, which can present with leg swelling, but like compartment syndrome (E), it would not cause the laboratory abnormalities described. 


\section{Answer B}

The most common type of lip cancer is squamous cell carcinoma (SCC). Lip cancer occurs much more commonly on the lower lip, as it gets more sun exposure than the upper lip. Occupations that involve long-term sun exposure (e.g., lifeguard, farmer, construction worker, gardener, and field worker) place patients at higher risk for developing skin cancer. SCC is described as an ulcerated, nodular mass without any telangiectasias. Basal cell carcinoma (A) presents as a pearly white nodule with a central ulcerated crater surrounded by dilated vessels (telangiectasias). Lichen planus (C) can be remembered as the "5 Ps": pruritic, planar, polygonal, purple papules. It commonly involves the wrists and elbows and is associated with chronic hepatitis $\mathrm{C}$ infection. One of the manifestations of celiac disease includes dermatitis herpetiformis (D). It presents as pruritic vesicles and bullae that are grouped together (herpetiform). It only occurs in a minority of celiac patients and typically resolves with a gluten-free diet. Melanoma (E) presents as a mole-like growth and follows the ABCDE rule.

\section{Answer B}

Nodular variant melanomas are characterized by the absence of a radial growth phase. They are usually a uniformly dark blue or black "berry-like" lesion that is mostly symmetric, elevated, and one color. They are considered to be the most rapidly growing and aggressive variant of malignant melanoma. Typically, it arises on apparently normal skin (the head, trunk, and neck are the most common locations) vs. preexisting lesion. Ulceration is common, giving a poorer prognosis. Superficial spreading (A) is the most common type of melanoma. It typically has a long horizontal growth phase before the vertical growth phase which confers a better prognosis. Acral lentiginous melanomas (D) are typically found in the subungual (E), sole or palm location, and common in ethnic groups of color. Subungual tend to present late as they are often confused with a subungual hematoma.

\section{Answer $\mathrm{E}$}

Amelanotic and nodular melanomas do not follow the ABCDE rule. As previously mentioned, nodular variant melanomas are characterized by the absence of a radial growth phase. They are usually a uniformly dark blue or black "berry-like" lesion that is mostly symmetric, elevated, and one color. They are considered to be the most rapidly growing and aggressive variant of malignant melanoma. Amelanotic melanomas are notoriously difficult to identify because this variant is deficient in pigment or is unable to produce any pigment at all. For this reason, they typically go unrecognized until the disease advances enough to locally invade the surrounding tissue. Superficial spreading (A) is the most common type of melanoma. It typically has a long horizontal growth phase before the vertical growth phase which confers a better prognosis. Acral lentiginous melanomas (D) are typically found in the subungual, sole or palm location, and common in ethnic groups of color.

\section{Answer A}

A rising WBC and lactate after debridement are highly suggestive of progression of the NSTI. A second-look operation is often required, but in this case would be essential in order to ensure that no additional tissues have become involved since the initial debridement. Amputation (B) may be necessary, but only a second-look operation will indicate whether this is the case. CT scan (D) in the postoperative setting would be difficult to interpret due to postsurgical changes. With severe sepsis, pressors (E) may be necessary, but this would not be the definitive treatment. Furthermore, no hemodynamic parameters (blood pressure, central venous pressure) are provided that would indicate that pressors are needed. Patients with NSTI should always receive broad antibiotic coverage (C) at initial presentation as it is often due to a polymicrobial infection. However, the cornerstone of management is surgical debridement.

\section{Answer B}

Once the diagnosis of melanoma is established by punch or excisional biopsy, the area needs to be re-excised to obtain wider margins, and in select cases, sentinel lymph node biopsy (SLNB) is obtained. The extent of margins and need for SLNB are determined by tumor thickness. Patients with tumor thickness $<1 \mathrm{~mm}$ (considered a thin melanoma) require an excision margin of only $1 \mathrm{~cm}$. For melanomas that are thin, SLNB is generally not performed. Interferon alpha (C), GM-CSF (D), and dacarbazine (E) are all adjuvant therapy options for patients with melanoma. There has been no concrete evidence that adjuvant therapy prolongs survival in melanoma. However, there is some evidence to suggest that there is an improved relapse-free survival and overall survival with high-dose interferon alpha.

\section{Answer A}

Actinic keratosis presents as a rough, scaly patch of the skin that can vary in color (pink, red, brown). It is the most common precancerous skin lesion. It can progress to squamous cell carcinoma. Seborrheic dermatitis (cradle cap) (B) is a self-limited condition that commonly affects infants and presents as a yellow, greasy plaque on the scalp. Seborrheic keratosis (C) is a common tumor in the elderly and presents as raised, discolored plaques that appear coinlike, waxy, and with a "stuck-on" appearance. Both of these conditions are benign and not considered to be precancerous. 
Compound nevi (D) are brown-black, well-circumscribed lesions that are $<1 \mathrm{~cm}$ in diameter. They may be elevated and are frequently hairy, arising from the epidermal-dermal interface and from within the dermis. Malignant transformation is rare. Keratoacanthoma $(\mathrm{E})$ is a low-grade subtype of squamous cell carcinoma that can grow rapidly and become large in size. Most will spontaneously get better within a year, but removal with surgery is still recommended.

\section{Answer D}

Melanocytes are found in equal numbers in most people (black or white). However, individuals with darker skin have melanocytes that produce more melanin, a protein which makes skin darker and helps protect from skin cancer by absorbing UV-B (B for bad) radiation. As such, in darkskinned patients, melanomas are more likely to occur in areas that have less pigmentation such as the palms, soles, and mucous membranes. 


\section{Surgical Complications}

\section{Areg Grigorian, Paul N. Frank, Christy Anthony, and Christian de Virgilio}

\section{Questions}

1. A 55-year-old woman arrives to the emergency department with a 3-h history of severe epigastric pain that has been progressing in severity. She describes it as sharp, diffuse, and constant. Her past medical history is significant for chronic atrial fibrillation for which she takes warfarin. Her temperature is $100.6^{\circ} \mathrm{F}$, blood pressure is $102 / 66 \mathrm{mmHg}$, and pulse is $98 / \mathrm{min}$ and irregular. Physical examination reveals a diffusely tender and rigid abdomen, with guarding and rebound. An upright abdominal $\mathrm{x}$-ray demonstrates free air under the right hemidiaphragm. Her laboratory exam findings include a hemoglobin $10.2 \mathrm{~g} / \mathrm{dl}$ (normal 12-15 g/dl), platelets 110,000 (150,000-400,000), INR 2.5, and PTT $18 \mathrm{~s}$ (18-28 s). Which of the following is the best way to manage the INR in this patient?
(A) Hold warfarin and allow INR to autocorrect
(B) Oral vitamin $\mathrm{K}$
(C) Fresh frozen plasma
(D) Intravenous vitamin $\mathrm{K}$
(E) Cryoprecipitate

2. What is the most common CXR finding in a patient with pulmonary embolism (PE)?
(A) Consolidation of one lobe
(B) Fluffy bilateral infiltrates
(C) Normal
(D) Hampton's hump (a wedge-shaped, pleural-based consolidation)
(E) Westermark's sign (a focus of oligemia leading to collapse of pulmonary vessel)

3. What is the most common ECG finding in a patient with PE?
(A) Right ventricular strain
(B) Right-axis deviation
(C) $\mathrm{S}_{1} \mathrm{Q}_{3} \mathrm{~T}_{3}$
(D) Sinus tachycardia
(E) Right bundle branch block

4. What is the most common initial acid/base abnormality seen in patient with a PE?
(A) Respiratory alkalosis
(B) Respiratory acidosis
(C) Metabolic alkalosis
(D) Metabolic acidosis
(E) Combined respiratory alkalosis and metabolic acidosis

5. A 61-year-old obese female with a past medical history of diabetes undergoes laparoscopic cholecystectomy for acute cholecystitis. The operation is technically difficult and is converted to an open cholecystectomy. Twelve hours later, the patient complains of severe pain in the wound. She has a temperature of $102.8^{\circ} \mathrm{F}$, heart rate of $120 / \mathrm{min}$, and a blood pressure of $110 / 70 \mathrm{mmHg}$. She appears ill. There is grayish foul-smelling drainage coming from the wound, which appears erythematous, swollen, indurated, and tender to touch. What is the next step in the management?

(A) Reassure patient that wound infections do not occur so soon after surgery

(B) Open a few of the wound staples to allow drainage

(C) Broad-spectrum antibiotics

(D) Return to OR for reclosure of the fascia

(E) Broad-spectrum antibiotics and return to OR for aggressive wound debridement

6. A 29-year-old female who is 8 months pregnant presents with symptoms and signs of acute cholecystitis and undergoes laparoscopic cholecystectomy uneventfully. However, she returns to the ED on postoperative day 4 with acute onset dyspnea and pleuritic chest pain. A pulmonary embolism is subsequently diagnosed. What is the most likely source of the thromboembolism?
(A) The right common iliac vein
(B) The left common iliac vein
(C) The right popliteal vein
(D) The right axillary vein
(E) The left femoral vein

7. A 50-year-old female undergoes right hepatic lobectomy for metastatic colon cancer. The operation took $6 \mathrm{~h}$ and was associated with significant bleeding. As a result, she was markedly hypotensive throughout the operation and received multiple units of blood products. She was admitted to the surgical ICU for further management. She received one dose of prophylactic antibiotics preoperatively, but is otherwise not receiving any medications. On postoperative day 1 , her urine output is only $10 \mathrm{cc} / \mathrm{h}$ for $5 \mathrm{~h}$. Her serum creatinine has risen from 1.2 to $2.0 \mathrm{mg} / \mathrm{dl}$. Her blood pressure is $140 / 80 \mathrm{mmHg}$, and heart rate is $100 / \mathrm{min}$. Lungs are clear to auscultation. Urine $\mathrm{Na}$ is $44 \mathrm{mEq} / \mathrm{L}$, and FENa is $3.1 \%$. Which of the following is the most likely etiology?
(A) Hypovolemia
(B) Acute interstitial nephritis
(C) Acute tubular necrosis
(D) Cardiogenic shock
(E) Obstructed urinary catheter

8. A 64-year-old female was recently discharged following a morbid obesity surgery (gastric sleeve resection). 
Her postoperative course was complicated by a DVT in her left leg, for which she received intravenous heparin and discharged with oral warfarin. She now presents with an area of discolored, purplish skin on her right thigh that began the prior day and is extremely painful. On physical exam, she is afebrile with a normal blood pressure and heart rate. There is a $6 \times 6 \mathrm{~cm}$ area of purplish black skin over her right anterior thigh, and another smaller area on her right calf. On laboratory exam, her WBC count and serum glucose are normal. What is the most likely cause?
(A) Vitamin $\mathrm{K}$ deficiency
(B) Protein C deficiency
(C) Heparin
(D) Thrombocytopenia
(E) Unrecognized hemophilia

9. A 40-year-old male is hospitalized after a hip replacement. On postoperative day 3 , the astute medical student notices he has a new left-sided facial droop. Cardiac examination demonstrates a regular rate and rhythm without murmurs. Further exam reveals left arm and leg weakness and numbness. In addition, he has a swollen right calf that is $3 \mathrm{~cm}$ larger in diameter than the left calf when measured $10 \mathrm{~cm}$ below the tibial tubercle. Venous duplex ultrasound shows a noncompressible right femoral vein. What test is most likely to explain the etiology of the neurologic findings?
(A) ECG
(B) CT of the chest
(C) Factor V Leiden testing
(D) Echocardiogram with bubble study
(E) Duplex ultrasound of the carotid artery

10. Which of the following findings on urinalysis would most strongly support the diagnosis of acute tubular necrosis?
(A) Muddy brown casts
(B) Urine osmolarity $>500$
(C) Bland urine sediment
(D) Red cell casts
(E) White cell casts

11. A 65-year-old homeless man with poorly controlled diabetes presents to urgent care with severe pain and swelling in his left leg. Vital signs include a temperature of $102{ }^{\circ} \mathrm{F}$, blood pressure of $132 / 78 \mathrm{mmHg}$, and heart rate of $102 / \mathrm{min}$. On physical exam, he appears to be tachypneic. His left leg appears tense, and the skin is warm and red over his thigh and is tender to palpation. There is an area of the skin on the thigh that has a violaceous color with blistering. Laboratory testing demonstrates a WBC $22 \times 10^{3} / \mu \mathrm{L}$ (normal $4.1-10.9 \times 10^{3} / \mu \mathrm{L}$ ) with neutrophilic predominance and a serum $\mathrm{Na}$ of $128 \mathrm{mEq} / \mathrm{L}$
(137-145 $\mathrm{mEq} / \mathrm{L})$. What is the best next step in treatment?

(A) Immediate IV heparin

(B) Venous duplex scan of left leg

(C) CT pulmonary angiogram

(D) Blood cultures, broad-spectrum antibiotics, and urgent surgical debridement

(E) Blood cultures and broad-spectrum antibiotics

12. A 78-year-old male is in the recovery room after an open inguinal hernia repair. His blood pressure is noted to be $70 / 55 \mathrm{mmHg}$, and pulse is $118 \mathrm{bpm}$. He is breathing normally. The patient has a history of hypertension for which he takes a diuretic. The operation itself was uneventful except that the nurses had difficult inserting the urinary catheter. Given the hypotension, the patient is transferred to the ICU for close monitoring. The following hemodynamic parameters are obtained: cardiac output of $10.2 \mathrm{~L} /$ min (normal $5 \mathrm{~L} / \mathrm{min}$ ) and systemic vascular resistance of $450 \mathrm{dynes} / \mathrm{sec} / \mathrm{cm}^{5}$ (normal 700-1,600 dynes/sec/ $\left.\mathrm{cm}^{5}\right)$. What is the next step in treatment?
(A) Phenylephrine
(B) Dopamine
(C) Blood cultures and broad-spectrum antibiotics
(D) IV bolus of lactated Ringer's
(E) Norepinephrine

13. A 25-year-old woman is referred to an ENT surgeon with complaints of recurrent nosebleeds for the past month. She also reports that she has been easily bruising with minor trauma and her last menstrual period required double the change of tampons she typically uses. She does not report any blood in stool or urine. Her physical exam is significant for mild gingival bleeding and scattered bruises on her arms and legs. Her laboratory exam is significant for an isolated thrombocytopenia of 13,000 (normal 150,000-400,000). Bone marrow biopsy is normal other than an increase in megakaryocytes. What is the best initial management for this condition?
(A) Platelet transfusion
(B) Corticosteroids
(C) Splenectomy
(D) Plasmapheresis
(E) Intravenous immunoglobulin (IVIG)

14. A 60-year-old female returns to the ED for right leg swelling 1 week after undergoing a right hemicolectomy for cecal adenocarcinoma. Duplex scan confirms a DVT and the patient is started on IV heparin. The patient's hospital course is further complicated by UTI and pneumonia. On hospital day 7 , the morning CBC shows a platelet count of 55,000, down from a baseline of 140,000 . What is the next immediate step? 

(A) Stop heparin
(B) Stop heparin and start a direct thrombin inhibitor
(C) Stop heparin and switch to low molecular weight heparin
(D) Transfuse 2 units of platelets
(E) Start corticosteroids

15. A 68-year-old female presents to the ED 1 week after total hip replacement. She became suddenly short of breath $2 \mathrm{~h}$ ago. Her vitals include a blood pressure of $100 / 60 \mathrm{mmHg}$, heart rate of $120 / \mathrm{min}$, and respiratory rate of 30/min. On physical examination, lung sounds are clear. Chest $\mathrm{x}$-ray is normal. Arterial blood gas shows a $\mathrm{pH}$ of $7.53, \mathrm{PaCO}_{2} 28, \mathrm{PaO}_{2} 70$, and $\mathrm{HCO}_{3} 25$. Oxygen is given by nasal cannula. Which of the following is the next best step in the management?
(A) Spiral CT angiogram of the chest
(B) Intravenous heparin
(C) Intravenous thrombolytic infusion
(D) Venous duplex scan of both legs
(E) Echocardiogram

16. A 45-year-old patient undergoes a hernia repair. He is a smoker. On postoperative day 2, his wound appears to be healing well, and he is discharged home. What type of operative wound is this considered to be?
(A) Clean
(B) Clean contaminated
(C) Contaminated
(D) Dirty infected
(E) Elective

17. A 51-year-old male is brought to the ED by paramedics following a high-speed MVC. In the ED, his blood pressure is $120 / 70 \mathrm{mmHg}$, and heart rate is $100 / \mathrm{min}$. Hemoglobin/hematocrit is $12 \mathrm{~g} / \mathrm{dL}(13.2-16.2 \mathrm{~g} / \mathrm{dL})$ and $36 \%(40-52 \%)$, respectively. A CT scan shows a ruptured spleen, and he is taken urgently to the operating room. During surgery, the patient is hemodynamically stable and undergoes a splenectomy. No other injuries are found, and he does not require blood transfusion. Four hours postoperatively, the patient's blood pressure drops to $80 / 50 \mathrm{mmHg}$, heart rate is $120 / \mathrm{min}$, and urine output, which was $50 \mathrm{cc} / \mathrm{h}$ for the first $2 \mathrm{~h}$ after surgery, is $10 \mathrm{cc} / \mathrm{h}$ for the past $2 \mathrm{~h}$. The patient is awake and only complains of thirst. He appears pale. Breath sounds are clear. Despite two liters of IV fluids, the blood pressure remains $80 / 50 \mathrm{mmHg}$. Repeat hemoglobin/hematocrit is $10 \mathrm{~g} / \mathrm{dL}$ and $30 \%$. What is the next step in the management?
(A) CT scan
(B) Diagnostic peritoneal lavage
(C) 12-lead ECG
(D) Chest X-ray
(E) Return to operating room 


\section{Answers}

\section{Answer C}

The ideal method for reversing an elevated INR in a patient receiving warfarin depends on the urgency with which reversal is needed. The patient presented has peritonitis and free air under the diaphragm. As such, urgent surgery is required, and therefore urgent reversal of INR is necessitated. Of the choices provided, fresh frozen plasma (FFP) would provide the most immediate reversal. Holding warfarin (A) is not acceptable as it would take 3-4 days for reversal. Oral vitamin K (B) takes about $24 \mathrm{~h}$, whereas IV vitamin K (D) takes about $8-12 \mathrm{~h}$. Such strategies would be acceptable if surgery were not urgent and would have the benefit of avoiding transfusion of a blood product (FFP). Cryoprecipitate (E) has high levels of fibrinogen and von Willebrand factor but is not effective for warfarin reversal. Prothrombin complex concentrate is emerging as an alternative to FFP for rapid warfarin reversal. It more effectively reverses warfarin as compared to FFP (particularly in patients who are massively bleeding and have profoundly elevated INR), though it is associated with an increased risk of thrombotic events.

\section{Answer C}

Virchow's triad (hypercoagulability, immobility, endothelial injury) is a common risk factor for PE. Patients can present with dyspnea, pleuritic chest pain, cough, tachycardia, and/ or hemodynamic instability. Most will have a normal appearing chest $\mathrm{x}$-ray. If present, the most common abnormality is a platelike atelectasis with decreased lung volume. Hampton's hump (D) is seen in $20 \%$ of patients with PE and is characterized by a wedge-shaped, pleural-based consolidation frequently seen laterally. Westermark's sign (focal/regional pulmonary oligemia distal to embolus) (E) is even more rare and only seen in cases of saddle embolus. Consolidation of an entire lobe (A) is more consistent with lobar pneumonia. Fluffy bilateral infiltrates (B) seen on plain films are suggestive of pulmonary edema.

\section{Answer D}

The most common ECG finding in patients with PE is sinus tachycardia. $\mathrm{S}_{1} \mathrm{Q}_{3} \mathrm{~T}_{3}(\mathrm{C})$ refers to a deep $\mathrm{S}$ wave in lead $\mathrm{I}, \mathrm{Q}$ wave in III, and inverted T wave in III, and although it is considered a "classic" finding for PE, it is neither sensitive nor specific and found in only $20 \%$ of patients. When pulmonary arterial pressures get high, ECG findings may demonstrate right ventricular strain (A). The remaining answer choices can all occur in patients with PE but appear infrequently (B, C, E).

\section{Answer A}

The most common acid/base abnormality seen initially in patients with PE is uncompensated respiratory alkalosis. Patients with PE can have hypoxia, and the physiologic response to this is hyperventilation which results in a primary decrease in $\mathrm{PCO}_{2}$ (hypocapnia). Acute respiratory alkalosis causes light-headedness, confusion, peripheral paresthesias, cramps, and syncope. Eventually patients will have a compensated respiratory alkalosis as the body's excess $\mathrm{HCO}_{3}{ }^{-}$is buffered by extracellular hydrogen ion. Combined respiratory alkalosis and metabolic acidosis is seen initially in patients that have ingested a large amount of aspirin (E).

\section{Answer $E$}

The vast majority of fevers within the first $24 \mathrm{~h}$ of surgery are noninfectious in origin and are due to release of cytokines (not atelectasis as commonly stated \{but before you challenge your chief residents, look it up for yourself!\}). Postoperative wound infections typically do not occur until around the seventh postoperative day on average. There are rare exceptions. Group A beta-hemolytic Streptococcus and Clostridium are known to rarely cause devastating early (sometimes within hours) postoperative wound infections. For this reason, when a patient spikes a fever, always perform a directed physical exam, including looking at the wound. Clues to a wound infection include a warm, erythematous, painful wound with dishwater (grayish) foul-smelling discharge. Management consists of immediate broad-spectrum antibiotics (including high-dose penicillin to cover Clostridium) and a prompt return to the OR for aggressive wound debridement. Reassurance (A), bedside drainage (B), and antibiotics alone (C) would not be appropriate. Reclosure of the fascia (D) would be appropriate for fascial dehiscence. However, fascial dehiscence presents with a large volume of salmon-colored fluid draining from wound, not with fever and evidence of wound infection.

\section{Answer B}

DVTs occur more commonly in the left leg than the right due to the fact that the right common iliac artery often compresses the left common iliac vein (this condition is termed May-Thurner syndrome). The risk of DVT is further increased in pregnancy due to the gravid uterus causing further compression. The other sites listed are less common locations of DVT (A, C-E). Pregnant women cannot be treated with warfarin due to its teratogenicity.

\section{Answer C}

The patient is oliguric and has evidence of acute kidney injury (AKI). The high urine $\mathrm{Na}(>40 \mathrm{mEq} / \mathrm{L})$ and FENa $>1 \%$ indicate an intrinsic (renal) etiology of AKI. The most common cause of renal AKI is acute tubular necrosis (ATN). Hypovolemia (A) causes prerenal azotemia. Prolonged periods of hypovolemia and hypotension lead to poor renal perfusion that directly damage the kidneys and lead to acute tubular necrosis (ATN). Acute interstitial nephritis (B) also causes intrarenal AKI. However, it is less common than ATN 
and is an immune-mediated response to certain medications (e.g., penicillin, cephalosporins, sulfa drugs, NSAIDs). Classic findings include fever, rash, arthralgia, and urinary eosinophilia. Cardiogenic shock (D) can lead to prerenal AKI due to decreased renal perfusion. Prolonged urinary obstruction due to bilateral ureteral obstruction can lead to postrenal AKI. Urine findings are variable. An obstructed Foley (E) is a potential cause of oliguria, but is unlikely to cause AKI and certainly not so soon after surgery.

\section{Answer B}

The history and exam are most consistent with warfarininduced skin necrosis. Warfarin inhibits the carboxylation of the vitamin K-dependent clotting factors: II, VII, IX, X, protein $\mathrm{C}$, and protein $\mathrm{S}$. This can acutely lead to the relative deficiency of protein $\mathrm{C}$, owing to its short half-life, and thus can result in an initial hypercoagulable state and subsequent thrombosis in the vasculature supplying the skin. Warfarininduced skin necrosis is more common in patients who have a preexisting protein $\mathrm{C}$ deficiency. Vitamin $\mathrm{K}$ deficiency is seen with severe nutritional depletion and intestinal malabsorption and manifests with bruising and hemorrhage (A). Heparin can cause skin necrosis as well, but this is seen locally at the site of injection (patient however received IV heparin) and in a much smaller distribution (C). Thrombocytopenia results in petechiae, not skin necrosis (D). Patients with hemophilia may have a history of deep tissue bleeding into muscles and joints (hemarthrosis) and oftentimes have excessive bleeding after surgical procedures (E), but not skin necrosis.

\section{Answer D}

The patient has symptoms and signs of a postoperative stroke. Most postoperative strokes are ischemic in nature (not hemorrhagic), and most ischemic strokes are embolic, arising from either the heart (in the setting of atrial fibrillation) or from a plaque at the carotid bifurcation in the neck. This patient, however, has a DVT. The combination of an acute DVT and a stroke suggests a paradoxical embolism, wherein a clot from the venous system enters the systemic (as opposed to pulmonary) circulation. The most likely explanation is an intracardiac shunt such as patent foramen ovale (PFO) or atrial septal defect (ASD). Such an anomaly would best be demonstrated with an echocardiogram with a bubble study. ECG (A) might be helpful if atrial fibrillation was suspected; however, the patient has a regular rate and rhythm. In the setting described above, a CT of the head would be the first study indicated. Head CT would confirm whether the patient did have a stroke, and whether the stroke was ischemic or hemorrhagic (but this was not an option), but CT would not be helpful for determining the source of an embolic stroke. CT of the chest (B) would be helpful if PE were suspected. Factor V Leiden testing is not routinely recommended following a first-time DVT. Duplex ultrasound of the carotid arteries (E) may identify a plaque, but he is very young to have a carotid stenosis, and the concomitant DVT should raise a higher suspicion for paradoxical embolism.

\section{Answer A}

In acute tubular necrosis, the renal tubular epithelial cells die and slough off into the urine. These appear as muddy brown casts. Urine osmolarity $>500$ (B) and bland urine sediment (C) are both consistent with a prerenal AKI state. Red cell casts (D) are suggestive of injury to the glomerulus (e.g., glomerulonephritis). White cell casts (E) are suggestive of tubulointerstitial disease or acute pyelonephritis but may also be observed with many glomerular disorders.

\section{Answer D}

The patient is homeless, which predisposes him to unsanitary conditions. Poorly controlled diabetes itself is an immunosuppressed state. Given this information about the patient, the presence of leukocytosis with neutrophilic predominance, and his physical exam findings (e.g., painful, erythematous, swollen leg with bullae and violaceous skin), this patient likely has necrotizing fasciitis. Management consists of blood cultures, broad-spectrum antibiotics, and urgent surgical debridement. Antibiotics and cultures alone would not be appropriate (E). Choices A-C are all appropriate considerations for patients presenting with a $\mathrm{PE}$ secondary to DVT.

\section{Answer D}

The patient is in shock. Given the high cardiac output, and low systemic vascular resistance, septic and anaphylactic shock are the most likely. However, since the patient has not had any medications or unusual exposures and is breathing normally, it is most likely septic shock. Patients with difficult urinary catheterizations may have subsequent bacteremia which can result in septic shock. The first step in management of septic shock is aggressive IV fluid resuscitation with either normal saline (NS) or lactated Ringer's (LR). Norepinephrine (E) is considered as the first-line vasopressor for septic shock. Additionally, vasopressin can be used in combination with norepinephrine. Epinephrine is also used for septic shock, but after the above two. Dopamine (B) was initially believed to increase renal perfusion in patients with shock, but studies have failed to consistently demonstrate this. It is not typically recommended for patients with septic shock (except for the rare patient with associated bradycardia). Phenylephrine (A) is not recommended for septic shock except in highly selected patients. All patients with septic shock should also receive blood cultures before starting broad-spectrum antibiotics (C).

\section{Answer B}

In a young patient presenting with recurrent epistaxis, isolated thrombocytopenia, and bleeding symptoms, an isolated 
acquired thrombocytopenia should be considered. Immune thrombocytopenic purpura (ITP) is an autoimmune disease characterized by autoantibodies against platelets and thus is considered a consumptive process. In a patient with a platelet count $<30,000$ and bleeding symptoms, the recommended initial management is corticosteroids (B). If this does not control the symptoms, the next line of therapy includes IVIG (E), dapsone, or danazol. Splenectomy is considered a last resort after medical therapy fails $(\mathrm{C})$. Platelet transfusion should never be administered to a patient with ITP because it is considered a consumptive process (A). Plasmapheresis is not one of the recommended treatments for ITP (D).

\section{Answer B}

The findings here are consistent with heparin-induced thrombocytopenia (HIT), an immune reaction to heparinplatelet complexes. Despite the decrease in platelet count, this is a hypercoagulable state. Heparin should be discontinued immediately, and the patient should be started on a direct thrombin inhibitor (e.g., argatroban). Low molecular weight heparin (C) also has a risk of HIT. Platelet transfusion (D) is not indicated with a platelet count of 55,000. Corticosteroids (E) would not be appropriate for the management of HIT.

\section{Answer B}

The patient presented has a high likelihood of PE, based on Wells criteria. The patient is in the postoperative setting, the CXR is normal, and the patient is tachycardic and slightly hypotensive, suggesting possible right heart strain from a massive PE. As such, the first step is to immediately start IV heparin, even before the diagnosis is confirmed with spiral CT (A). In patients with a low likelihood of PE, a D-dimer assay should be ordered first. Since D-dimer levels are often elevated after surgery, it has very poor specificity in the postoperative setting. That being said, D-dimer has a high negative predictive value. So even though most postoperative patients will have an elevated D-dimer, D-dimer level $<500 \mathrm{ng} / \mathrm{mL}$ effectively can rule out PE in low-risk patients. IV thrombolytic infusion (C) would not be an appropriate management because the patient just had major surgery. Venous duplex scan of both legs (D) could subsequently be done to evaluate for DVT as the source for PE. Echocardiogram (E) with a bubble study would be appropriate if the patient presented with a combined stroke and acute DVT, suggestive of a paradoxical embolism.

\section{Answer A}

There are four types of operative wounds. These are used to predict the likelihood of a postoperative wound infection and to guide the use of preoperative antibiotics. Clean wounds (class I) are those that do not involve entering an organ or cavity that is known to harbor bacteria (such as the alimentary, genitourinary, or reproductive tracts). Examples would be skin, eye, brain, or elective orthopedic surgery (and also hernia repair). Clean-contaminated (class II) wounds (B) are those in which an aseptically made wound enters the alimentary, respiratory, or genitourinary tracts (e.g., elective bowel resection, caesarean section). Contaminated (class III) wounds (C) occur secondary to trauma, breaks in sterile technique, or gross spillage from the gastrointestinal tract (e.g., GSW to the bowel). Dirty-infected (class IV) wounds (D) are those involving a preoperative infection (drainage of an abscess) or perforated viscera (e.g., abscess). Elective wound $(\mathrm{E})$ is not a recognized type of wound. Patients that have optimized oxygen delivery to tissue are most likely to have normal wound healing. Factors known to increase the risk of wound infections include wound ischemia, diabetes, low albumin, steroids, poor arterial flow (e.g., peripheral arterial disease), smoking, and hypothermia.

\section{Answer E}

The patient is in shock. The most likely etiology of the shock in this setting is hypovolemic shock, most likely from bleeding (in this case most likely the ligation of the splenic artery has come off). Massive postoperative bleeding after surgery is rare and can be difficult to recognize. It can present as oliguria, and differentiating bleeding from other causes of hypotension and oliguria can be difficult. Lab values such as hemoglobin and hematocrit are not useful in detecting acute hemorrhage in the postoperative setting. It generally takes 8-12 h for interstitial fluid to redistribute into the vascular space, and blood concentration will initially appear unchanged. This patient's hemoglobin/hematocrit has shown a significant drop, and in combination with his thirst, pale skin, mechanism of injury, and hemodynamic instability, he is most likely suffering from internal bleeding and will need to return to the OR. CT scan would be inappropriate for a patient with hemodynamic instability (A). An ECG (C) or a CXR (D) (the breath sounds are clear) are unlikely to be of value. Failure to recognize bleeding has fatal consequences. An alternative to immediate return to the OR would be to perform a bedside ultrasound (to look for fluid), if available. 


\section{Trauma}

\section{Areg Grigorian, Paul N. Frank, Christian de Virgilio, and Dennis Y. Kim}

\section{Questions}

1. A 25-year-old man arrives to the ED following a MVC with multiple abrasions on his abdomen. His blood pressure is $90 / 60 \mathrm{mmHg}$, and his pulse is $120 / \mathrm{min}$. After a primary survey, a FAST exam is performed. Which of the following is a FAST exam poor at detecting?
(A) Pericardial effusion
(B) Single pneumothorax
(C) Free peritoneal fluid in the hepatorenal space (Morrison's pouch)
(D) Retroperitoneal fluid
(E) Free peritoneal fluid in the perisplenic space

2. A 23-year-old male is rushed to the ED by paramedics after sustaining a gunshot wound to the lateral neck at the level of the thyroid cartilage. The patient is hemodynamically stable and is able to speak. Physical exam shows no signs of hematoma, pulsatile bleeding, thrill, or bruit. Which of the following is the next step in management?
(A) Surgical exploration
(B) Wound closure
(C) CT angiography
(D) Intubation
(E) Formal angiography

3. A 41-year-old patient presents to the emergency department following a stab wound to the chest, just above the left nipple line. On initial exam, his blood pressure is $94 / 70 \mathrm{mmHg}$, and respiratory rate is $16 / \mathrm{min}$. He has distended neck veins, and his heart sounds are muffled. A FAST exam demonstrates fluid in the pericardial sac. What is considered the first sign of this condition?
(A) Electrical alternans
(B) Impaired diastolic filling
(C) "Water-bottle" shape on chest radiograph
(D) Hypotension
(E) Distended neck veins

4. A pregnant woman in her second trimester arrives to the ED after a minor MVC. She has no injuries or complaints but is worried that her pregnancy is in danger. She has a nonstress test that shows two accelerations of fetal heart rate, each at least 15 beats per minute above baseline and lasting at least $15 \mathrm{~s}$. She has no contractions, vaginal bleeding, or abdominal pain. A FAST exam is negative. What is the next best step in management?
(A) Monitor the patient overnight
(B) Biophysical profile
(C) Discharge and follow-up in 2 weeks
(D) CT of the abdomen
(E) MRI of the abdomen

5. A 40-year-old policeman is brought to the ED having suffered burns after helping to rescue a woman from a burning warehouse. His temperature is $99.8{ }^{\circ} \mathrm{F}$, blood pressure is $100 / 70 \mathrm{mmHg}$, pulse is $95 / \mathrm{min}$, and respiratory rate is $24 / \mathrm{min}$. On physical examination, he has $40 \%$ total body surface area deep partial and full-thickness burns to his face, arms, and back as well as a circumferential burn of his neck. He has singed nasal hairs, and there is carbonaceous sputum coming out of his mouth. His lungs are clear to auscultation bilaterally. ECG demonstrates premature ventricular contractions. What is the most appropriate next step in management?
(A) Broad-spectrum antibiotics
(B) Endotracheal intubation
(C) IV fluid resuscitation
(D) Cardiac enzymes and serial ECG
(E) Bronchoscopy

6. A 40-year-old man falls down approximately three stories in an attempt to commit suicide. EMS arrives on scene within $5 \mathrm{~min}$, and he is rushed to the ED but loses vitals in the field and is dead on arrival (DOA). What is the most likely cause of death?

(A) Thoracic aortic transection

(B) Tension pneumothorax

(C) Abdominal aortic transection

(D) Ruptured spleen

(E) Pulmonary artery transection secondary to a jagged rib edge

7. A 25-year-old football player presents to the ED after sustaining a devastating tackle and hyperextension of his right knee. The knee appears to be posteriorly dislocated and the leg is swollen. Pedal pulses on the right appear to be diminished but present, whereas they are normal on the left. The remainder of his exam does not reveal any obvious signs of bleeding. What is the appropriate next step in management?

(A) Fasciotomy of all four compartments of the lower leg

(B) CT angiography

(C) Immediate heparinization

(D) Plain film of the knee, followed by reduction of the dislocation

(E) MRI of the knee

8. A 30-year-old male arrived via paramedics after getting struck in the abdomen by a golf cart while vacationing 
with his family. He had no head trauma and only complained of mild abdominal pain. His vitals were normal and stable. A CT scan revealed no abnormal findings, and he was discharged on the same day. Three days later, he comes back to the ED complaining of fevers, nausea, poor appetite, and abdominal pain. A repeat CT scan shows a laceration at the neck of the pancreas with disruption of the pancreatic duct. What is the best next step in management?

(A) Order serum amylase

(B) Endoscopic retrograde cholangiography (ERCP)

(C) CT-guided drainage

(D) Magnetic resonance cholangiopancreatography (MRCP)

(E) Exploratory laparotomy

9. A 40-year-old man is in a head-on MVC with a drunk driver on the freeway and is brought to the ED. He has a dark bruise from his seat belt across the left side of his neck. On physical examination, he is neurologically intact. However, his left eyelid is drooping, and his left pupil is constricted as compared to his right. CT scan with contrast demonstrates dissection of the left internal carotid that extends into the base of the skull. CT of the head and abdomen are negative. Which of the following would be the most appropriate management?
(A) Left neck exploration
(B) Intravenous heparin administration
(C) Carotid stenting
(D) Thrombolytic therapy
(E) Observation

10. A 22-year-old male arrives to the ED by paramedics with a gunshot wound in the RUQ of his abdomen. He is anxious and complains of pain near his wound. His temperature is $99.1{ }^{\circ} \mathrm{F}$, blood pressure is $114 / 78 \mathrm{mmHg}$, and pulse is $90 / \mathrm{min}$. His abdomen is soft, and he has no rebound or guarding. A portable chest $\mathrm{x}$-ray is normal, and nasogastric tube (NGT) demonstrates clear fluid with no blood. His rectal examination shows no blood. What is the most appropriate next step in management?
(A) Serial physical examination
(B) Exploratory laparotomy
(C) CT scan of the abdomen
(D) Diagnostic peritoneal lavage
(E) FAST exam

11. A 62-year-old man with atrial fibrillation presents to the ED with a painful right lower leg. He has refused warfarin in the past. His physical exam is significant for an irregularly irregular heart rate and a painful right leg that is cool to touch with absent distal pulses. Pulses in the left foot are normal. He has significant motor weakness and sensory deficit in the right foot. Duplex scan reveals an occlusion of the right popliteal artery. He receives heparin and undergoes open surgical embolectomy. Following the procedure, his motor and sensory deficit dramatically improves. The next day, he experiences intense pain in the right calf. His right calf is swollen and tense, and the pain is worsened with passive dorsi and plantar flexion of his right foot. He has palpable distal pulses. What is the most likely underlying etiology for his acute condition?
(A) Interstitial edema
(B) Recurrent embolization
(C) Deep vein thrombosis (DVT)
(D) Atherosclerotic plaque
(E) Lymphedema

12. Burn patients are at risk for multiple infections. What is the most common organism to cause infection in burn patients?
(A) Staphylococcus aureus
(B) Streptococcus pyogenes
(C) Streptococcus agalactiae
(D) Pseudomonas aeruginosa
(E) Candida albicans

13. A 10-year-old boy presents to the ED with severe abdominal pain after falling over his bicycle handles while attempting a trick and sustaining blunt injury to the abdomen. A CT scan shows oral contrast extravasation into the retroperitoneum that is coming from the posterior aspect of the duodenum. Which of the following is the best management recommendation?
(A) Laparoscopy
(B) Exploratory laparotomy
(C) Observation
(D) Upper endoscopy to confirm injury
(E) CT-guided drainage

14. A 40-year-old alcoholic presents to the ED with a markedly swollen right forearm that is diffusely tender. $\mathrm{He}$ states that following an alcohol and heroin binge, he fell asleep on his arm for $12 \mathrm{~h}$. He woke up to find his hand completely numb and unable to move it. On physical exam, he has normal brachial and radial pulses. His heart has a regular rate and rhythm. He is unable to extend his wrist when the hand is palm down. ECG reveals peaked T waves, and CPKs are 20,000 IU/L (normal 60-400 IU/L). What is the next step in management?

(A) Propranolol

(B) Insulin + dextrose

(C) Calcium gluconate 


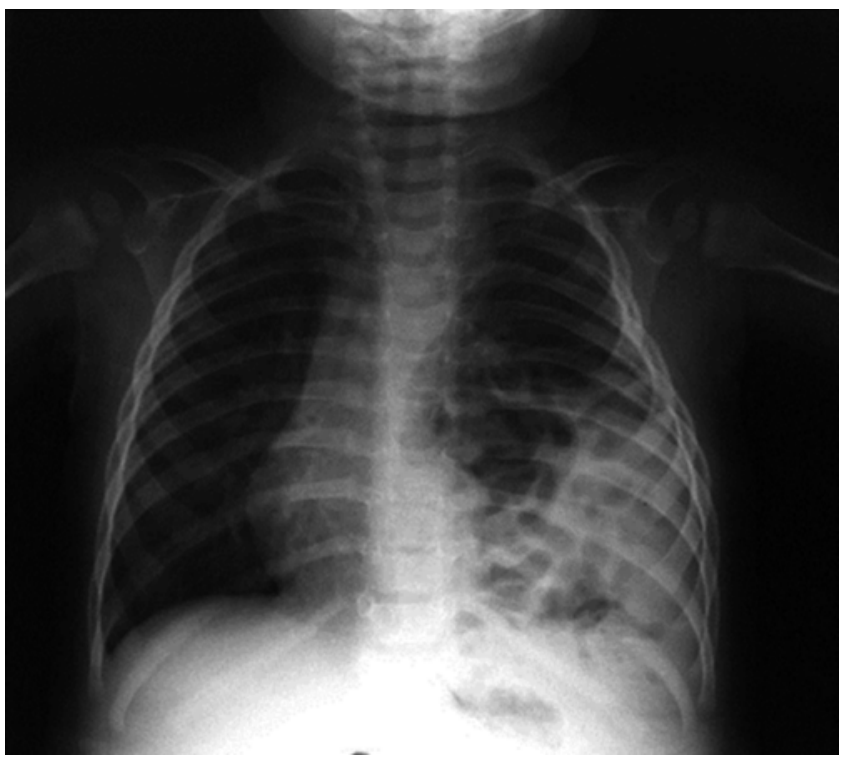

Fig. A.1 Chest $\mathrm{x}$-ray (With kind permission from Springer Science+Business Media: Pediatr Surg Int, An unusual late presentation of a congenital diaphragmatic hernia, 21, 2005, p 1022, Yamamoto H. and Parikh DH., Fig. 2)

(D) Kayexalate (sodium polystyrene)

(E) Furosemide

15. A 5-year-old girl arrives to the ED with complaints of nausea, vomiting, and abdominal pain for the past day. She has no significant past medical history, but her mother reports that she was involved in a MVC about a month ago. She was restrained in a car seat and had blunt trauma to her abdomen. She had no complaints at the time. Her vital signs were normal, and she was subsequently discharged a few hours later. Her blood pressure is currently $112 / 82 \mathrm{mmHg}$, pulse is $90 / \mathrm{min}$, and respiratory rate is $28 / \mathrm{min}$. Her chest $\mathrm{x}$-ray is shown above (Fig. A.1). What is the most likely diagnosis?
(A) Gastroenteritis
(B) Diaphragmatic hernia
(C) Delayed splenic rupture
(D) Hemothorax contusion
(E) Pneumonia

16. A 32-year-old female is stabbed in the right lateral neck $1 \mathrm{~cm}$ above the clavicle. There is an expanding hematoma in her neck, and she is having great difficulty speaking. Breath sounds are absent on the right. Subcutaneous air is noted in her neck. What is the next step in management?
(A) Intubation
(B) Chest tube placement
(C) Duplex ultrasound of the carotid
(D) Operative repair
(E) Esophagoscopy

17. A 25-year-old male arrives to the ED with a stab wound lateral to his umbilicus after being involved in a drunken fight at a local bar. You can smell alcohol on his breath, and he is uncooperative during the exam. His temperature is $99.2^{\circ} \mathrm{F}$, blood pressure is $90 / 60 \mathrm{mmHg}$, and pulse is $120 / \mathrm{min}$. His abdomen is soft, non-tender with no rebound or guarding. What is the most appropriate next step in management?
(A) Exploratory laparotomy
(B) Local wound exploration
(C) CT scan of the abdomen
(D) Serial physical examination
(E) FAST exam

18. In a patient presenting with acute limb ischemia in the right leg, what is the first structure to develop ischemic changes?
(A) Fat
(B) Nerve
(C) Muscle
(D) Skin
(E) Bone

19. A 60-year-old man is recovering in the ICU after being rescued from a fire within a restaurant kitchen. He was trapped for a prolonged period of time. He received deep partial and full-thickness burns in over $30 \%$ of his body. $\mathrm{He}$ has a past medical history of psoriasis controlled with topical steroids. On the seventh postoperative day, he becomes confused. His temperature is $96.1^{\circ} \mathrm{F}$, blood pressure is $98 / 72 \mathrm{mmHg}$, and pulse is $122 / \mathrm{min}$. His burn wounds have focal areas with a brown color. His laboratory examination demonstrates a white blood count of $14.7 \times 10^{3} / \mu \mathrm{L}$ (normal $4.1-10.9 \times 10^{3} / \mu \mathrm{L}$ ) and a serum glucose of $250 \mathrm{mg} / \mathrm{dL}$. What is the most likely etiology for this patient's acute condition?
(A) Intercompartmental fluid shift
(B) Adrenal insufficiency
(C) Alcohol withdrawal
(D) Carboxyhemoglobinemia
(E) Inflammation

20. A 30-year-old unrestrained driver is brought in by paramedics after a high-speed MVC. In the ED, his heart rate is $110 / \mathrm{min}$, blood pressure is $104 / 75 \mathrm{mmHg}$ and decreases to $92 / 68 \mathrm{mmHg}$ during inspiration. His tachycardia and hypotension persist despite aggressive fluid resuscitation. He appears pale, and his neck veins are distended. He has multiple bruises on his chest and abdomen. His chest $\mathrm{x}$-ray is unremarkable. What is the most likely diagnosis?

(A) Aortic transection

(B) Cardiac tamponade 

(C) Severe lung contusion
(D) Tension pneumothorax
(E) Diaphragm injury

21. A 38-year-old obese construction worker arrives to the trauma bay after accidentally getting struck by a bulldozer at his job site. In the ED, his mental status is altered, with a GCS of 10 . His blood pressure is $80 / 66 \mathrm{mmHg}$ with a pulse of $112 / \mathrm{min}$. He is given 2 liters of intravenous fluids, but his blood pressure and pulse remain the same. A FAST exam is inconclusive. A portable chest $\mathrm{x}$-ray is negative, and a pelvic $\mathrm{x}$-ray demonstrates bilateral pubic rami fractures. What is the best next step in management?

(A) Diagnostic peritoneal lavage (DPL)

(B) Pelvic angiography with possible embolization

(C) Exploratory laparotomy

(D) Head CT scan

(E) Abdominal CT scan

22. A 25-year-old male is at a pool party and is heavily intoxicated. He dives into the shallow end of the pool and is subsequently found to be floating face down in the pool. He is rushed to the ED by paramedics in a cervical collar. In the ED he opens his eyes, nods his head appropriately to questions, and his pupils are equally round and reactive to light. However, he is not moving his arms or legs. There is no evidence of external bleeding. His blood pressure is $85 / 45 \mathrm{mmHg}$, and his heart rate is $70 / \mathrm{min}$. Which of the following would most likely be seen in association with the injury described?
(A) Low cardiac output
(B) Elevated SVR
(C) Priapism
(D) Parasympathetic blockade
(E) Thoracic spine injury

23. A construction worker is digging a trench when he cuts his arm on a rusty nail in the soil. He is 45 years old and has not been to the doctor since he was a teenager, but he is confident he received all of his vaccinations up to age 18. What is the next step in treatment?
(A) Tetanus immunoglobulin only
(B) Tetanus vaccination only
(C) Tetanus immunoglobulin and vaccination
(D) Primary wound closure
(E) Clindamycin for 3 weeks

24. A 7-year-old boy presents to his pediatrician with a tense, painful, weak, and shortened forearm with a clawlike deformity of the hand. The mother states that 1 year earlier, the child fell backwards on his outstretched hand and suffered a supracondylar fracture that was treated with closed reduction and casting. The most likely explanation for the current physical exam findings is:
(A) Nerve entrapment
(B) Suppurative tenosynovitis
(C) Ischemia/necrosis of forearm muscles
(D) Complex regional pain syndrome
(E) Improperly reduced fracture

25. A 65-year-old former firefighter arrives for follow-up for chronic wound in his right leg from a burn he suffered 25 years earlier. The wound has failed to heal despite repeat skin grafting. Recently, the wound has become more painful and larger, measuring $2 \times 2 \mathrm{~cm}$, and continuously drains. Multiple biopsies of the wound are taken. Which of the following is the most important contributing factor to this patient's presenting condition?
(A) Radiation exposure
(B) Excess UV light exposure
(C) Work-related exposure to heavy metals
(D) Genetic predisposition
(E) Chronic inflammation

26. A 18-year-old man arrives to the ED combative and with severe shortness of breath, after suffering a stab wound to the chest. His blood pressure is $94 / 76 \mathrm{mmHg}$ with a pulse of $120 / \mathrm{min}$ and respiratory rate of $28 / \mathrm{min}$. Physical exam reveals a $2 \mathrm{~cm}$ stab wound on the left chest. Lung fields on the left have decreased breath sounds and are hyperresonant to percussion. His neck veins are distended. A needle is placed in the left second intercostal midclavicular line and aspirated until a gush of air is heard escaping the chest wall. A liter of normal saline is given, and blood pressure improves to $120 / 70 \mathrm{mmHg}$, and pulse decreases to $100 / \mathrm{min}$. What is the best next step in management?
(A) Transfuse O-negative blood
(B) Tube thoracostomy
(C) Chest X-ray
(D) CT scan of the chest
(E) Transport to the operating room for a thoracotomy

27. A 25-year-old male suffers a GSW to his right midthigh. On physical examination, there is no hematoma, no palpable thrill, and no bleeding from the wound. $\mathrm{He}$ has diminished but present pedal pulses on the right and normal pulses on the left. Neurological exam is normal. Ankle-brachial index on the right is 0.8 and 1.0 on the left. What is the next step in the management?
(A) Surgical exploration of the leg
(B) CT angiography
(C) Formal angiography
(D) Observation
(E) Systemic heparinization 
28. A 58-year-old intoxicated homeless man arrives to the ED after getting struck by an auto. His blood pressure on arrival was $98 / 55 \mathrm{mmHg}$ with a pulse of $120 / \mathrm{min}$. Following fluid resuscitation, his blood pressure increases to $120 / 70 \mathrm{mmHg}$, and pulse decreases to $80 / \mathrm{min}$. His abdomen is distended and mildly tender, and he has no obvious source of blood loss. A CT scan of the abdomen and pelvis shows no intraperitoneal fluid, but demonstrates bilateral pelvic fractures and a large pelvic fluid collection adjacent to the fracture with a contrast blush within it. What is the best next step in management?
(A) Military anti-shock trousers (MAST)
(B) External pelvic fixation
(C) Open reduction, internal fixation of pelvic fracture
(D) Exploratory laparotomy
(E) Emergency angiography with embolization

29. A 30-year-old male presents to the ED with a GSW to the right chest, just above his right nipple. In the ED, he complains of shortness of breath and severe right chest pain. His blood pressure is $110 / 70 \mathrm{mmHg}$, heart rate is $100 / \mathrm{min}$, and respiratory rate is $20 / \mathrm{min}$. On physical examination, his breath sounds are slightly diminished on the right. The trachea is midline. Neck veins are flat. The abdomen is non-tender. Upper extremity pulses are equal. A chest $\mathrm{x}$-ray demonstrates a moderate right hemo- and pneumothorax. The bullet is seen in the upper chest. A chest tube is inserted into the right chest with an immediate output of $500 \mathrm{cc}$ of dark blood, and after which the bleeding appears to slow down. What is the next step in the management?
(A) Exploratory right thoracotomy
(B) Video-assisted thoracoscopic surgery (VATS)
(C) Admit to ICU for observation
(D) Repeat chest X-ray
(E) CT of the chest and abdomen

30. A 28-year-old man arrives to the ED following a highspeed MVC. He is in severe pain and breathing rapid shallow breaths. His blood pressure is $80 / 60 \mathrm{mmHg}$, heart rate is $120 / \mathrm{min}$, and respiratory rate is $30 / \mathrm{min}$. A segment of his right anterolateral chest wall exhibits paradoxical inward motion on inspiration. Despite supplemental oxygen, the respiratory rate remains the same. Breath sounds are equal bilaterally. The trachea is midline. What is the next best step in management?
(A) Two liter bolus of normal saline
(B) Insert a right needle thoracostomy
(C) Endotracheal intubation
(D) Transfuse two units of O-negative blood
(E) Place tube thoracostomy (chest tube) on the right

31. Which of the following parameters would be most consistent with acute carbon monoxide poisoning?
(A) Hemoglobin decreases
(B) $\mathrm{PaO}_{2}$ decreases
(C) Oxygen content of blood decreases
(D) Oxidized hemoglobin increases
(E) Increased alveolar ventilation

32. Which of the following clinical scenarios is the best indication for four-compartment lower extremity fasciotomies?

(A) After a crush injury with an open tibia and fibula fracture

(B) After successful revascularization of a leg that was ischemic for $6 \mathrm{~h}$

(C) After repair of a femoral artery injury due to a GSW to the leg

(D) After repair of a combined popliteal artery and vein injury due to a gunshot injury

(E) After an electrical burn, with a tensely swollen and tender leg and numbness in the first web space 


\section{Answers}

\section{Answer D}

The FAST (focused abdominal sonogram for trauma) scan is a bedside ultrasound that is used to detect free fluid in the peritoneal cavity, around the pericardium, and in the thorax. The four areas of focus in a FAST exam are the hepatorenal space $(C)$, perisplenic space $(E)$, pouch of Douglas/rectovesical pouch, and pericardial space. FAST exam cannot distinguish blood from ascites and/or enteric content, is unable to detect retroperitoneal bleeds (from, for instance, a pelvic fracture), and is often times limited by obesity. FAST exam will be able to detect bowel perforation if there is free fluid and only if the bowel is within the peritoneum (so would miss injuries to parts of the duodenum, posterior walls of cecum, sigmoid). For detecting pericardial effusion (A), the sensitivity approaches nearly $100 \%$. Although bilateral pneumothoraces may limit comparison of sides, a single pneumothorax (B) has a sensitivity of $95 \%$ and specificity approaching $100 \%$.

\section{Answer C}

Penetrating neck trauma may result in injury to major blood vessels, the pharynx, esophagus, trachea, and/or cervical spine. Immediate surgical exploration (A) would be indicated if there were hard signs of vascular injury such as a pulsatile bleeding from the wound or rapidly expanding hematoma (the latter only after intubation $\{\mathrm{D}\}$ first to prevent airway compression). In the absence of hard signs of vascular injury, immediate surgical exploration is not necessary. Since physical examination is unreliable in terms of ruling out major injury, further imaging with CT angiogram (C) should be obtained. CT angiogram has largely replaced formal angiography $(\mathrm{E})$ which was once considered the gold standard. Formal angiogram is invasive (requires a femoral artery catheterization), time consuming, costly, and is only useful to rule out arterial injuries. Wound closure (B) would only be appropriate for injuries that do not penetrate the platysma.

\section{Answer B}

In a patient presenting with hypotension, distended neck veins, and muffled heart sounds (Beck's triad) following a stab wound to the chest, the most likely diagnosis is cardiac tamponade. The first sign in cardiac tamponade is impaired diastolic filling, which compromises cardiac output, and ultimately results in hypotension and distended neck veins (D, E). Electrical alternans (B) is characterized by varying alterations in the amplitude of the QRS complex between beats. It can occur in various other conditions and is not always present in patients with cardiac tamponade. Radiographic images are often negative initially, but some may develop the characteristic "water-bottle" shape later in the course of the disease $(\mathrm{C})$.

\section{Answer C}

Many worried pregnant patients arrive to the ED following minor trauma. Most patients do not have any significant clinical findings. Her nonstress test showed a normal strip. The criteria to discharge pregnant patients following minor trauma include contractions no more than every $10 \mathrm{~min}$, no vaginal bleeding, no abdominal pain, and a normal fetal heart tracing. This patient meets the discharge criteria and does not need to be monitored overnight (A). Biophysical profile (B) is indicated in patients with an abnormal nonstress test. CT of the abdomen (D) would be inappropriate in a pregnant patient because of the high radiation risk to the fetus. Although there have been no ill effects reported from MRI use during pregnancy, there are no indications to warrant MRI use in this patient $(\mathrm{E})$.

\section{Answer B}

Do not forget the ABCs of trauma. The airway should always be addressed first in the primary survey. Burn victims are at high risk for respiratory compromise since the supraglottic airway is susceptible to direct thermal injury and does not have the protection afforded to the infraglottic airway via the reflexive closure of vocal cords to intense heat. Circumferential burns of the neck further increase the risk of respiratory compromise by way of inelastic, circumferential eschars that may constrict the airway. Endotracheal intubation should be performed for all burn patients with acute respiratory distress, circumferential neck burns, full-thickness burns of the face or orpharynx, supraglottic edema, and progressive hoarseness, stridor, or wheezing. Broadspectrum antibiotics (A) are not routinely recommended for the management of burn victims. Burn patients are also at risk for severe intravascular collapse and require significant volume replacement with IV fluid resuscitation (C). However, this should be addressed after securing the airway. Premature ventricular contractions are usually benign (D). If the patient did not have indications for immediate intubation (circumferential neck burn), bronchoscopy (E) would be indicated in the presence of singed nasal hairs and carbonaceous sputum to determine the presence of thermal damage to the airway.

\section{Answer A}

High-energy rapid deceleration chest trauma is most commonly caused by a fall from greater than two stories or from a motor vehicle accident (e.g., steering wheel striking the chest). This mechanism of injury is known to cause aortic injuries which may lead to aortic transection, and ultimately death. Autopsy studies of aviation accidents demonstrate that more than $30 \%$ of deaths are due to aortic transection. Overall, immediate mortality is greater than $70 \%$. The majority of patients die instantly of exsanguination. Of those who survive, $49 \%$ will die within $24 \mathrm{~h}$. Patients will present 
with a widened mediastinum, deviation of the trachea to the right, and left-sided hemothorax on chest radiographs. They may also have fractures of bones (e.g., first rib, sternum, scapula) that are uncommonly broken as high energy is required to break them. The aortic tear is usually at the ligamentum arteriosum, located just distal to the subclavian take off, as the aortic arch is relatively fixed to that point. CT angiogram can confirm the diagnosis, and definitive management includes operative repair. Although a ruptured spleen (D) can lead to significant blood loss, instant death is highly unlikely. The remaining choices $(\mathrm{B}, \mathrm{C}, \mathrm{E})$ can all cause instant death, but they occur in less frequency than thoracic aortic transection with this mechanism of injury. Abdominal aortic transection is extremely rare following blunt trauma as it is more mobile than the thoracic aorta.

\section{Answer D}

A dislocated limb has the potential of compromising arterial blood flow. As such prompt reduction is essential. However, prior to reduction, the first step is to obtain a plain film of the limb to confirm the dislocation and to rule out associated fractures. Following reduction, a postreduction film is needed to confirm proper alignment. Fasciotomy (A) would be indicated if there is concern for compartment syndrome (pain in calf muscles on passive motion, tense swelling, paresthesias); however, reduction of a dislocated knee would still take priority. CT angiography (B) would be performed after reduction if there is concern for arterial injury (ankle-brachial index $<0.9$ ). Heparinization (C) would be initiated after limb ischemia is diagnosed (e.g., cold, pulseless limb). MRI of the knee $(\mathrm{E})$ is seldom indicated in the acute setting for knee injuries.

\section{Answer $\mathrm{E}$}

The diagnosis of isolated pancreatic injury is often delayed as it is notoriously known to be missed initially on CT. If there is no associated splenic injury to cause bleeding or bowel injury to cause peritonitis, initial physical examination findings may be unremarkable. In addition, a serum amylase level (A) is neither specific nor sensitive for pancreatic injury. However, if there is pancreatic duct disruption, the release of enzymes will eventually lead to symptoms as in the patient presented above. Surgery is recommended for such major injuries. Minor pancreatic injuries without pancreatic duct disruption can be managed nonoperatively. In such cases, ERCP (B) is more sensitive and specific than MRCP (D) for ductal injury. CT-guided drainage (C) will not address the underlying pancreatic injury and would not be appropriate for this patient.

\section{Answer B}

The patient has sustained a blunt injury to the carotid artery as evidenced by a dissection in the left internal carotid artery.
Such an injury should be suspected whenever there is highenergy force to the head and/or neck. He is exhibiting evidence of Horner's syndrome (ptosis, meiosis, anhidrosis), as sympathetic nerve fibers can be interrupted with carotid injury. A dissection is a partial-thickness tear in an artery that begins in the intima and extends into the media. It can narrow or occlude the lumen. Most blunt carotid injuries are managed nonoperatively with anticoagulation (provided there is no contraindication). Thus observation (E) alone would be inappropriate for such a patient. Since the dissection extends to the base of the skull, it would be impossible to access and repair through a standard neck incision (A). Conservative management using heparin (B) is the most appropriate option and has been shown to reduce or prevent cerebral infarction in patients with blunt carotid injury. Carotid stenting $(\mathrm{C})$ has a risk of causing a stroke and would not be appropriate for a dissection that extends to the base of the skull. Thrombolysis (D) is contraindicated in a patient with a carotid dissection and in patients with trauma causing acute vascular injury.

\section{Answer $C$}

Immediate exploratory laparotomy is recommended in the majority of patients with a GSW to the abdomen, particularly if the patient is hemodynamically unstable, has evidence of peritonitis, or has bowel evisceration. However, cooperative patients with gunshot wounds (GSW) to the abdomen that are hemodynamically stable, with no evidence of peritonitis, are candidates for nonoperative management (NOM). They should be evaluated further for injuries requiring surgical repair with an abdominal CT scan. This approach may avoid an unnecessary exploratory laparotomy (B) that carries significant morbidity. CT scan should still be done even for patients with wounds that appear to only be superficial. If the CT scan is normal, the patient can be managed with serial physical exams (A) and serial laboratory exams (e.g., white blood count). NGT can help identify gastric injuries, while rectal examination can help identify rectal or colon penetration by the bullet. Though occasionally utilized for penetrating trauma, DPL and $\operatorname{FAST}(\mathrm{D}, \mathrm{E})$ are more appropriate for blunt trauma.

\section{Answer A}

It is important to note that acute limb ischemia (in this instance due to embolization of atrial thrombus secondary to atrial fibrillation), followed by reperfusion, is a well-recognized risk for the subsequent development of compartment syndrome. Ischemia-reperfusion results in an increase in vascular permeability to plasma proteins and progressive interstitial edema. This leads to an increase in interstitial pressure. When interstitial pressure exceeds capillary perfusion pressure, muscle ischemia and necrosis ensue. It is important to note that palpable pulses do not rule out compartment 
syndrome. Treatment is an emergent 4-compartment fasciotomy. The lymph system (E) is not involved in the development of acute compartment syndrome. A recurrent embolus (B) would not be expected to present with a swollen leg and palpable distal pulses. DVT (C) can present with calf tenderness that is worsened with passive extension (Homan sign). However, the temporal relation to his presenting problem and the physical exam findings are more supportive for compartment syndrome. Atherosclerotic plaque (D) would be expected in a patient presenting with claudication secondary to peripheral arterial disease.

\section{Answer D}

Infections in burn patients can be problematic for multiple reasons. It may delay wound healing, encourages scarring, and can result in bacteremia which may lead to sepsis. Pseudomonas aeruginosa is a gram-negative bacillus and is considered to be the most common cause of infections in burn patients. Methicillin-resistant Staphylococcus aureus (A) is also commonly seen in burn patients and difficult to treat due to a large number of virulence factors. Streptococcus pyogenes (B) is more of a concern in pediatric burn patients because they may have colonization of Streptococcus pyogenes in their oropharynx. Streptococcus agalactiae (C) is not an organism thought to infect burn patients. This organism can colonize the genitourinary tract and be transmitted to the neonate during birth which may result in bacteremia, pneumonia, or meningitis. Fungal infections tend to occur in burn patients during the later stages of recovery because by this time the majority of bacteria have been eliminated by the use of antibiotics. The most common cause of fungal infection in burn patients is by Candida albicans $(\mathrm{E})$.

\section{Answer B}

Duodenal injury following blunt abdominal trauma is rare. When it does occur, it is usually accompanied by other abdominal injuries. Isolated duodenal injuries are even more uncommon. In children, they have classically been reported following a direct blow to the epigastrium such as a bicycle handlebar injury. The retroperitoneal location of some portions of the duodenum may lead to a delay in diagnosis, as enteric contents spilling from the injury may not cause peritonitis. Contrast-enhanced CT scan of the abdomen can help confirm the diagnosis by detecting extravasation of oral contrast, the presence of retroperitoneal air, or a paraduodenal hematoma. Some duodenal injuries can be managed nonoperatively. Specifically, a duodenal wall hematoma, without contrast extravasation does not require surgery. On the other hand, the presence of contrast extravasation confirms a full-thickness injury that mandates exploratory laparotomy. Depending on the extent of injury, primary repair can be performed. Because of the close relationship of the duodenum to the pancreas and the bile duct, resection of the duodenum is often not possible. Upper endoscopy (D) would be contraindicated in the presence of bowel perforation. CT-guided drainage (E) will not address the underlying duodenal injury and would not be appropriate for this patient. Laparoscopy (A) would not likely be able to adequately assess and repair the duodenal injury.

\section{Answer C}

This patient has evidence of compartment syndrome that has led to muscle necrosis (as evidenced by high CPKs and hyperkalemia). Though compartment syndrome is mostly thought of as caused by severe bleeding after trauma, there are many other causes. In this case, it occurred secondary to prolonged compression of the forearm muscles due to his alcohol and drug binge. This resulted in ischemia, followed by reperfusion, and then swelling and death of the muscles. An alcohol binge can also lead to Saturday night palsy, a colloquial term referring to radial neuropathy from falling asleep with one's arm hanging over a park bench (compressing the spiral groove which houses part of the radial nerve). Hyperkalemia is a known complication of muscle necrosis from compartment syndrome and can lead to peaked $\mathrm{T}$ waves, and if left untreated, fatal arrhythmias. Although all the options listed (A, B, D, E) are appropriate management options for hyperkalemia, calcium gluconate should be administered first to stabilize cardiac myocytes and prevent further damage, particularly because the electrolyte imbalance has already begun to affect the heart (e.g., peaked T waves).

\section{Answer B}

The key to the diagnosis is the history of trauma combined with the chest $\mathrm{x}$-ray. On initial inspection, the chest $\mathrm{x}$-ray could be confused with a hemothorax (D) or pneumonia (E). However, the presence of multiple air pockets within the left lung field indicates that there are loops of bowel in the left chest, likely due to a traumatic left-sided diaphragmatic hernia. Traumatic diaphragmatic hernia (TDH) can occur following blunt abdominal trauma secondary to a sudden increase in intra-abdominal pressure. Diagnosis is frequently delayed since patients may be asymptomatic immediately following the traumatic episode. The stomach and colon are the most frequently herniated structures. Patients with TDH can present with both GI and respiratory symptoms. Gastroenteritis (A) is unlikely to present with an increased respiratory rate or an abnormal chest $\mathrm{x}$-ray. Following blunt trauma, patients can very rarely present with a delayed splenic rupture, and this could cause a reactive left pleural effusion. However, once again, this would not cause loops of the bowel in the chest.

\section{Answer A}

This patient has likely sustained damage to several structures of zone 1 of the neck. The first steps in management are 
always ABC. Given that there is an expanding hematoma and she is having difficulty speaking, there is concern that her airway is compromised, so she should be intubated. Since the apices of the lungs are contained within zone 1 of the neck, and she has absent breath sounds, she likely has a pneumothorax and will also need a chest tube (B). Duplex ultrasound of the carotid $(\mathrm{C})$ is not necessary since there is a hard sign of vascular injury. The patient requires operative repair (D), but the airway should be protected first. This patient may have sustained esophageal injury that will require repair as well, but esophagoscopy (E) should not be performed since she has a hard sign of vascular injury.

\section{Answer A}

This patient has a penetrating abdominal wound which is concerning for an intraperitoneal injury. Immediate exploratory laparotomy is recommended in patients with a penetrating injury to the abdomen if the patient is hemodynamically unstable, has evidence of peritonitis, has bowel evisceration, or is uncooperative (e.g., intoxicated). Further work-up (B-E) can be considered for patients that are hemodynamically stable, with no evidence of peritonitis.

\section{Answer $C$}

The muscle is the first structure to be affected by ischemic changes in acute limb ischemia, and since it is the primary mass of the tissue in the extremity, the extent and duration of muscle damage are the most critical aspects of limb reperfusion syndrome and subsequent compartment syndrome. The muscle can be tolerant of ischemia for up to $4 \mathrm{~h}$. Irreversible nerve damage (B) occurs after $8 \mathrm{~h}$ of ischemia. Fat (A) changes remain reversible for up to $13 \mathrm{~h}$, the skin (D) up to $24 \mathrm{~h}$, while the bone (E) damage does not typically occur until after 4 days of ischemia.

\section{Answer E}

Patients with severe burns are at increased risk of burn wound sepsis. This patient has hypothermia, leukocytosis, and tachycardia. Thus he meets the diagnostic criteria for systemic inflammatory response syndrome (SIRS). Patients must have two of the following four in order to be diagnosed with SIRS: fever of more than $100.4^{\circ} \mathrm{F}$ or less than $96.8^{\circ} \mathrm{F}$, heart rate of more than 90 , respiratory rate of more than 20 , or white blood count of $>12,000 / \mu \mathrm{L}$ or $<4,000 / \mu \mathrm{L}$. SIRS due to an infection is called sepsis and can manifest with confusion or altered levels of consciousness (i.e., end-organ damage). Burn patients in particular are susceptible to bacterial infections. Changes in the color of the burn wound (to red, brown, or black) should raise suspicion for wound sepsis. Intercompartmental fluid shifts (A), or third spacing, occur when fluid that accumulated in the interstitium of tissues during the postoperative period shifts back into the intravascular space, typically on postoperative day three. This will present with a patient that appears to be fluid overloaded. Tertiary corticoadrenal insufficiency (B) should always be on the differential for patients with long-term steroid use that develop hypotension. This occurs because of insufficient corticotropin-releasing hormone secretion by the hypothalamus. However, the risk is less in patients using topical steroids because of its decreased potency and limited systemic exposure. In addition, hypothermia would not be expected with adrenal insufficiency. Alcohol withdrawal (C) would be expected to begin within $24 \mathrm{~h}$ of the last drink (not 7 days later). It can present with a wide range of symptoms including tremulousness, insomnia, anxiety, diaphoresis, and autonomic hyperactivity. Burn patients are at risk of carbon monoxide poisoning (D), particularly when they are confined to a close space. However, carbon monoxide poisoning will present acutely (not 7 days later) with headaches, dizziness, and nausea.

\section{Answer B}

Patients with blunt chest trauma that present with persistent hypotension, tachycardia, and elevated JVP should be suspected of having an injury to the heart. Furthermore, this patient had a drop $\geq 10 \mathrm{mmHg}$ in systolic blood pressure during inspiration (pulsus paradoxus) which supports a diagnosis of cardiac tamponade. Although cardiac tamponade classically causes a globular appearance of the heart on CXR, the cardiac silhouette may be normal. A lung contusion (C) would cause respiratory distress but not features of tamponade. An aortic transection (A) presents with a wide mediastinum and would not cause neck vein distention. Tension pneumothorax (D) may have distended neck veins, but the collapsed lung would be apparent on CXR combined with tracheal deviation. Diaphragmatic injury (D) can occur following blunt abdominal trauma and often present with GI and respiratory complaints though they may initially be asymptomatic.

\section{Answer A}

Hypotension after blunt trauma should be considered due to hemorrhage until proven otherwise. Head injury should not be considered the source of hypotension. The most likely sources of bleeding are the abdomen, pelvis, and chest. However, major chest bleeding has been ruled out by the negative CXR. In the stable patient, an abdominal CT (E) is the best test to rule out bleeding. However, the patient's hemodynamic instability precludes such a study. FAST scan is the test of choice in the unstable patient, but its utility is often limited in obese patients because of poor image quality. In equivocal cases, the next best choice is to perform a DPL to detect free fluid in the peritoneum, which would be an indication for exploratory laparotomy. Proceeding directly to exploratory laparotomy (C) would be appropriate if the patient manifested peritoneal signs. However, his altered mental status precludes a proper physical examination. 
Pelvic bleeding is another potential source of bleeding, although pubic rami fractures rarely cause major bleeding (more likely with fractures of the posterior pelvis). If the DPL was negative, one would then pursue pelvic angiography (B) to rule out pelvic bleeding. Given the GCS of 10, a head CT (D) is indicated, but this would not take precedence over identifying the source of hemorrhagic shock first.

\section{Answer $C$}

The patient is displaying evidence of neurogenic shock with hypotension and an inappropriately normal heart rate (or bradycardia). Neurogenic shock is associated with a high cervical spinal cord injury (not thoracic spine injury $\{E\}$ ). Priapism (a sustained erection due to unopposed parasympathetic tone) is often a presenting sign of acute spinal cord injury. Neurogenic shock would be expected to present with a normal/high cardiac output (A), decreased SVR (B), and sympathetic blockade (D). Treatment is with intravenous fluids and if needed, pressor support (with an alpha agent for vasoconstriction)

\section{Answer B}

This patient has a dirty wound, but has likely had all three tetanus vaccinations. Based on the table below, the correct treatment is tetanus vaccination only. Antibiotics (E) are not indicated since the patient is not infected. Since this is a dirty wound, primary closure (D) may not be attempted in this case, and the wound may be packed instead (Table A.1).

\section{Answer C}

Children with supracondylar fractures are at risk for acute compartment syndrome. There are three mechanisms as to why this occurs: (1) the fracture is associated with an often unrecognized brachial artery injury that leads to ischemia in the compartments of the arm; (2) if the subsequent cast is placed too tightly, this may contribute to compartment syndrome; (3) initial bleeding and muscle damage/edema causes high pressures in the compartments of the arm leading to compartment syndrome. Compartment syndrome presents with the 6 Ps (pain, pallor, pulselessness, paresthesias, paralysis, and poikilothermia). Treatment is fasciotomy. Volkmann's contracture is the manifestation of unrecognized and untreated compartment syndrome. This occurs because

Table A.1 Tetanus vaccination

\begin{tabular}{lll}
\hline $\begin{array}{l}\text { History of TT } \\
\text { vaccination }\end{array}$ & Clean wounds & Dirty wounds \\
\hline$<3$ doses & All should receive TT & $\begin{array}{l}\text { All should receive both TT } \\
\text { and TIG }\end{array}$ \\
\hline$\geq 3$ doses & $\begin{array}{l}\text { Should receive TT only } \\
\text { if the last dose was }>10 \\
\text { years go }\end{array}$ & $\begin{array}{l}\text { Should receive TT only if } \\
\text { last dose was }>5 \text { years ago }\end{array}$ \\
\hline
\end{tabular}

$T T$ tetanus toxoid, $T I G$ tetanus immunoglobulin prolonged ischemia can lead to muscle death and subsequent fibrotic changes within the tissue. Volkmann's contracture presents with a tense, painful, weak, and shortened forearm with a claw-like deformity of the hand. Nerve entrapment (A) is more likely to present acutely after the injury and will have deficits consistent with the distribution of a particular nerve. Suppurative tenosynovitis (B) is characterized by the four cardinal signs (Kanaval signs): flexor tendon sheath tenderness, fusiform swelling (sausage-shaped digits), pain with passive extension, and a semi-flexed posture of the involved digit. Complex regional pain syndrome (D) is a poorly understood phenomenon that occurs in patients that have had a crushing or soft tissue injury, typically to the distal extremities. They can present within days or months with intermittent pain, difficulty using the extremity, neglect-like symptoms, and rapid fatigability. An improperly reduced fracture would have been recognized earlier and corrected and would be unlikely to result in the deficits seen in this patient.

\section{Answer E}

Cutaneous squamous cell carcinoma arising from a chronic non-healing wound (such as a burn) is known as Marjolin's ulcer. Although all the answer choices (A-D) are considered independent risk factors for skin cancer, chronic inflammation is the most important contributing factor in Marjolin's ulcer and can be seen in burn wounds, scars, chronic ulcers, or sinus tracts. Carcinoma develops on average 20-30 years after the original burn. All chronic wounds that fail to heal after a long period should undergo a skin biopsy to rule out malignancy.

\section{Answer B}

This patient has a left-sided tension pneumothorax as confirmed by hypotension, distended neck veins, decreased breath sounds, and hyperresonant left chest. Immediate treatment is with needle thoracostomy, allowing for immediate thoracic decompression. This is preferred in the setting of a tension pneumothorax as it is faster than a chest tube, but provides only temporary relief. All these patients require a tube thoracostomy (chest tube) immediately following needle thoracostomy. Operative management $(\mathrm{E})$ is not routinely indicated for patients with tension pneumothorax as needle decompression and subsequent tube thoracostomy are able to resolve most cases. If he had a significant hemothorax that continued to hemorrhage despite tube thoracostomy, surgical management could be considered as well as blood products (A). Tension pneumothorax is considered a clinical diagnosis, and confirmation with imaging $(\mathrm{C}, \mathrm{D})$ is not recommended as it delays definitive care in the unstable patient.

\section{Answer B}

Penetrating trauma to the extremities should be assessed for neurovascular injuries. Prompt surgical exploration (A) 
would be indicated if the patient had hard signs of vascular injury (e.g., pulsatile bleeding, expanding hematoma). In the absence of such signs, an ABI should be checked. If the ABI is $<0.9$, suspicion for an arterial injury is high, and as such, imaging with CT angiography is the most appropriate management option. Formal angiography (C) can be considered if $\mathrm{CT}$ results are equivocal. Observation would be appropriate if he had a normal ABI. Systemic heparinization is sometimes used during the course of arterial repair if the injury led to thrombosis and an interposition vein graft is used.

\section{Answer E}

This patient's mechanism of injury and blood pressure drop are highly suggestive of hemorrhagic shock. Given that the patient responded well to IV fluids, it is appropriate to obtain CT imaging to look for the source of bleeding. If the source was intra-abdominal bleeding, the next step would be exploratory laparotomy (D). However, the CT indicates that the source is pelvic bleeding, likely from the pelvic fracture. Such bleeding is best managed via emergent pelvic angiography, which could be diagnostic and therapeutic (with embolization). MAST (A) suits were at one time popular as the compression was thought to tamponade bleeding. However, they have not been shown to be effective. External pelvic fixation (B) can reduce and stabilize fractures and thus lead to a slowing of bleeding, but is not considered as effective as angiographic emoblization. Open reduction, internal fixation $(\mathrm{C})$ is the definitive treatment for a pelvic fracture. But given the technical difficulty and long length of such an operation, it is not recommended acutely, and especially not in someone who is actively bleeding. Pelvic packing is emerging as an alternative to angiography for pelvic bleeding.

\section{Answer D}

This patient presents with a right hemo- and pneumothorax, and tube thoracostomy was able to evacuate $500 \mathrm{cc}$ of dark blood. The most appropriate next step in management is to perform a repeat chest $\mathrm{x}$-ray to ensure that the tube thoracostomy is in the right position and that the hemo- and pneumothorax have resolved. Exploratory right thoracotomy (A) would be indicated only if the initial output after chest tube placement was $>1,500 \mathrm{cc}$ or if the patient continued to bleed briskly ( $>200 \mathrm{cc} / \mathrm{h}$ for $3 \mathrm{~h}$ ). VATS (D) is indicated if the chest tube has inadequately drained the hemothorax. But such a residual hemothorax would be drained via VATS only after failure of a second chest tube and only after waiting a few days (not acutely). CT of the chest is generally not needed if the CXR shows that the hemothorax is resolved, and CT of the abdomen (E) is unnecessary at this time as the bullet entered just above the nipple (and thus above the diaphragm) and is visualized in the chest, thus sparing the abdominal cavity.

\section{Answer C}

This is concerning for a flail chest, most commonly caused by blunt trauma. Although the diagnosis is made clinically with a paradoxical inward motion of the chest wall during inspiration, it is supported by imaging studies demonstrating two or more consecutive ribs broken at two or more sites. The primary morbidity related to flail chest is the frequent underlying pulmonary contusion that accompanies it and compromises adequate respiration. Furthermore, severe pain may also affect respiration. Always start with the ABCs of trauma. The best course of management for the above patient (given the marked tachypnea and flail chest) is to first ensure an airway with endotracheal intubation. This can be followed by two large bore IVs and fluids (A). Blood products (D) may be needed if he does not respond to fluids and continues to remain hemodynamically unstable. There is no indication for a needle thoracostomy (B) or chest tube given that the breath sounds are equal. Chest tube (E) may be indicated if the patient had a concurrent pneumothorax on subsequent CXR.

\section{Answer C}

Acute carbon monoxide (CO) poisoning affects the organs with the highest oxygen demand first. Patients will present in the early stages with neurologic complaints (e.g., headaches, dizziness, confusion) and cardiac symptoms (e.g., chest pain, arrhythmias). All these patients should be started on $100 \%$ oxygen via nonrebreather facemask. CO has nearly $250 \times$ more affinity for hemoglobin than oxygen. Thus the hemoglobin-oxygen dissociation curve shifts to the left, and more hemoglobin is bound by $\mathrm{CO}$ than it is by oxygen. This decreases both the hemoglobin saturation (of oxygen) and the oxygen content in the blood. The arterial partial pressure of oxygen is not affected in $\mathrm{CO}$ poisoning (B), and so a compensatory increased alveolar ventilation would not be expected (E). CO poisoning is not a consumptive or destructive process, and so hemoglobin would not be expected to change (A). Oxidized hemoglobin, also known as methemoglobin, has a higher affinity for cyanide, and so patients with cyanide poisoning are oftentimes given nitrates to induce the oxidization of hemoglobin to help bind the cyanide for renal clearance.

\section{Answer E}

Electrical burns are deceptive as at the skin level there may be a relatively minor burn wound. Yet, the electrical current can penetrate deep into the soft tissues, leading to extensive injury to the soft tissues and muscle. Thus electrical burns are associated with the development of compartment syndrome. The best indication for fasciotomy is in the presence of compartment syndrome. Choice $\mathrm{E}$ is the only choice in which there is an absolute indication for fasciotomy as the patient has clear evidence of compartment syndrome. Numbness of the first web space is the classic finding of 
anterior compartment syndrome, as the deep peroneal nerve travels within it, and it supplies sensation to the first web space. Options B, C, and D are relative indications for prophylactic fasciotomy, as they place the patient at increased risk of subsequently developing compartment syndrome, although prophylactic fasciotomies are controversial. A crush injury (A) by itself is not considered an indication for prophylactic fasciotomy. 


\section{Upper Gastrointestinal}

\section{Michael D. Sgroi, Brian R. Smith, Christian de Virgilio, Areg Grigorian, and Paul N. Frank}

\section{Questions}

1. A 60-year-old male presents with gnawing epigastric pain that has been present for 8 weeks. The pain seems to get worse with eating. He denies nausea, vomiting, or early satiety. He has lost $10 \mathrm{lb}$ and he attributes this to a poor appetite. He denies black or bloody stools. Physical examination is unremarkable. What is the next study to be ordered?
(A) Barium swallow
(B) CT scan
(C) EGD
(D) 6-week trial of proton pump inhibitor
(E) Testing for $H$. pylori

2. Which of the following is the strongest risk factor for gastric cancer?
(A) Type A blood
(B) H. pylori infection
(C) Smoking
(D) Familial adenomatous polyposis
(E) Family history

3. A 70-year-old male with a history of smoking presents with vague upper abdominal and epigastric pain and coffee ground emesis. On EGD, a small ulcer is found in the stomach, with evidence of a large submucosal mass underneath the ulcer. Biopsy of the area is negative. CT scan confirms a 4-cm homogeneous, well-circumscribed, submucosal mass in the greater curvature of the stomach. What does this mass most likely represent?
(A) Gastrointestinal stromal tumor
(B) Gastric adenocarcinoma
(C) Gastric lipoma
(D) Metastatic carcinoma
(E) Solitary fibrous tumor

4. A 65-year-old female presents with coffee ground emesis $\times 3$. She has had vague upper abdominal pain for the past 2 months, relieved by taking antacids. In addition, she reports an involuntary weight loss of $10 \mathrm{lb}$. She denies NSAID or alcohol use. On physical examination, her blood pressure is $110 / 70 \mathrm{mmHg}$ and HR is $80 /$ min. Abdominal examination reveals mild epigastric tenderness with no rebound or guarding. Laboratory values are significant for a $\mathrm{Hgb} / \mathrm{Hct}$ of $8.3 \mathrm{~g} / \mathrm{dL}$ (normal $12-15 \mathrm{~g} / \mathrm{dL}$ ) and $24 \%(36-44 \%$ ) with an MCV of $80 \mathrm{fL}$ (80-100 fL), total bilirubin of
$3.0 \mathrm{mg} / \mathrm{dL}(0.1-1.2 \mathrm{mg} / \mathrm{dl})$, alkaline phosphatase of $250 \mathrm{IU} / \mathrm{L}$ (33-131 IU/L), a GGT of $270 \mathrm{IU} / \mathrm{L}$ (6-37 IU/L), an ALT of $300 \mathrm{IU} / \mathrm{L}(<35 \mathrm{IU} / \mathrm{L})$, and an AST of $320 \mathrm{IU} / \mathrm{L}(<35 \mathrm{IU} / \mathrm{L})$. The most likely diagnosis is:
(A) Duodenal ulcer
(B) Gastric ulcer
(C) Pancreatic cancer
(D) Ampullary cancer
(E) Dieulafoy's lesion

5. A young man from Armenia arrives to the ED complaining of a progressively bloody cough. He reports night sweats and fevers for the past month. He has been in the United States for 1 week visiting family. As you begin examining him, he coughs up a massive amount $(200 \mathrm{ml})$ of bright red, foamy sputum and has difficulty speaking. Portable chest $\mathrm{x}$-ray shows multifocal patchy and cavitary opacities in the right upper lobe with mediastinal lymphadenopathy. Given his increased risk for asphyxiation, the patient is intubated and placed in a right lateral decubitus position. What is the next best step in management?
(A) Emergent thoracotomy in the OR
(B) Bronchoscopy
(C) Video-assisted thoracic surgery (VATS)
(D) Emergency arteriography
(E) INH, rifampin, ethambutol, and pyrazinamide

6. A 50-year-old male presents to the ED stating that he passed a large amount of maroon stool earlier in the day. $\mathrm{He}$ is currently not passing any stool. He has not vomited. He denies abdominal pain or weight loss. He also has a history of nosebleeds. He takes no medications and does not drink alcohol. Physical examination is only significant for small red nodules on his lips. Abdominal examination is unremarkable. Upper endoscopy is negative. Which of the following is most likely to localize the site of the GI bleeding?
(A) Capsule endoscopy
(B) CT angiogram
(C) Lower endoscopy
(D) Formal angiography
(E) Technetium-labeled red blood cell scan

7. A 50-year-old female presents with massive UGI bleeding. In the ED, she is actively vomiting large amounts of bright red blood. She appears to be lethargic and pale. Blood pressure is $70 / 50 \mathrm{mmHg}$, heart rate is $120 / \mathrm{min}$, and $\mathrm{RR}$ is $22 / \mathrm{min}$. The next step in the management is:

(A) Emergent upper endoscopy

(B) Immediate orotracheal intubation

(C) Place bed in reverse Trendelenburg position 
(D) Give 2 liter bolus of normal saline

(E) Give two units of O-negative blood

8. A 48-year-old male presents with vomiting of bright red blood. He states that he has had a 1 month history of epigastric pain that is relieved by eating food. He denies weight loss. He does not take any medications or drink alcohol. He is hemodynamically stable in the emergency department. Following resuscitation, the patient undergoes upper endoscopy which reveals a posterior ulcer in the proximal duodenum which is actively bleeding. Despite numerous attempts at cauterization and injection with epinephrine, the gastroenterologist reports that she cannot stop the bleeding and the blood pressure drops to $70 / 50 \mathrm{mmHg}$. The next step in the management is:

(A) Angiographic embolization

(B) Transfer to ICU for ongoing transfusion of blood

(C) Administer vasopressin

(D) Exploratory laparotomy

(E) Administer octreotide

9. A 45-year-old male with a history of alcohol abuse presents to the emergency department with an UGI bleed after a night of binge drinking. The patient reports that he repeatedly dry heaved, after which he began to note bright red blood in the vomitus. He is afebrile with normal vital signs. Upper endoscopy reveals a partial tear in the mucosa and submucosa of the stomach near the gastroesophageal junction. Which of the following is true about this condition?

(A) It is usually related to portal hypertension

(B) It is often associated with a left pleural effusion

(C) It is associated with $H$. pylori infection

(D) The bleeding is likely arising from the gastroduodenal artery

(E) The bleeding most often stops spontaneously

10. A 65-year-old male presents with melena. He reports no abdominal pain and no vomiting. He denies a prior similar history. In the ED, he is tachycardic to $120 / \mathrm{min}$ with a blood pressure of $112 / 80 \mathrm{mmHg}$. Hemoglobin and hematocrit are $9.2 \mathrm{~g} / \mathrm{dL}$ (normal $13-16 \mathrm{~g} / \mathrm{dL}$ ) and $28 \%$ $(40-52 \%)$, respectively. Past history is significant for an aortobifemoral bypass 5 years earlier for severe claudication. Nasogastric (NG) tube aspiration returns clear fluid. The patient is admitted to the ICU for resuscitation, the melena ceases, and the hematocrit stabilizes. Upper endoscopy is negative, with no stigmata of bleeding. Following a bowel prep, colonoscopy is also negative. CT scan of the abdomen reveals gas around the proximal aortic graft. Which of the following is true about this condition?
(A) Formal angiography is likely to demonstrate the source of bleeding

(B) Gas around the aortic graft is not considered pathologic

(C) The aortic graft will likely need to be removed.

(D) Rebleeding is rare

(E) This represents an occult bleed

11. What is the most common cause of an esophageal perforation?
(A) Boerhaave syndrome (spontaneous perforation)
(B) Iatrogenic
(C) Foreign body
(D) Esophageal cancer
(E) Trauma

12. Which of the following is true of Boerhaave syndrome?

(A) It most often presents with UGI bleeding

(B) It is most often caused by an iatrogenic injury

(C) It is a partial thickness tear of the esophagus

(D) It is the most lethal GI perforation

(E) Treatment consists of bowel rest and intravenous antibiotics

13. A 50-year-old Asian female complains of abdominal discomfort and weight loss. She has an EGD performed, and biopsy of a stomach lesion returns as mucosa-associated lymphoid tissue (MALT) lymphoma. Which of the following is the best treatment plan for this patient?

(A) Chemotherapy

(B) Radiation therapy

(C) Combined chemotherapy and radiation

(D) Clarithromycin, amoxicillin, and a proton pump inhibitor

(E) Gastric resection

14. Which of the following is the first step in the treatment of a patient with Boerhaave syndrome?

(A) Esophagectomy

(B) Gastric decompression

(C) Drainage of the mediastinum and pleura

(D) Primary repair of the perforation with advancement flap coverage

(E) Fluid resuscitation and IV antibiotics

15. A 65-year-old male presents with iron deficiency anemia. Fecal occult blood testing is positive. Upper endoscopy demonstrates an irregular ulcer in the body of the stomach. Biopsy confirms adenocarcinoma. Simultaneous endoscopic ultrasound (EUS) demonstrates that the mass has invaded into the submucosa. No enlarged nodes are 
seen around the lesion. Which of the following would be the next best step in the management?
(A) Proceed to gastric resection
(B) CT scan of the abdomen
(C) PET scan
(D) Laparoscopy
(E) Chemotherapy

16. Successful eradication of $H$. pylori is best documented by:
(A) EGD with biopsy
(B) Urea breath test
(C) Blood antibody test
(D) Stool antigen
(E) Documentation is unnecessary provided symptoms have resolved




\section{Answers}

\section{Answer $\mathrm{C}$}

Epigastric pain and symptoms of dyspepsia are extremely common. It is reasonable in most young patients to start with a short trial of proton pump inhibitors (D). Alternatively, clinicians can employ the test-and-treat strategy in which they test for $H$. pylori first (E). Testing should only be done if the clinician plans to offer treatment for a positive test. However, in patients $>55$ years or in those with ALARM Symptoms, Anemia (iron deficiency), Loss of weight, Anorexia, Recent onset of progressive symptoms, Melena/hematemesis, $S$ wallowing problems, upper endoscopy is required to rule out gastric cancer. CT scan (B) is usually performed after EGD to evaluate for distant metastasis. Operative resection should not be undertaken until the diagnosis is confirmed and the tumor is staged. Barium swallow (A) may identify gastric ulcers and infiltrating lesions, but it has a high falsenegative rate and does not permit biopsy. Barium swallow may also be used to evaluate dyspepsia in younger patients.

\section{Answer B}

H. pylori is the strongest established risk factor for gastric cancer worldwide. H. pylori infections lead to gastritis, and without eradication of the infection, inflammatory changes may eventually lead to dysplasia and metaplasia. All the remaining choices (A, C-E) increase one's risk of developing gastric cancer but are not considered to be as strong as $\mathrm{H}$. pylori.

\section{Answer A}

Gastrointestinal stromal tumors (GIST) are mesenchymal tumors that most often occur in the stomach and small intestine. As they grow, they can cause pressure necrosis, leading to erosion of the gastric mucosa and GI bleeding. Since the mass is submucosal, attempts at biopsy at the time of upper endoscopy are often negative. CT scan typically shows a well-circumscribed homogeneous mass. GIST are caused by a gain of function mutation (tumorigenesis) in the protooncogene c-KIT. Treatment is surgical resection, followed by imatinib mesylate, a selective tyrosine kinase inhibitor with action against mutant c-KIT. Gastric adenocarcinoma (B) starts in the mucosa; it would be unlikely to be biopsy negative. A lipoma (C) or a solitary fibrous tumor (E) would not likely erode into the mucosa. Melanoma (D) can metastasize to the stomach but does not typically present with a well-circumscribed mass.

\section{Answer D}

The patient is presenting with an unusual combination of an UGI bleed (coffee ground emesis) and obstructive jaundice (elevated bilirubin and rise in alkaline phosphatase with proportional rise in ALT and AST). Of the five options, ampullary cancer is the one that would most likely present as such. Pancreatic cancer $(\mathrm{C})$, though it certainly can cause pain, weight loss, as well as obstructive jaundice, is not associated with iron deficiency anemia or UGI bleed. Dieulafoy's lesion (E) typically presents with massive UGI bleeding, due to an abnormally located superficial arteriole that erodes. Diagnosis is difficult as it causes a pinpoint bleed that can only be seen when actively bleeding. Duodenal or gastric ulcers (A-B) would not cause obstructive jaundice.

\section{Answer B}

Massive hemoptysis with constitutional symptoms as described is likely from tuberculosis (TB). This is further supported by the patient's origin from a TB endemic area (e.g., Armenia, Mexico, Nigeria) and the CXR findings. The most common cause of massive hemoptysis in the world is TB. Immediate intubation is indicated to protect the airway. Once intubated and placed in the right lateral decubitus position (to prevent blood from entering the contralateral lung), the next best step in management involves localizing the source and controlling it with bronchoscopy. Emergent thoracotomy in the OR (A) can be considered if initial management options fail to control bleeding. VATS (C) offers a minimally invasive surgical technique to treat certain lung and chest wall diseases but is not currently used much in the emergency setting. Angiography (D) with embolization is a therapeutic option for massive hemoptysis. INH, rifampin, ethambutol, and pyrazinamide are used to treat TB but will not address the patient's acute condition.

\section{Answer A}

Melena can either be from an upper or a lower GI source. The presentation is classic for hereditary hemorrhagic telangiectasia (HHT) (also known as Osler-Weber-Rendu), an autosomal dominant disorder characterized by recurrent epistaxis, telangiectasia/red nodules of the face, lips, and/or GI tract, arteriovenous malformations (AVM), and a family history. GI bleeding most often occurs in the stomach and small bowel. If upper endoscopy is unable to confirm bleeding in the stomach, a capsule endoscopy (gelatin capsule containing a video recorder is swallowed, eventually retrieved in the stool, and permits visualization of the entire bowel) or push enteroscopy is used to assess for small bowel bleeding. Push enteroscopy uses a longer enteroscope or pediatric colonoscope to visualize the proximal jejunum. The subsequent step would be to perform lower endoscopy (C) (after a bowel preparation). However, colonic telangiectasias are very uncommon. Formal angiogram (D) would only be useful if the patient is actively bleeding at a rate of $1 \mathrm{ml} / \mathrm{min}$ (the patient is not currently bleeding), is an invasive procedure, and would not permit visualization of the extent of telangiectasias. Technetium-labeled red blood cell scan (E) may be useful if the patient is actively bleeding (at a rate of $0.5 \mathrm{ml} /$ min) but is particularly ineffective in localizing the site of bleeding in the small bowel. A CT angiogram (B) would not 
demonstrate telangiectasias of the small bowel (though it would be useful for identifying hepatic AVMs).

\section{Answer B}

The patient presented is suffering from a massive, ongoing UGI bleed. Management should always begin with the ABCs. Given the large amount of bleeding, combined with her hemodynamic instability, this patient's airway is at risk. As such, the correct answer is to emergently perform orotracheal intubation. Unlike an elective intubation, it should be performed using a rapid sequence intubation (RSI) technique. Reverse Trendelenburg (C) helps to prevent aspiration, but in the patient presented, it would be potentially dangerous given the marked hypotension. Following intubation and confirmation of appropriate placement, 2 liters of normal saline (D) would be administered. Given the massive bleeding, blood will also likely need to be administered. However, if the patient responds to the initial fluid bolus, O- blood may not be necessary (E). Following resuscitation, emergent endoscopy (A) to identify and treat the cause of the bleeding should be performed. Additionally, a Foley catheter should be placed to monitor urine output as an indication of the patient's volume status.

\section{Answer D}

The mainstay of therapy for UGI bleeding is endoscopic intervention. Techniques utilized include injection of epinephrine, sclerosing agents, clips, and cauterization. On rare occasion, if the bleeding cannot be controlled, then urgent surgical intervention is indicated. In the above case, the appropriate intervention is to surgically open the duodenum and oversew the ulcer in four quadrants to assure the artery has been ligated. Continuing blood transfusion alone is not appropriate (B). Although angiographic embolization (A) is occasionally utilized for GI bleeding in poor surgical risk patients, it would not be the first choice in someone who is hemodynamically unstable. Vasopressin (C) and octreotide (E) are used in the management of patients suffering from bleeding esophageal varices.

\section{Answer $\mathrm{E}$}

The case represents a classic history and endoscopic findings of a Mallory-Weiss tear. The etiology is thought to be a sudden and rapid rise in the transmural pressure gradient at the gastroesophageal (GE) junction, associated with retching. It is most often seen following an alcoholic binge but can also be seen with any forceful vomiting or coughing. It is thought to more likely occur in patients with hiatal hernias. The tear is longitudinal, partial thickness, in the stomach, near the GE junction. The left gastroduodenal artery (D) is not involved. It usually resolves spontaneously. Though it is seen following an alcohol binge, it is not related to portal hypertension (A), or H. pylori (C), and does not cause a pleural effusion (B). Mallory-Weiss is not to be confused with Boerhaave syndrome, which can also occur after forceful vomiting. In Boerhaave syndrome, the rapid rise in intrathoracic pressure can cause a full thickness perforation of the esophagus. The esophageal perforation results in esophageal contents spilling into the mediastinum and sometimes into the left chest, resulting in a left pleural effusion, subcutaneous emphysema, severe chest pain, and, if untreated, sepsis and death. Boerhaave syndrome requires broad-spectrum antibiotics and emergent surgery to seal the esophageal perforation and drain the mediastinal infection.

\section{Answer C}

GI bleeding, in association with a history of aortobifemoral bypass, is due to an aortoenteric fistula until proven otherwise. Patients typically have a "herald" bleed, followed later by exsanguinating hemorrhage (D). The NG aspirate returned clear fluid. This rules out bleeding from the stomach, but not from the duodenum. Thus the first diagnostic test of choice is still an upper endoscopy, but in aortoenteric fistulas, it is typically negative as the bleeding results from erosion of the aortic graft into the fourth portion of the duodenum which is usually not well visualized with a standard scope. The negative endoscopy essentially rules out the most common causes of UGI bleeding which include acute gastritis, as well as gastric and duodenal ulcers. A colonoscopy is generally not useful in the acute bleed setting, as the colon is filled with stool and clot. Thus, bowel prep is necessary. A negative lower endoscopy is less conclusive. Though it rules out colon cancer, other sources of lower GI bleed, such as diverticulosis and AVMs, may be hard to see if they are not actively bleeding. Nevertheless, with a negative upper and lower endoscopy, the bleeding is likely coming from the small bowel. This fulfills the definition of an obscure bleed (obvious bleeding, as evidenced by the melena, without an obvious source). An occult bleed is one that the patient is not aware of (detected only by fecal occult blood testing) (E). The diagnosis of an aortoenteric fistula is notoriously difficult to make. Angiography is poor at showing any abnormalities with an aortoenteric fistula (A). With an aortoenteric fistula, the aortic graft by definition is infected. Air, fluid, or stranding around the graft on CT scan beyond 6 weeks after surgery would be considered pathologic (B) and indicative of a graft infection. Treatment is to remove the graft. This can be done by either replacing it with a human cadaver homograft in situ or by performing an axillo-bifemoral bypass followed by removal of the aortic graft and aortic ligation.

\section{Answer $B$}

The most common cause of esophageal perforation is iatrogenic injury, usually from EGD, but also occasionally from endotracheal intubation or placement of a nasogastric tube. Boerhaave syndrome (A), foreign body (especially sharp or corrosive objects) (C), and esophageal cancer (D) may also 
cause perforation, but they are less common than iatrogenic injury. Trauma (E), especially of the penetrating variety, may injure the esophagus, but this is less common than iatrogenic injury.

\section{Answer D}

Boerhaave syndrome is the term used for a perforation of the esophagus that occurs following forceful emesis (iatrogenic perforation is not called Boerhaave syndrome) (B). The full thickness tear (C) causes spillage of GI contents into the mediastinum and even the thorax. Since GI contents spill into the mediastinum instead of the peritoneum, the patient does not develop peritonitis. Rather, they often present with fever and chest pain. As such, the diagnosis is often delayed until the patient becomes severely septic. Among GI perforations, it has the highest overall mortality. Boerhaave syndrome most often occurs in males between the ages of 50 and 70, after excessive intake of food and alcohol. Unlike Mallory-Weiss tears, Boerhaave syndrome does not typically present with UGI bleeding (A). Treatment is surgical (repair hole in esophagus) (E). Outcomes are dependent on the timeliness of recognition and surgical management.

\section{Answer D}

MALT lymphoma is strongly associated with $H$. pylori infection and is unique in that it is curable with antibiotics (i.e., triple therapy). In addition to biopsy, testing for $H$. pylori should be performed. After triple therapy, cure should be confirmed. If it is determined that triple therapy has failed, other modalities such as chemotherapy (A), radiation therapy (B), a combination of both $(\mathrm{C})$, and/or surgery (E) are utilized $(\mathrm{E})$.

\section{Answer E}

All patients presenting with possible esophageal perforation should receive immediate IV fluids and antibiotics for the treatment of sepsis. Gastric decompression (B), mediastinal and pleural drainage $(C)$, and primary repair (D) of the esophagus should occur after initial stabilization, ideally within 24 h. Esophagectomy (A) is reserved for extreme cases with large perforation or wherein primary repair will cause stricture.

\section{Answer B}

EUS is performed as it assists with TNM staging. EUS has been proven to provide more accurate assessment of tumor size, depth, and locoregional lymph node involvement compared to radiographic imaging. The next step is to perform a CT scan of the abdomen to look for distant metastasis and confirm that the patient is a surgical candidate. CT scanning will rule out liver metastasis as well as distant suspicious lymph nodes that were missed on EUS, either of which would preclude a curative resection. Though not the standard of care, PET scan (C) is emerging as an accurate modality for detecting small metastases and lymph node involvement. The management of gastric adenocarcinoma is dependent on the staging of the tumor. Surgery with gastric resection (A) is the only curative therapy to date for gastric cancer. Laparoscopy (D) is sometimes utilized to look for peritoneal or omental metastasis which would preclude curative gastrectomy. Gastric cancer that is more advanced than stage IB should be considered for a multi-therapeutic approach with chemotherapy (E).

\section{Answer B}

Eradication may be confirmed by a urea breath test, fecal antigen test (D), or upper endoscopy performed 4 weeks or more after completion of therapy. Typically, noninvasive methods should be performed except in cases when a followup endoscopy is indicated (e.g., gastric cancer, preneoplastic lesions, MALT lymphoma). Blood antibody test $(\mathrm{C})$ is unable to confirm eradication as $\operatorname{IgG}$ levels will remain elevated after the acute infection is resolved. Clinical resolution (E) of symptoms is inappropriate in confirming eradication of $H$. pylori, particularly in the setting of gastric cancer. 


\section{Urology}

\section{Areg Grigorian and Christian de Virgilio}

\section{Questions}

1. A 52-year-old male is brought to the hospital by his wife with complaints of intense pain that started around his right flank and now radiates to his right groin. He said that his urine appears pink. He appears to be in severe pain and is unable to remain still during examination. His abdominal exam is unremarkable. Urinalysis reveals $100 \mathrm{RBC} / \mathrm{hpf}$. IV fluids and analgesics are administered. Which of the following is the most appropriate imaging?
(A) Helical CT scan of the abdomen and pelvis without contrast
(B) Helical CT scan of the abdomen and pelvis with contrast
(C) Upright abdominal X-ray
(D) Intravenous pyelogram (IVP)
(E) Renal ultrasound

2. A 30-year-old male is involved in a high-speed MVC. He was wearing his seatbelt. In the ED, he has a strong odor of alcohol. Blood pressure is $120 / 70 \mathrm{mmHg}$ and heart rate is $80 / \mathrm{min}$. His abdomen appears distended and mildly tender diffusely. The pelvis is stable. On rectal exam, the prostate feels normal. There is no blood at the urethral meatus. On insertion of a Foley catheter, there is gross hematuria. CT scan of the abdomen and pelvis with oral and IV contrast reveals a large amount of free fluid and contrast in the peritoneum. The liver and spleen appear to be normal as does the pelvis. What part of the genitourinary tract is most likely injured?
(A) Ureter
(B) Base of the bladder
(C) Bladder dome
(D) Renal hilum
(E) Urethra

3. A 14-year-old boy is diagnosed with a varicocele by his family physician. Which of the following is true about this condition?
(A) It affects either side with similar frequency
(B) It causes the testicle to ride high in the scrotum
(C) It transilluminates on physical exam
(D) It is related to impaired venous drainage
(E) It is associated with an absent cremasteric reflex

4. A 32-year-old newlywed man presents to the ED with intense pain in his penis. He reports having an accident falling in the bathroom. His temperature is $98.8^{\circ} \mathrm{F}$, blood pressure is $126 / 80 \mathrm{mmHg}$, and pulse is $110 / \mathrm{min}$.
His physical examination is significant for a blue discoloration at the base of a swollen and deviated penis. He has no ulcers or discharge. Retrograde urethrogram did not identify any urethral injuries. What is the best next step for this patient?
(A) Abdominal and pelvic CT scan
(B) Cystoscopy
(C) Analgesics and bed rest
(D) Surgical repair
(E) Temporary suprapubic catheter

5. A 78-year-old man arrives to the ED with colicky flank pain for the past 4 days that is now accompanied by nausea, vomiting, fever, and hematuria. Past medical history is significant for congestive heart failure and prior myocardial infarction. On physical examination, the patient has a blood pressure of $100 / 60 \mathrm{mmHg}$, temperature of $101{ }^{\circ} \mathrm{F}$, and a heart rate of $110 / \mathrm{min}$. Urinalysis reveals $150 \mathrm{RBC} / \mathrm{hpf}$ and $20 \mathrm{WBC} / \mathrm{hpf}$. Laboratory tests demonstrate a WBC of $15 \times 10^{3} / \mu \mathrm{L}$ (normal $4.1-10.9 \times 10^{3} / \mu \mathrm{L}$ ) with $10 \%$ bands. Imaging demonstrates a 10-millimeter stone lodged in the ureterovesicle junction with dilation of the right renal calyx. Broad-spectrum antibiotics are administered intravenously. What is the best next step in management?
(A) Percutaneous nephrostomy tube
(B) Open nephrostomy
(C) Shock wave lithotripsy
(D) Placement of a ureteral stent
(E) Admit to ICU for close monitoring

6. Which of the following is true regarding the circulation to the testes?

(A) Each testis has a singular blood supply, which arises from the aorta

(B) Testicular ischemia after inguinal hernia repair is most often due to accidental transection of the testicular artery

(C) An ischemic testis does not lead to antisperm antibody formation

(D) During surgery for an undescended testis, the testicular artery may need to be divided

(E) Lymphatic drainage from the testis is to the inguinal lymph nodes

7. A 45-year-old male presents with a new scrotal mass on the right side that he noticed a week earlier. It is nontender. He reports no history of trauma. When the patient is standing, the scrotal mass is separate from the testicle and epididymis, feels like a "bag of worms," and is soft, compressible, and non-tender. With the patient supine, the mass is unchanged. The left side is normal. What is the most appropriate next step in the management? 

(A) Scrotal support and NSAIDs
(B) Surgical intervention
(C) CT of the abdomen
(D) Reassurance and observation
(E) Ultrasound-guided biopsy of the mass

8. A 15 -year-old boy arrives to the ED $4 \mathrm{~h}$ after experiencing a sudden onset of right testicular pain while playing soccer. He does not recall any specific trauma to his testicle during the game. He also reports nausea and vomiting. Physical examination reveals a tender and swollen right testicle that is displaced superiorly. The testicle appears to be lying transversely. He has an absent cremasteric reflex on the right. The left testicle is normal in location and is non-tender. What is the next step in management?
(A) Ultrasound
(B) Color Doppler ultrasound
(C) Right orchiopexy
(D) Bilateral orchiopexy
(E) Scrotal support and nonsteroidal anti-inflammatory drugs

9. A 37-year-old obese woman arrives to the ED with left flank pain and hematuria. She has never experienced these symptoms before. Her past medical history includes Crohn's disease which has been controlled with mesalamine. She is afebrile with a blood pressure of $130 / 84 \mathrm{mmHg}$ and a pulse of 104/min. Physical examination reveals a laparotomy scar in her right lower quadrant. She is given analgesics for pain control. What is the most likely etiology of her acute symptoms?
(A) Gallstones
(B) Hypercalciuria
(C) Increased absorption of oxalate
(D) Protease producing bacteria
(E) Mesalamine

10. A 10-year-old boy presents to his doctor for follow-up 2 weeks after having an upper respiratory tract infection (URI). He complains of pain in his scrotum. It has been bothering him for the past 3 days but is more painful today. A scrotal ultrasound demonstrates an enlarged and rounded epididymis. His scrotal skin appears thickened. What other finding(s) would you suspect in this patient's history and physical?
(A) Skin lesions and abdominal pain
(B) Recent weight loss, night sweats, and fevers
(C) Recent epididymitis
(D) Sores in the mouth and swelling of the eyes
(E) Painful urination

11. An infant is found to have an undescended left testicle shortly after birth. During his 6-month checkup, the left testicle remains undescended. What can be done to decrease this patient's risk of developing testicular cancer later in life to match that of a boy born without cryptorchidism?

(A) Immediate orchiopexy

(B) Orchiopexy within the next 6 months

(C) Hormonal therapy

(D) Observation until age two and then orchiopexy

(E) Risk cannot be decreased to that of a boy without cryptorchidism

12. A 26-year-old man that recently emigrated from Sudan to the United States arrives to your office complaining of red urine. He does not experience any pain but says that for the last week his urine has appeared red. He also reports night sweats, fevers, and losing 10 pounds over the last 2 months without a change in his appetite. Cystoscopy is performed, and biopsies are taken of a tumor that appears nodular with a plaque-like and irregular surface. What is most likely to be seen on microscopic analysis?

(A) Urothelial carcinoma (transitional cell carcinoma)

(B) Squamous cell carcinoma

(C) Adenocarcinoma

(D) Mucinous (colloid) carcinoma

(E) Small cell carcinoma

13. A 7-year-old boy presents to the doctor with a non-tender scrotal mass. His vitals are normal and stable and he has no other complaints. His mother reports that he has been behaving more aggressively at home and has gotten into two fights at school over the past month. Physical examination reveals a firm $2.5-\mathrm{cm}$ mass within the right testicle. There are no epididymal masses bilaterally, although both testicles appear to be enlarged. He also has axillary hair. Laboratory analysis reveals a normal urinalysis. What is the most likely etiology of the testicular mass?
(A) Leydig cell tumor
(B) Sertoli cell tumor
(C) Seminoma
(D) Teratoma
(E) Juvenile granulosa cell tumor

14. A male infant presents with a mass in his scrotum that has been present since birth. His mother states that the mass changes in size when the infant cries and does not seem to be painful. On exam, the mass appears to be adjacent to his testicle which is palpated posteriorly. The mass is soft and non-tender and transilluminates. There is no bulge or mass at the internal ring. What is the most appropriate next step in the management?

(A) Surgical management
(B) Medical management 

(C) Ultrasound
(D) Needle aspiration
(E) Reassurance

15. A 50-year-old man arrives to the ED with severe acute colicky flank pain and hematuria. The patient has a long-standing history of gout. Which of the following is true regarding the type of kidney stone the patient likely has?
(A) Most are radiopaque
(B) They are often seen in patients with hyperparathyroidism
(C) Shock wave lithotripsy is not helpful
(D) Sodium bicarbonate administration is beneficial
(E) Suppressive antibiotics are helpful in prevention

16. A 20-year-old male presents with a right testicular mass. He noticed the mass after getting kneed in the groin during a basketball game 3 weeks earlier. He states that the mass is not tender. He denies any other symptoms. On physical examination, there is a firm 2-cm mass within the right testicle that is not tender. Ultrasound confirms a 2-cm homogeneous mass within the right testicle. CT scan of the abdomen and pelvis is negative. CXR is negative. Which of the following is the most appropriate next step in management?
(A) Radical inguinal orchiectomy
(B) Scrotal orchiectomy
(C) Fine needle aspiration
(D) Open testicular biopsy
(E) Percutaneous biopsy 


\section{Answers}

\section{Answer A}

The presentation is consistent with nephrolithiasis. Initial management should focus on IV fluid hydration and analgesia. Recommended imaging includes a KUB (a supine X-ray of the abdomen) and a non-contrast $\mathrm{CT}$ of the abdomen and pelvis. The use of contrast (B) may interfere with visualization of the stone. An upright abdominal X-ray (C) is used to look for air-fluid levels in association with a small bowel obstruction or free air under the diaphragm. Such a film cuts off the pelvis and as such will miss many ureteral stones. IVP (D) has largely been replaced by CT. Renal ultrasound (E) may be used in pregnant patients but may miss stones; it is also used to look for hydronephrosis as an adjunct to KUB (if a CT scan is not obtained).

\section{Answer $\mathrm{C}$}

Injuries to the genitourinary tract are commonly seen following blunt trauma. Bladder injuries typically occur when the bladder is full (such as following a drinking binge). Injuries to the bladder are divided into intraperitoneal and extraperitoneal. Intraperitoneal injuries will demonstrate a large amount of intraperitoneal free fluid (and contrast) on CT scan. Although the free fluid might initially be thought of as being blood, the absence of liver or spleen injury raises suspicion that the fluid is urine. Intraperitoneal injuries require laparotomy and repair. The bladder dome is the only region covered by the peritoneum and is considered the weakest portion; thus, it is prone to rupture. However, the most common overall location of bladder rupture is at the base of the bladder (B), which is located in the retroperitoneum. Retroperitoneal bladder injuries are most often associated with pelvic fractures and can be managed nonoperatively. The ureter (A) and renal hilum (D) are also located in the retroperitoneum. Urethral injury $(\mathrm{E})$ is associated with blood at the urethral meatus and would not be expected to cause free fluid in the peritoneum.

\section{Answer D}

Varicocele is often an asymptomatic condition, but patients may complain of a vague discomfort and/or pain in the scrotum. The affected side will have a mass that feels similar to a "bag of worms" and will disappear upon lying down and reappear when the patient stands up. Varicoceles develop as a result of tortuous dilation of the pampiniform plexus of the veins surrounding the spermatic cord and testis. About $80 \%$ occur on the left side (A). Since it is a venous drainage problem, varicoceles increase in size with Valsalva. Unlike a hydrocele that transilluminates (as it contains clear fluid), a varicocele does not $(\mathrm{C})$. A testicle that is high in the scrotum (B) would raise concern for testicular torsion (if associated with sudden pain) or a partially undescended testicle. An absent cremasteric reflex (E) is seen with testicular torsion.

\section{Answer D}

The history of sudden penile pain following trauma combined with the physical examination is consistent with a penile fracture, which requires urgent surgical repair. It is due to a tear in the tunica albuginea with subsequent rupture of one or both of the corpus cavernosum. Failure to recognize and surgically manage the injury often results in permanent erectile dysfunction. Men are often embarrassed to describe the true nature of the injury so they fabricate stories that don't fit the clinical picture. Penile fractures most often occur during vigorous sexual activity. Most patients describe a "snapping" sound preceding the fracture. A hematoma rapidly develops within the corpus cavernosum and presents as a blue discoloration at the base of the penis, which is deviated at the fracture site. Prior to surgical exploration, a urethral injury should be ruled out with a retrograde urethrogram. CT scan (A) and cystoscopy (B) play no role in the evaluation of penile fractures. Analgesics and best rest (C) are not acceptable options for a patient suspected of having a penile fracture. Temporary suprapubic catheter (E) would not help address the underlying problem.

\section{Answer A}

Ureteral obstruction in association with sepsis requires emergent urinary decompression. This is most expeditiously achieved via a percutaneous nephrostomy tube. Shock wave lithotripsy (C) is unlikely to relieve the obstruction caused by a stone of this size. Open nephrostomy (B) is rarely indicated. Close monitoring in the ICU (E) as the sole management plan would be inappropriate for a patient with sepsis secondary to a blocked ureter. Hydration, analgesics, and bed rest would be appropriate for an uncomplicated and small renal stone without accompanying hydronephrosis. A ureteral stent (D) is an option; however, it is a more time-consuming procedure that will not be as expeditious in a septic patient compared to a percutaneous nephrostomy.

\section{Answer D}

The testes receive blood from three (A) sources: a testicular artery that arises directly from the aorta, from the cremaster artery which is a branch of the inferior epigastric artery, and from the deferential artery, or the artery to the ductus deferens which is a branch of the superior vesical artery. As such, the testicular artery can be divided in most patients without testicular ischemia developing. In fact, the testicular artery is sometimes intentionally divided in the setting of an undescended testicle, when the testicle is very high in the retroperitoneum and additional length is needed to bring it into the scrotum. The most common cause of testicular ischemia after inguinal hernia repair is injury to the pampiniform plexus which causes severe testicular congestion and subsequent ischemia (B). A blood-testis barrier exists, presumably to prevent sperm (which are formed during adolescence) 
from coming in contact with the blood. In the setting of testicular necrosis, antisperm antibodies can form and adversely affect future fertility (C). Lymphatic drainage from the testicle is to periaortic nodes $(\mathrm{E})$. This needs to be recognized in the metastatic work-up of testicular cancer.

\section{Answer C}

A scrotal mass that feels like a "bag of worms" is the classic description for a varicocele, which essentially represents dilated veins in the pampiniform plexus. Sudden onset of a right-sided varicocele should raise suspicion for a renal cell carcinoma that has occluded the inferior vena cava (IVC). The vast majority ( $80 \%$ ) of varicoceles are left sided due to the higher likelihood of impaired drainage of the left testicular vein (it enters the left renal vein at a right angle). Drainage of the right testicular vein is better as it enters at a less acute angle and directly into the inferior vena cava (IVC). Thus sudden onset of a right-sided varicocele raises suspicion that the IVC is obstructed. The best initial test is to perform a CT scan of the abdomen to look for a renal or other retroperitoneal mass. There is no known effective medical management for varicoceles, so that scrotal support and NSAIDs (A) would not be recommended. Varicoceles are associated with infertility (due to low sperm count and decreased motility). Surgical intervention (B) is considered for such an indication. Reassurance and observation (D) would not be appropriate as further work-up is indicated. Since a varicocele is a nest of dilated veins, attempts to biopsy would be contraindicated $(\mathrm{E})$.

\section{Answer D}

The history and physical are classic for testicular torsion, including the age (adolescent), sudden onset of pain, testicular swelling, superior displacement of the testicle, and an absent cremasteric reflex. Constitutional symptoms (nausea and vomiting) are also common. Testicular torsion represents an emergency, as time is of essence. If a testicular torsion is reduced within $6 \mathrm{~h}$ of onset, there is a very high testicular salvage rate ( $>90 \%$ ), whereas with delays in management beyond $24 \mathrm{~h}$, the testicular salvage rate plummets to $<10 \%$. If the diagnosis is in doubt, color duplex scan (ultrasound with Doppler) (B) is recommended to look for the absence of blood flow in the affected testicle, but would be inappropriate in a patient with a clear-cut presentation as it would delay management. Ultrasound (A) is not helpful as it does not provide blood flow information. Treatment is surgical, via orchiopexy. Since the contralateral testicle is at risk, bilateral orchiopexy is recommended (C). Scrotal support and NSAIDs (E) would be inappropriate.

\section{Answer C}

Patients with Crohn's disease that present with flank pain and hematuria should raise suspicion for nephrolithiasis secondary to hyperoxaluria. Her laparotomy scar suggests that she had an ileocolic resection, which would predispose her to fat malabsorption as the terminal ileum is the principal site for fat absorption. In healthy patients, calcium binds to oxalate to prevent its absorption from the GI tract. In patients with increased amounts of fat in the GI lumen (e.g., Crohn's statuspost ileocolic resection), the calcium preferentially binds to fat leaving the unbound oxalate available for reabsorption and, thus, increases the risk of developing calcium oxalate renal stones. Hypercalciuria (B) would have been the most likely etiology had she not had Crohn's. Protease producing bacteria (D) are associated with struvite stones and recurrent urinary tract infections. Gallstones (A) do not cause flank pain or hematuria, and mesalamine $(\mathrm{E})$ is not a known risk factor for the development of renal stones.

\section{Answer A}

This patient's history and ultrasound findings are suggestive of Henoch-Schönlein purpura (HSP). This classically develops after an URI or drug exposure (e.g., vancomycin) in young children. HSP is typically characterized by nonthrombocytopenic purpura, arthralgia, abdominal pain, intussusception, and, less frequently, scrotal pain. Rarely, scrotal pain may be the only manifestation of HSP. The sonographic findings of an enlarged, rounded epididymis are sufficiently characteristic to allow distinction from torsion in most cases. HSP patients may also develop thickened scrotal skin. Recent weight loss, night sweats, and fevers (B) can represent the constitutional symptoms seen in patients with testicular cancer. However, these patients present with a painless testicular mass. A young boy with no history of sexual activity would not be expected to have epididymitis (C) or a urethral tract infection with painful urination $(\mathrm{E})$. Sores in the mouth and swelling of the eyes (D) are seen in patients with Behcet's syndrome, a form of vasculitis. Patients with Behcet's can also develop scrotal pain secondary to open sores. However, Bechet's is very rare in the United States and more commonly seen in the Middle East and Asia.

\section{Answer E}

Infants born with cryptorchidism have an increased risk of developing testicular cancer later in life. An undescended testicle may be palpable high up in the inguinal canal, or nonpalpable, in which case it may be in the retroperitoneum or absent. Orchiopexy is a procedure to move an undescended testicle into the scrotum and anchor it to the scrotal wall. Orchiopexy has several theoretical benefits. It may improve fertility. An undescended testicle is thought to the increase risk of infertility, as the warmer environment of the retroperitoneum or the inguinal canal leads to impairment of germ cell maturation. Undescended testicles are at higher risk of torsion. Orchiopexy prevents such a catastrophic event. Orchiopexy permits regular physical examination so as to detect any potential malignancies later in life. However, the act of lowering the testicle does not lower its future risk 
of developing a malignancy (thus answers A-D are incorrect). The higher the testicle is found away from the scrotum, the higher the likelihood of developing testicular cancer later in life. Although the precise time to perform orchiopexy is not well established, most clinicians elect to do it shortly after the patient's first year of life so as to reduce the risk of infertility. Hormonal therapy (C) with intramuscular beta-hCG injection is sometimes used in an attempt to induce testicular descent; however, it is successful in only a minority of patients.

\section{Answer $B$}

The most common type of bladder cancer in the United States is urothelial carcinoma (transitional cell carcinoma) (A) and is well known to be associated with environmental carcinogens such as smoking and polycyclic aromatic hydrocarbons. However, in parts of the world that are endemic for schistosomiasis, such as Sudan, Egypt, and Tanzania, the most common variant is squamous cell carcinoma. The chronic granulomatous cystitis secondary to the parasite can result in squamous metaplasia and subsequent development of squamous cell carcinoma. Adenocarcinoma of the bladder (C) is rare in the developed world. Mucinoid carcinoma (D) is a subtype of adenocarcinoma and is characterized by abundant extracellular mucin. Small cell carcinoma (E) is often considered a subtype of urothelial carcinoma that is accompanied by small cell differentiation.

\section{Answer A}

Leydig cell tumors are benign sex cord-stromal tumors that are associated with high levels of androgen production that may result in precocious puberty in young boys. These children can behave more aggressively and develop early secondary sexual characteristic changes such as enlarged testicles, scrotum, and/or penis, and accelerated linear growth. Characteristic rod-shaped Reinke crystals may be seen on histology for Leydig cell tumors. Scrotal ultrasound would be the next diagnostic step as this can help visualize the mass in question and is also important to help evaluate the contralateral testicle. Ultrasound might also help distinguish between benign lesions, which are more often hyperechoic, and solid lesions that are more likely to be malignant and appear hypoechoic. Sertoli cell tumors (B) are benign and often clinically silent. Seminoma, a germ cell tumor, is the most common type and is considered malignant $(C)$. Teratomas (D) can be benign or malignant, are derived from $\geq 2$ embryonic layers, and are associated with AFP or hCG production. Granulosa cell tumor $(\mathrm{E})$ is far more common in females and thus is associated most frequently with ovarian cancer.

\section{Answer $E$}

A hydrocele is a fluid collection within the processus and/or tunica vaginalis of the scrotum. Communicating hydroceles develop as a result of failure of the processus vaginalis to close during embryologic development, allowing for peritoneal fluid to enter the scrotum. Noncommunicating hydroceles have no connection to the peritoneum, and fluid accumulation develops from the mesothelial lining of the tunica vaginalis. The majority of infants born with hydroceles will have spontaneous resolution by the time they are 1 year old; thus reassurance and observation are the most appropriate forms of management. Surgical repair (A) is not typically done unless the hydrocele is persistent past the first year of life. There are no available medical therapies (B) for managing hydroceles. There is no indication for ultrasound (C) with this classic presentation. Hydroceles are sometimes confused with hernias; however, ultrasound is neither sensitive nor specific for distinguishing the two. Ultrasound would be more suitable for ruling out malignancy in a young adult with a testicular mass. There is no role for needle aspiration (D).

\section{Answer D}

The most likely diagnosis in a patient with a past medical history significant for gout presenting with acute colicky pain and hematuria is nephrolithiasis secondary to uric acid renal stones. Unlike other types of renal stones, this type is radiolucent (A) and will not show up on X-ray. Patients with gout are at increased risk for developing uric acid stones. Sodium bicarbonate will alkalinize the urine to achieve a urinary $\mathrm{pH}$ of 6-6.5, as this would provide optimal conditions for dissolution of uric acid stones. Patients with hyperparathyroidism are more prone to developing calcium oxalate renal stones (B). Suppressive antibiotics (E) should be considered in the case of struvite stones secondary to recurrent urinary tract infections. Shock wave lithotripsy (C) may be added as an adjunct to urine alkalinization to further improve the stonefree rate.

\section{Answer A}

The demonstration on ultrasound of a solid mass (e.g., homogeneous) within the testicle makes the likelihood of cancer very high. Any painless mass within the testicle is cancer until proven otherwise. Most patients with testicular cancer present without symptoms and most are young adults (average age between 20 and 35 years). Trauma to the scrotum or groin may prompt men to examine their testes leading to the discovery of an otherwise painless mass. In patients with a testicular mass that is highly suspicious for malignancy (based on physical exam and ultrasound), radical inguinal orchiectomy is performed. Orchiectomy via a trans-scrotal incision (B) is associated with a higher rate of local recurrence. The inguinal incision also allows a longer portion of the spermatic cord to be removed. There is no role for biopsy (C-E) as there is a high risk of seeding or spreading the cancer with biopsy. 


\section{Vascular}

\section{Christian de Virgilio, Areg Grigorian, and Paul N. Frank}

\section{Questions}

1. A 67-year-old male smoker with a known abdominal aortic aneurysm (AAA) comes to the ED with the acute onset of flank pain. On physical exam, his blood pressure is $71 / 49 \mathrm{mmHg}$, pulse is $121 / \mathrm{min}$, temperature is $98.9^{\circ} \mathrm{F}$, and respirations are $22 / \mathrm{min}$. His abdominal exam reveals distention, a pulsatile abdominal mass, and diffuse tenderness without rebound. What is the most appropriate next step in management?
(A) CT scan of the abdomen
(B) Exploratory laparotomy
(C) Transfusion of packed RBCs
(D) Abdominal ultrasound
(E) Diagnostic peritoneal lavage

2. A 56-year-old man is seen in the ED for onset of severe pain and numbness in his left leg that began 30 min ago. He has a past medical history significant for hypertension, diabetes, and hyperlipidemia. Physical exam reveals a temperature of $98.3{ }^{\circ} \mathrm{F}$, blood pressure of $134 / 74 \mathrm{mmHg}$, pulse of $89 / \mathrm{min}$, and respiratory rate of $16 /$ min. Heart is irregularly irregular with no murmurs appreciated. On extremity exam, there is absent pulses in the left femoral, popliteal, and pedal arteries. On the right, all pulses are $2+$. The left foot is cool to touch as compared to the right. Sensation to pinprick is decreased on the left dorsum of the foot compared to the right. What is the most likely etiology of these findings?
(A) Arterial thrombosis
(B) Peripheral neuropathy
(C) Arterial embolism
(D) Cerebrovascular accident
(E) Venous thrombosis

3. A 68-year-old male with a 50-pack-year history of smoking presents with $2 \mathrm{~h}$ of left arm weakness. Physical exam shows $1+$ strength in the left upper extremity and $3+$ strength in the proximal left lower extremity. Which of the following is the next step?

(A) Doppler ultrasound of the right carotid artery

(B) Echocardiogram

(C) Non-contrast CT brain

(D) ECG

(E) CT angiogram of the neck

4. A 59-year-old male comes to see his primary care physician endorsing a 10-month history of increasing bilateral calf pain with exercise. He initially only felt discomfort after walking six blocks but now it occurs after only one block. The pain disappears with rest. His past medical history is significant for hypertension and hypercholesterolemia, for which he takes lisinopril and a statin. He has smoked two packs per day for the past 40 years. His physician orders a complete work-up and decides the patient most likely is suffering from claudication secondary to peripheral arterial disease (PAD). Which of the following findings would be the best way to support the diagnosis of PAD?

(A) History of pain in legs with exertion

(B) Diminished peripheral pulses on physical examination

(C) A combination of history and physical examination

(D) An ankle-brachial index $<0.9$

(E) Arteriographic evidence of atherosclerotic plaques

5. A 65-year-old male comes to the physician for a routine physical exam. He has a past medical history significant for hypertension and hyperlipidemia. He is on hydrochlorothiazide, aspirin, and a statin. He has a 10-packyear smoking history but quit over 30 years ago. Which of the following screening tests is appropriate for this male?

(A) CT scan of the abdomen

(B) Duplex scan of the carotid arteries

(C) Abdominal ultrasonography

(D) Pulmonary spirometry

(E) Ankle-brachial index (ABI)

6. A 52-year-old male is seen at the ED for the acute onset of swelling in his right leg that began $6 \mathrm{~h}$ ago. He has a past medical history significant for hypertension and colon cancer being treated with chemotherapy in the outpatient setting. Physical exam reveals a temperature of $99.1^{\circ} \mathrm{F}$, blood pressure of $125 / 72 \mathrm{mmHg}$, pulse of $77 /$ $\mathrm{min}$, and respiratory rate of $16 / \mathrm{min}$. On extremity exam, there are $2+$ posterior tibialis and dorsalis pedis pulses bilaterally. The right leg is edematous, warm, and markedly more swollen than the left leg. What is the best initial study in the work-up?
(A) Ankle-brachial index (ABI)
(B) Venous duplex scan
(C) CT angiogram of lower extremities
(D) CT of the head
(E) Measurement of compartment pressures

7. Which of the following patients should undergo carotid endarterectomy (CEA)?

(A) 70-year-old male with a history of transient painless right eye vision loss and $75 \%$ stenosis of the right internal carotid artery (ICA) 
(B) 70-year-old male with a history of transient painless right eye loss and $100 \%$ occlusion of the right ICA

(C) 65-year-old female who sustained a stroke 1 week ago, is aphasic, has no motor function in her right arm or leg, and has $80 \%$ left ICA stenosis

(D) 68-year-old male who, 4 days ago, became acutely dizzy and passed out with $60 \%$ right ICA

(E) 72-year-old male with an irregularly irregular rhythm who presents with left-sided weakness for the last several hours and has a $40 \%$ right ICA stenosis

8. A 70-year-old male presents with right calf pain after walking three blocks, forcing him to stop and rest. He denies pain at rest in his foot. He smokes one pack per day. His pulse is $74 / \mathrm{min}$, respiratory rate is $16 / \mathrm{min}$, and blood pressure is $136 / 86 \mathrm{mmHg}$. Physical examination reveals diminished pulses in his right foot. The anklebrachial index on the right leg is 0.7 . The next step in the management is:
(A) Smoking cessation
(B) CT angiogram
(C) MR angiogram
(D) Formal angiogram
(E) Stenting of iliac artery

9. A 55-year-old male comes to the physician complaining of bilateral leg pain with walking that is relieved by rest. $\mathrm{He}$ has a history of hypertension, hyperlipidemia, chronic obstructive pulmonary disease, and a 30-packyear smoking history. On physical exam, his blood pressure is $139 / 79 \mathrm{mmHg}$, temperature is $99.3^{\circ} \mathrm{F}$, pulse is $89 / \mathrm{min}$, and respirations are $16 / \mathrm{min}$. Present behind both knees are small pulsatile masses. What is most strongly associated with this finding?
(A) Aortic dissection
(B) Hypercoagulable state
(C) Degenerative joint disease (DJD)
(D) Abdominal aortic aneurysm (AAA)
(E) Thoracic aortic aneurysm

10. A 62-year-old man presents to the physician with swelling in both legs that has been going on for several months. He has a past medical history significant for hypertension and hyperlipidemia. He states that the swelling becomes progressively worse throughout the day. He denies any pain associated with the swelling. Physical exam reveals a temperature of $99.1^{\circ} \mathrm{F}$, blood pressure of $125 / 72 \mathrm{mmHg}$, pulse of $77 / \mathrm{min}$, and respiratory rate of $16 / \mathrm{min}$. Heart is in regular rate and rhythm with no murmurs. JVP is $7 \mathrm{~cm}$. The lungs are clear to auscultation. On extremity exam, there are strong posterior tibialis and dorsalis pedis pulses bilaterally. The legs are edematous up to the knee with a small patch of eczematous, erythematous-brown scaling skin rash located on the medial malleolus of the right ankle. Which of the following is the most likely cause of his edema?
(A) Peripheral artery disease
(B) Deep venous thrombosis
(C) Renal protein loss
(D) Reduced cardiac output
(E) Valve incompetence

11. At a follow-up appointment 2 weeks after undergoing left carotid endarterectomy (CEA), it is observed that the patient's tongue deviates to the left when he is asked to stick his tongue out. Which of the following is the most likely explanation?

(A) Perioperative stroke of the medulla

(B) Hematoma compressing the musculature of the oropharynx

(C) Injury to cranial nerve VII

(D) Injury of cranial nerve $\mathrm{X}$

(E) Injury of cranial nerve XII

12. A 61-year-old man comes to the physician with an 8-month history of increasing bilateral calf pain during his morning walks. The pain disappears with rest. He reports that the last time he saw a doctor was 20 years ago. He has a 30 pack year smoking history, but recently quit. His pulse is $68 / \mathrm{min}$, respirations are $18 / \mathrm{min}$, and blood pressure is $126 / 76 \mathrm{mmHg}$. His total cholesterol is $320 \mathrm{mg} / \mathrm{dL}$. His doctor recommends that the patient starts a statin and aspirin to reduce the risk of adverse cardiac events. In addition, he recommends that the patient begins an exercise walking program. What would the addition of this intervention provide for the patient?

(A) It will improve his overall cardiac health but will not improve his walking distance

(B) It would be expected to result in an increase in his ankle-brachial index

(C) It is less effective than administering a vasodilator

(D) It can result in a doubling of his walking distance

(E) It is contraindicated

13. A 75-year-old male is recovering in the ICU from an open repair of a ruptured abdominal aortic aneurysm. On post-op day 1, he complains of abdominal pain and tenderness over the left lower quadrant, without rebound or guarding. On post-op day 2 , he develops bloody diarrhea. Physical exam reveals a blood pressure of $129 / 79 \mathrm{mmHg}$, pulse of $100 / \mathrm{min}$, temperature of $99.9^{\circ} \mathrm{F}$, and respirations of $16 / \mathrm{min}$. What is the most likely etiology of his abdominal pain and bloody diarrhea? 

(A) Colonic ischemia
(B) Clostridium difficile colitis
(C) Diverticulitis
(D) Inflammatory bowel disease
(E) Colon cancer

14. A 44-year-old female is brought to the ED after a vehicle sideswiped her while she was on her motorcycle. She is complaining of right leg and knee pain. Initial survey reveals stable airway, breathing, and circulation. On exam, her heart has regular rate and rhythm; lungs are clear. No abdominal tenderness of rebound. There is $0 / 5$ motor strength in dorsiflexion of the foot and decreased sensation to pinprick at the dorsum of the foot. The extremity is warm and well perfused, and dorsalis pedis, posterior tibialis, and popliteal pulses are $2+$ bilaterally. What is the most likely etiology of her neurologic deficits?
(A) Common peroneal nerve injury
(B) Compartment syndrome
(C) Arterial thrombosis
(D) Deep peroneal nerve injury
(E) Pain from the fracture

15. A 74-year-old male presents to the ED with loss of vision in his right eye. Which of the following findings is most suggestive of a nonsurgical diagnosis?

(A) Bruit on auscultation of the right carotid artery

(B) Jaw pain with chewing

(C) Eighty percent stenosis of the right carotid artery on CT angiogram

(D) Left hand weakness

(E) Atrial mass on echocardiogram

16. In a patient with claudication, the 5-year risk of limb loss is about:
(A) $2-5 \%$
(B) $8-10 \%$
(C) $20-25 \%$
(D) $40-50 \%$
(E) $70-80 \%$

17. A 69-year-old immigrant from Mexico comes into the hospital with a 2-day history of severe pain in his left calf. He took Vicodin that a neighbor gave him with only minimal relief. Over the last day, he has lost sensation and movement in his left foot, which prompted him to finally come to the hospital. He has had no medical care and has no past medical history. Physical exam reveals a temperature of $98.5^{\circ} \mathrm{F}$, blood pressure of $147 / 79 \mathrm{mmHg}$, heart rate of $85 / \mathrm{min}$, and respiratory rate of $16 / \mathrm{min}$. On extremity exam, there are absent popliteal, posterior tibialis, and dorsalis pedis pulses on the left. Sensation to pinprick is absent in the left, and he cannot plantar- or dorsiflex his foot. The skin of the foot appears to be mottled. Doppler of the foot vessels reveals no audible signals. What is the definitive treatment?
(A) Catheter-assisted thrombolysis
(B) Open surgical intervention
(C) Amputation
(D) Aspirin
(E) Intravenous heparin

18. A 71-year-old man comes in to the physician, along with his wife, and reports a 4-month history of increasing left calf pain with exercise. He works as a grocery store manager and describes having the pain every morning after he walks from his car in the parking lot to the grocery store. The pain disappears once he is able to sit down. He has smoked one pack per day for the past 50 years. His pulse is $88 / \mathrm{min}$, respirations are $18 / \mathrm{min}$, and blood pressure is $156 / 96 \mathrm{mmHg}$. His total cholesterol is $300 \mathrm{mg} / \mathrm{dL}$. His right ABI is 0.7 . His wife is very worried and asks the physician what his prognosis is. In patients with this condition, the 5-year survival is:
(A) The same as that of age- and gender-matched controls without claudication
(B) Reduced primarily due to the risk of limb gangrene
(C) Reduced primarily due to lung cancer
(D) Reduced primarily due to coronary artery disease
(E) Reduced primarily due to stroke

19. A 61-year-old patient with coronary artery disease, diabetes, and hypertension presents to the ED with difficulty speaking and right-sided hemiparesis. His wife reports that he had multiple episodes a few days ago where he had difficulty speaking but they only lasted a few minutes. A CT scan of the head without contrast did not identify any hemorrhage in the brain. His systolic blood pressure is found to be $230 \mathrm{mmHg}$. What is the most appropriate next step in management?
(A) Labetalol
(B) Nimodipine
(C) Nitroglycerin
(D) Nitroprusside
(E) Hydralazine

20. A 62-year-old male presents to the physician with pain in his buttocks that comes on during his evening walks with his wife, forcing him to stop and rest. He also confides in you that he has been having difficulty maintaining an erection leading to marital problems. Physical exam is significant for absent femoral and distal pulses. What is the most likely diagnosis? 

(A) Leriche syndrome
(B) Acute occlusion of the infrarenal aorta
(C) Extensive atherosclerosis of the superficial femoral artery
(D) Spinal stenosis
(E) Bilateral hip osteoarthritis

21. A 65-year-old male presents with new onset claudication. An ABI confirms that he has peripheral arterial disease (PAD). Which of the following would provide the least amount of benefit to the patient?
(A) Warfarin
(B) Aspirin
(C) Statin
(D) Cilostazol
(E) Clopidogrel

22. A 30-year-old male presents with a 4-month history of a painful right great toe. In addition, he states that when he walks about two blocks, his right foot hurts and he has to stop to rest. He has a 20 pack year smoking history. His pulse is $74 / \mathrm{min}$, respirations are $18 / \mathrm{min}$, and blood pressure is $126 / 80 \mathrm{mmHg}$. His total cholesterol is $160 \mathrm{mg} / \mathrm{dl}$. His serum calcium is $9.4 \mathrm{mg} / \mathrm{dL}$, and on physical examination, he has normal femoral and popliteal pulses bilaterally, but no palpable dorsalis pedis or posterior tibial pulses in either foot. The big toe is slightly bluish and tender to palpation. Which of the following is true about this condition?
(A) It responds well to stenting
(B) A bypass will be curative
(C) Smoking cessation is the cornerstone of management
(D) It is unlikely to be due to arterial occlusion
(E) It most likely represents early atherosclerosis

23. A 65-year-old male carpenter states that his left arm gets tired when he uses it at work, forcing him to stop and rest. In addition, he notes that when using his left arm, he experiences dizziness and vertigo. He has a long-standing history of smoking. Physical examination reveals normal brachial and radial pulses on the right but markedly decreased brachial and radial pulses on the left. In addition, there is an audible bruit just above his left clavicle. Which of the following is true about this condition?

(A) The dizziness and vertigo are due to blood being diverted from the anterior brain circulation
(B) Stenting of the left subclavian artery may be helpful

(C) Systolic blood pressure measurements in right and left arms are likely to be the same

(D) It is most likely due to an inflammatory arteritis

(E) It more commonly affects the right arm

24. A 63-year-old male comes to the physician's office for a nonhealing ulcer on his right great toe and intermittent calf pain. His calf pain is brought on by walking for 10 minutes and relieved by rest. He wakes up at night with pain in his great toe and has to get up to relieve it. He has a past medical history significant for hypertension, hyperlipidemia, and diabetes. He has a 30-packyear smoking history. On physical exam, his temperature is $98.4^{\circ} \mathrm{F}$, pulse is $80 / \mathrm{min}$, blood pressure is $139 / 82$, and respirations are $16 / \mathrm{min}$. His LDL is $70 \mathrm{mg} / \mathrm{dL}$. Exam is significant for absent dorsalis pedis and popliteal pulses on the right. The ulcer appears dull gray without purulence. He has diminished sensation to light palpation in both feet. Ankle-brachial index is 0.3 on the right and 0.7 on the left. What is the most appropriate next step in management?

(A) Diabetic shoe to offload pressure

(B) Formal angiography of the lower extremities

(C) Tight glucose control

(D) Start oral antibiotics

(E) Start heparin

25. A 68-year-old male comes to the physician's office for a nonhealing ulcer in his medial malleolus that has been present for several months. He has a past medical history significant for diabetes and hyperlipidemia. On physical exam, his temperature is $98.9^{\circ} \mathrm{F}$, pulse is $70 / \mathrm{min}$, blood pressure is $133 / 79 \mathrm{mmHg}$, and respirations are $16 / \mathrm{min}$. Physical exam is significant for pitting right leg edema up to the knee. The leg is warm, and the skin is shiny and has a reddish-brown appearance with several enlarged surface veins. The ulcer has granulation tissue without purulence. Dorsalis pedis pulses are 2+ bilaterally. Which of the following is most likely to assist in healing the ulcer?

(A) A compressive dressing impregnated with zinc oxide

(B) Diuretics

(C) A custom-fitted diabetic shoe

(D) Heparin

(E) Oral antibiotics 


\section{Answers}

\section{Answer B}

This patient is presenting with a ruptured abdominal aortic aneurysm (AAA). Hemodynamically unstable patients presenting with classic symptoms and signs of aortic rupture such as hypotension, flank pain, and a pulsatile mass should be taken emergently to the operating room (OR) for immediate control of hemorrhage. Surgical intervention should not be delayed waiting for CT scan of the abdomen (A) or transfusion of packed red blood cells (C). In fact, permissive hypotension is preferable to aggressive fluid resuscitation prior to the OR, as excessive fluids prior to aortic control may lead to more bleeding. In patients with symptomatic non-ruptured or ruptured AAA who are hemodynamically stable, CT scan of the abdomen can be obtained to assess for feasibility of endovascular repair. For patients who are unstable and are not previously known to have AAA and who do not have a palpable pulsatile mass, ultrasound may be performed quickly prior to abdominal laparotomy, to confirm the presence of AAA, but cannot determine rupture (D). Diagnostic peritoneal lavage is not appropriate to rule out a ruptured AAA. The rupture occurs retroperitoneally, so that a DPL would likely be negative (E).

\section{Answer C}

This patient is presenting with acute limb ischemia (ALI) given the pain, pulselessness, and paresthesias (recall the "six Ps"). The etiology of ALI includes thrombus formation (most likely due to long-standing peripheral arterial disease in the legs), embolization (most often from the heart), and trauma. The patient is presenting with an irregularly irregular heart rate, suggesting atrial fibrillation, which is the most common source of arterial embolism. Arterial thrombosis (A) is another cause of ALI. Such patients often have a history of claudication and decreased pulses in the nonischemic leg. Peripheral neuropathy (B) is an important cause of numbness in diabetic patients and would cause decreased sensation in a stocking glove pattern starting on the dorsum of the foot, but would not account for the acute onset and absent pulses. Cerebral ischemia (D) would cause a hemiparesis on the contralateral side and would not cause decreased pulses. Venous thrombosis (E) or DVT would cause warmth, edema, and swelling in the affected leg.

\section{Answer C}

Patients who present with symptoms concerning for stroke should undergo a non-contrast CT scan of the brain to rule out intracranial hemorrhage. Once hemorrhage has been ruled out, consideration should be given for thrombolytic therapy for an ischemic stroke (intravenous if within $3 \mathrm{~h}$ of symptom onset, intra-arterial if within $6 \mathrm{~h}$ of onset). Doppler of the carotid artery (A), echocardiogram (B), ECG (D), or CT angiogram (E) may be performed to evaluate for the cause of the stroke (carotid plaque vs. cardioembolic), but they are not part of the acute management of this patient.

\section{Answer D}

Peripheral arterial disease (PAD) is defined as an anklebrachial index $(\mathrm{ABI})<0.9$. The normal $\mathrm{ABI}$ ranges from 1 to 1.2. Symptomatic PAD can readily be diagnosed via history and physical examination (C), and in fact the classic history, combined with physical exam evidence of diminished pulses, is highly specific, but not sufficiently sensitive. Early PAD can be asymptomatic and is best detected by ABI. Arteriography (E) is invasive and is thus reserved for patients who are to undergo an interventional procedure (E). The pulse exam (B) is very subjective and cannot alone be relied upon to establish a diagnosis of PAD. History of pain in legs (A) with exertion is not specific to PAD.

\section{Answer C}

The United States Preventive Services Task Force (USPSTF) recommends a one-time screening for abdominal aortic aneurysm (AAA) by ultrasonography in men aged 65-75 that have any smoking history. There are no recommendations for women and men who have never smoked. CT of the abdomen (A) is not appropriate for AAA screening. Screening for carotid artery stenosis (B) is not recommended nor screening adults for chronic obstructive pulmonary disease using spirometry (D) or screening for peripheral artery disease with the ankle-brachial index (E).

\section{Answer B}

The most likely etiology is a deep vein thrombosis (DVT) given the swelling and history of colon cancer (hypercoagulability). The best diagnostic test is a venous duplex scan. An ABI (A) is appropriate for suspected peripheral arterial disease (PAD), however, that would not cause swelling. CTA (C) would be a useful follow-up study for PAD prior to a planned intervention. CT of the head (D) would be useful for a suspected stroke. Compartment pressure measurements (E) are sometimes used to rule out compartment syndrome when suspicion is low to moderate; however, it presents with pain with passive motion as well as the other "five Ps": pain, pallor, paresthesias, paralysis, and poikilothermia. It classically presents after trauma or after ischemia reperfusion.

\section{Answer A}

The best indication for CEA is a symptomatic high (70$99 \%$ )-grade ICA stenosis. Symptoms include ipsilateral arm/leg weakness or contralateral amaurosis fugax. Option A fits that description. Since strokes from ICA stenosis are characteristically embolic, once the artery completely occludes (B), there is no longer any embolic risk and thus no benefit from CEA. Patients who have had massive strokes and have no significant neurologic function to preserve (C) 
should not undergo CEA (as the goal is to prevent further damage to the motor cortex). Dizziness and syncope (D) are not symptoms of ICA stenosis. The patient in answer E likely sustained a stroke secondary to his atrial fibrillation, as opposed to carotid artery embolism.

\section{Answer A}

The patient has PAD, manifested by claudication, which is not immediately limb threatening. The diagnosis is confirmed by the $\mathrm{ABI}<0.9$. Further testing is not necessary at this time to confirm the diagnosis. Additional imaging (B-D) should be reserved for patients in whom an intervention is being planned. Initial management of claudication includes smoking cessation, a walking program, and modification of risk factors (lipid-lowering agents, hypertension control). Stenting is a treatment option (after failed medical management), but one must first obtain imaging to properly plan a possible intervention.

\section{Answer D}

The clinical scenario is consistent with a popliteal artery aneurysm. Popliteal artery aneurysms are the most common type of peripheral aneurysms. Risk factors for popliteal aneurysm include smoking, hypertension, male sex, and older age. Patients with a popliteal artery aneurysm have a high risk for aneurysm in the contralateral popliteal artery, femoral arteries, and abdominal aorta. Therefore, patients found to have a popliteal artery aneurysm should be screened with ultrasound for aneurysms at these locations. Hypercoagulable state (B) is associated with deep vein thrombosis. DJD (C) is associated with popliteal (Baker's) cysts. Popliteal aneurysms are not directly associated with thoracic aortic aneurysms (E) or aortic dissection (A).

\section{Answer $\mathrm{E}$}

Chronic venous insufficiency caused by valve incompetence is the most common cause of lower extremity edema. Edema secondary to valve incompetence is worse when the affected extremity is in the dependent position and improves with extremity elevation. Insufficient venous return causes blood to pool in dependent areas and results in increased capillary pressure causing fluid and red cells to leak out of the capillaries. The skin turns reddish brown due to hemosiderin deposition. Peripheral artery disease (A) causes atrophy of the affected muscles, decreased pulses, decreased hair growth, shiny skin, and claudication. Acute DVT (B) causes sudden onset of pain and swelling of the affected limb. Renal protein loss (C), most commonly caused by nephrotic syndrome, leads to decreased oncotic pressure. In the setting of decreased oncotic pressure, there is leakage of fluid into the interstitial space. Nephrotic syndromes would present with hypertension and widespread edema. Reduced cardiac output (D) would present with respiratory symptoms, crackles, and elevated JVP.

\section{Answer E}

Cranial nerve deficits occur in about $8 \%$ of patients after CEA. In 80-90\%, the deficit resolves within 6 months as the injury is due to nerve irritation from retraction and inflammation. In this case, the patient's tongue deviates to the left on protrusion, suggesting an injury to the left $\mathrm{CN}$ XII. Perioperative stroke of the medulla (A) would present with a larger constellation of symptoms. Hematoma (B) is a concern in the immediate postoperative period and would be more likely to compromise the airway than a specific cranial nerve. Injury to the marginal mandibular branch of CN VII (C) would cause a droop at the corner of the mouth. CN X (D) transection would lead to voice hoarseness.

\section{Answer D}

The best medical management for sustained improvement in claudication is a walking program, which can double the walking distance (A). A supervised walking program, consisting of 40-50 min of walking 5 days per week is more effective than a non-supervised program. Contrary to common perception, a walking program does not appear to increase collateral blood flow nor does it reliably increase the ankle-brachial index (B). The exact mechanism by which it improves claudication is not known, but possible mechanisms include improvements in endothelial function, skeletal muscle metabolism, and blood viscosity and a reduction in systemic inflammation. In response to PAD, the distal arterial bed undergoes significant vasodilation. As such, administering pure vasodilators (C) are of no benefit.

\section{Answer A}

This patient is presenting with bowel ischemia secondary to compromised blood flow during open surgical repair of the AAA. During aortic grafting, the inferior mesenteric artery is usually intentionally ligated, which rarely leads to inadequate colonic blood flow. The incidence of colonic ischemia is 7-27\% for open repair of ruptured AAA and 1-13\% for elective AAA repair. $C$. difficile colitis (B) presents with abdominal pain, fever, and watery diarrhea, typically following antibiotic use. Diverticulitis (C) presents with abdominal pain and fever but not bloody diarrhea. Inflammatory bowel disease (D) presents with bloody diarrhea, constipation, fecal incontinence, joint pain, and rash but is more chronic in nature. Colon cancer (E) presents as occult gastrointestinal bleeding, weight loss, and a change in bowel habits.

\section{Answer A}

The most likely etiology of her neurologic deficits is transection of the common peroneal nerve at the fibular head. The most common presentation of peroneal nerve injury at the fibular neck is acute foot drop (difficulty dorsiflexing the foot against resistance or gravity) along with numbness of the dorsum of the foot. Compartment syndrome (B) presents 
with the "five $P s$ ": pain (on passive motion), pallor, paresthesias, paralysis, and poikilothermia following trauma. However, this patient is warm and well perfused and is not complaining of any pain. Arterial thrombosis (C) can occur after trauma; however, the patient has normal distal pulses. Injury to the deep peroneal nerve (D) would cause numbness in the first web space. Pain at the fracture site (E) should not cause such a profound motor deficit nor would it cause a sensory deficit.

\section{Answer B}

Jaw pain with chewing, or jaw claudication, is suggestive of giant cell arteritis, which is managed with steroids. The carotid bruit (A) is a common physical exam finding in patients with carotid stenosis. CT angiogram showing $80 \%$ stenosis (C) confirms the diagnosis of carotid stenosis. Left hand weakness (D) may be found in patients who have had an middle cerebral artery stroke and may or may not be due to carotid emboli. An atrial mass on echocardiogram (E) may be an intracardiac tumor, such as atrial myxoma, that will need to be resected surgically.

\section{Answer A}

Claudication alone, in the absence of ischemic rest pain or tissue loss, is a relatively benign condition that rarely leads to amputation. The risk of amputation is only about 2-5\%. Thus, medical management can safely be utilized as the first step in its management. Five-year mortality in claudicants, however, is significantly increased as compared to non-claudicants. This is predominantly due to an increased risk of myocardial infarction and to a lesser degree stroke.

\section{Answer C}

The patient presents with irreversible acute limb ischemia. Muscle begins to become damaged after $3 \mathrm{~h}$ of ischemia, whereas irreversible ischemia sets in after $6 \mathrm{~h}$. Exam findings that support irreversible ischemia include the 48-h duration of ischemia, profound sensory loss, paralysis of the muscles, mottling of the skin, and inaudible Doppler signals. When acute limb ischemia progresses to irreversible tissue damage, as in the case presented, primary amputation should be performed. Attempting to revascularize an irreversibly ischemic limb (besides not helping the limb) puts the patient at risk of developing reperfusion syndrome, which manifests with severe hyperkalemia, acidosis, and rhabdomyolysis that can lead to renal failure due to acute tubular necrosis.

\section{Answer D}

Although claudication is associated with a very low 5-year risk of limb loss $(2-5 \%)$, it is associated with a significantly reduced 5-year survival (70 \%) as compared to matched controls without claudication. This is primarily due to the associated coronary artery disease and risk of myocardial infarction. Patients with claudication also have an increased risk of carotid stenosis and, as such, an increased risk of stroke, but stroke is not the primary cause of death in these patients.

\section{Answer A}

This patient is suffering from an ischemic stroke. Permissive hypertension is considered beneficial following an ischemic stroke so as to maximize cerebral perfusion pressure (CPP). For ischemic strokes, do not use antihypertensive medications to lower blood pressure unless diastolic is greater than $120 \mathrm{mmHg}$ or systolic is greater than $220 \mathrm{mmHg}$, to ensure proper cerebral perfusion. If it is due to a hemorrhage, keep systolic blood pressure less than $150 \mathrm{mmHg}$. In both cases, avoid nitroglycerin or nitroprusside $(C, D)$, which can serve as potent vasodilators in the cerebral arteries and veins, increasing intracranial pressure (ICP). Recall that increasing ICP can further compromise $\mathrm{CPP}(\mathrm{CPP}=$ mean arterial pressure - ICP). Labetalol can be used safely. In the case of hemorrhage, administer nimodipine (D) to avoid cerebral vasospasms related to irritants in blood. Hydralazine $(\mathrm{E})$ is reserved for pregnant patients.

\section{Answer A}

The triad of Leriche syndrome is (1) buttock and thigh claudication, (2) erectile dysfunction, and (3) absent femoral pulses. It is due to chronic (B) progressive atherosclerosis of the distal aorta and proximal common iliac arteries (not the superficial femoral artery) (C) that eventually occludes the aorta. Since the occlusion is gradual, collaterals have had a chance to enlarge and refill (reconstitute) the femoral arteries distally. Important collaterals come from the superior mesenteric artery, inferior mesenteric artery (superior hemorrhoidal), lumbar arteries, median sacral artery, and internal mammary (via epigastric arteries). Because the aorta and common iliac arteries are occluded, the blood supply to the buttocks (through the internal iliac arteries) is compromised, as is the blood supply to the penis (via the internal pudendal artery, a branch of the internal iliac artery). Thus the patients endorse buttock claudication, as well as erectile dysfunction. Because of the rich collateral supply around the distal aorta and the chronic nature of the occlusion, the ABI typically drops by only $30 \%$ (to about 0.7 ). Thus toe gangrene is rare. Patients with diabetes typically develop atherosclerosis more distally in the leg, in the superficial femoral and tibial arteries (not in the aorta). Acute aortic occlusion is a devastating, severe acute ischemia of both lower legs, which manifests by rapid onset of motor and sensory loss. It is seen following a large embolus from atrial fibrillation lodging at the distal aorta. The sudden onset does not permit adequate collateral enlargement. Untreated, it results in limb loss, massive acidosis, and death. Spinal stenosis (D) compresses nerve roots, leading to generalized weakness of both legs that is worse 
with walking. Unlike claudication, it is usually relieved by leaning forward (such as over a shopping cart).

\section{Answer A}

Warfarin is useful for the prevention of embolic events associated with atrial fibrillation and for the prevention of venous thrombotic events (such as deep venous thrombosis and pulmonary embolus). Since PAD is due to atherosclerosis and not due to embolus/thrombus, there is no evidence that warfarin benefits patients with PAD. Clopidogrel (E) inhibits platelet aggregation by blocking activation of the glycoprotein IIb/IIIa pathway. In patients with PAD, it has been shown to reduce the combined endpoint of stroke, myocardial infarction, and acute limb ischemia. Given its high costs, its routine use in PAD is not recommended. Cilostazol (D) is a quinolinone derivative that inhibits cellular phosphodiesterase. It inhibits platelet aggregation and is a direct arterial dilator. It inhibits vascular smooth muscle proliferation and improves the lipid profile. The exact mechanism by which it improves walking distance in patients with claudication is unclear. Aspirin (B) does not directly improve claudication but is effective in reducing the risks of stroke and acute coronary events, which are common in PAD patients. Statins (C) also does not directly improve walking distance but are beneficial in PAD patients via their lipid-lowering and anti-inflammatory (atherosclerotic plaque stabilizing) properties.

\section{Answer C}

Buerger's disease, also known as thromboangiitis obliterans, is a non-atherosclerotic vascular occlusive disease seen in young $(<40)$, mostly male smokers. It predominantly involves the arteries in the leg below the knee (popliteal and tibial arteries). It also causes venous thrombosis. The cause is unknown. Stenting and surgical bypass (A-B) are ineffective as the occlusions involve the most distal arteries. The only effective treatment is smoking cessation. It is associated with high rates of amputation, especially if the patient continues smoking. The remaining answer choices (D-E) are not used in the management of Buerger's disease.

\section{Answer B}

Subclavian steal syndrome is due to an atherosclerotic stenosis or occlusion (D) of the subclavian artery, most commonly on the left side (E). This leads to claudication symptoms of the affected arm and can be detected on physical examination based on diminished pulses, a significant $(>20 \mathrm{mmHg})$ difference in arm blood pressure (C), and often a bruit above the clavicle. In addition, as the patient exercises, the arteries in the affected arm dilate, lowering resistance, so as to receive more blood. Since the occluded subclavian artery cannot increase blood flow, blood instead travels in a reverse fashion down the vertebral artery (the first branch off the subclavian) down to the arm, essentially stealing blood from the posterior circulation (A), leading to simultaneous symptoms of dizziness and vertigo.

\section{Answer B}

This patient has ischemic rest pain and a nonhealing ulcer, both of which are manifestations of critical peripheral artery disease (PAD) that are considered limb threatening. The patient will likely progress to an amputation unless blood flow is improved. As such, the next step is to obtain arterial imaging of the lower extremities. This can be done by either CT angiography, MR angiography, or formal transfemoral arteriography in anticipation of either balloon angioplasty, stenting, or an arterial bypass. Tight glucose control (C) will not help in achieving healing of an ischemic ulcer. Diabetic shoes are useful for ulcers that form over bony prominences in the setting of neuropathy. Although the patient has evidence of neuropathy, the location of the ulcer (distal toe), its appearance (lack of granulation tissue), absent pulse, and low ABI indicate that the ulcer is from arterial insufficiency. Oral antibiotics (D) are not indicated as there is no evidence of infection. Heparin (E) is helpful for acute limb ischemia but not for chronic PAD.

\section{Answer A}

The patient presents with a classic case of a venous stasis ulcer. These ulcers develop as a result of chronic venous insufficiency, due to vein valve incompetence. Such incompetence can be inherited or be the result of a prior DVT that scars and damages valves, rendering them incompetent. The ensuing venous hypertension results in increased capillary pressure causing fluid and red and white blood cells to leak out of the capillaries. When the red cells break and lyse, they release the iron-containing hemosiderin and lead to the classic reddishbrown discoloration seen in stasis dermatitis. The pooling of blood leads to capillary damage and activation of an inflammatory process. The exact cause of ulceration in venous stasis is unclear, but is likely a combination of leukocyte activation, endothelial damage, and intracellular edema. The cornerstone of treatment of venous stasis ulcers is compression therapy (A). The Unna boot is a compressive gauze that contains zinc oxide and calamine to promote wound healing. Diuretics (B) do not benefit venous insufficiency. Diabetic shoes (C) would be appropriate for a neuropathic ulcer, which would typically be located over a bony prominence. Heparin (D) would be indicated for a DVT but not for chronic venous stasis. Venous stasis ulcers can become infected and require antibiotics (E), however, their routine use is not recommended. 